\title{
Permanent Closure of MFC Biodiesel Underground Storage Tank 99ANL00013
}

October 2012

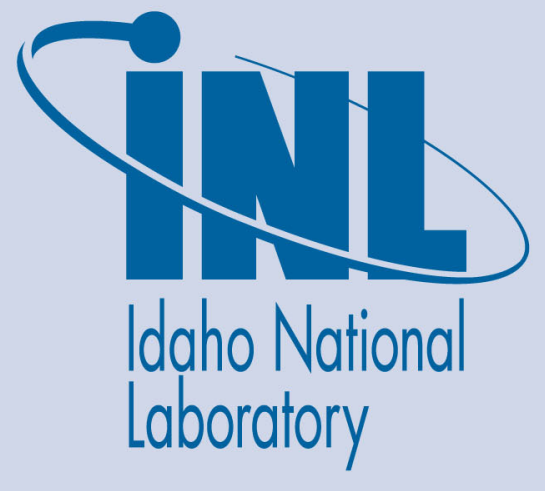

The INL is a U.S. Department of Energy National Laboratory operated by Battelle Energy Alliance 


\section{DISCLAIMER}

This information was prepared as an account of work sponsored by an agency of the U.S. Government. Neither the U.S. Government nor any agency thereof, nor any of their employees, makes any warranty, expressed or implied, or assumes any legal liability or responsibility for the accuracy, completeness, or usefulness, of any information, apparatus, product, or process disclosed, or represents that its use would not infringe privately owned rights. References herein to any specific commercial product, process, or service by trade name, trade mark, manufacturer, or otherwise, does not necessarily constitute or imply its endorsement, recommendation, or favoring by the U.S. Government or any agency thereof. The views and opinions of authors expressed herein do not necessarily state or reflect those of the U.S. Government or any agency thereof. 


\section{Permanent Closure of MFC Biodiesel Underground Storage Tank 99ANL00013}

October 2012

Idaho National Laboratory Idaho Falls, Idaho 83415

http://www.inl.gov

Prepared for the

U.S. Department of Energy

Office of Nuclear Energy

Under DOE Idaho Operations Office

Contract DE-AC07-05ID14517 


\section{CONTENTS}

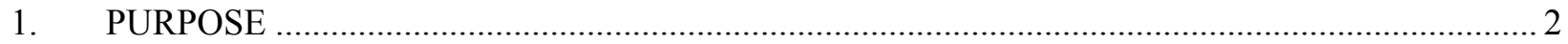

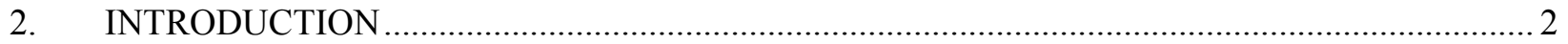

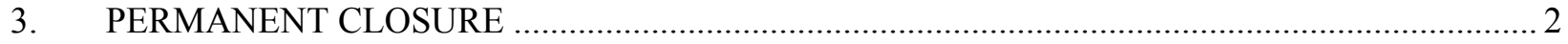

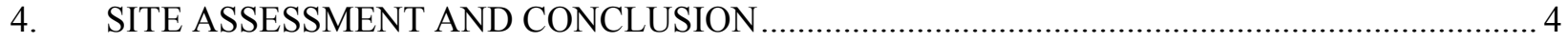

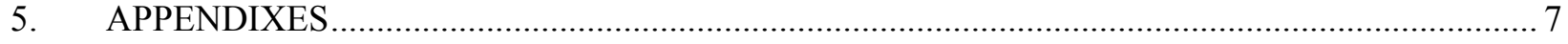

Appendix A, Notification of Closure (CCN 227777)............................................................. 8

Appendix B, Sampling and Analysis Plan Correspondence (CCN 228161) ................................ 16

Appendix C, Notification of Underground Storage Tank Removal Correspondence (CCN

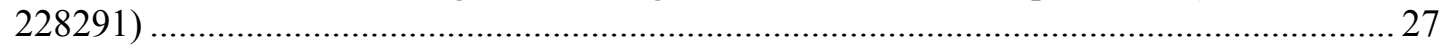

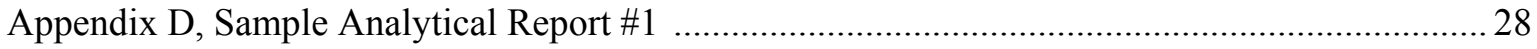

Appendix E, DEQ Notification of Sample Results for MFC UST Closure (CCN 228521)........... 99

Appendix F, Sample Analytical Report \#2 ....................................................................... 100

Appendix G, Notification of Underground Storage Tank Soil Samples \#2 (CCN 228585)........... 149

Appendix H, Discussion and Verbal Approval Regarding the Use of EPA Region 9

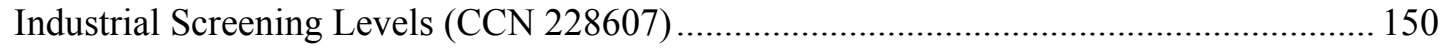

Appendix I, Spreadsheet Comparison of EPA Region 9 Industrial Screening Levels vs.

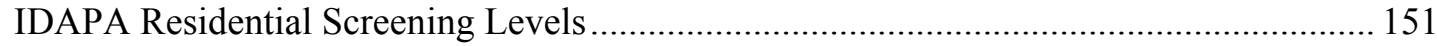

Appendix J, E-mail to DEQ with Soil Analytical Analysis \#1 and \#2 and Spreadsheet

Comparison of EPA Region 9 Industrial Screening Levels vs. IDAPA Residential

Screening Levels

\section{FIGURES}

Figure 1. MFC Biodiesel Underground Storage Tank Removal........................................................... 5

Figure 2 . Tank Rendered Unusable (holes punched in both sides) ..................................................... 6

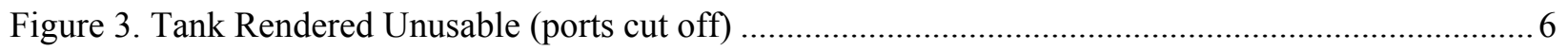

Figure 4. MFC Biodiesel Underground Storage Tank after Cleaning .................................................. 7

\section{TABLES}

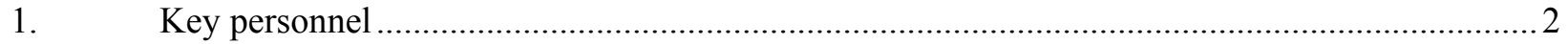




\section{Permanent Closure of MFC Biodiesel Underground Storage Tank}

\section{PURPOSE}

This closure package documents the site assessment and permanent closure of the Materials and Fuels Complex biodiesel underground storage tank 99ANL00013 in accordance with the regulatory requirements established in 40 CFR 280.71, "Technical Standards and Corrective Action Requirements for Owners and Operators of Underground Storage Tanks: Out-of-Service UST Systems and Closure."

\section{INTRODUCTION}

The MFC biodiesel underground storage tank 99ANL00013 is a 4,000-gallon, single-wall, asphalt coated steel tank with cathodic protection and safe suction above ground steel piping, and is located at the Idaho National Laboratory (INL) Materials and Fuels Complex (MFC) facility. The tank is owned by the Department of Energy and operated by Battelle Energy Alliance (BEA). The tank was installed in 1980. The tank originally held gasoline for motor vehicle use and was later converted to diesel, then biodiesel for bus use via a fuel dispenser. This tank is identified under Facility ID 6-120614 as tank number 99ANL00013 in the Idaho Department of Environmental Quality (DEQ) underground storage tank database. The tank leak detection system was a Veeder-Root TLS-350 tank monitoring system with automatic tank gauging.

An Idaho Department of Environmental Quality (DEQ) Underground Storage Tank (UST) inspection in September 2011 identified two violations to this tank. Violation \#1: failure to install a properly designed cathodic protection system for a metal tank (informal warning) and violation \#2: failure to ensure proper operation of a cathodic protection within six (6) months of installation and every three (3) years thereafter (formal warning). As a result of these violations, BEA decided to remove this tank and replace it with an aboveground storage tank (AST) with aboveground piping. Key personnel that were involved in this closure/demolition activity are listed in Table 1.

Table 1. Key personnel.

\begin{tabular}{lll}
\hline \multicolumn{1}{c}{ Title/Organization } & \multicolumn{1}{c}{ Name } & \multicolumn{1}{c}{ Responsibilities } \\
\hline Project Manager & Mark Cole & Project execution and completion \\
Facility Manager & Scott Lyman & Manage/approve facility activities \\
Environmental Compliance & Kerry Nisson & Coordinate UST closure activity \\
Project Supervisor & Lucien Frederick & Project implementation \\
\hline
\end{tabular}

In preparation for demolition and permanent tank closure, the remaining fuel in the line and tank was pumped out on September 3, 2012, and the tank was removed on September 11, 2012 and permanently closed in accordance with 40 CFR 280.71.

\section{PERMANENT CLOSURE}

In accordance with 40 CFR 280.71(a), a 30-day closure notification was mailed on July 9, 2012, (Appendix A, CCN 227777) notifying Idaho DEQ of BEA's intent to permanently close MFC tank 99ANL00013 biodiesel underground storage tank. A sampling and analysis plan was developed for sampling the soils around and under the underground storage tank system. The Idaho DEQ Regional Office in Idaho Falls (i.e., Steve Heaton) requested a copy of the sampling and analysis plan in preparation for the closure. 
On August 8, 2012, the sampling and analysis plan was e-mailed to the Idaho DEQ Regional Office (Appendix B, CCN 288161).

On August 9, 2012, an e-mail response from Idaho DEQ was received from Steve Heaton stating the sampling and analysis plan for pending UST work at MFC had been reviewed and the e-mail would serve as the Department's endorsement to execute the plan.

On August 27, 2012 a call was placed to Steve Heaton at the Idaho DEQ Regional Office informing him of BEA's intent to begin removal of the UST and to identify if DEQ wanted be present during any part of the removal process. Steve stated that DEQ did not plan to visit the INL during the UST removal and to keep him informed as the project progressed. It was stated that DEQ would be notified if there were any evidence of tank leakage during the removal (Appendix C - CCN 228291).

On September 10, 2012, INL's Environmental Monitoring personnel collected soil samples around and under the underground storage tank and supply line in accordance with the sampling and analysis plan. Soil samples were sent to ALS Environmental Fort Collins, Colorado for analysis. Laboratory analysis was requested for chemicals of interest for gasoline and diesel found in Tables 1 and 2 of IDAPA 58.01.24.800.

On September 19, 2012, the ALS Environmental sent the soil sample analytical report (Appendix D) to INL's Environmental Monitoring personnel. The analytical report identified semi-volatile levels of Benzo(A)Anthracene (Ground water Protection via petroleum contaminants in soil leaching to ground water), Benzo(B)Fluoranthene (direct contact), and Benzo(A)Pyrene (direct contact) above the residential screening levels for chemicals of interest identified in table 2, of IDAPA 58.01.24.800.

On September 20, 2012, a call was placed to notify the DEQ Regional Office (24 hour notification) of the sampling analysis results that the chemicals Benzo(A)Anthracene, Benzo(B)Fluoranthene, and Benzo(A)Pyrene exceeded the IDAPA residential screening levels (Appendix E - CCN 228521). As an option, DEQ suggested removing more soil and resampling, as this may bring the levels into the desired screening level range. DEQ agreed that this phone call would serve as the 24 -hour notification.

On September 20, 2012, INL's Environmental Monitoring personnel collected a second set of soil samples including samples from the soil pile staged from the tank removal. Soil samples were sent to ALS Environmental Fort Collins, Colorado for analysis. The same analysis was requested.

On September 25, 2012, ALS Environmental sent the soil sample analytical report, to INL's Environmental Monitoring personnel (Appendix F). The analytical report only identified levels of Benzo(A)Pyrene above the screening levels identified in table 2 of IDAPA 58.01.24.800.

On September 26, the Idaho DEQ Regional Office was notified (Appendix G - CCN 228585) that sampling analytical analysis results still showed that Benzo(A)Pyrene exceeded the IDAPA residential screening levels. DEQ suggested that the Idaho National Laboratory (INL) use commercial screening levels, as the INL was not, nor ever would be residential. Commercial screening levels could not be found in the Idaho Risk Evaluation Manual for Petroleum Releases. A search for commercial screening levels revealed Region 9, Regional Screening Levels for Industrial Soil Supporting screening levels on the Environmental Protection Agency website. Both sets of analytical analysis results were compared to the Region 9 Industrial Soil Supporting table using ingestion for the target risk, as this was the most conservative value. The Benzo(A)Anthracene, Benzo(B)Fluoranthene, and Benzo(A)Pyrene were below the industrial screening levels. The INL developed a spreadsheet showing the comparison of EPA Region 9 Industrial Screening Levels vs. IDAPA Residential Screening Levels (Appendix H). 
On September 27 (note - the e-mail attachment states September 26, which is a typographical error), a call was placed to the DEQ Regional Office explaining the actions taken above (Appendix $\mathrm{H}$ - CCN 228607). DEQ responded that if there was no guidance found for commercial screening levels in the Idaho Risk Evaluation Manual for Petroleum Releases, then it would be acceptable to use the EPA Region 9 guidance and gave verbal authorization to continue. DEQ requested that the EPA Region 9 information be included in this tank closure plan. An e-mail was sent to DEQ with the sampling analysis and the INL developed spreadsheet (Appendix I) for review. A request was made to contact the INL if any issues were encountered when reviewing the spreadsheet.

Construction personnel accessed the inside of the tank through hole in both sides of the tank and removed all remaining liquids (i.e., a small amount at the slightly sloped end). The inside of the tank was mopped out with absorbent pads. Waste that was generated during this process was disposed of through INL's Waste Generator Services personnel. All liquids and accumulated sludges were removed, meeting the requirement in 40 CFR 280.71(b), "Permanent Closure and Changes-In-Service" (Figure 4).

\section{SITE ASSESSMENT AND CONCLUSION}

This site assessment was performed in accordance with IDAPA 58.01.24.200, "Risk Evaluation Process." A screening evaluation was performed according to the previously submitted sampling and analysis plan of the MFC biodiesel fuel tank (Appendix B). This included collection of media-specific (soil) samples and analysis for the chemicals of interest (benzene, toluene, xylenes, MTBE, and naphthalene) for gasoline, diesel, and biodiesel (IDAPA 58.01.24.200.a and b. and IDAPA 58.01.24.800.01, Table 1.)

Per 40 CFR 280.71"Permanent Closure and Changes-In-Service" All liquids and accumulated sludges were removed from the UST.

Sample results (Appendix E and G) were received on September 19 and September 25, 2012, respectively, and were compared to the maximum media-specific (soil) petroleum contaminant concentrations identified in IDAPA 58.01.24.800.02, Table 2. Both sample results showed semi-volatile detection for the chemicals of interest above the levels identified in Table 2.

The Idaho DEQ Regional Office advised the INL to look at commercial screening levels in the Idaho Risk Evaluation Manual for Petroleum Releases, as the site at MFC was not a residential site. The MFC biodiesel tank removal site is not near ground or surface water.

A search for commercial screening levels revealed Region 9, Regional Screening Levels for Industrial Soil Supporting screening levels on the Environmental Protection Agency website (http://www.epa.gov/region9/superfund/prg/).

Both sets of analytical sample results were compared to the Region 9 Industrial Soil Supporting table using the most conservative target risk value - ingestion. All chemicals of concern were below than the industrial screening levels for ingestion, found on the Region 9 Industrial Soil Supporting table.

A spreadsheet (Appendix I) was developed showing the sample analysis results with comparison between IDAPA 58.01.24.800.02, Table 2 Residential Use Screening levels and the Region 9, Regional Screening Level, Industrial Soil Supporting table, Screening Levels for Chemical Contaminants. The comparison shows the semi-volatiles (Benzo(A)Anthracene, Benzo(B)Fluoranthene, and Benzo(A)Pyrene) that were above the IDAPA Residential Use Screening levels were below the Region 9, Industrial Soil Supporting table, Screening Levels for Chemical Contaminants.

The Idaho DEQ Regional Office was called on September 26, 2012 explaining the actions taken, as mentioned above. DEQ responded that if there was no guidance found for commercial screening levels in the Idaho Risk Evaluation Manual for Petroleum Releases, then it would be acceptable to use the EPA Region 9 guidance and gave verbal authorization to continue. DEQ requested that the EPA Region 9 
information be included in this tank closure plan. An e-mail was sent to DEQ with the sample analysis and the spreadsheet utilized for review. A request was made to DEQ to contact the INL if any issues were encountered when reviewing the spreadsheet.

Based on the methods used above and with the Idaho DEQ Regional Office verbal acceptance with using Region 9, industrial soil screening levels, the INL is petitioning for approval of site closure of the Materials and Fuels Complex biodiesel underground storage tank 99ANL00013. A final updated copy of the 30-day closure notification has been included.

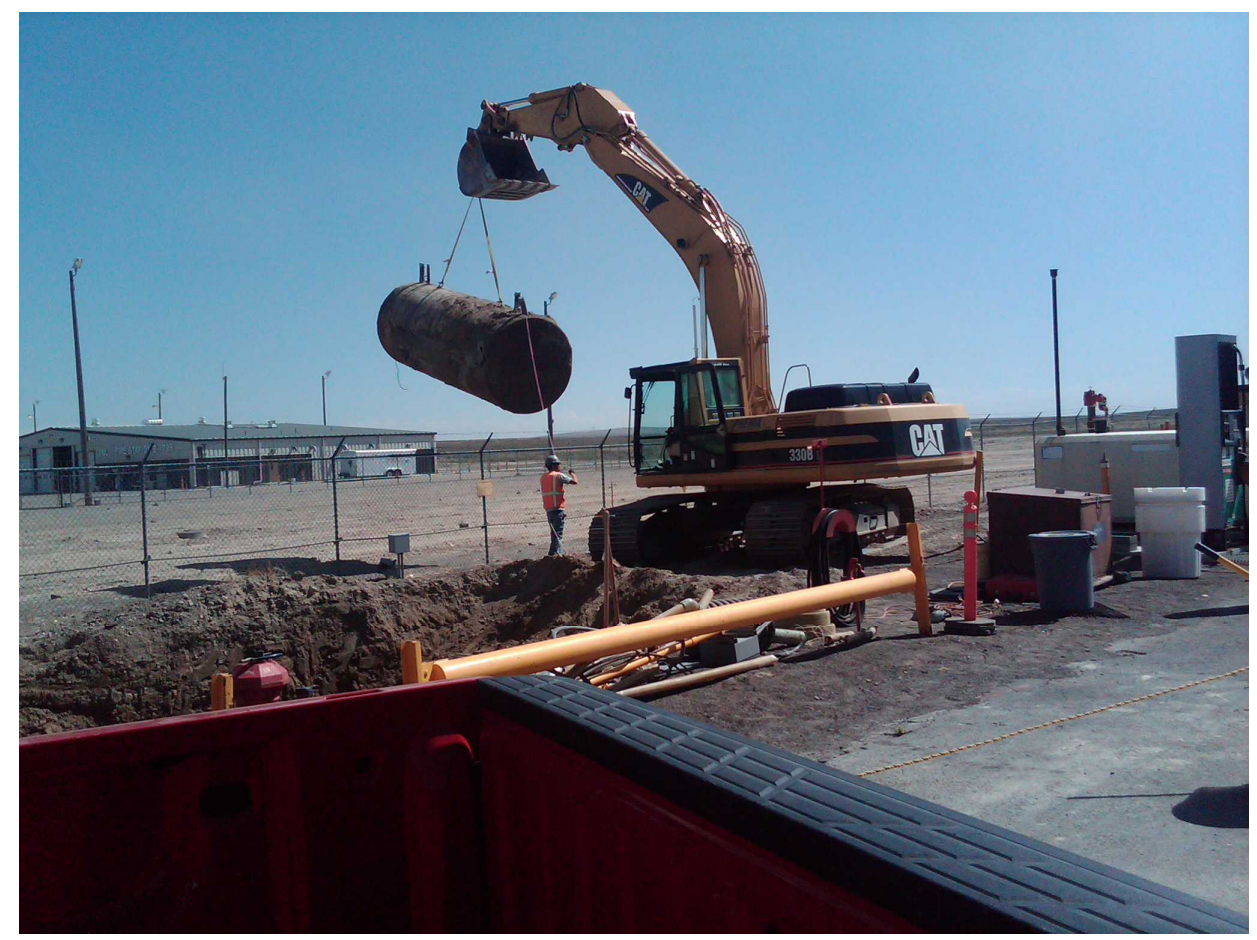

Figure 1. MFC Biodiesel Underground Storage Tank Removal. 


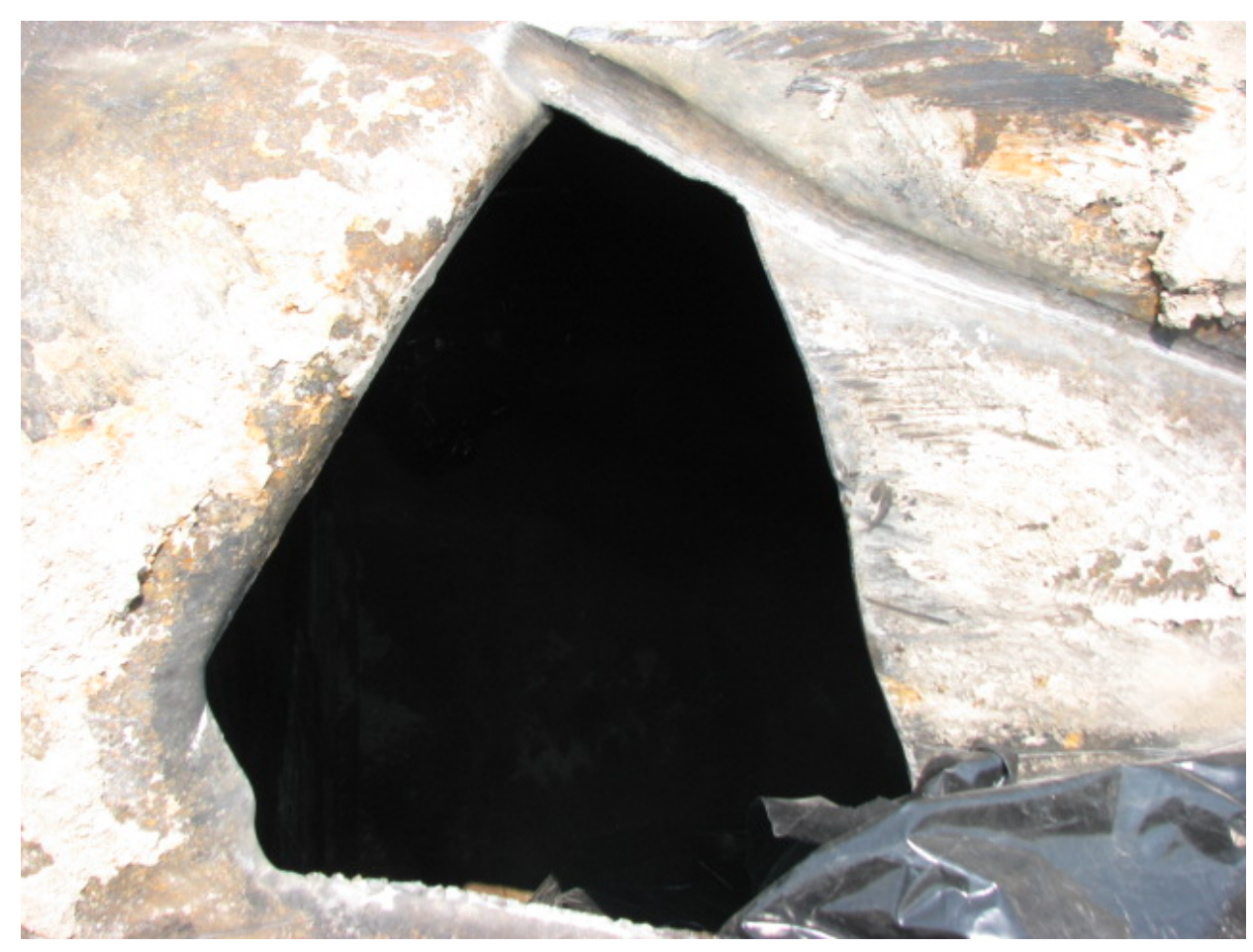

Figure 2. Tank Rendered Unusable (holes punched in both sides)

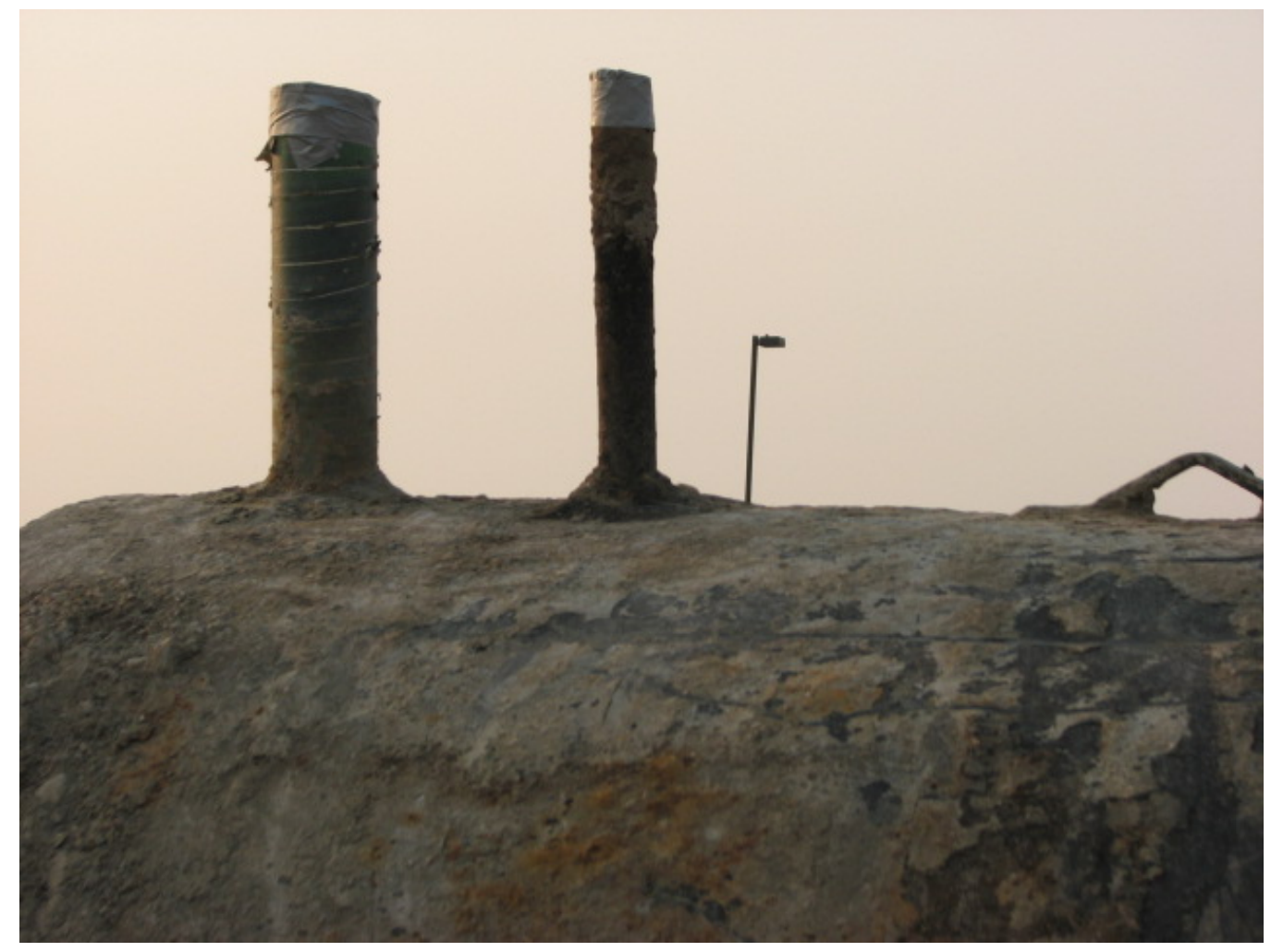

Figure 3. Tank Rendered Unusable (ports cut off) 


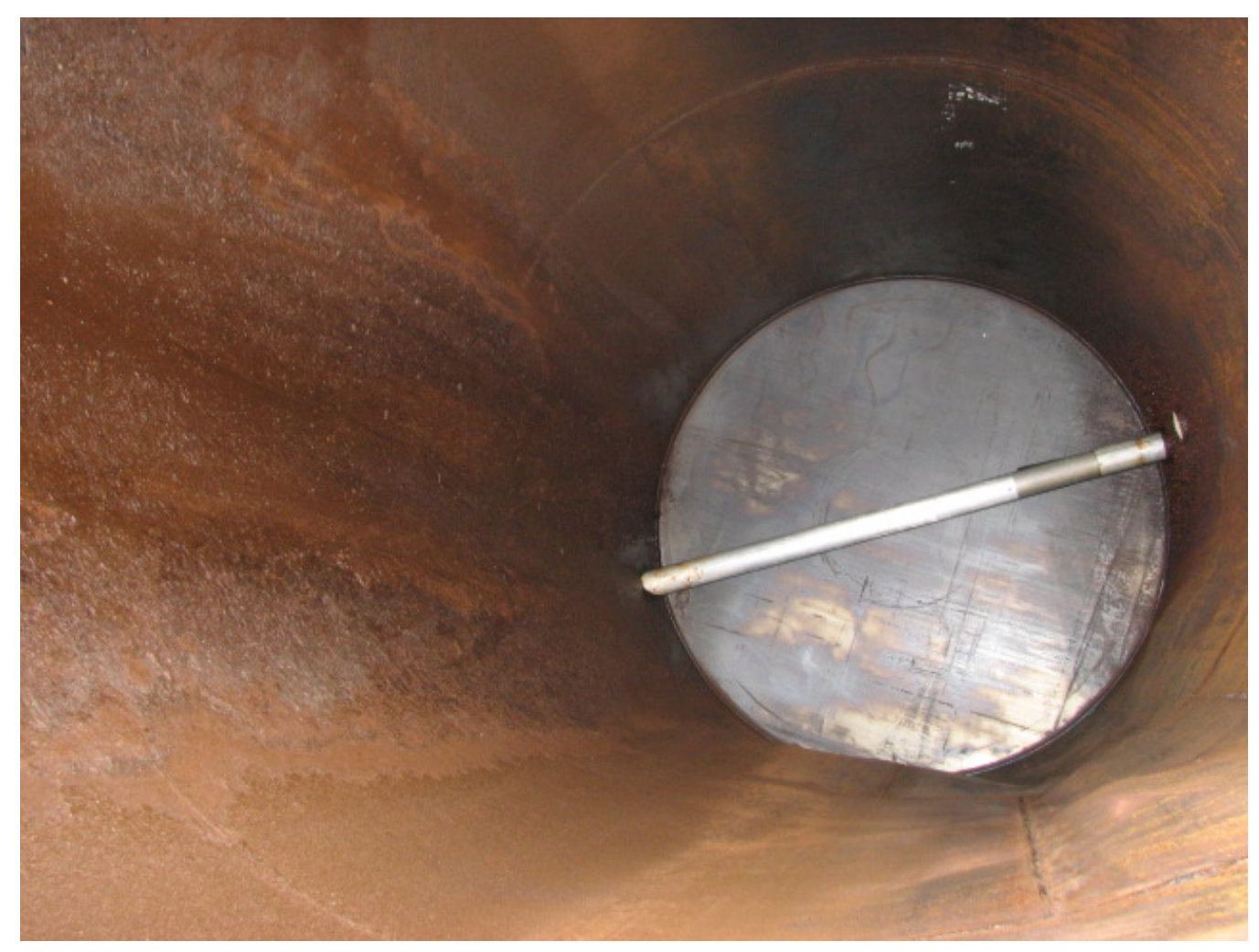

Figure 4. MFC Biodiesel Underground Storage Tank after Cleaning

\section{APPENDIXES}

Appendix A, Notification of Closure (CCN 227777)

Appendix B, Sampling and Analysis Plan Correspondence (CCN 228161)

Appendix C, Notification of Underground Storage Tank Removal Correspondence (CCN 228291)

Appendix D, Sample Analytical Report \#1

Appendix E, DEQ Notification of Sample Results for MFC UST Closure (CCN 228521)

Appendix F, Sample Analytical Report \#2

Appendix G, Notification of Underground Storage Tank Soil Samples \#2 (CCN 228585)

Appendix H, Discussion and Verbal Approval Regarding the Use of EPA Region 9 Industrial Screening Levels (CCN 228607)

Appendix I, Spreadsheet Comparison of EPA Region 9 Industrial Screening Levels vs. IDAPA Residential Screening Levels

Appendix J, E-mail to DEQ with Soil Analytical Analysis \#1/\#2 and Spreadsheet Comparison of EPA Region 9 Industrial Screening Levels vs. IDAPA Residential Screening Levels 


\section{Appendix A \\ Notification of Closure (CCN 227777)}

July 9,2012

CCN 227777

Kristi Lowder

UST Coordinator

Idaho Department of Environmental Quality

1410 North Hilton

Boise, ID 83706

SUBJECT: Notification for Underground Storage Tank Closure at Idaho National Laboratory, Materials and Fuel Complex

Dear Ms. Lowder:

This letter provides 30-day notification of intent to close an underground storage tank located at the Idaho National Laboratory, Materials and Fuels Complex (Site ID Number 7230/Facility ID Number 6-120614/ Tank ID Number 99ANL00013). This notice is submitted for Battelle Energy Alliance, LLC (BEA) as the operating contractor for this project.

The enclosed provides the necessary information and notification required by Title 40 CFR 280, Subpart G. Please note that a follow up notification will be submitted with the required site assessment and the information required in Section XI. BEA has communicated with the Idaho DEQ Regional Office in Idaho Falls (S. Heaton and S. Short) regarding this closure and will provide them with a sample plan and a 48-hour notification prior to closure.

If you have any questions, please contact Kerry Nisson (208) 533-7102.

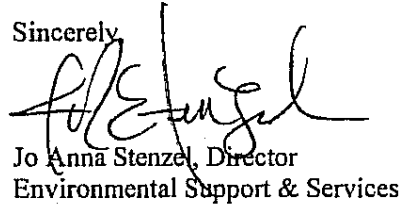

KLN:AT

Enclosure
cc: J. Alvarez, DN, MS 3695
R. M. Kauffman, DOE-ID, MS 1216
P. K. Bowers, DOE-1D, MS 1226
C. D. Melbihess, INL, MS 3406
R. R. Chase, INL, MS 3695
S. M. Olson, DOE-ID, MS 1240
S. D. Dossett, INL, MS 3405
T. L. Perkins, DOE-ID, MS 1216
R. A. Gallegos, DOE-ID, MS 1216
S. Short, DEQ, Idaho Falls
J. J. Grossenbacher, INL, MS 3695
D. M. Storms, INL, MS 3898
S. Heaton, DEQ, Idaho Falls
J. R. Sturm, DOE-ID, MS 1216

P.O. Box 1625 • 2525 North Fremont Ave. Idaho Falls, Idaho 83415 • 208-526-0111 www.inl.gov Battelle Energy Alliance, LLC 
Ms. Kristi Lowder

July 9, 2012

CCN 227777

Page 2

bcc: P. J. Brcidenbach, MS 6146

A. E. Carvo, MS 6134 AC

T. L. Carlson, MS 3405 Tic portelecor

L. M. Coe-Leavitt, MS 6134 ef per telecar

J.F. Graham, MS 3428 HeG bur telecar

B. K. Griffith, MS 7113

S. D. Lee, MS 3405

S. L. Lyman, MS 6172

C. D. Melbihess, MS 3406

T. A. Miller, MS 3428

R. V. Nelson, MS 3406

R. A. Nickelson, MS 3406

K. L. Nisson, MS 6134 \<

Environmental Correspondence, MS 3405, email: ENVAFF@inl.gov

INL Correspondence Control, MS 3640, email: BEACC@inl.gov

J. A. Stenzel Letter Log (JAS-62-12)

Uniform File Code: 61043

Disposition Authority: ENV3-d-1

Retention Schedule: Cut off after certificate of closure is received. Destroy 10 years after cut off.

NOTE: Original disposition authority, retention schedule, and Uniform Filing Code applied by the sender may not be appropriate for all recipients. Make adjustments as needed. 


\begin{tabular}{|l|l|}
\hline NOTIFICATION FOR UNDERGROUND STORAGEITANK SYSTEMS & Facility ID 6-120614 \\
\hline Idaho Department of Environmental Quality, 1410 N Hilton, Boise ID 83706 & \\
\hline
\end{tabular}

\section{TYPE OF NOTIFICATION}

区Notice $\square$ New Facility (site diagram \& install testing docs required) $\square$ Updates $\bigotimes$ Closure
INSTRUCTIONS - See additional instructions on page 7

Please type or use lnk. This form must be completed for each location containing underground storage tanks. If more than five (5) tanks are owned at this location, photocopy the following sheets, and staple continuation sheets to the form (pages 3,4 , $5,86)$

\section{CENERALINORMATION}

Notification is required by law for all underground tanks that have been used to store regulated substances since January 1,1974, that are in the ground

as of May 8, 1986, or that are brought into use after May 8, 1986. The

information requested is required by Section 9002 of the Resource

Conservation and Recovery Act, (RCRA), as amended.

The primary purpose of this notification program is to locate and evaluate

underground tanks that will store, do store, or have stored petroleum or hazardous substances. It is expected that the informatton you provide will be based on reasonably avaliable records, or in the absence of such records, your knowledge, belief, or recollection.

Who must notify? Unless exempted, owners of underground tank systems that store or will store regulated substances must notify DEQ.

1. Owner means -

a) In the case of an underground storage tank in use on November 8,1984 , or brought into use ffter that date, any person who owns an underground storage tank used for the storage, use, or dispensing of regulated substances, and b) in the case of any underground storage tank in use before November 8 1984, but no longer in use on that date, any person who owned such tank immediately before the discontinuation of its use

c) in the case of a new installation on or after April 2, 2008, any person who wil install an underground storage tank system

d) in the case of an underground storage lank closure, any person who will remove or close in place such tank

e) any facllity that has undergone any changes to faclity information or lank system status (only amended tank information needs to be included).

What tanks are included? Underground storage tank is defined as any one or combination of tanks that (1) is used to contain an accumulation of "regulated substances," and (2) whose volume (Including connected underground piping) is $10 \%$ or more beneath the ground. Some examples are underground tanks storing gasoline, used oll, diesel fuel, industrial solvents, pesticides, hericides, or fumigants.

What tanks are excluded? Tanks with a capacity of 110 gallons or tess are not subject to nolification. Other tanks excluded from notificallon are:

1. fam or residential tanks of 1,100 gallons or less capacity used for storing motor fuel for noncommercial purposes;

2. tanks used for storing heating oll for consumptive use on the premises where stored;

3. septic tanks

4. pipeline facilties (Including gathering lines) regulated under the Natural Gas Pipeline Safety Act of 1968, or the Hazardous Liquld Plpeline Safety Act of

1978 , or which is an intrastate plpellne faclity regulated under State laws:

5. surface impoundmenls, pits, ponds, or lagoons:

6. storm water or waste water collection systems;

7. flow-through prooess tanks:

8. liquid traps or associated gathering lines directly related to oll or gas production and gathering operalions:

9. storage tanks situated in an underground area (such as a basement, cellar, mine working drift, shaft, or tunnel) if the storage tank is situated upon or above the surface of the floor.

What substances are covered? The notification requirements apply to underground storage tanks that contain regulated substances. This includes any substance defined as hazardous in section 101 (14) of the Comprehensive Environmenlal Response, Compensation and Liability Act of 1980 (CERCLA), with the exception of those substances regulated as hazardous waste under Subtitle C of RCRA. It also includes petroleum, e.g., crude oll or any fracton thereof which is liquild at standard conditions of temperature and pressure $(60$ degrees Fahrenheit and 14.7 pounds per square inch absolute).

Where to notify? Send completed forms to:

\section{UST Coordinator}

Idaho Department of Environmental Quality 1410 N. Hilton

Boise, ID 83706 Telephone: (208) 373-0502

When to notify? Owners of underground storage tank systems that are still in the ground must notfy immedialely. Owners who bring underground storage tanks into use after May 8,1986 , must notity within 30 days of bringing the tanks into use. Owners who will install an underground storage tank system must notify 30 days prior to the installation. Owners who will replace $100 \%$ of piping connected to a single underground storage tank must notify 24 hours prior to the replacement. Owners who will close an underground storage tank must notify 30 days prior to the closure. Owners who have closed an underground storage tank must notify and indlcate the date of closure.

Penalties: Any owner who knowingly falls to notify or submits false information shall be subject to a clvil penalty.

EPA estimates public reporting burden for this form to average 30 minutes per response including time for reviewing instructions,

gathering and maintaining the data needed and completing and reviewing the form. Send comments regarding thls burden estimate to Chief, Information Policy Brand PM-223, US Environmental Protection Agency, $401 \mathrm{M}$ Street, Washington D.C. 20460, marked "Attention Desk Officer for EPA." This form amends the previous notification Desk Officer for EPA." This form amends the pre
form as printed in 40 CFR Part 280 . Appendix.. .

$$
\text { I. OWNERSHIP OF TANK(S) }
$$

Name U.S. Department of Energy, Idaho Operations Office (DOE-ID)

Mailing Address 1955 Freemont Avenue

City Idaho Falls

State Idaho

ZIP Code 83401

County Bonneville

Phone Number (With Area Code) (208) 526-2493

Email sturmir@id.doe.gov

\section{LOCATION OF TANK(S)}

(If same as Section I, mark box here $\square$ ) Name Materials and Fuels Complex - Idaho National Laboratory

Street Address (no PO Box)

City Scoville

State Idaho

ZIP Code 83415

County Bingham 


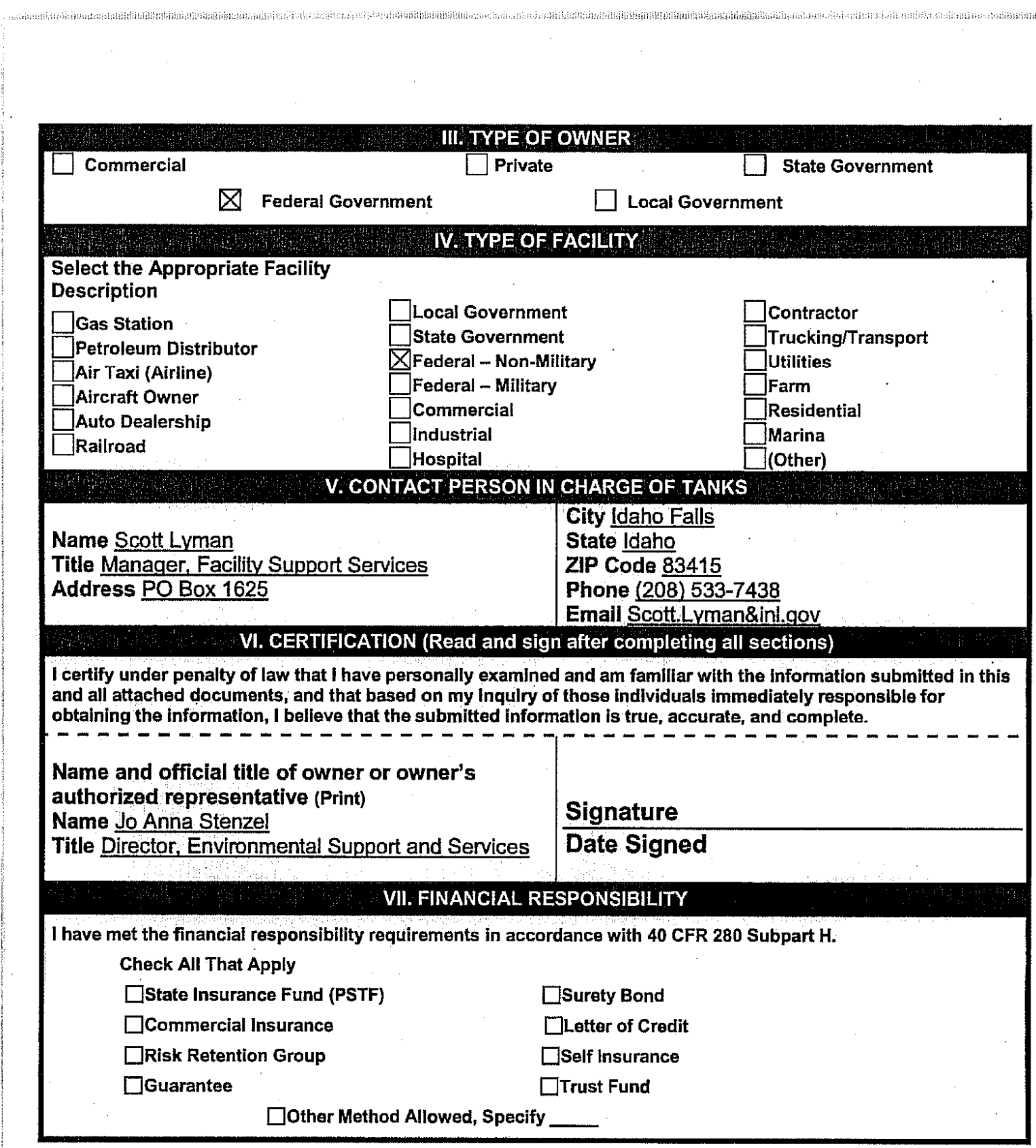




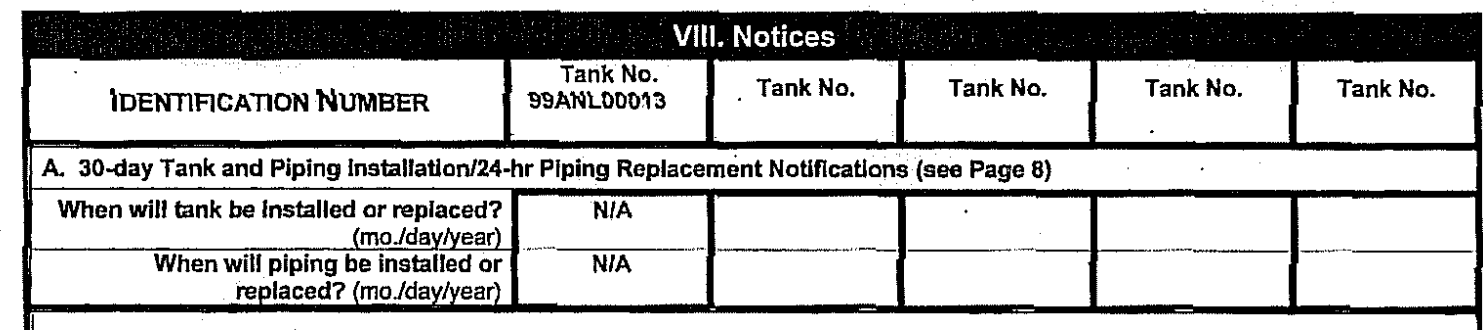

B. 30-day Notice of Closures (see Page 8)

\begin{tabular}{|c|c|c|c|c|}
\hline When will tank be closed? (mo./day/year) & $\sim 8 / 15 / 12$ & & \\
\hline Date tank was last used? (mo./day/year) & $-8 / 6 / 12$ & & \\
\hline
\end{tabular}

Closure to be performed by:

Company Battelle Energy Alliance Site Supervisor: Lucien Frederick

Phos: (2001) o3337805.

\section{Ground Water Protection Measures}

(Check the applicable box)

The underground storage tank system IS within 1000' of a drinking water source or system. $\bigotimes$

The underground storage tank system IS NOT within w000' of a drining water source or system.

If the owner and installer certify that the underground storage tank system is not within 1000' of an axlsting publle water system or potable drinking water well, the owner or operator must provide and maintain documentation showing that a reasonable investigation of water systems and drinking.water wells was undertaken.

\begin{tabular}{|c|c|c|c|c|c|}
\hline IDENTIFICATION NUMBER & $\begin{array}{l}\text { Tank No: } \\
\text { g9ANLDo013 }\end{array}$ & Tank No. & Tank No. & Tank No. & Tank No. \\
\hline Compartmentalized/Manifolded Tanks? & Select & Select & Select & Stalect & Select \\
\hline Emergency Generator Tank? & $\mathrm{No}$ & Selact & Solect & Select & Select \\
\hline
\end{tabular}

A. Status of Tank

\begin{tabular}{|c|c|c|c|c|c|}
\hline Currently in Use & Yes & Select & Select & Select & Select \\
\hline $\begin{array}{r}\text { Temporarily Out of Use } \\
\text { (Complate Section XI, Estimated Data Last Used) }\end{array}$ & $\ldots$ & & & & \\
\hline $\begin{array}{l}\text { Permanently Out of Use } \\
\text { (Complete Section Xl, tanks removed or closed in place) }\end{array}$ & Select & Select & Select & Select & Select \\
\hline Date of Installation (mo./day/year) & 1980 & & & & \\
\hline Total Capacity (gallons) & 4000 & & & & \\
\hline
\end{tabular}

B. Material of Tank Construction (Mark all that apply)

Fiberglass Reinforced Plastic

Cathodically Protected Steel (STIP-3)

Cathodically Protected Steel (Impressed

Current)

Epoxy Coated Stee

Composite (Steel with Fiberglass)

Asphalt Coated or Bare Stee

Double Walled

Lined Interior

Polyethylene Tank Jacket

Excavation Liner

Unknown

\begin{tabular}{|c|c|c|c|c|}
\hline$\square$ & $\square$ & $\square$ & $\square$ & $\square$ \\
\hline$\square$ & $\square$ & $\square$ & $\square$ & $\square$ \\
\hline$\square$ & $\square$ & $\square$ & $\square$ & $\square$ \\
\hline$\square$ & $\square$ & $\square$ & $\square$ & $\square$ \\
\hline$\square$ & $\square$ & $\square$ & $\square$ & $\square$ \\
\hline$\square$ & $\square$ & $\square$ & $\square$ & $\square$ \\
\hline$\square$ & $\square$ & $\square$ & $\square$ & $\square$ \\
\hline$\square$ & $\square$ & $\square$ & $\square$ & $\square$ \\
\hline$\square$ & $\square$ & $\square$ & $\square$ & $\square$ \\
\hline$\square$ & $\square$ & $\square$ & $\square$ & $\square$ \\
\hline$\square$ & $\square$ & $\square$ & $\square$ & $\square$ \\
\hline
\end{tabular}

Page 3 


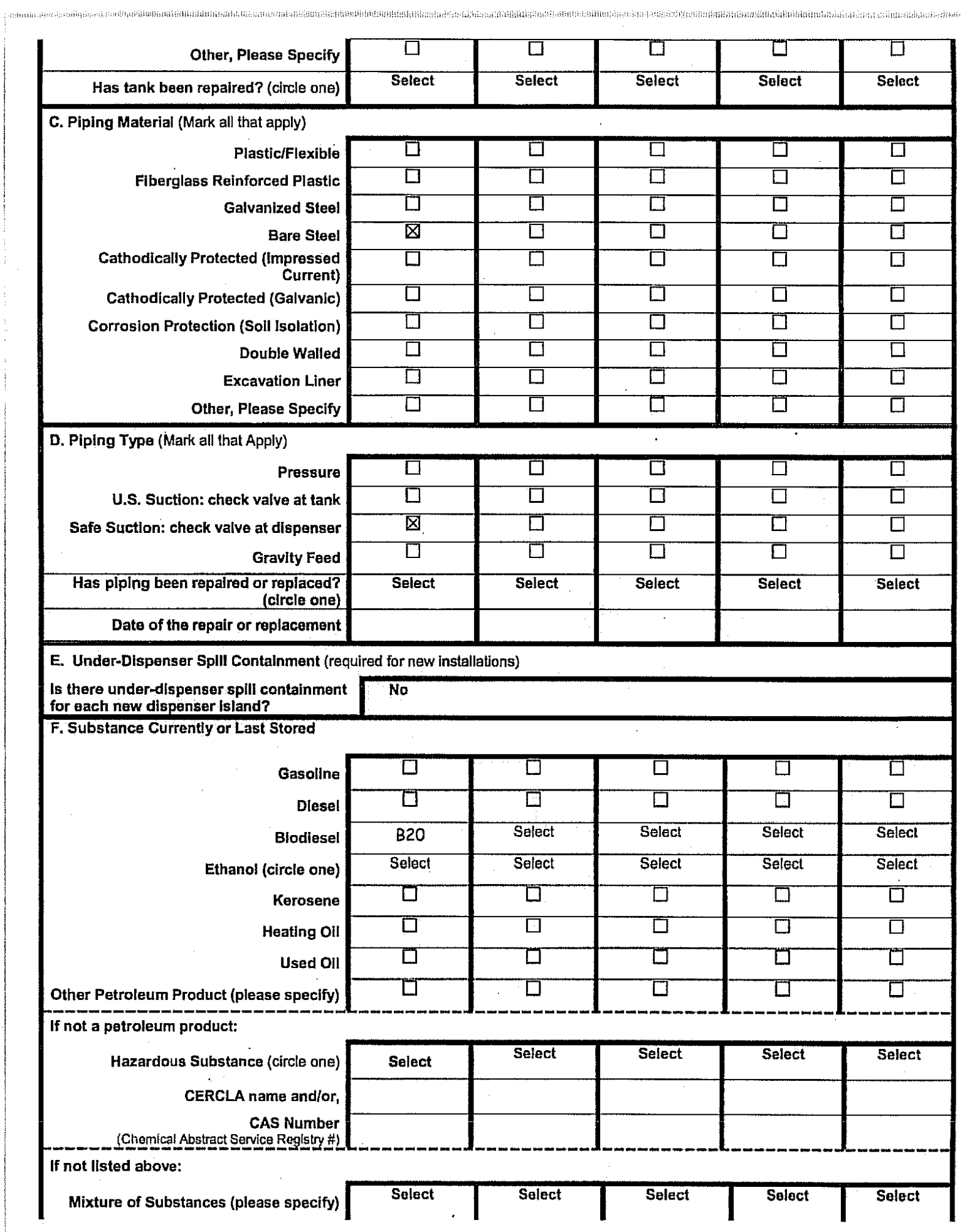

Page 4 


\begin{tabular}{|c|c|c|c|c|c|}
\hline TANK IDENTIFICATION NUMBER & $\begin{array}{l}\text { Tank No. } \\
\text { goANLODO13 }\end{array}$ & Tank No & Tank No. & Tank No & Tank No. \\
\hline \multicolumn{6}{|l|}{ Closing of Tank } \\
\hline Tank was removed from ground & $\square$ & $\square$ & $\square$ & $\square$ & $\bar{\square}$ \\
\hline Tanks was closed In ground & $\square$ & $\square$ & $\square$ & $\square$ & प] \\
\hline \multicolumn{6}{|l|}{$\begin{array}{r}\text { Estimated date last used } \\
\text { (mo./day/year) } \\
\end{array}$} \\
\hline \multicolumn{6}{|l|}{$\begin{array}{r}\text { Date tank closed } \\
\text { (mo./day/year) } \\
\end{array}$} \\
\hline \multicolumn{6}{|l|}{$\begin{array}{r}\text { Tank Fllled with inert material } \\
\text { (indicate material) }\end{array}$} \\
\hline $\begin{array}{r}\text { Change in Service (no longer holds a } \\
\text { regulated substance) }\end{array}$ & $\square$ & $\square$ & $\square$ & $\square$ & {[} \\
\hline $\begin{array}{l}\text { Site Assessment Completed and } \\
\text { submitted to DEQ (circle one) }\end{array}$ & Select & Select & Select & Select & Select \\
\hline Evidence of a leak detected (circle one) & Select & Select & Select & Select & Select \\
\hline Release reported to DEQ & Select & Select & Select & Select & Select \\
\hline Date release reported to DEQ & & & & & \\
\hline
\end{tabular}

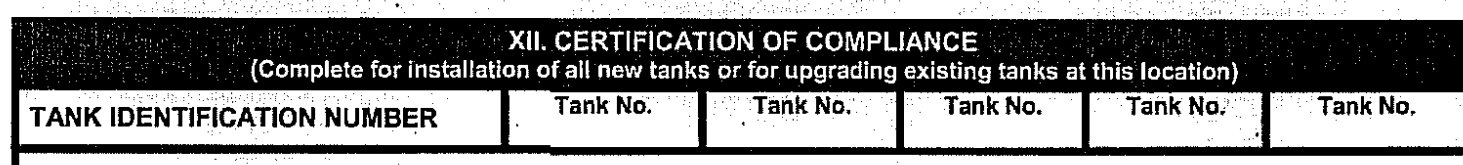

A. Installation (Mark all that apply)

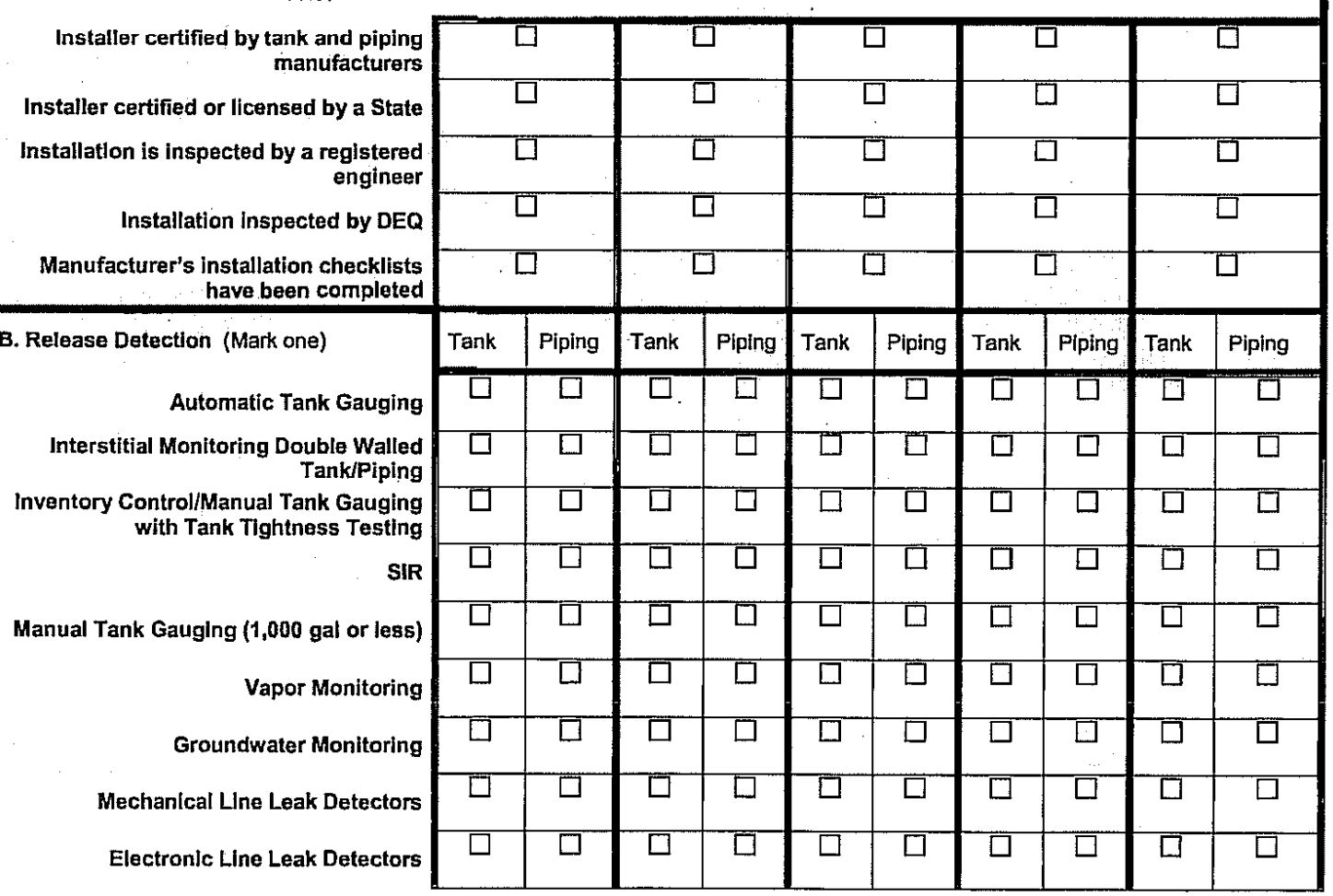

Page 5 


\begin{tabular}{l}
$\begin{array}{l}\text { Annual Line Tightness Testing } \\
\text { 3-year Line Tightness Testing }\end{array}$ \\
\cline { 2 - 7 }
\end{tabular}

Page 6 


\section{Appendix B \\ Sampling and Analysis Plan Correspondence (CCN 228161)}

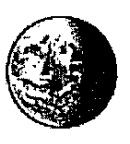

$<$ Steven.Heaton@deq.idaho.

gov>

08/09/2012 12:54 PM
To <kerry.nisson@inl.gov>, <Stacy.Short@deq.idaho.gov>

cc

bcc

Subject Re: CCN: 228161 PLN-4244-Sampling and Analysis Plan for Post Removal Soil Sampling at MFC Biodiesel Underground Storage Tank

History: \& This message has been replied to

Thanks Kerry.

I have reviewed your attached Sampling and Analysis Plan for pending UST work at MFC. Please let this email serve as the Department's endorsement to execute the plan at your convenience.

Steve

Sent from my Verizon Wireless Device

Kerry L Nisson wrote:

I inadvertently listed the CCN 288161 in the subject line, which is incorrect. The correct CCN is 228161.

Kerry L. Nisson

Nuclear Operations Environmental Support - UST TPOC

Office (208) 533-7102

Cell (208) 569-4721

email: kerry.nisson@inl.gov

Mail Stop 6134

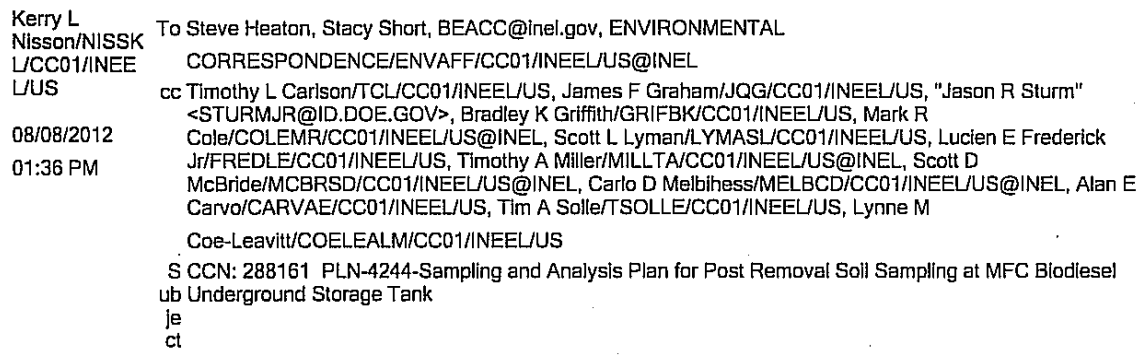

Steve/Stacy

Attached is a copy of PLN-4244-Sampling and Analysis Plan for Post Removal Soil Sampling at MFC Biodiesel Underground Storage Tank. This is the sampling plan that will be used during the removal of the Materials and Fuels Complex biodiesel tank. 
Please review and respond back with any comments you may have. If you do not have any comments, please let me know.

If you have any questions, please contact me for resolution. My phone numbers are listed below.

Thanks

Kerry L. Nisson

Nuclear Operations Environmental Support - UST TPOC

Office (208) 533-7102

Cell (208) 569-4721

email: kerry.nisson@inl.gov

Mail Stop 6134 

Soil Sampling at MFC Biodiesel Underground Storage Tank

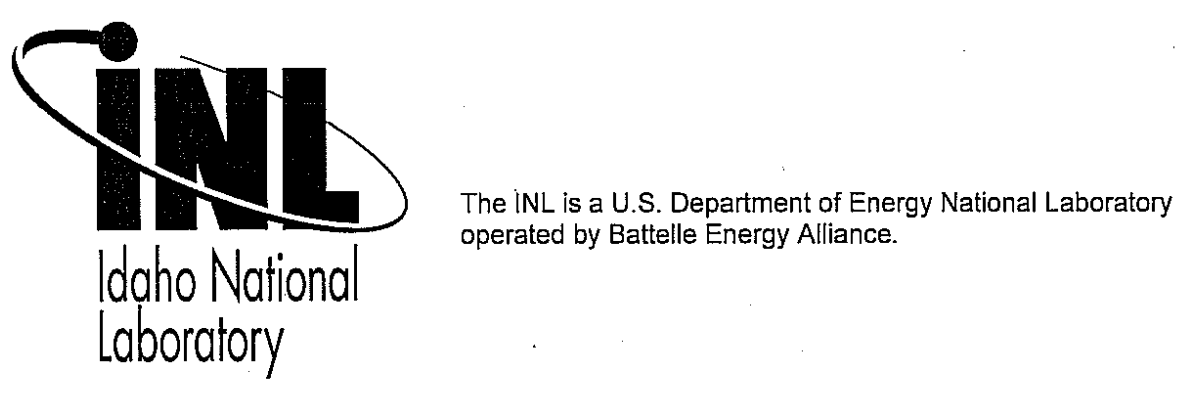




\begin{tabular}{|c|c|c|c|}
\hline SAMPLING AND ANALYSIS PLAN FOR & Identifier: & PLN-4244 & \\
\hline POST-REMOVAL SOLL SAMPLING AT & Revision: & 0 & \\
\hline $\begin{array}{l}\text { MFC BIODIESEL UNDERGROUND } \\
\text { STORAGE TANK }\end{array}$ & Effective Date: & DRAFT & Page: iii of iv \\
\hline
\end{tabular}

\begin{tabular}{|l|l|l|l|}
\hline MFC Biodicsel Tank Removal & Plan & & eCR Number: 605284 \\
\hline
\end{tabular}

\section{CONTENTS}

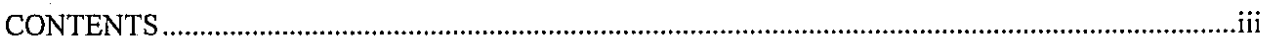

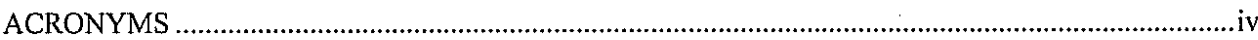

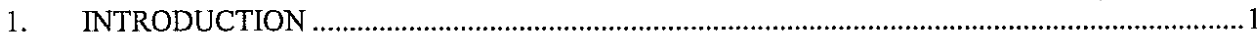

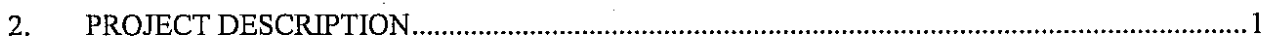

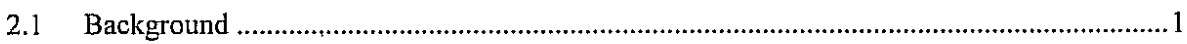

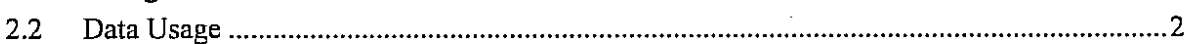

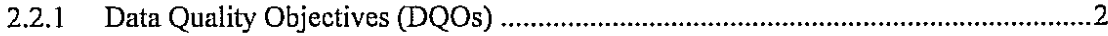

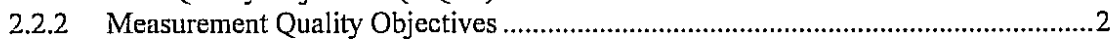

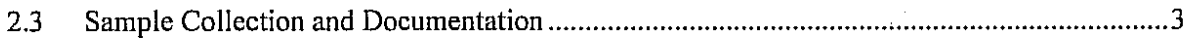

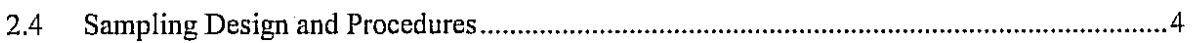

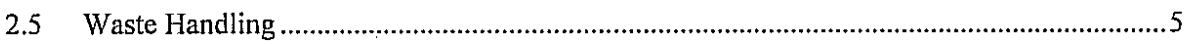

3. DATA QUALITY REQUIREMENTS AND ASSESSMENTS …................................................

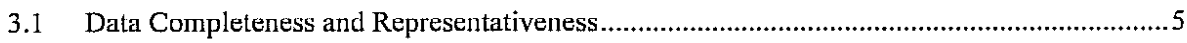

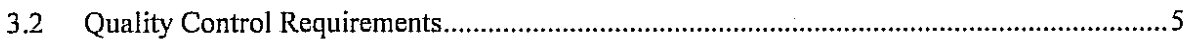

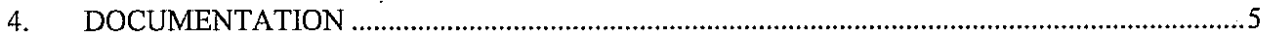

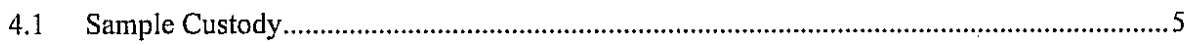

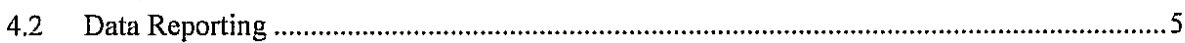

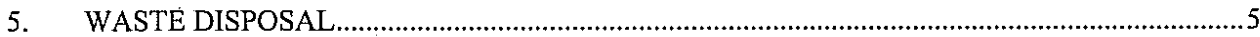

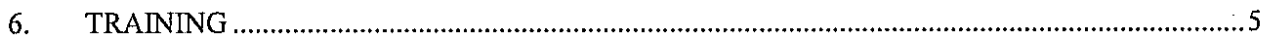

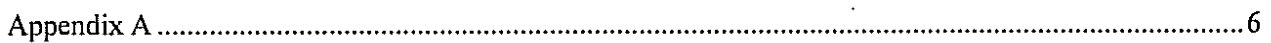

\section{FIGURES}

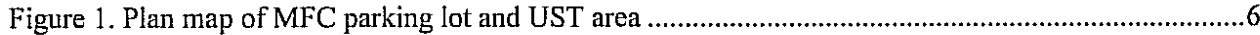

\section{TABLES}

Table 1. Residential use screening levels (IDAPA 58.01.24) ....................................................................

Table 2. Soil sample locations, bottle requirements, analysis, and preservation .......................................... 


Idaho National Laboratory
\begin{tabular}{|c|lll|}
\hline SAMPLING AND ANALYSIS PLAN FOR & Identifier: & PLN-4244 & \\
POST-REMOVAL SOIL SAMPLING AT & Revision: & 0 & \\
MFC BIODIESEL UNDERGROUND & Effective Date: & DRAFT & Page: iv of iv \\
STORAGE TANK & & & \\
\hline
\end{tabular}

\section{ACRONYMS}

$\begin{array}{ll}\text { AA } & \text { Alternative Actions } \\ \text { BEA } & \text { Battelle Energy Alliance } \\ \text { CFR } & \begin{array}{l}\text { Code of Federal Regulations } \\ \text { chain of custody }\end{array} \\ \text { DQO } & \text { data quality objectives } \\ \text { GDE } & \text { Guide } \\ \text { IDAPA } & \text { Idaho Administrative Procedures Act } \\ \text { IH } & \text { industrial hygienist } \\ \text { INL } & \text { Idaho National Laboratory } \\ \text { LI } & \text { Laboratory Instructions } \\ \text { MCP } & \text { management control procedure } \\ \text { MFC } & \text { Materials and Fuels Complex } \\ \text { MQO } & \text { Measurement Quality Objective } \\ \text { MS/MSD } & \text { matrix spike/matrix spike duplicate } \\ \text { QA } & \text { quality assurance } \\ \text { QC } & \text { quality control } \\ \text { RCRA } & \text { Resource Conservation Recovery Act } \\ \text { RCT } & \text { radiological control technician } \\ \text { SAP } & \text { Sampling and Analysis Plan } \\ \text { TCLP } & \text { Toxicity Characteristic Leaching Procedure } \\ \text { UTS } & \begin{array}{l}\text { underground storage tank } \\ \text { UTS }\end{array} \\ \text { Wniversal Treatment Standards (from RCRA land disposal regulations) } \\ \text { Waste Generator Services }\end{array}$


Idaho National Laboratory

\begin{tabular}{|c|lll|}
\hline Idaho National Laboratory & & \\
SAMPLING AND ANALYSIS PLAN FOR & Identifier: & PLN-4244 \\
POST-REMOVAL SOIL SAMPLING AT & Revision: & 0 & \\
MFC BIODIESEL UNDERGROUND & Effective Date: & DRAFT & Page: 1 of 6 \\
STORAGE TANK & & & \\
\hline
\end{tabular}

\section{INTRODUCTION}

This plan was prepared in response to a request from Kerry Nisson for confirmatory soil sampling and analysis after permanent removal of MFC biodiesel underground storage tank (UTS) 99ANL00013. After the tank and any visibly-contaminated soil is removed, soil sampling will be performed to confirm that the soil meets the Table 2 Residential Use Screening Levels in IDAPA 58.01.24, Application of Risk Based Corrective Action at Petroleum Release Sites.

Tank 99ANL00013 is a 4000-gal underground storage tank containing biodiesel (the location is shown in Figure 1 in Appendix A). The tank probably held leaded gas in the 1980s and is known to have held unleaded gasoline in the 1980s and 1990s. The tank was converted from gasoline to diesel in 1992 and to biodiesel in November 2009. The tank is used by site personnel to fill government vehicles.

Samples will be collected using hand tools such as augers, scoops, and spoons.

The residential use screening levels for the soil after removal of Tank \#99ANL00013 are listed in Table 1.

Table 1. Residential use screening levels (IDAPA 58.01.24).

\begin{tabular}{|l|c|}
\hline \multicolumn{1}{|c|}{ Compound } & Screening Level (mg/kg) \\
\hline Benzene & 0.025 \\
\hline Toluene & 6.6 \\
\hline Ethylbenzene & 0.25 \\
\hline Total Xvlenes & 27 \\
\hline Naphthalene & 0.12 \\
\hline Methyl tert-butyl ether (MTBE) & 0.08 \\
\hline Ethylene Dibromide (EDB) & 0.0001 \\
\hline 1,2-Dichloroethane & 0.013 \\
\hline Acenaphthene & 200 \\
\hline Anthracene & 3200 \\
\hline Benz(a)anthracene & 0.09 \\
\hline Benzo(a)pvrene & 0.02 \\
\hline Benzo(b)fluoranthene & 0.2 \\
\hline Benzo(k)fluoranthene & 1.9 \\
\hline Chrvsene & 9.5 \\
\hline Fluoranthene & 1400 \\
\hline Fluorene & 240 \\
\hline Pyrene & 1000 \\
\hline
\end{tabular}

\section{PROJECT DESCRIPTION}

\subsection{Background}

This sampling effort must be able to show the remaining soil is in compliance with $40 \mathrm{CFR}$ Sec. 280.72 "Assessing the Site at Closure or Change-In-Service," and Table 1\&2 of IDAPA 58.01.24.800.02, "Residential Use Screening Levels." Namely, sampling and analysis must measure for the presence of a release where contamination is most likely to be present at the UST site. 
Idaho National Laboratory

\begin{tabular}{|c|c|c|c|}
\hline SAMPLING AND ANALYSIS PLAN FOR & Identifier: & PLN-4244 & \\
\hline POST-REMOVAL SOIL & Revision: & 0 & \\
\hline $\begin{array}{c}\text { MFC BIODIESEL UNDERGROUND } \\
\text { STORAGE TANK }\end{array}$ & Effective Date: & DRAFT & 2 of 6 \\
\hline
\end{tabular}

\subsection{Data Usage}

The analytical results from the samples will be used to verify the remaining soils are within screening levels for residential use as defined in IDAPA 58.01.24.800.02.

\subsubsection{Data Quality Objectives (DQOs)}

The objective of this sampling activity is to obtain representative samples of soil from locations where contamination is most likely to be present at the UST site after its removal. The data quality objectives (DQO) process specifies objectives for collecting the data. Each of the following subsections corresponds to a step in the DQO process, and the output for each step is provided as appropriate.

\subsubsection{Decision Statement}

The objective of this soil characterization activity is to answer the following principal study question: Are the concentrations of the constituents of concern in the soil within the screening levels in Table 2 of IDAPA 58.01.24.800.02?

The alternative actions to be taken based on resolution of the principal study question are:

1. If the concentrations do not exceed the residential use screening levels, a petition for site closure will be submitted.

2. If concentrations exceed residential use screening levels then other options will be needed for corrective action.

Combining the principal study question and alternative actions results in the following decision statement: Determine whether the soil in question meets the screening levels for residential use and petition for site closure; or it needs corrective action. If free product is observed in the excavation, it will be removed prior to confirmation sampling.

\subsubsection{Decision Inputs}

The decision inputs come from the laboratory results from the collected samples.

\subsubsection{Study Boundaries}

The study boundary for this Sampling and Analysis Plan (SAP) is the soil in the excavation after removal of the UST.

\subsubsection{Decision Rule}

If concentrations in the soil do not exceed residential use screening levels in IDAPA 58.01.24.800.02 Table 2, then a petition for site closure will be submitted. If outside the ranges in IDAPA 58.01.24.800.02 Table 2, then corrective action will be required.

\subsubsection{Measurement Quality Objectives}

Measurement Quality Objectives (MQOs) are specifications for precision, accuracy, and completeness that measurements must meet to produce acceptable data.

The laboratory evaluates the accuracy of the analyses with an internal laboratory QA/QC program utilizing matrix spikes. The accuracy goals established by the laboratory will be considered acceptable for this project. The completeness goal for this project is $100 \%$ which means all samples will be collected and all analysis will be acceptable and contain no rejected data.

The required sample bottles, preservation, and holding times are listed in Table 2, "Soil sample locations, bottle requirements, analysis, and preservation." 


\begin{tabular}{|c|lll|}
\hline $\begin{array}{c}\text { SAMPLING AND ANALYSIS PLAN FOR } \\
\text { POST-REMOVAL SOIL SAMPLING AT } \\
\text { MFC BIODIESEL UNDERGROUND } \\
\text { STORAGE TANK }\end{array}$ & Revision: & PLN-4244 & \\
Effective Date: & DRAFT & Page: 3 of 6 \\
\hline
\end{tabular}

\subsection{Sample Collection and Documentation}

Collection of the samples will be conducted using the work control process. Specifically, LI-328, "Idaho National Laboratory Miscellaneous Media Umbrella Sampling," will be used to identify the hazards and mitigations, training, and PPE. In the case of finding something unexpected while sampling (e.g., free product), personnel will institute "a stop work." LI-328 identifies key personnel who assess the hazards (e.g., $\mathrm{IH}$ ). Collection of the samples, decontamination, shipment, labeling, and chain of custody, will follow MC.P, LI, and/or guides (GDFs) as identified helnw.

- LWP-9101, "INL Procedure Usage"

- GDE-9103, "Conduct of Operations Guidance for Communications"

- MCP-8523, "Managing Hazardous and Nonhazardous Samples"

- LI-359, "Cleaning of Environmental Monitoring Services Sampling Equipment"

- LI-355, "Working in Environmental Monitoring Services Sample Preparation Areas (SPA)"

- LI-328, "Idaho National Laboratory Miscellaneous Media Umbrella Sampling."

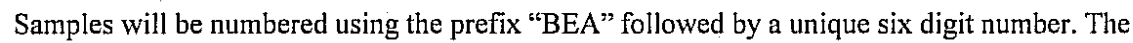
sample numbers, labels and Chain of Custody (COC) forms will be generated in the SAP Application program by Environmental Site Services personnel. Field activities for this sampling event will be documented in the non-routine logbook INL-NR-007. 


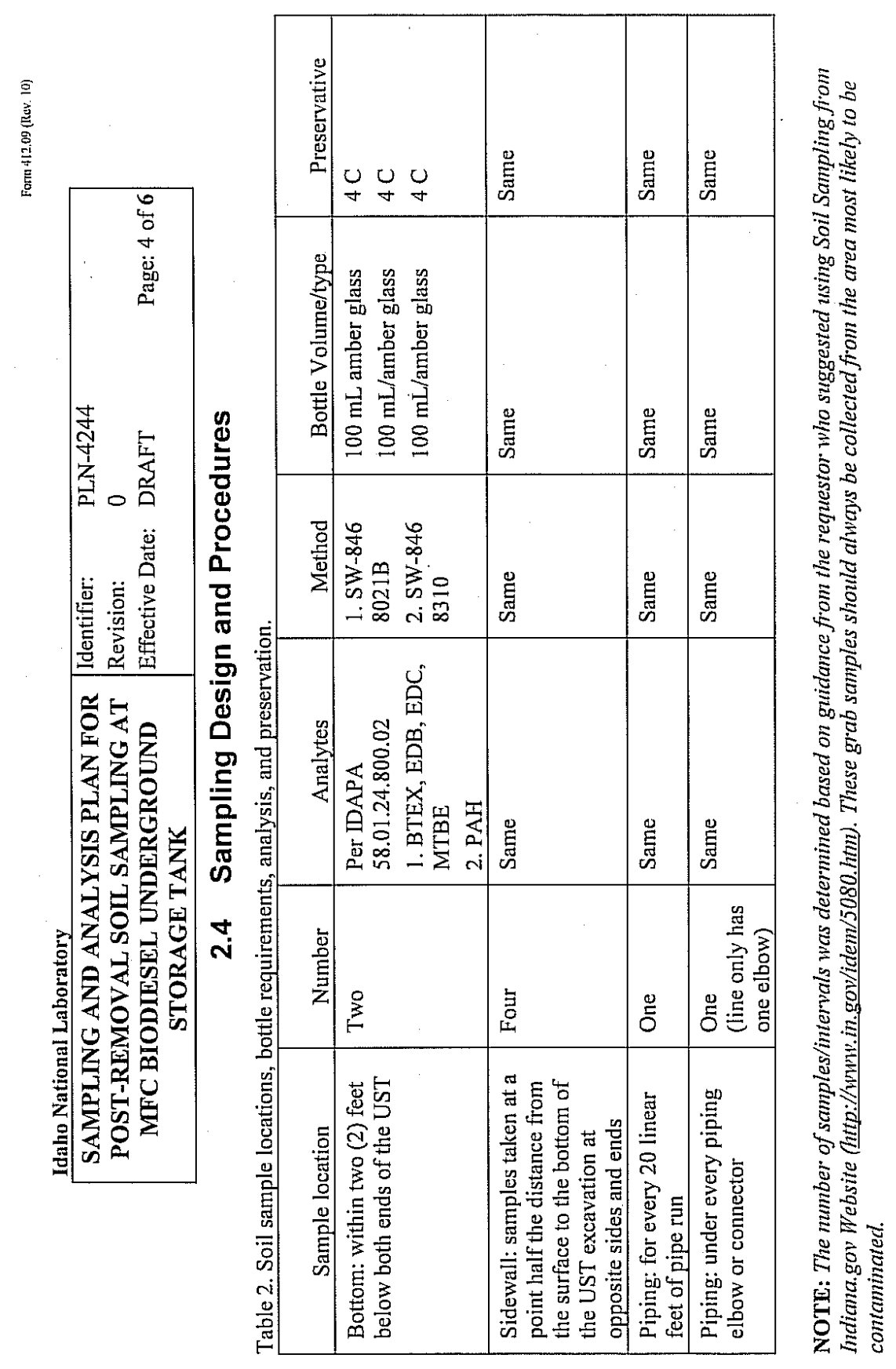




\begin{tabular}{|c|lll|}
\hline SAMPLING AND ANALYSIS PLAN FOR & Identifier: & PLN-4244 & \\
POST-REMOVAL SOIL SAMPLING AT & Revision: & 0 & \\
MFC BIODIESEL UNDERGROUND & Effective Date: & DRAFT & Page: 5 of 6 \\
STORAGE TANK & & & \\
\hline
\end{tabular}

\subsection{Waste Handling}

All sampling derived waste will be handled according to instructions from Kerry Nisson.

\section{DATA QUALITY REQUIREMENTS AND ASSESSMENTS}

\subsection{Data Completeness and Representativeness}

All samples collected during this activity will be prepared and analyzed according to guidelines set forth in the laboratory contract. It is imperative that the designated laboratory performs the analysis using these techniques to ensure data quality, precision, accuracy, and completeness. All sample collecting, handling, and analytical protocols have been chosen so the results will be as representative as possible of the media and conditions being measured.

\subsection{Quality Control Requirements}

QC has been discussed in Section 2.

\section{DOCUMENTATION}

\subsection{Sample Custody}

Full chain of custody will be maintained at all times, as specified in MCP-8523, "Managing Hazardous and Nonhazardous Samples."

\subsection{Data Reporting}

All analytical results, $\mathrm{COC}$, and QC measurements for each sample will be required from the laboratory with a turnaround time as contracted with the offsite laboratory. All data will be submitted to BEA with notification made to the Analytical Laboratory Contracting Lead: Peggy Scherbinske, BEA, via E-mail.

\section{WASTE DISPOSAL}

Any waste generated during sampling will be disposed of at the direction of Waste Generator Services (WGS). It is anticipated that discarded sampling equipment (decon towels, PPE) will be treated as industrial waste based upon previous similar sampling results.

\section{TRAINING}

Personal protective equipment (PPE) is called out in the project specific work order.

Minimum PPE to perform work activities involving handling of wastes includes; sturdy leather shoes above the ankle, safety glasses, and nitrile gloves. Any PPE different than or in addition to the minimum listed can be documented in the sample log notes. 
Form 412.09 (Rev. 10)

Idaho National Laboratory SAMPLING AND ANALYSIS PLAN FOR

POST-REMOVAL SOIL SAMPLING AT MFC BIODIESEL UNDERGROUND STORAGE TANK

Identifier: PLN-4244

Revision: 0

Effective Date: DRAFT

Appendix A

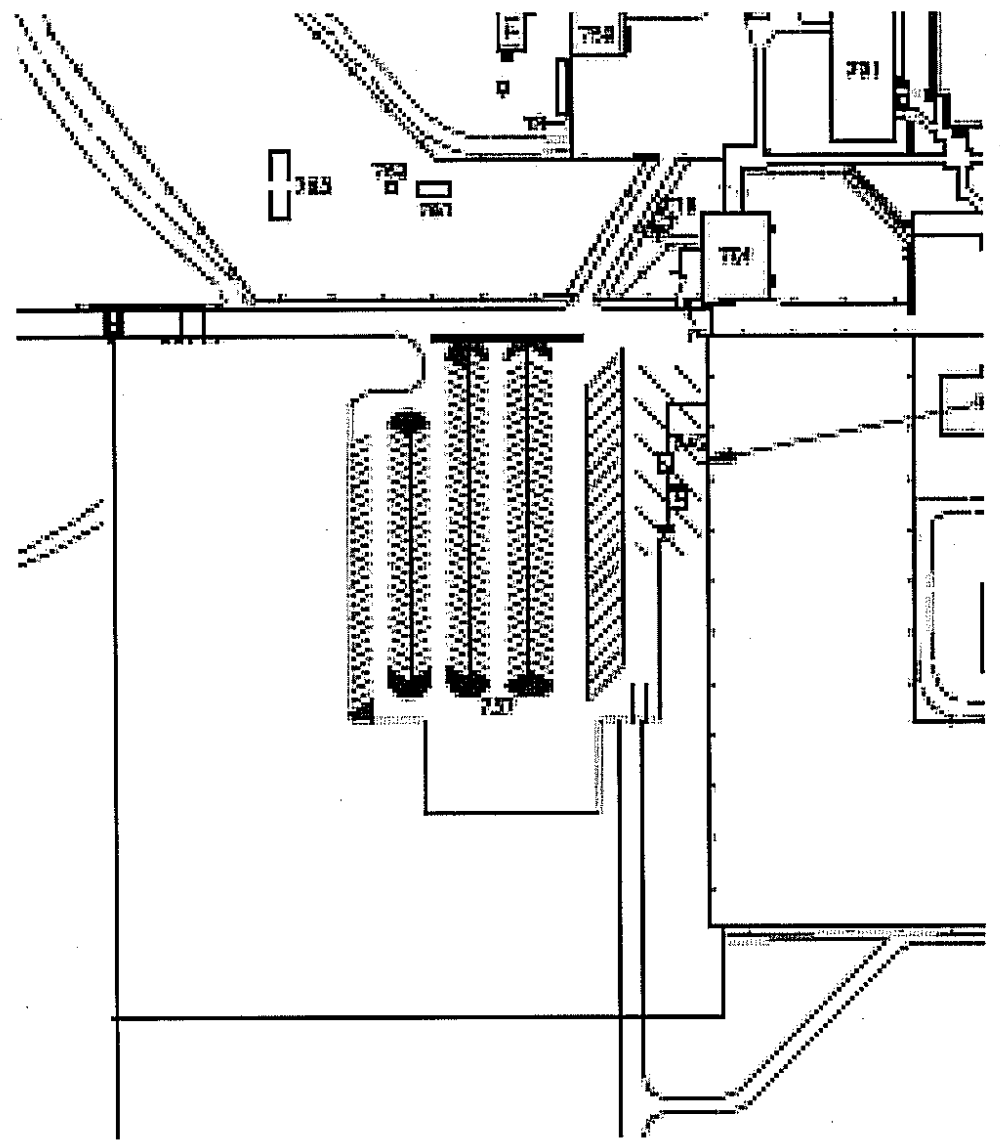

Figure 1. Plan map of MFC parking lot and UST area.

Page: 6 of 6 


\section{Appendix C \\ Notification of Underground Storage Tank Removal Correspondence (CCN 228291)}

Idaho National Laboratory Mail - CCN: 228291 Notification for underground storage tank removal at the... Page 1 of 1

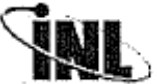

liono Noviced loberobin

Nisson, Kerry L <kerry.nisson@inl.gov>

CCN: 228291 Notification for underground storage tank removal at the INL MFC

1 message

Nisson, Kerry L <kerry.nisson@inl.gov>

Mon, Aug 27, 2012 at 9:58 AM

To: ENVIRONMENTAL CORRESPONDENCE ServiceID <envaff@inl.gov>, Timothy L Carlson <timothy.carlson@inl.gov>,

James F Graham <James.Graham@inl.gov>, Timothy A Miller <Timothy.Miller@inl.gov>, Scott L Lyman

<Scott.Lyman@inl.gov>, Lucien E Frederick Jr <Lucien.Frederick@inl.gov>

Cc: Bradley K Griffith <Bradley.Griffith@inl.gov>, Jason Sturm <STURMJR@id.doe.gov>, Jo A Stenzel <jo.stenzel@inl.gov>

Alan E Carvo<Alan.Carvo@inl.gov>, Lynne M Coe-Leavitt <Lynne.Coe-Leavitt@inl.gov>, Tim A Solle<Tim.Solle@inl.gov>,

Tom Haney <tom.haney@inl.gov>, Carlo D Melbihess <carlo.melbihess@inl.gov>, Reva A Nickelson <reva.nickelson@inl.gov>

On August 27, 2012 at 09:13, a conference call was placed to Steve Heaton (DEQ), by Jason Sturm (DOE-ID) and myself. The purpose of the call was to notify DEQ that the UST tank removal at MFC would begin this week, and to identify if DEQ wanted to perform any inspection. This call is a follow-up to the required 30-day notification of intent to close an underground storage tank (ref. CCN 227777) that was sent to DEQ on July 9, 2012.

Steve stated that DEQ did not plan to visit the INL during the UST removal and to keep him informed as the project progressed. I stated that DEQ would be notified if there were any evidence of tank leakage during the removal.

Jason also questioned if DEQ wanted to inspect the completed line replacements. Steve stated that DEQ wasn't planning to inspect and would just want to review the paperwork for the replaced lines.

Kerry L. Nisson

Nuclear Operations Environmental Support - UST TPOC

Office (208) 533-7102

Cell (208) 569-4721

email: kerry.nisson@inl.gov

Mail Stop 6134 


\section{Appendix D \\ Sample Analytical Report \#1}

\section{A}

September 19, 2012

Ms. Peggy Scherbinske

Battelle Energy Alliance

$2525 \mathrm{~N}$. Fremont Ave.

Idaho Falls, ID 83415-6194

Re: ALS Workorder: Project Name:

12-09-154

Project Number:

MFC Biodiesel Tank Removal, TOS-A1175

BEA030488

Dear Ms. Scherbinske:

Twelve soil samples were recelved from Battelle Energy Alliance on September 12, 2012. The samples were scheduled for the following analysis:

GC/MS Volatiles Pages 1-36

GC/MS Semivolatiles Pages 1-33

The results for this analysis are contained in the enclosed report.

Thank you for your confidence in ALS Environmental. Should you have any questions, please call.

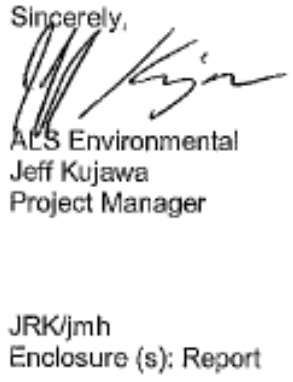

ADDRESS 225 Commerce Drive, Fort Collins, Colorado, USA BOS24 ? PHONE +1 9704901511 | FAX +1 9704901522 ALS Group USA, CORP. Part of the A.S Laboratary Croup A Campbell Brothers Limilted Compam

\section{wwwalsglobalicom}

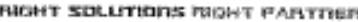


ALS is accredited by the following accreditation bodies for varlous testing scopes in accordance with requirements of each accreditation body. All testing is performed under the laboratory management system, which is maintained to meet these requirement and regulations. Please contact the laboratory or accreditation body for the current scope testing parameters.

\begin{tabular}{|c|c|}
\hline Washington & C1280 \\
\hline Utah & $\mathrm{CO00078}$ \\
\hline Arizona & $A Z 0742$ \\
\hline Alaska & UST-086 \\
\hline Alaska & 0000078 \\
\hline Florida & E87914 \\
\hline Missouri & 175 \\
\hline North, Dakota & $R-057$ \\
\hline New Jersey & $\mathrm{COO03}$ \\
\hline Nevada & CO000782008A \\
\hline California & $06251 \mathrm{CA}$ \\
\hline Kansas & $E-10381$ \\
\hline Maryland & 285 \\
\hline Pennsylvania & $68-03116$ \\
\hline Texas & T104704241-09-1 \\
\hline Colorado & Co00078 \\
\hline Connecticut: & $\mathrm{PH}=0232$ \\
\hline Idaho & 0000078 \\
\hline Tennessee & 2973 \\
\hline Kentucky & 90137 \\
\hline L-A-B (DoD ELAP/ISO 17025) & $\mathrm{L} 2257$ \\
\hline
\end{tabular}




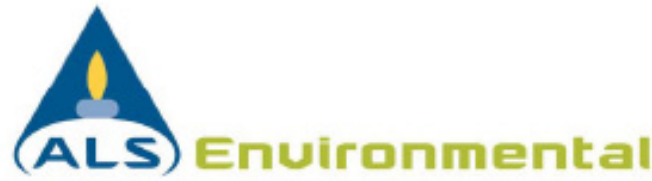

\section{GC/MS Semivolatiles SIMPAH \\ Case Narrative}

\section{Battelle Energy Alliance \\ MFC Biodiesel Tank Removal, TOS-A1175 -- BEA030488}

Work Order Number: 1209154

1. This report consists of 6 soil samples. The samples were received cool and intact by ALS on 09/12/12.

2. These samples were prepared and analyzed according to SW-846, 3rd Edition procedures. Specifically, the soil samples were extracted using soxhlet procedures according to SW-846 Method $3540 \mathrm{C}$ utilizing the current revision of SOP 625.

3. The extracts were analyzed using GC/MS with a DB-5MS capillary column according to the current revision of SOP 506 based on SW-846 Method 8270D. The samples were analyzed using selective ion monitoring (SIM), in order to achieve lower reporting limits. All positive results were quantitated against the initial calibration standards using the internal standard technique. The identification of positive results was achieved by a comparison of the retention time and a limited number of major ions from the mass spectrum of the sample versus the daily calibration standard.

4. All initial calibration criteria were met. If average response factors were used in the initial calibration, $\% R S D$ was $\leq 20 \%$. If linear or higher order regression calibrations were used in the initial calibration, the coefficient of determination $\left(r^{2}\right) \geq 0.99$.

5. All initial calibration standards are verified by comparing a second source standard initial calibration verification (ICV) against the calibration curve. All target compounds in the second source verification had $\mathrm{a} \% \mathrm{D} \leq 30 \%$.

6. All method blank criteria were met.

7. All laboratory control sample and laboratory control sample duplicate recoveries and RPDs were within the acceptance criteria. 
8. Sample 1209154-8 was designated as the quality control sample for this analysis. Similarity of matrix and therefore relevance of the QC results should not be automatically inferred for any sample other than the native sample selected for QC.

All matrix spike and matrix spike duplicate recoveries and RPDs were within acceptance criteria with the following exceptions:

\begin{tabular}{|c|c|c|}
\hline Spiked Compound & QC Sample & Direction \\
\hline Pyrene & MS/MSD & RPD \\
\hline Benzo(b)fluoranthene & MS/MSD & RPD \\
\hline
\end{tabular}

The recoveries of these compounds in the laboratory control sample and laboratory control sample duplicate were within control limits, which suggest the outliers in the matrix spikes may have been due to matrix effects, so no further action was taken.

9. The samples were extracted and analyzed within the established holding times.

10. All surrogate recoveries were within acceptance criteria.

11. All internal standard recoveries were within acceptance criteria.

12. Sample 1209154-6 was analyzed at dilution to bring target analytes in to calibration range. The reporting limits have been adjusted accordingly.

13. Manual integrations are performed when needed to provide consistent and defensible data following the guidelines in the current revision of SOP 939.

The data contained in the following report have been reviewed and approved by the personnel listed below. In addition, ALS certifies that the analyses reported herein are true, complete and correct within the limits of the methods employed.
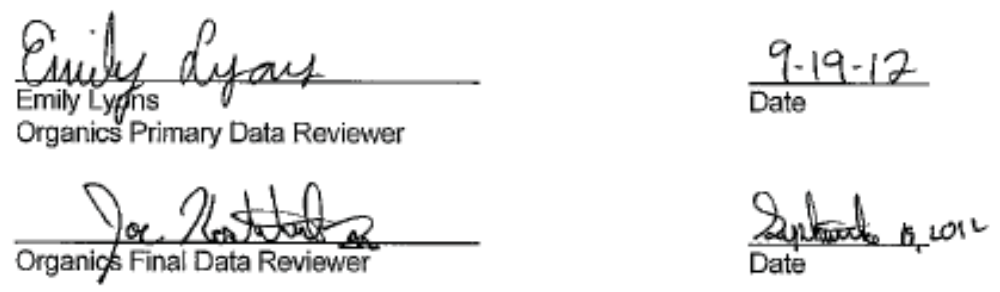


\title{
A
}

\author{
ALS \\ Data Qualifier Flags \\ Chromatography and Mass Spectrometry
}

U or ND: This flag indicates that the compound was analyzed for but not detected.

$\mathrm{J}$ This flag indicates an estimated value. This flag is used as follows: (1) when estimating a concentration for tentatively identified compounds (TICs) where a 1:1 response is assumed; (2) when the mass spectral and retention time data indicate the presence of a compound that meets the volatile and semivolatile GC/MS identification criteria, and the result is less than the reporting limit (RL) but greater than the method detection limit (MDL); (3) when the retention time data indicate the presence of a compound that meets the GC identification criteria, and the result is less than the RL but greater than the MDL; and (4) the reported value is estimated.

B: $\quad$ This flag is used when the analyte is detected in the associated method blank as well as in the sample. It indicates probable blank contamination and warns the data user. This flag shall be used for a tentatively identified compound (TIC) as well as for a positively identified target compound.

E: $\quad$ This flag identifies compounds whose concentration exceeds the upper level of the calibration range.

A: $\quad$ This flag indicates that a tentatively identified compound is a suspected aldol-condensation product.

X: $\quad$ This flag indicates that the analyte was diluted below an accurate quantitation level.

*: $\quad$ This flag indicates that a spike recovery is equal to or outside the control criteria used.

+: $\quad$ This flag indicates that the relative percent difference (RPD) equals or exceeds the control criteria. 


\section{Ats}

\section{Chain of Custody}

4 of 33 


\section{ALS Environmental -- FC \\ Sample Number(s) Cross-Reference Table}

\begin{tabular}{|c|c|c|c|c|c|}
\hline \multicolumn{6}{|c|}{$\begin{aligned} & \text { OrderNum: } 1209154 \\
& \text { Client Name: Battelle Energy Alliance } \\
& \text { Client Project Name: MFC Biodiesel Tank Removal ,TOS-A1175 } \\
& \text { Client Project Number: BEA030488 } \\
& \text { Client PO Number: SOW-8500 }\end{aligned}$} \\
\hline $\begin{array}{c}\text { Client Sample } \\
\text { Number }\end{array}$ & $\begin{array}{c}\text { Lab Sample } \\
\text { Number }\end{array}$ & COC Number & Matrix & \begin{tabular}{|c|} 
Date \\
Collected
\end{tabular} & $\begin{array}{c}\text { Time } \\
\text { Collected }\end{array}$ \\
\hline BEA030488_MFC & $1209154-1$ & & SOIL & 10-Sep-12 & $14: 36$ \\
\hline BEA030489_MFC & $1209154-2$ & & SOIL & 10-Sep-12 & $14: 36$ \\
\hline BEA030490_MFC & $1209154-3$ & & SOIL & $10-$ Sep-12 & $14: 22$ \\
\hline BEA030491_MFC & $1209154-4$ & & SOIL & $10-$ Sep-12 & $14: 22$ \\
\hline BEA030492_MFC & $1209154-5$ & & SOIL & $10-$ Sep-12 & $13: 41$ \\
\hline BEA030493_MFC & $1209154-6$ & & SOIL & 10-Sep-12 & $13: 41$ \\
\hline BEA030494_MFC & $1209154-7$ & & SOIL & 10-Sep-12 & $13: 55$ \\
\hline BEA030495_MFC & $1209154-8$ & & SOIL & $10-$ Sep-12 & $13: 55$ \\
\hline BEA030496_MFC & $1209154-9$ & & SOIL & 10-Sep-12 & $14: 44$ \\
\hline BEA030497_MFC & $1209154-10$ & & SOIL & $10-$ Sep-12 & $14: 44$ \\
\hline BEA030498_MFC & $1209154-11$ & & SOIL & $10-$ Sep-12 & $14: 28$ \\
\hline BEA030499_MFC & $1209154-12$ & & SOIL & 10-Sep-12 & $14: 28$ \\
\hline
\end{tabular}




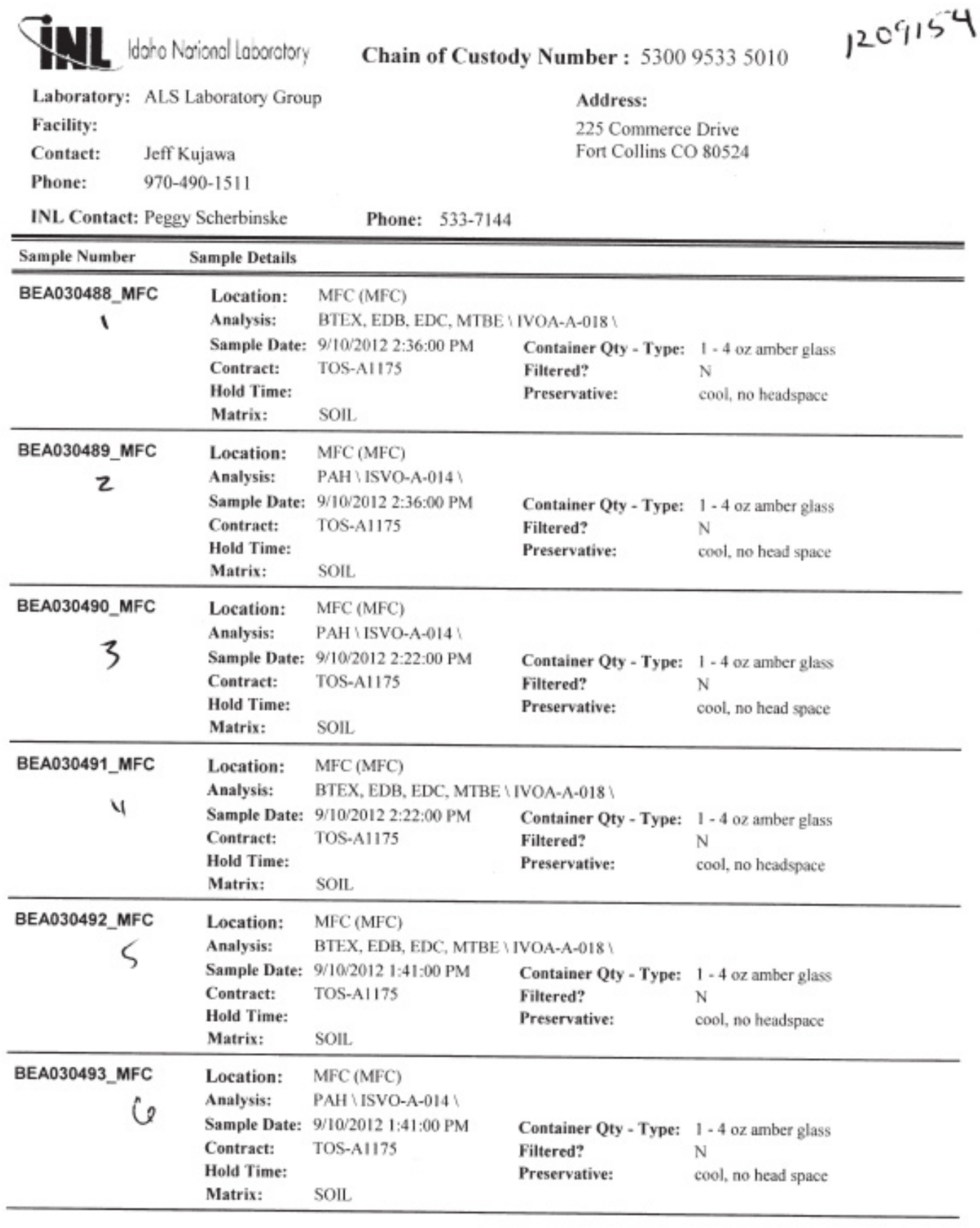




\begin{tabular}{|c|c|c|c|c|}
\hline \multirow[b]{2}{*}{$\begin{array}{c}\text { BEA030494_MFC } \\
-1\end{array}$} & nol Loborctory & \multicolumn{3}{|c|}{ Chain of Custody Number : 530095335010} \\
\hline & $\begin{array}{l}\text { Location: } \\
\text { Analysis: } \\
\text { Sample Date: } \\
\text { Contract: } \\
\text { Hold Time: } \\
\text { Matrix: }\end{array}$ & $\begin{array}{l}\text { MFC (MFC) } \\
\text { PAH \ISVO-A-014 } \\
9 / 10 / 20121: 55: 00 \mathrm{PM} \\
\text { TOS-A1175 } \\
\text { SOIL }\end{array}$ & $\begin{array}{l}\text { Container Qty - Type: } \\
\text { Filtered? } \\
\text { Preservative: }\end{array}$ & $\begin{array}{l}\text { I - } 4 \text { oz amber glass } \\
\mathrm{N} \\
\text { cool, no head space }\end{array}$ \\
\hline BEA030495_MFC & $\begin{array}{l}\text { Location: } \\
\text { Analysis: } \\
\text { Sample Date: } \\
\text { Contract: } \\
\text { Hold Time: } \\
\text { Matrix: }\end{array}$ & $\begin{array}{l}\text { MFC (MFC) } \\
\text { BTEX, EDB, EDC, MT1 } \\
\text { 9/10/2012 1:55:00 PM } \\
\text { TOS-A1175 } \\
\text { SOIL }\end{array}$ & $\begin{array}{l}\text { [VOA-A-018 } \\
\text { Container Qty - Type: } \\
\text { Filtered? } \\
\text { Preservative: }\end{array}$ & $\begin{array}{l}\text { I- } 4 \text { oz amber glass } \\
\mathrm{N} \\
\text { cool, no headspace }\end{array}$ \\
\hline $\begin{array}{c}\text { BEA030496_MFC } \\
9\end{array}$ & $\begin{array}{l}\text { Location: } \\
\text { Analysis: } \\
\text { Sample Date: } \\
\text { Contract: } \\
\text { Hold Time: } \\
\text { Matrix: }\end{array}$ & $\begin{array}{l}\text { MFC (MFC) } \\
\text { BTEX, EDB, EDC. MTI } \\
\text { 9/10:2012 2:44:00 PM } \\
\text { TOS-A1175 } \\
\text { SOIL. }\end{array}$ & $\begin{array}{l}\text { IVOA-A-018 } \\
\text { Container Qty - Type: } \\
\text { Filtered? } \\
\text { Preservative: }\end{array}$ & $\begin{array}{l}\text { 1-4 oz amber glass } \\
\mathrm{N} \\
\text { cool, no headspace }\end{array}$ \\
\hline $\begin{array}{c}\text { BEA030497_MFC } \\
, 0\end{array}$ & $\begin{array}{l}\text { Location: } \\
\text { Analysis: } \\
\text { Sample Date: } \\
\text { Contract: } \\
\text { Hold Time: } \\
\text { Matrix: }\end{array}$ & $\begin{array}{l}\text { MFC (MFC) } \\
\text { PAH } \backslash 1 S V O-A=014 \backslash \\
9 / 10 / 20122: 44: 00 \mathrm{PM} \\
\text { TOS-A1175 } \\
\text { SOIL. }\end{array}$ & $\begin{array}{l}\text { Container Qty - Type: } \\
\text { Filtered? } \\
\text { Preservative: }\end{array}$ & $\begin{array}{l}1-4 \mathrm{oz} \text { amber glass } \\
\mathrm{N} \\
\text { cool, no head space }\end{array}$ \\
\hline BEA030498_MFC & $\begin{array}{l}\text { Location: } \\
\text { Analysis: } \\
\text { Sample Date: } \\
\text { Contract: } \\
\text { Hold Time: } \\
\text { Matrix: }\end{array}$ & $\begin{array}{l}\text { MFC (MFC) } \\
\text { PAH } \backslash \text { ISVO-A-014 } \\
\text { 9/10/2012 2:28:00 PM } \\
\text { TOS-A1175 } \\
\text { SOIL }\end{array}$ & $\begin{array}{l}\text { Container Qty - Type: } \\
\text { Filtered? } \\
\text { Preservative: }\end{array}$ & $\begin{array}{l}\text { 1-4 oz amber glass } \\
\mathrm{N} \\
\text { cool, no head space }\end{array}$ \\
\hline BEA030499_MFC & $\begin{array}{l}\text { Location: } \\
\text { Analysis: } \\
\text { Sample Date: } \\
\text { Contract: } \\
\text { Hold Time: } \\
\text { Matrix: }\end{array}$ & $\begin{array}{l}\text { MFC (MFC) } \\
\text { BTEX, EDB, EDC, MTt } \\
\text { 9/10/2012 2:28:00 PM } \\
\text { TOS-A1175 } \\
\text { SOIL }\end{array}$ & $\begin{array}{l}\text { IVOA-A-018 } \\
\text { Container Qty - Type: } \\
\text { Filtered? } \\
\text { Preservative: }\end{array}$ & $\begin{array}{l}1-4 \text { oz amber glass } \\
\mathrm{N} \\
\text { cool, no headspace }\end{array}$ \\
\hline
\end{tabular}


L doho Notionol Loborciory Chain of Custody Number : 530095335010

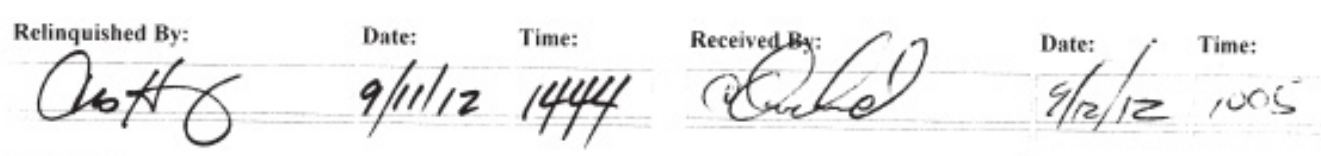

Comments:

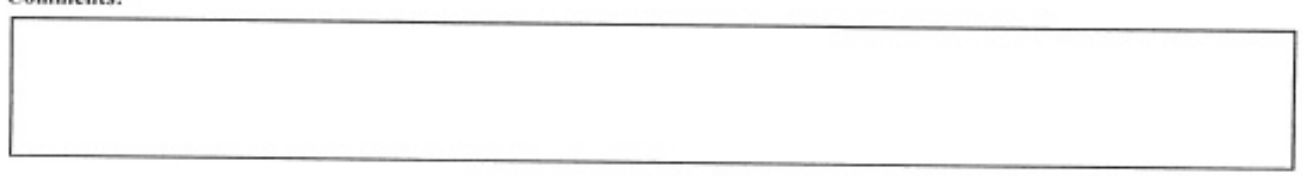

Monday, September 10, 2012

Pageof'33

37 


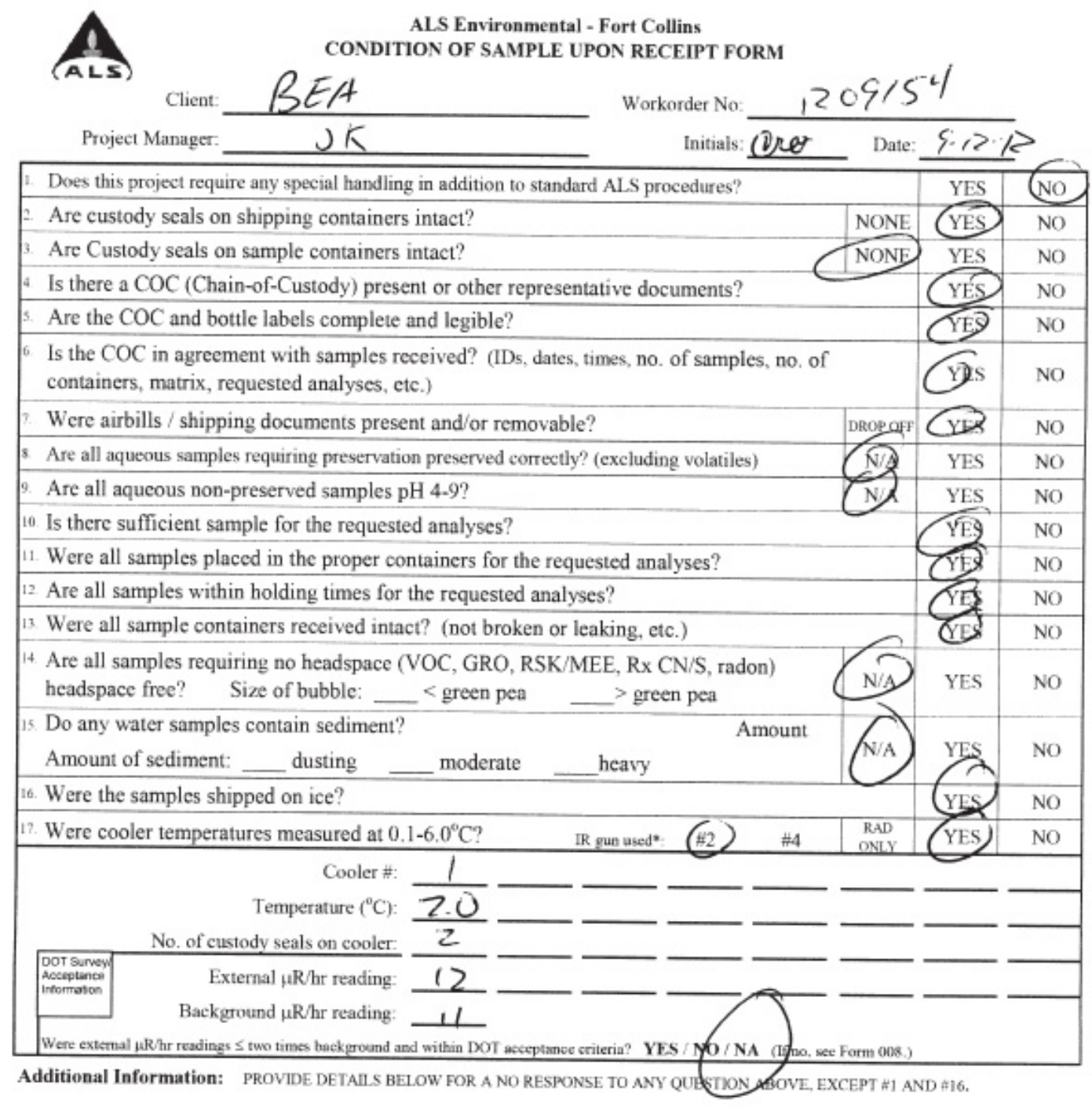

If applicnble, was the client contacted? YES / NO NA Contact:

Projeet Manager Signature / Date:

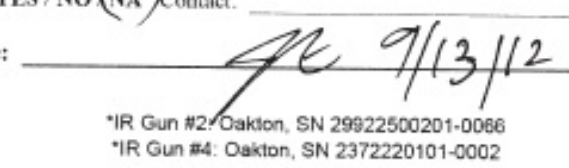

Date Time:

Form 201r24.xls $(0604 / 2012)$

IR Gun 24: Oakton, SN 2372220101-0002

9 of $3 \$$ 


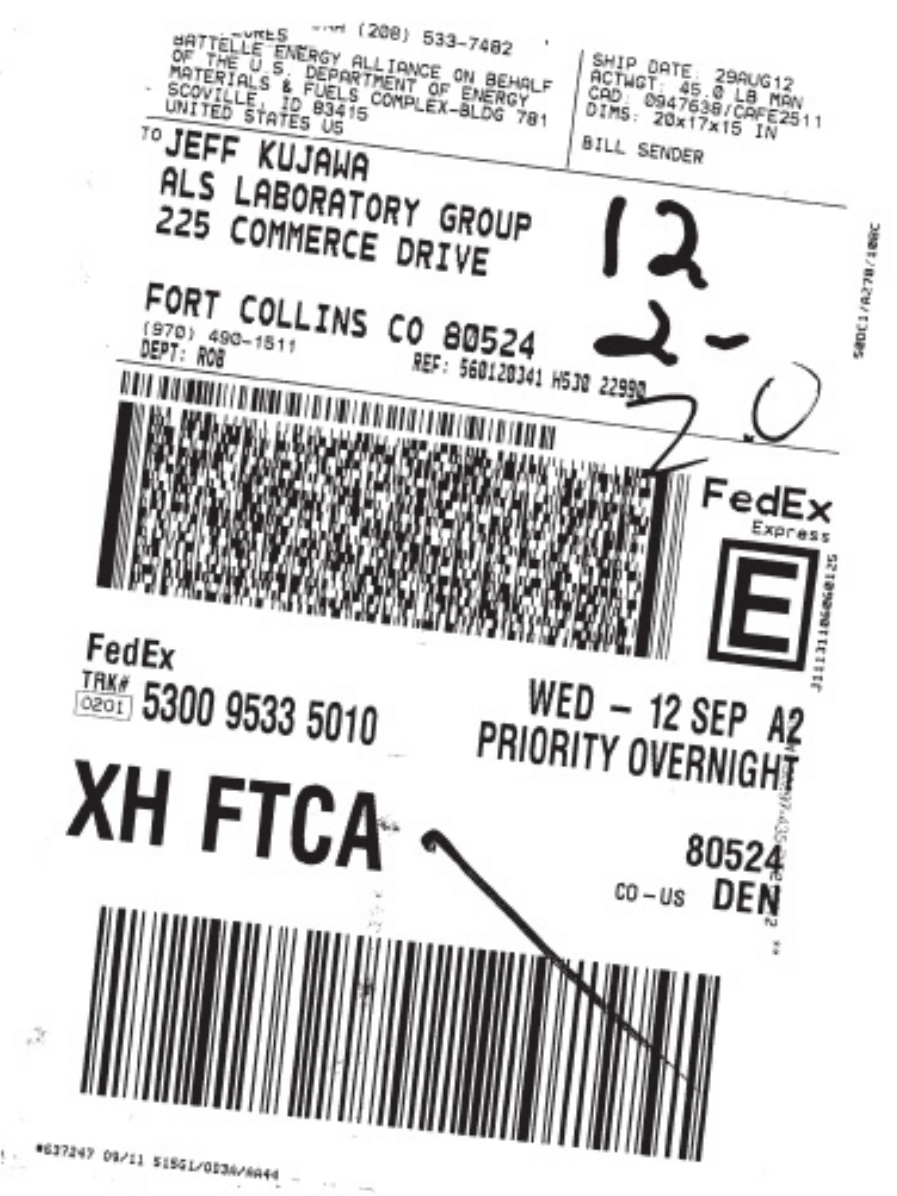

10 of 33 


\section{A}

\section{Analytical Results}

11 of 33 


\title{
GC/MS Semi-volatiles
}

\author{
Method SW8270SIMPAHD \\ Method Blank
}

Lab Name: ALS Environmental - FC

Work Order Number: 1209154

Client Name: Battele Energy Alliance

ClientProject ID: MFC Biodiesel Tank Removal ,TOS-A1175 BEA030488

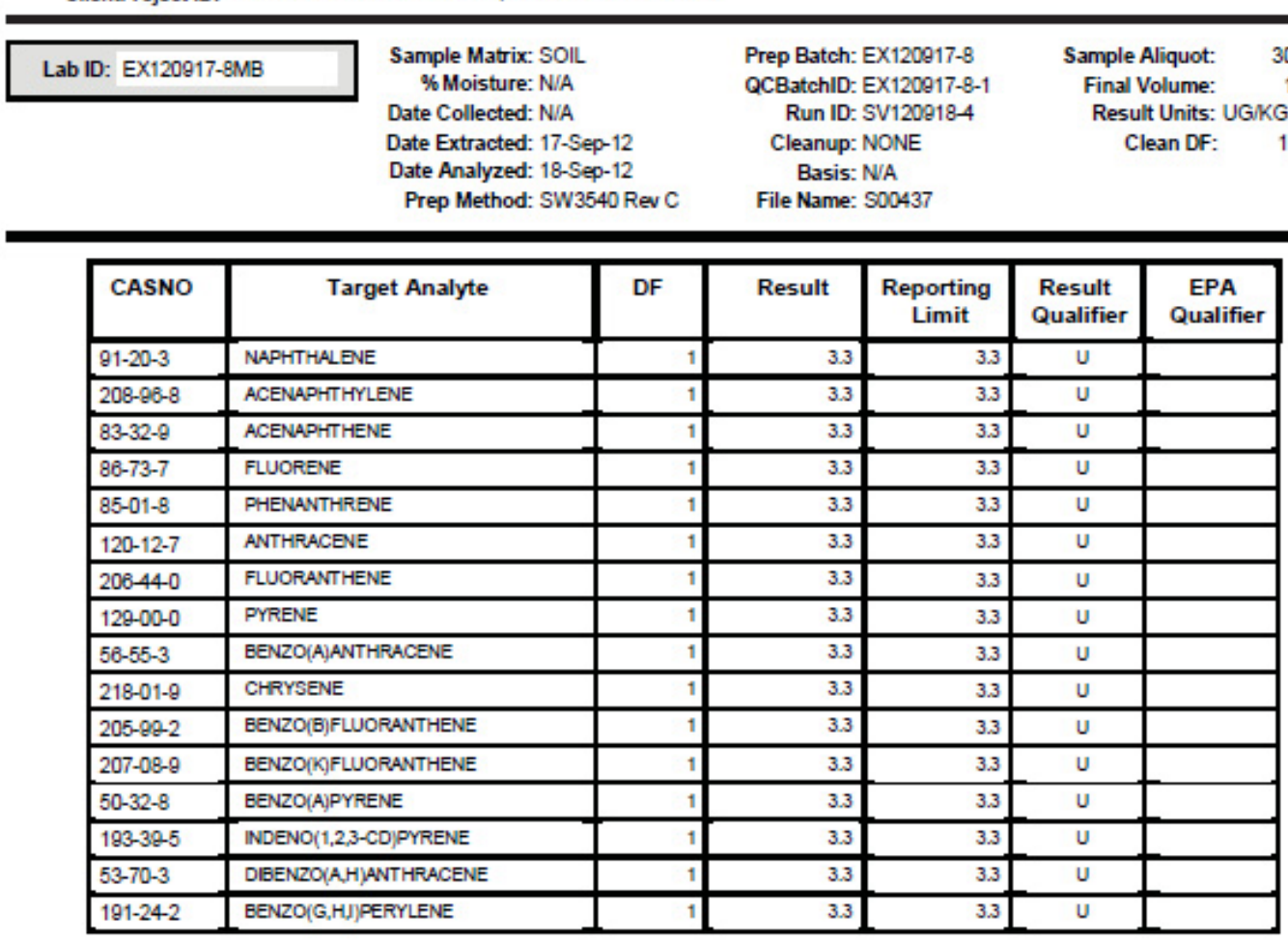

Surrogate Recovery

\begin{tabular}{|l|l|r|r|r|r|c|}
\hline CASNO & \multicolumn{1}{|c|}{ Surrogate Analyte } & Result & Flag & $\begin{array}{c}\text { Spike } \\
\text { Amount }\end{array}$ & $\begin{array}{c}\text { Percent } \\
\text { Recovery }\end{array}$ & $\begin{array}{c}\text { Control } \\
\text { Limits }\end{array}$ \\
\hline $321-60-8$ & 2-FLUOROBIPHENYL & 56.1 & & 66.7 & 83 & $41-106$ \\
\hline $4165-60-0$ & NTRROBENZENE-DS & 56.2 & & 66.7 & 84 & $28-113$ \\
\hline $1718-51-0$ & TERPHENYL-D14 & 63.5 & & 66.7 & 85 & $25-147$ \\
\hline
\end{tabular}

Data Package ID: SV1209154-1

$\begin{array}{lcc}\text { Date Printed: Wednesday, September 19, } 2012 & \text { ALS Environmental -- FC } \\ \text { LMS veraion: 6.511 } & \text { Page } 1 \text { of } 1\end{array}$

${ }_{120633}$ 


\section{GC/MS Semi-volatiles}

\section{Method SW8270SIMPAH Revision D \\ Sample Results}

Lab Name: ALS Environmental - FC

Work Order Number: 1209154

Client Name: Battele Energy Alliance

ClientProject ID: MFC Biodiesel Tank Removal ,TOS-A1175 BEA030488

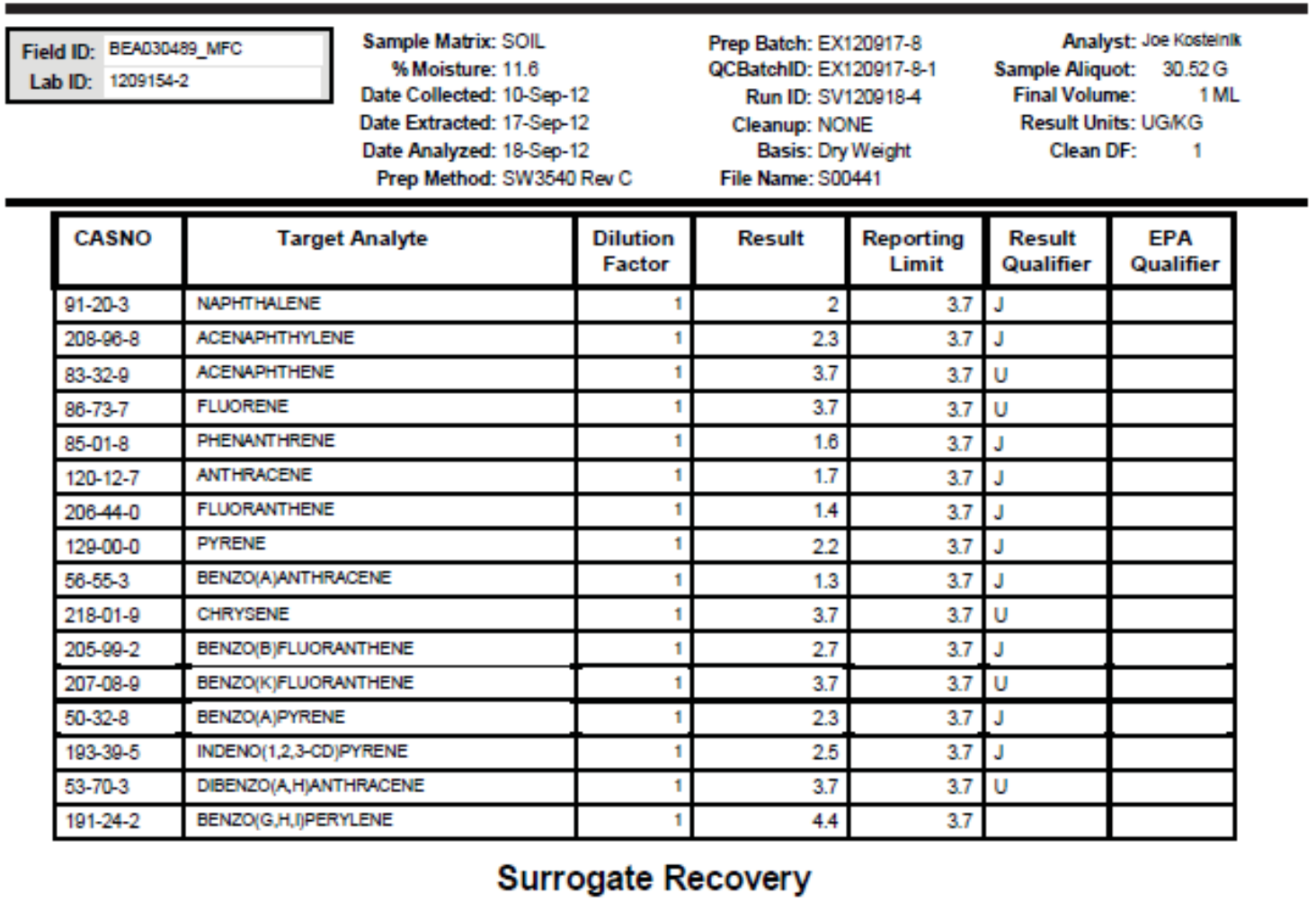

\begin{tabular}{|l|l|r|r|r|r|c|}
\hline CASNO & \multicolumn{1}{|c|}{ Surrogate Analyte } & Result & Flag & $\begin{array}{c}\text { Spike } \\
\text { Amount }\end{array}$ & $\begin{array}{c}\text { Percent } \\
\text { Recovery }\end{array}$ & $\begin{array}{c}\text { Control } \\
\text { Limits }\end{array}$ \\
\hline $321-60-8$ & 2-FLUOROBIPHENYL & 54.6 & & 74.2 & 74 & $41-106$ \\
\hline $4165-60-0$ & NITROEENZENE-D5 & 61.9 & & 74.2 & 84 & $28-113$ \\
\hline $1718-51-0$ & TERPHENYL-D14 & 69.8 & & 742 & 94 & $25-147$ \\
\hline
\end{tabular}

Data Package ID: SV1209154-1

Date Printed: Wedinesday, September 19, 2012

ALS Environmental -. FC

LMS Version: 6.611
Page 1 of 7

13 of 33 


\section{GC/MS Semi-volatiles}

\section{Method SW8270SIMPAH Revision D \\ Sample Results}

Lab Name: ALS Environmental - FC

Work Order Number: 1209154

Client Name: Battele Energy Alliance

ClientProject ID: MFC Biodiesel Tank Removal ,TOS-A1175 BEA030488

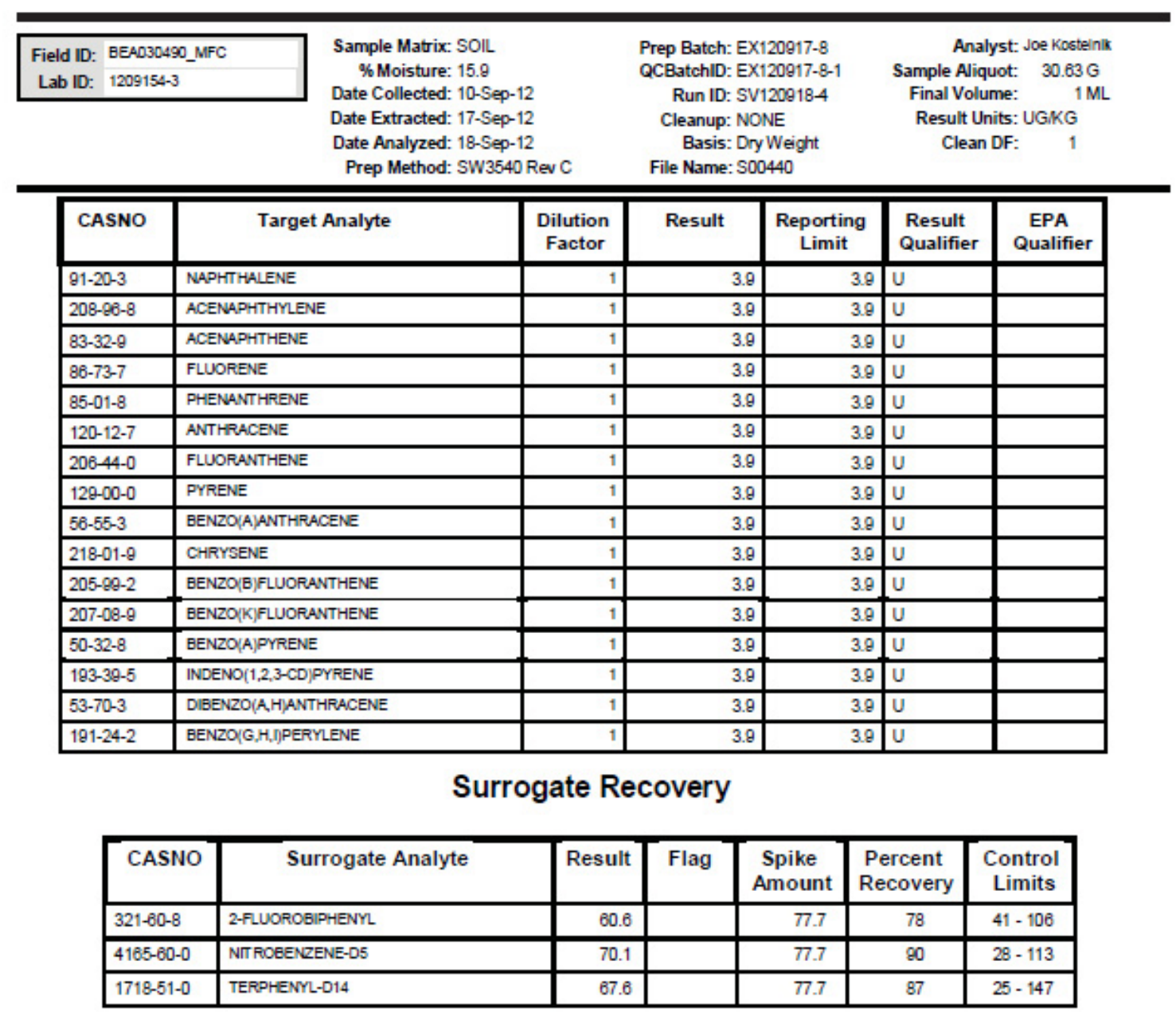

Data Package ID: SV1209154-1

$\begin{array}{cc}\text { Date Printed: Wednesday, September 19, } 2012 & \text { ALS Environmental -- FC } \\ \text { LMS Version: } 6.611 & \text { Page } 2 \text { of } 7\end{array}$

14 of 33 


\title{
GC/MS Semi-volatiles
}

\author{
Method SW8270SIMPAH Revision D \\ Sample Results
}

Lab Name: ALS Environmental - FC

Work Order Number: 1209154

Client Name: Battele Energy Alliance

ClientProject ID: MFC Biodiesel Tank Removal ,TOS-A1175 BEA030488

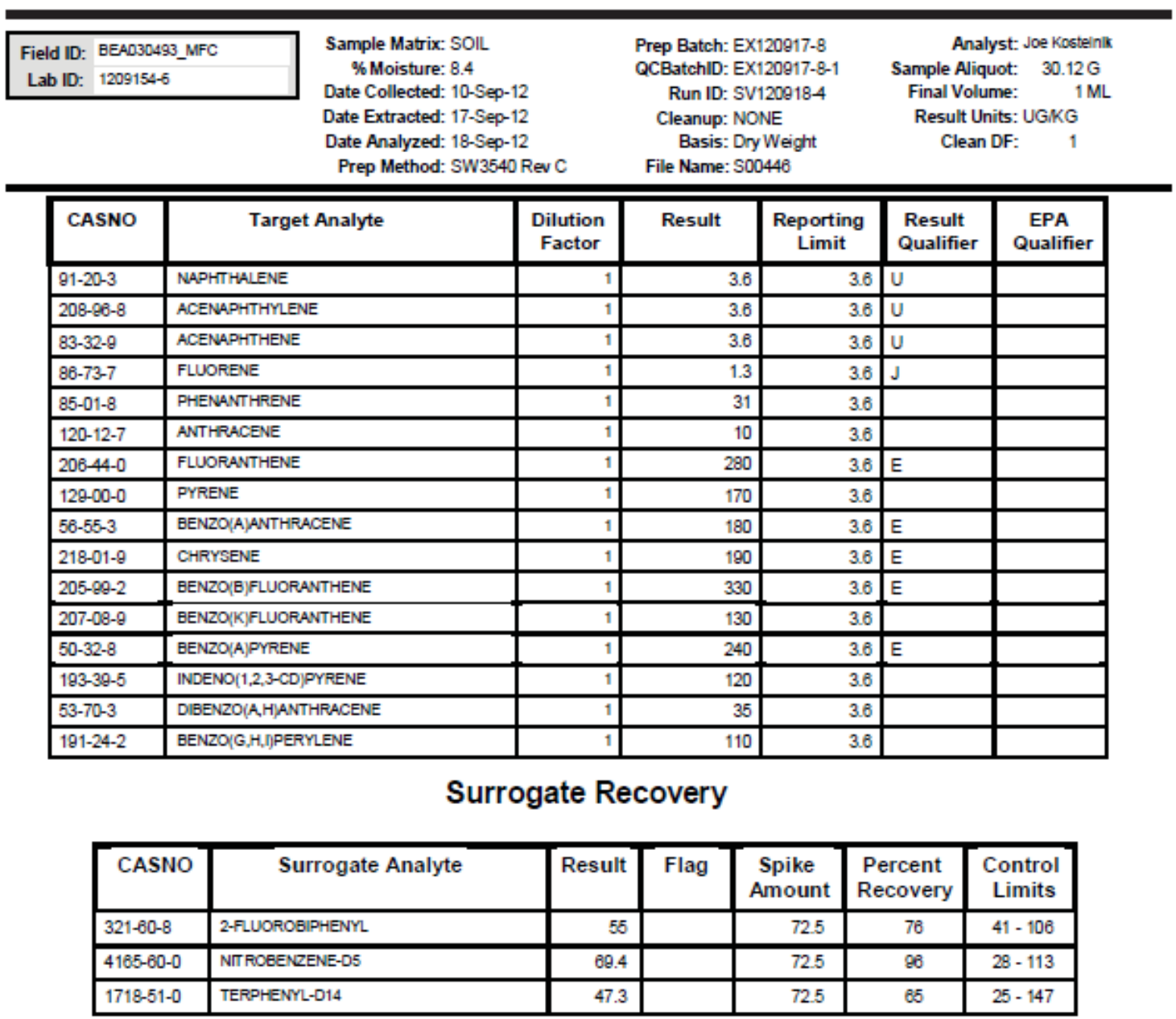

Data Package ID: SV1209154-1

$\begin{array}{lcc}\text { Date Printed: Wednesday, September 19, } 2012 & \text { ALS Environmental -- FC } \\ \text { LMS Version: } 6.611 & \text { Page } 3 \text { of } 7\end{array}$

15 of 33 


\section{GC/MS Semi-volatiles}

\section{Method SW8270SIMPAH Revision D \\ Sample Results}

Lab Name: ALS Environmental - FC

Work Order Number: 1209154

Client Name: Battele Energy Alliance

ClientProject ID: MFC Biodiesel Tank Removal ,TOS-A1175 BEA030488

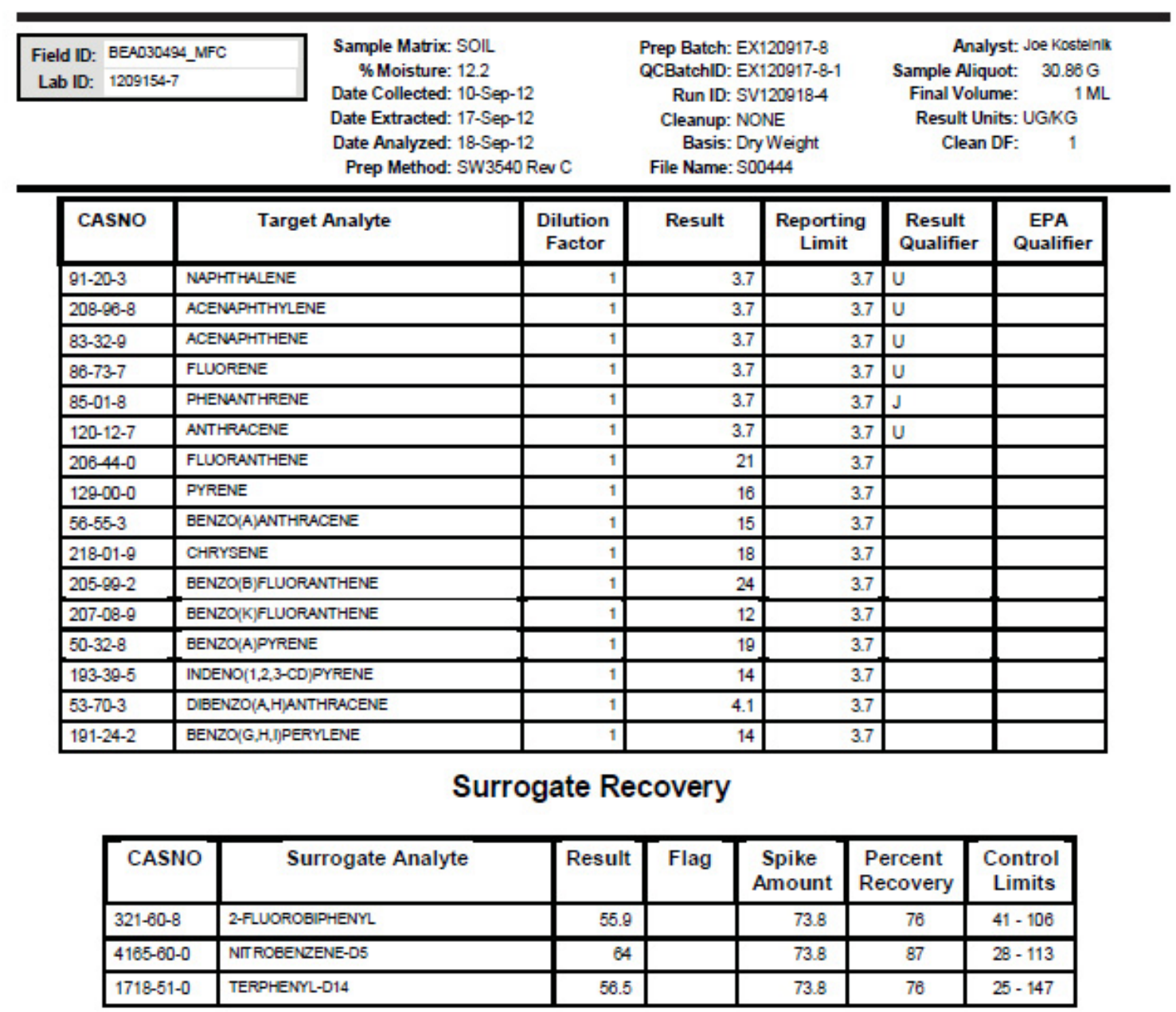

Data Package ID: SV1209154-1

$\begin{array}{cc}\text { Date Printed: Wednesday, September 19, 2012 ALS Environmental -- FC } & \text { Page } 4 \text { of } 7 \\ \text { LMS Version: } 6.611\end{array}$

16 of 33 


\section{Method SW8270SIMPAH Revision D}

Sample Results

Lab Name: ALS Environmental - FC

Work Order Number: 1209154

Client Name: Battele Energy Alliance

ClientProject ID: MFC Biodiesel Tank Removal ,TOS-A1175 BEA030488

\begin{tabular}{|c|c|c|c|c|c|c|}
\hline \multirow[t]{3}{*}{$\begin{aligned} \text { Field ID: } & \text { BEAD3 } \\
\text { Lab ID: } & 120915\end{aligned}$} & $\begin{array}{r}\text { Sample } \\
\% \mathrm{M} \\
\text { Date Co }\end{array}$ & \multirow{3}{*}{$\begin{array}{l}\text { Sample Matrix: SOIL } \\
\quad \% \text { Moisture: } 11.4 \\
\text { Date Collected: } 10-\text { Sep-12 } \\
\text { Date Extracted: } 17-\text { Sep-12 } \\
\text { Date Analyzed: } 18-S e p-12 \\
\text { Prep Method: SW3540 Rev C }\end{array}$} & \multirow{3}{*}{\multicolumn{2}{|c|}{$\begin{array}{l}\text { Prep Batch: EX120917-8 } \\
\text { QCBatchID: EX120917-8-1 } \\
\text { Run ID: SV120918-4 } \\
\text { Cleanup: NONE } \\
\text { Basis: DryWeight } \\
\text { File Name: S00447 }\end{array}$}} & \multicolumn{2}{|c|}{\begin{tabular}{lr}
\multicolumn{2}{c}{ Analyst: Joe Kosteink } \\
ample Aliquot: & $30.63 \mathrm{G}$ \\
Final Volume: & $1 \mathrm{ML}$
\end{tabular}} \\
\hline & Date Ext & & & & \multirow{2}{*}{\multicolumn{2}{|c|}{$\begin{array}{l}\text { Result Units: UGKG } \\
\text { Clean DF: }\end{array}$}} \\
\hline & $\begin{array}{l}\text { Date An } \\
\text { Prep : }\end{array}$ & & & & & \\
\hline CASNO & Target Analyte & $\begin{array}{l}\text { Dilution } \\
\text { Factor }\end{array}$ & Result & $\begin{array}{c}\text { Reporting } \\
\text { Limit }\end{array}$ & $\begin{array}{l}\text { Result } \\
\text { Qualifier }\end{array}$ & $\begin{array}{c}\text { EPA } \\
\text { Qualifier }\end{array}$ \\
\hline $91-20-3$ & NAPHTHALENE & 1 & 3.7 & 3.7 & $u$ & \\
\hline $208-86-8$ & ACENAPHTHYLENE & 1 & 3.7 & 3.7 & $U$ & \\
\hline $83-32-9$ & ACENAPHTHENE & 1 & 3.7 & 3.7 & 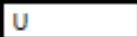 & \\
\hline $86-73-7$ & FLUORENE & 1 & 1.7 & 3.7 & $\mathrm{~J}$ & \\
\hline $85-01-8$ & PHENANTHRENE & 1 & 10 & 3.7 & & \\
\hline $120-12-7$ & ANTHRACENE & 1 & 3.1 & 3.7 & $\mathrm{~J}$ & \\
\hline $206-44-0$ & FLUORANTHENE & 1 & 40 & 3.7 & & \\
\hline $129-00-0$ & PYRENE & 1 & 30 & 3.7 & & \\
\hline $56-55-3$ & BENZOYA]ANTHRACENE & 1 & 30 & 3.7 & & \\
\hline 218-01-9 & CHRYSENE & 1 & 35 & 3.7 & & \\
\hline $205-89-2$ & BENZO(B)FLUORANTHENE & 1 & 56 & 3.7 & & \\
\hline $207-08-9$ & BENZO|KJFLUUORANTHENE & 1 & 22 & 3.7 & & \\
\hline $50-32-8$ & BENZO|A]PYRENE & 1 & 40 & 3.7 & & \\
\hline $193-39-5$ & INDENO(1,2,3-CD)PYRENE & 1 & 22 & 3.7 & & \\
\hline $53-70-3$ & DIBENZO(A,H)ANTHRACENE & 1 & 6.4 & 3.7 & & \\
\hline $191-24-2$ & BENZO(G,H,D)PERYLENE & 1 & 21 & 3.7 & & \\
\hline
\end{tabular}

Surrogate Recovery

\begin{tabular}{|l|l|r|r|r|r|c|}
\hline CASNO & \multicolumn{1}{|c|}{ Surrogate Analyte } & Result & Flag & $\begin{array}{c}\text { Spike } \\
\text { Amount }\end{array}$ & $\begin{array}{c}\text { Percent } \\
\text { Recovery }\end{array}$ & $\begin{array}{c}\text { Control } \\
\text { Limits }\end{array}$ \\
\hline $321-60-8$ & 2-FLUOROBIPHENYL & 60.8 & & 73.7 & 82 & $41-106$ \\
\hline $4165-60-0$ & NTROEENZENE-D5 & 77.1 & & 73.7 & 105 & $28-113$ \\
\hline $1718-51-0$ & TERPHENYL-D14 & 55.1 & & 73.7 & 75 & $25-147$ \\
\hline
\end{tabular}

Data Package ID: SV1209154-1

$\begin{array}{lcc}\text { Date Printed: Wednesday, September 19, } 2012 & \text { ALS Environmental -- FC } \\ \text { LMS Version: } 6.611 & \text { Page } 5 \text { of } 7\end{array}$

17 of 33 


\section{GC/MS Semi-volatiles}

\section{Method SW8270SIMPAH Revision D \\ Sample Results}

Lab Name: ALS Environmental - FC

Work Order Number: 1209154

Client Name: Battele Energy Alliance

ClientProject ID: MFC Biodiesel Tank Removad ,TOS-A1175 BEA030488

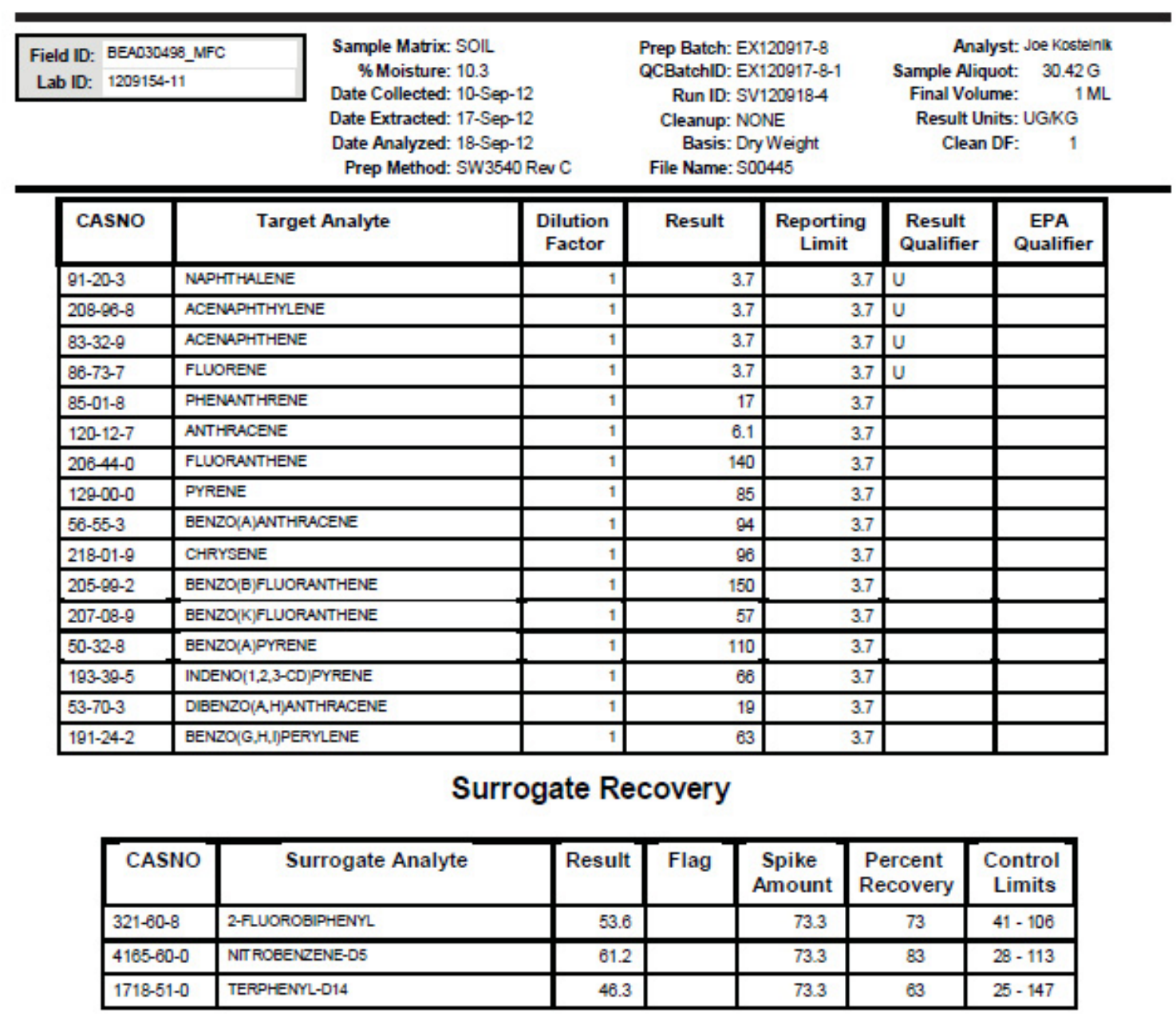

Data Package ID: SV1209154-1

$\begin{array}{cc}\text { Date Printed: Wednesday, September 19, } 2012 & \text { ALS Environmental -- FC } \\ \text { LMS version: } 6.611\end{array}$

18 of 33 
Lab Name: ALS Environmental - FC

Work Order Number: 1209154

Client Name: Battele Energy Alliance

ClientProject ID: MFC Biodiesel Tank Remova ,TOS-A1175 BEA030488

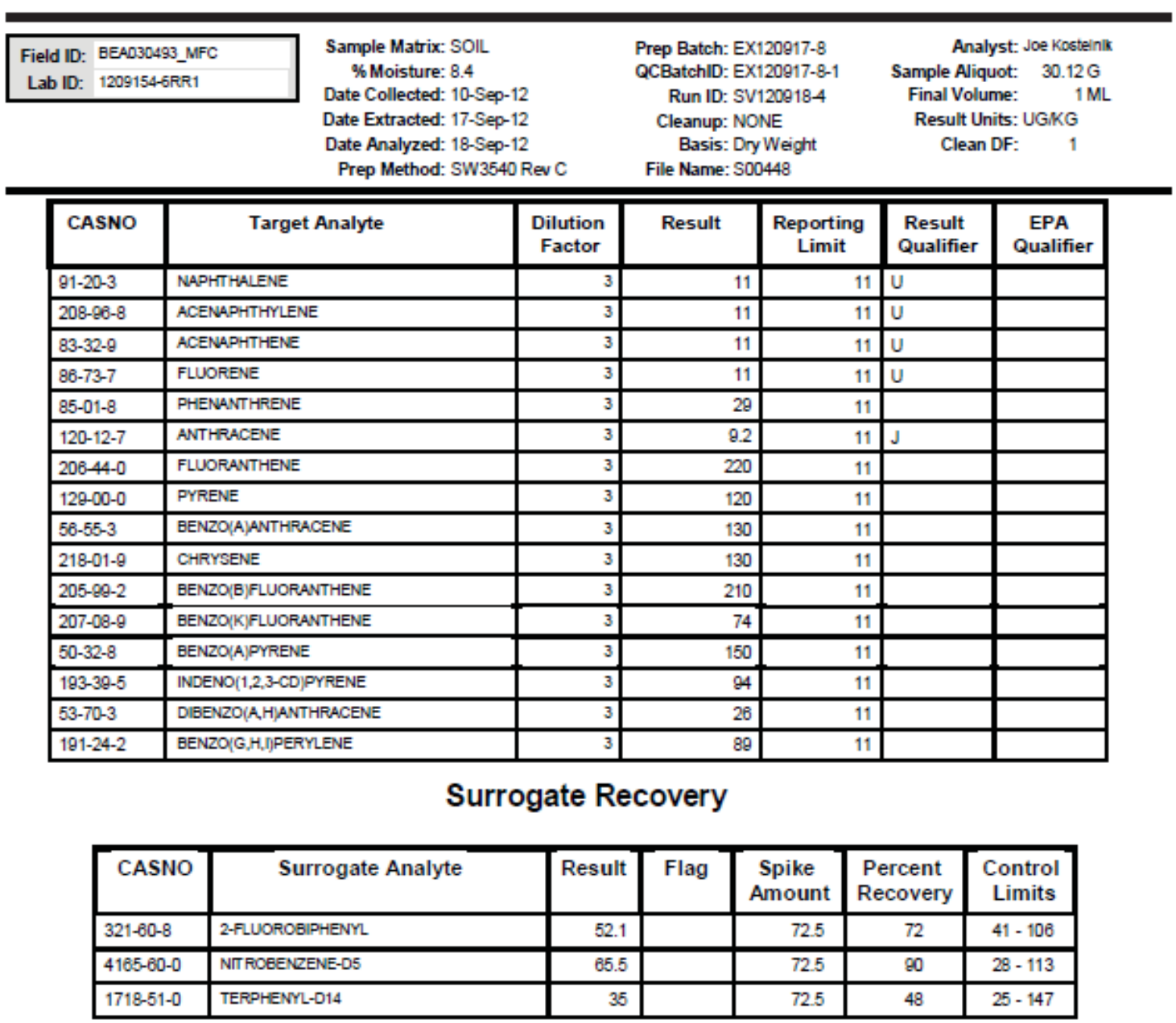

Data Package ID: SV1209154-1

$\begin{array}{cc}\text { Date Printed: Wednesday, September 19, } 2012 & \text { ALS Environmental -- FC } \\ \text { LMS Version: } 6.611\end{array}$

19 of 33 


\section{A}

\section{Supporting QA/QC Data}

20 of 33 


\title{
Surrogate Summary for GC/MS Semi-volatiles
}

\author{
Method SW8270SIMPAHD
}

Lab Name: ALS Environmental - FC

Work Order Number: 1209154

Client Name: Battelle Energy Alliance

ClientProject ID: MFC Biodiesel Tank Removal ,TOS-A1175 BEA030488

\begin{tabular}{|c|c|c|c|}
\hline \multirow{2}{*}{$\begin{array}{l}\text { PrepBatchID: EX120917-8 } \\
\text { QC Batch ID: EX120917-8-1 }\end{array}$} & Surrogate Compound & \multicolumn{2}{|c|}{ Control Limits } \\
\hline & 2,4,6-Tribromophenol & & \\
\hline \multirow[t]{5}{*}{ Date Extracted: 9/17/2012 } & 2-Fluorobiphenyl & 41 & 106 \\
\hline & 2-Fluorophend & & \\
\hline & Nitrobenzene-d5 & 28 & 113 \\
\hline & Phenol-d5 & & \\
\hline & Terphenyi-d14 & 25 & 147 \\
\hline
\end{tabular}

\begin{tabular}{|c|c|c|c|c|c|c|c|c|c|}
\hline Lab ID & Client Sample ID & $\begin{array}{c}\text { Date } \\
\text { Collected }\end{array}$ & $\begin{array}{c}\text { Date } \\
\text { Received }\end{array}$ & $\begin{array}{l}246 T B \% \\
\text { Recovery }\end{array}$ & $\begin{array}{l}\text { 2FBP \% } \\
\text { Recovery }\end{array}$ & $\begin{array}{c}\text { 2FP \% } \\
\text { Recovery }\end{array}$ & $\begin{array}{c}\text { ND5 \% } \\
\text { Recovery }\end{array}$ & $\begin{array}{c}\text { PD5 \% } \\
\text { Ricovery }\end{array}$ & $\begin{array}{l}\text { TD14\% } \% \\
\text { Recovery }\end{array}$ \\
\hline EX120917-8MB & $x 0000000 x$ & NA & $\mathrm{x} x 000000$ & & 83 & & 84 & & 95 \\
\hline EX120917-8LCS & $x 000 x x 00 x$ & NA & $x x 000 x x$ & & 78 & & 87 & & 85 \\
\hline EX120917-8LCSD & $x 000 X X 0 X X$ & NA & 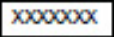 & & 81 & & 88 & & 87 \\
\hline $1209154-3$ & BEA030490_MFC & $9 / 10 / 2012$ & $9 / 12 / 2012$ & & 78 & & 90 & & 87 \\
\hline $1209154-2$ & BEA030489_MFC & $9 / 10 / 2012$ & $9 / 12 / 2012$ & & 74 & & 84 & & 94 \\
\hline 1209154-2MS & BEA030489_MFC & $9 / 10 / 2012$ & $9 / 12 / 2012$ & & 75 & & 87 & & 87 \\
\hline 1209154-2MSD & BEA030489_MFC & $9 / 10 / 2012$ & $9 / 12 / 2012$ & & 70 & & 85 & & 74 \\
\hline $1209154-7$ & BEA030494_MFC & $9 / 10 / 2012$ & $9 / 12 / 2012$ & & 76 & & 87 & & 76 \\
\hline $1209154-11$ & BEA030498_MFC & $9 / 10 / 2012$ & $9 / 12 / 2012$ & & 73 & & 83 & & 63 \\
\hline $1209154-6$ & BEA030493_MFC & 9/10/2012 & 9/12/2012 & & 76 & & 96 & & 85 \\
\hline $1209154-10$ & BEA030497_MFC & $9 / 10 / 2012$ & 9/12/2012 & & 82 & & 105 & & 75 \\
\hline 1209154-6RR1 & BEA030493_MFC & 9/10/2012 & $9 / 12 / 2012$ & & 72 & & 90 & & 48 \\
\hline
\end{tabular}

\begin{tabular}{ccc}
\hline Date Printed: Wednesday, September 19, 2012 & ALS Environmental - FC \\
Lams verion: 6.611 & Page 1 of 1 \\
Shaded values exceed established control limits. & & 21 of 33
\end{tabular}




\section{GC/MS Semi-volatiles}

Method SW8270SIMPAHD

Laboratory Control Sample and Laboratory Control Sample Duplicate

Lab Name: ALS Environmental - FC

Work Order Number: 1209154

Client Name: Battele Energy Alliance

ClientProject ID: MFC Biodiesel Tank Removal ,TOS-A1175 BEA030488

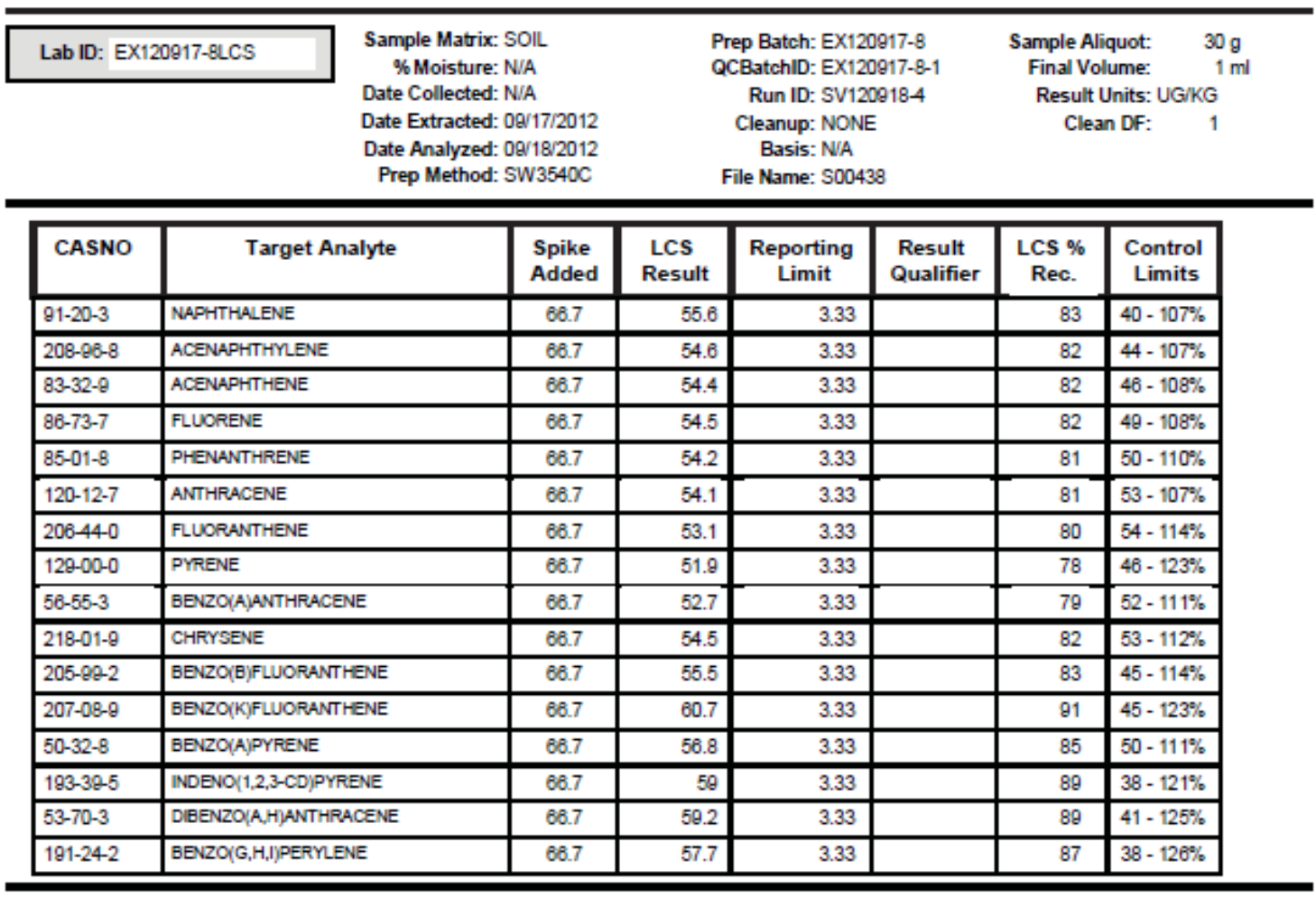

Data Package ID: SV1209154-1

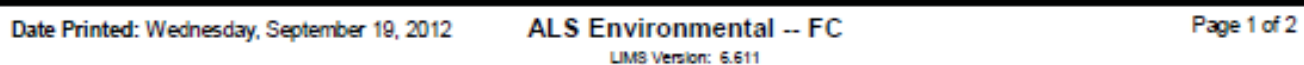

22 of 33 


\title{
GC/MS Semi-volatiles
}

\author{
Method SW8270SIMPAHD
}

Laboratory Control Sample and Laboratory Control Sample Duplicate

Lab Name: ALS Environmental - FC

Work Order Number: 1209154

Client Name: Battele Energy Alliance

ClientProject ID: MFC Biodiesel Tank Removal ,TOS-A1175 BEA030488

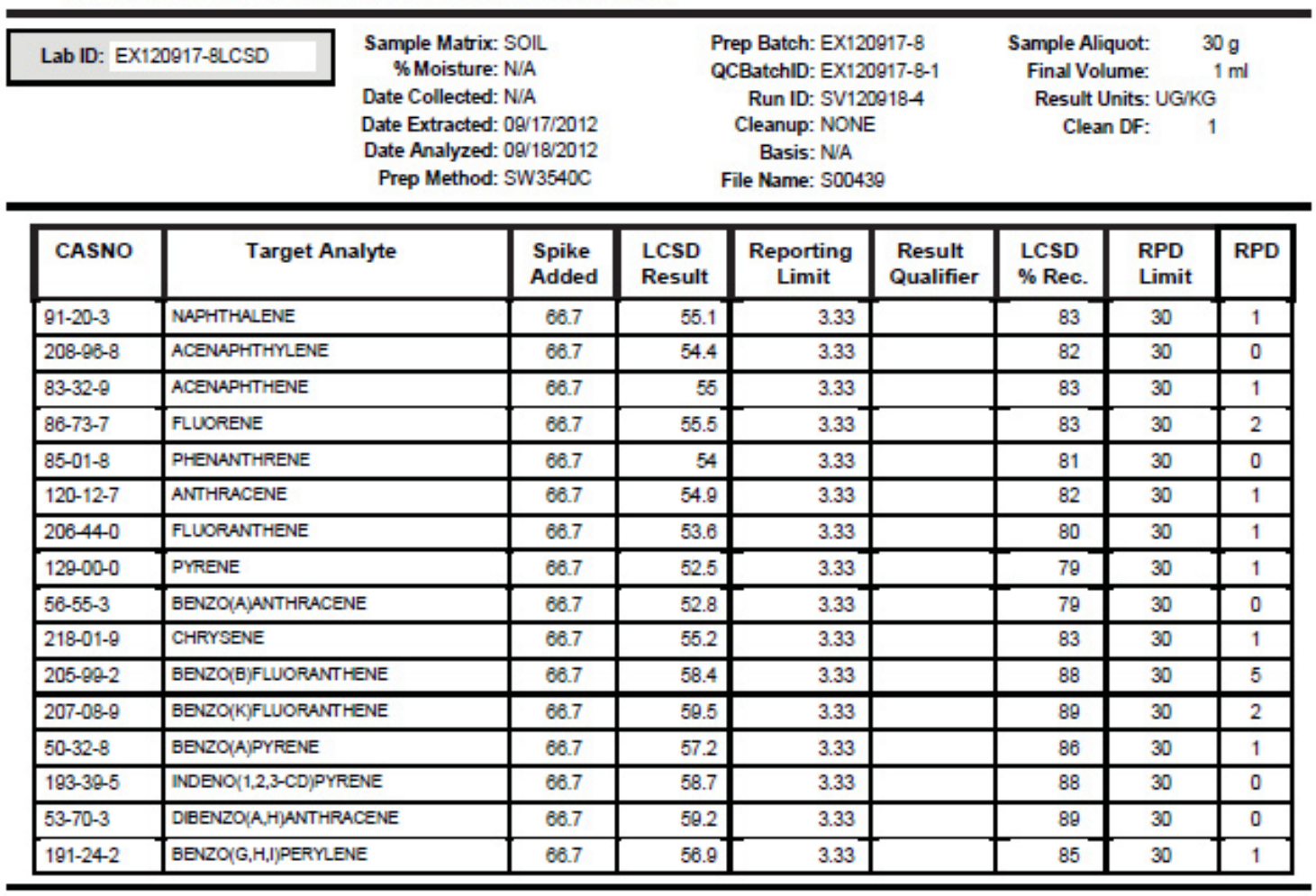

Surrogate Recovery LCS/LCSD

\begin{tabular}{|l|l|r|r|r|r|c|c|}
\hline CASNO & \multicolumn{1}{|c|}{ Target Analyte } & $\begin{array}{c}\text { Spike } \\
\text { Added }\end{array}$ & $\begin{array}{c}\text { LCS \% } \\
\text { Rec. }\end{array}$ & $\begin{array}{c}\text { LCS } \\
\text { Flag }\end{array}$ & $\begin{array}{c}\text { LCSD } \\
\% \text { Rec. }\end{array}$ & $\begin{array}{c}\text { LCSD } \\
\text { Flag }\end{array}$ & $\begin{array}{c}\text { Control } \\
\text { Limits }\end{array}$ \\
\hline $321-60-8$ & 2-FLUOROBIPHENYL & 66.7 & 78 & & 81 & & $41-106$ \\
\hline $4165-60-0$ & NTROBENZENE-D5 & 68.7 & 87 & & 88 & & $28-113$ \\
\hline $1718-51-0$ & TERPHENYL-D14 & 66.7 & 85 & & 87 & & $25-147$ \\
\hline
\end{tabular}

Data Package ID: SV1209154-1

Date Printed: Wednesday, September 19, 2012

ALS Environmental - FC

Page 2 of 2

uMs version: 6.611

23 of 33 
Matrix Spike And Matrix Spike Duplicate

Lab Name: ALS Environmental - FC

Work Order Number: 1209154

Client Name: Battelle Energy Alliance

ClientProject ID: MFC Biodiesel Tank Removal, TOS-A1175 BEA030488

\begin{tabular}{|c|c|c|c|}
\hline Field ID: EEAD30489_MFC & $\begin{array}{c}\text { Sample Matrix: SOIL } \\
\text { \% Moisture: } 11.6\end{array}$ & $\begin{array}{l}\text { Prep Batch: EX120917-8 } \\
\text { QCBatchID: EX120917-8-1 }\end{array}$ & $\begin{array}{rr}\text { Sample Aliquot: } & 30.48 \mathrm{~g} \\
\text { Final Volume: } & 1 \mathrm{ml}\end{array}$ \\
\hline LabID: 1209154-2MS & Date Collected: $10-S e p-12$ & Run ID: SV120018-4 & Result Units: UG/KG \\
\hline & Date Extracted: $17-S e p-12$ & Cleanup: NONE & File Name: 500442 \\
\hline & $\begin{array}{l}\text { Date Analyzed: } 18-S e p-12 \\
\text { Prep Method: SW3540 Rev C }\end{array}$ & Basis: DryWeight & \\
\hline
\end{tabular}

\begin{tabular}{|c|c|c|c|c|c|c|c|c|c|}
\hline CASNO & Target Analyte & $\begin{array}{r}\text { Sample } \\
\text { Result }\end{array}$ & $\begin{array}{r}\text { Samp } \\
\text { Qual }\end{array}$ & $\begin{array}{c}\text { MS } \\
\text { Result }\end{array}$ & $\begin{array}{l}\text { MS } \\
\text { Qual }\end{array}$ & $\begin{array}{l}\text { Reporting } \\
\text { Limit }\end{array}$ & $\begin{array}{l}\text { Spike } \\
\text { Added }\end{array}$ & $\begin{array}{l}\text { MS \% } \\
\text { Rec. }\end{array}$ & $\begin{array}{l}\text { Control } \\
\text { Limits }\end{array}$ \\
\hline $91-20-3$ & NAPHTHALENE & 2 & $\mathrm{~J}$ & 58.6 & & 3.71 & 74.3 & 76 & $40-107 \%$ \\
\hline $208-96-8$ & ACENAPHTHYLENE & 2.3 & $\mathrm{~J}$ & 64.3 & & 3.71 & 74.3 & 83 & $44-107 \%$ \\
\hline $83-32-8$ & ACENAPHTHENE & 3.7 & $\bar{U}$ & 55.9 & & 3.71 & 74.3 & 75 & $46-108 \%$ \\
\hline $86-73-7$ & FLUORENE & 3.7 & u & 68.6 & & 3.71 & 74.3 & 80 & $48-108 \%$ \\
\hline $85-01-8$ & PHENANTHRENE & 1.6 & $\mathrm{~J}$ & 65.5 & & 3.71 & 74.3 & 88 & $50-110 \%$ \\
\hline $120-12-7$ & ANTHRACENE & 1.7 & $\mathrm{~J}$ & 63.2 & & 3.71 & 74.3 & 83 & $53-107 \%$ \\
\hline $206-44-0$ & FLUORANTHENE & 1.4 & $\mathrm{~J}$ & 75.7 & & 3.71 & 74.3 & 100 & $54-114 \%$ \\
\hline $129-00-0$ & PYRENE & 2.2 & $\mathrm{~J}$ & 75.3 & & 3.71 & 74.3 & 89 & $46-123 \%$ \\
\hline $56-55-3$ & BENZO(A)ANTHRACENE & 1.3 & $\mathrm{~J}$ & 73.4 & & 3.71 & 74.3 & 97 & $52-111 \%$ \\
\hline $218-01-\theta$ & CHRYSENE & 3.7 & u & 75.2 & & 3.71 & 74.3 & 101 & $53-112 \%$ \\
\hline $205-99-2$ & BENZO(B)FLUORANTHENE & 2.7 & $\mathrm{~J}$ & 83.4 & & 3.71 & 74.3 & 109 & $45-114 \%$ \\
\hline $207-08-8$ & BENZO(K)FLUORANTHENE & 3.7 & $\mathrm{u}$ & 72.6 & & 3.71 & 74.3 & 88 & $45-123 \%$ \\
\hline $50-32-8$ & BENZO(A)PYRENE & 2.3 & $\mathrm{~J}$ & 80.6 & & 3.71 & 74.3 & 108 & $50-111 \%$ \\
\hline $193-39-5$ & INDENO $(1,2,3$-CD $)$ PYRENE & 2.5 & $\mathrm{~J}$ & 79.9 & & 3.71 & 74.3 & 104 & $38-121 \%$ \\
\hline $53-70-3$ & DIEENZO(A,H)ANTHRACENE & 3.7 & U & 76.4 & & 3.71 & 74.3 & 103 & $41-125 \%$ \\
\hline $191-24-2$ & BENZO(G,H,I)PERYLENE & 4.4 & & 74.3 & & 3.71 & 74.3 & 24 & $38-126 \%$ \\
\hline
\end{tabular}

Data Package ID: SV1209154-1

Date Printed: Wednesday, September 19, 2012

ALS Environmental -- FC

LMS Version: 6.511
Page 1 of 2

24 of 33 


\title{
GC/MS Semi-volatiles
}

\author{
Method SW8270SIMPAHD
}

Matrix Spike And Matrix Spike Duplicate

Lab Name: ALS Environmental - FC

Work Order Number: 1209154

Client Name: Battelle Energy Alliance

ClientProject ID: MFC Biodiesel Tank Removal ,TOS-A1175 BEA030488

\begin{tabular}{|c|c|c|c|}
\hline $\begin{array}{r}\text { Field ID: } 8 E A 030489 \text { MFC } \\
\text { LabID: } 1209154-2 M S D\end{array}$ & $\begin{array}{l}\text { Sample Matrix: SOIL } \\
\quad \text { \% Moisture: } 11.6 \\
\text { Date Collected: } 10-\text { Sep-12 }\end{array}$ & $\begin{array}{l}\text { Prep Batch: EX120917-8 } \\
\text { QCBatchID: EX120917-8-1 } \\
\text { Run ID: SV120918-4 }\end{array}$ & $\begin{array}{c}\text { Sample Aliquot: } \quad 30.11 \mathrm{~g} \\
\text { Final Volume: } 1 \mathrm{ml} \\
\text { Result Units: UG/KG }\end{array}$ \\
\hline & $\begin{array}{l}\text { Date Extracted: } 17-\text { Sep-12 } \\
\text { Date Analyzed: } 18-\text { Sep-12 } \\
\text { Prep Method: SW3540 Rev C }\end{array}$ & $\begin{array}{l}\text { Cleanup: NONE } \\
\text { Basis: DryWeight }\end{array}$ & File Name: 500443 \\
\hline
\end{tabular}

\begin{tabular}{|c|c|c|c|c|c|c|c|c|}
\hline CASNO & Target Analyte & $\begin{array}{l}\text { MSD } \\
\text { Result }\end{array}$ & $\begin{array}{l}\text { MSD } \\
\text { Qual }\end{array}$ & $\begin{array}{l}\text { Spike } \\
\text { Added }\end{array}$ & $\begin{array}{l}\text { MSD \% } \\
\text { Rec. }\end{array}$ & $\begin{array}{l}\text { Reporting } \\
\text { Limit }\end{array}$ & $\begin{array}{l}\text { RPD } \\
\text { Limit }\end{array}$ & RPD \\
\hline $91-20-3$ & NAPHTHALENE & 56.7 & & 75.2 & 73 & 3.78 & 30 & 3 \\
\hline $208-96-8$ & ACENAPHTHYLENE & 57.6 & & 75.2 & 74 & 3.76 & 30 & 11 \\
\hline $83-32-8$ & ACENAPHTHENE & 51.6 & & 75.2 & 69 & 3.78 & 30 & 8 \\
\hline $86-73-7$ & FLUORENE & 60.8 & & 75.2 & 81 & 3.76 & 30 & 9 \\
\hline $85-01-8$ & PHENANTHRENE & 60.7 & & 75.2 & 79 & 3.76 & 30 & 8 \\
\hline $120-12-7$ & ANTHRACENE & 61.2 & & 75.2 & 79 & 3.78 & 30 & 3 \\
\hline $206-44-0$ & FLUORANTHENE & 62.4 & & 75.2 & 81 & $3.76^{-}$ & 30 & 19 \\
\hline $129-00-0$ & PYRENE & 55.1 & + & 75.2 & 70 & 3.76 & 30 & 31 \\
\hline $56-55-3$ & BENZO(A)ANTHRACENE & 60.1 & & 75.2 & 78 & 3.78 & 30 & 20 \\
\hline $218-01-\theta$ & CHRYSENE & 60.3 & & 75.2 & 80 & 3.76 & 30 & 22 \\
\hline $205-99-2$ & BENZO(B)FLUORANTHENE & 61 & + & 75.2 & 78 & 3.78 & 30 & 31 \\
\hline $207-08-9$ & BENZO(K)FLUORANTHENE & 61.7 & & 75.2 & 82 & 3.78 & 30 & 16 \\
\hline $50-32-8$ & BENZO(A)PYFENE & 64.5 & & 75.2 & 83 & 3.76 & 30 & 22 \\
\hline $193-39-5$ & INDENO(1,2,3-CD)PYRENE & 68.3 & & 75.2 & 85 & 3.78 & 30 & 19 \\
\hline $53-70-3$ & DIEENZO(A,H)ANTHRACENE & 70 & & 75.2 & 93 & 3.76 & 30 & 8 \\
\hline $191-24-2$ & BENZO(G,H,I)PERY_ENE & 57.7 & & 75.2 & 71 & 3.78 & 30 & 25 \\
\hline
\end{tabular}

Surrogate Recovery MS/MSD

\begin{tabular}{|l|l|r|r|r|r|r|c|}
\hline CASNO & \multicolumn{1}{|c|}{ Target Analyte } & $\begin{array}{c}\text { Spike } \\
\text { Added }\end{array}$ & $\begin{array}{c}\text { MS \% } \\
\text { Rec. }\end{array}$ & $\begin{array}{c}\text { MS } \\
\text { Flag }\end{array}$ & $\begin{array}{c}\text { MSD \% } \\
\text { Rec. }\end{array}$ & $\begin{array}{c}\text { MSD } \\
\text { Flag }\end{array}$ & $\begin{array}{c}\text { Control } \\
\text { Limits }\end{array}$ \\
\hline $321-60-8$ & 2-FLUOROEIPHENY & 74.3 & 75 & & 70 & & $41-106$ \\
\hline $4165-60-0$ & NITROEENZENE-D5 & 74.3 & 87 & & 85 & & $28-113$ \\
\hline $1718-51-0$ & TERPHENYL-D14 & 74.3 & 87 & & 74 & & $25-147$ \\
\hline
\end{tabular}

Data Package ID: SV1209154-1

$\begin{array}{lcc}\text { Date Printed: Wechesday, September 19, } 2012 & \begin{array}{c}\text { ALS Environmental -- FC } \\ \text { Lms version: 6.511 }\end{array} & \text { Page } 2 \text { of } 2\end{array}$




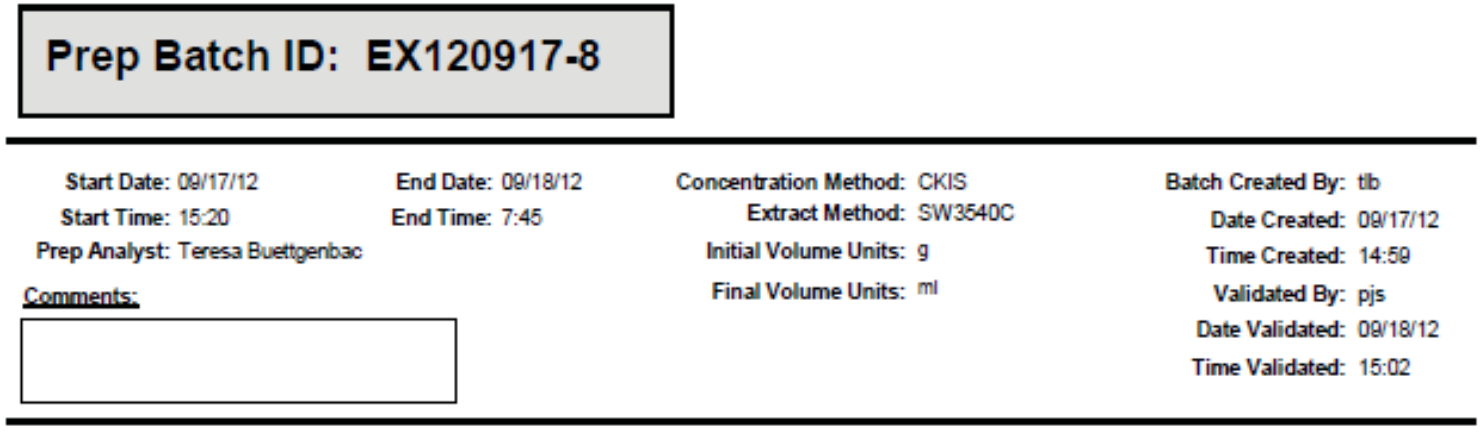

QC Batch ID: EX120917-8-1

\begin{tabular}{|c|c|c|c|c|c|c|c|c|c|}
\hline Lab ID & $\begin{array}{l}\text { QC } \\
\text { Type }\end{array}$ & Field ID & Matrix & $\begin{array}{c}\text { Date } \\
\text { Collected }\end{array}$ & $\begin{array}{l}\text { Initial } \\
\text { WtVol }\end{array}$ & $\begin{array}{l}\text { Final } \\
\text { WtVol }\end{array}$ & $\begin{array}{l}\text { Cleanup } \\
\text { Method }\end{array}$ & $\begin{array}{c}\text { Cleanup } \\
\text { DF }\end{array}$ & $\begin{array}{l}\text { Order } \\
\text { Number }\end{array}$ \\
\hline EX120917-8 & $\mathrm{MB}$ & $\mathrm{XX000XX}$ & SOIL & $\overline{X X C D C X}$ & 30 & 1 & NONE & 1 & 1209154 \\
\hline Ex1 $\overline{20917-8}$ & LCS & $x x 000 x$ & SOIL & $\overline{X X C D C X}$ & 30 & 1 & NONE & 1 & 1209154 \\
\hline EX1 $\overline{20917-8}$ & $\overline{L C S D}$ & $\overline{x x 000 x}$ & $\overline{\text { SOIL }}$ & $\overline{X X X X C X X}$ & 30 & 1 & NONE & 1 & 1209154 \\
\hline$1 2 0 \longdiv { 9 1 5 4 - 2 }$ & $\overline{M S}$ & BEAD30489_MFC & $\overline{\text { SOIL }}$ & $9 / 10 / 2012$ & 30.48 & 1 & NONE & 1 & 1209154 \\
\hline $120 \overline{9154-2}$ & $\overline{M S D}$ & BEAD30489_MFC & SOIL & $9 / 10 / 2012$ & 30.11 & 1 & NONE & 1 & 1200154 \\
\hline $120 \overline{9154-10}$ & SMP & BEAD30497_MFC & $\overline{\text { SOIL }}$ & $9 / 10 / 2012$ & 30.63 & 1 & NONE & 1 & 1209154 \\
\hline $120 \overline{9154-11}$ & SMP & BEAD30498_MFC & $\overline{\text { SOIL }}$ & $9 / 10 / 2012$ & 30.42 & 1 & NONE & 1 & 1209154 \\
\hline $120 \overline{9154-2}$ & SMP & BEAD30489_MFC & SOIL & $9 / 10 / 2012$ & 30.52 & 1 & NONE & 1 & 1200154 \\
\hline $120 \overline{9154-3}$ & SMP & BEAD30490_MFC & SOIL & $9 / 10 / 2012$ & 30.63 & 1 & NONE & 1 & 1209154 \\
\hline $120 \overline{9154-6}$ & SMP & BEAD30493_MFC & SOIL & $9 / 10 / 2012$ & 30.12 & 1 & NONE & 1 & 1209154 \\
\hline $1209 \overline{154-7}$ & SMP & BEA030494_MFC & SOIL & $9 / 10 / 2012$ & 30.86 & 1 & NONE & 1 & 1209154 \\
\hline
\end{tabular}

In generating this benchsheet, prep analyst states that al aspects of sample preparation as set forth in the appropriate SOP's (inciucling KudemaDanish temperatures, proper flow settings on the N-evap, and final volumes) were properily achered to (unless otherwise noted herein).

\section{ec Trues}

\begin{tabular}{|l|l|l|l|l|}
\hline CAR & Carrier reference sample & DUP & Laboratory Duplicate \\
\hline LCS & Laboratory Control Sample & LCSD & Laboratory Control Sample Duplicat \\
\hline ME & Method Blank & MS & Laboratory Matrix Splke \\
\hline MSD & Laboratory Matrix Splike Duplicate & REP & Sample replicate \\
\hline RVS & Reporting Level Verfifcation Standar & SMP & Fleld Sample \\
\hline SYS & Sample Yeld Splke & \\
\hline
\end{tabular}




\section{B}

Semi-Volatile Organic GC/MS Tuning And Mass

Calibration--Decafluorotriphenylphosphine (DFTPP)

Lab Name: ALS Environmental - FC Work Order Number: 1209154

Client Name: Battelle Energy Alliance

ClientProject ID: BEA030488MFC Biodiesel Tank Removal ,TOS-A1175
DFTPP Injection Date: $\quad$ 8/18/2012 DFTPP Injection Time: $\quad$ 11:43

Instrument ID: HPSV4

Reported on: Wednesday, September 19, 2012

FileID: 500428

\begin{tabular}{|l|l|c|}
\hline mie & $\begin{array}{c}\text { lon Abundance Criteria } \\
\text { SW8270SIMPAHD }\end{array}$ & $\begin{array}{c}\text { \%Relative } \\
\text { Abundance }\end{array}$ \\
\hline 51 & $30.0-60.0$ percent of mass 198 & 45.1 \\
\hline 68 & Less than 2.0 percent of mass 69 & 0 \\
\hline 69 & Mass 69 relative abundance of mass 198 & 46.9 \\
\hline 70 & Less than 2.0 percent of mass 69 & 0.5 \\
\hline 127 & $40.0-60.0$ percent of mass 198 & 51.5 \\
\hline 197 & Less than 1.0 percent of mass 198 & 0 \\
\hline 198 & Base peak. 100 percent of relative abundance & 100 \\
\hline 199 & $5.0-9.0$ percent of mass 198 & 6.7 \\
\hline 275 & $10.0-30.0$ percent of mass 198 & 25.8 \\
\hline 365 & Greater than 1.00 percent of mass 188 & 2.8 \\
\hline 441 & Present, but less than mass 443 (percent of 443 ) & 82.4 \\
\hline 442 & Greater than 40.0 percent of mass 188 & 82.6 \\
\hline 443 & 17.0 - 23.0 percent of mass 442 & 19.3 \\
\hline
\end{tabular}

THIS TUNE APPLIES TO THE FOLLOWING SAMPLES, MS/MSD, BLANKS, AND STANDARDS:

\begin{tabular}{|c|c|c|c|c|c|}
\hline Client Sample ID & Lab Sample ID & Lab File ID & $\begin{array}{c}\text { Date } \\
\text { Analyzed }\end{array}$ & $\begin{array}{c}\text { Time } \\
\text { Analyzed }\end{array}$ & QC BatchID \\
\hline$x 000000 x$ & ICALSVSTD0500CSTD & 500429 & $9 / 18 / 2012$ & 11.54 & SV120918-4 \\
\hline $1000000 X$ & ICALSVSTD0050CSTD & 500430 & $9 / 18 / 2012$ & $12: 13$ & SV120918-4 \\
\hline $2000000 x$ & ICALSVSTD0100CSTD & $S 00431$ & $9 / 18 / 2012$ & $12: 32$ & SV120918-4 \\
\hline$x 000000 x$ & ICALSVSTD0200CSTD & $\mathrm{S} 00432$ & $9 / 18 / 2012$ & $12-52$ & SV120918-4 \\
\hline$x 000000 x$ & ICALSVSTD1000CSTD & $\mathrm{S} 00433$ & $9 / 18 / 2012$ & $13: 11$ & SV120918-4 \\
\hline $1000000 X$ & ICALSVSTD2000CSTD & 500434 & $9 / 18 / 2012$ & $13: 30$ & SV120918-4 \\
\hline $2000000 x$ & ICALSVSTD5000CSTD & 500435 & $9 / 18 / 2012$ & $13: 49$ & SV120918-4 \\
\hline $1000000 x$ & ICVSVSTD2000ICV & 500436 & $9 / 18 / 2012$ & $14: 08$ & SV120918-4 \\
\hline $2000000 x$ & EX120917-8MB & 500437 & $9 / 18 / 2012$ & $14: 38$ & EX120917-8-1 \\
\hline $100000 C X$ & EX120917-8LCS & $\mathrm{S} 00438$ & $9 / 18 / 2012$ & 14.57 & EX120917-8-1 \\
\hline $2000000 x$ & EX120917-8LCSD & 500439 & $9 / 18 / 2012$ & $15: 17$ & EX120917-8-1 \\
\hline EEAD30490_MFC & $1209154-3$ & $\mathrm{~S} 00440$ & $9 / 18 / 2012$ & $15: 41$ & EX120917-8-1 \\
\hline BEAD30489_MFC & $1209154-2$ & S00441 & 9/18/2012 & $16: 01$ & EX120917-8-1 \\
\hline
\end{tabular}

Data Package ID: SV1209154-1

\footnotetext{
Date Printed: Wednesday, September 19, $2012 \quad$ ALS Environmental - FC
}

Page 1 of 2

27 of 33 
5B

Semi-Volatile Organic GC/MS Tuning And Mass

Calibration--Decafluorotriphenylphosphine (DFTPP)

Lab Name: ALS Environmental - FC

Work Order Number: 1209154

Client Name: Battelle Energy Alliance

ClientProject ID: BEA030488MFC Biodiesel Tank Removal ,TOS-A1175

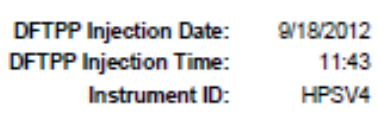

Reported on: Wednesday, September 19, 2012

FilelD: 500428

\begin{tabular}{|c|l|c|}
\hline mie & $\begin{array}{c}\text { lon Abundance Criteria } \\
\text { SW8270SIMPAHD }\end{array}$ & $\begin{array}{c}\text { \% Relative } \\
\text { Abundance }\end{array}$ \\
\hline 51 & $30.0-60.0$ percent of mass 198 & 45.1 \\
\hline 68 & Less than 2.0 percent of mass 69 & 0 \\
\hline 69 & Mass 69 relative abundance of mass 198 & 46.8 \\
\hline 70 & Less than 2.0 percent of mass 69 & 0.5 \\
\hline 127 & $40.0-60.0$ percent of mass 198 & 51.5 \\
\hline 197 & Less than 1.0 percent of mass 198 & 0 \\
\hline 198 & Base peak, 100 percent of relative abundance & 100 \\
\hline 199 & $5.0-9.0$ percent of mass 198 & 6.7 \\
\hline 275 & $10.0-30.0$ percent of mass 198 & 25.8 \\
\hline 365 & Greater than 1.00 percent of mass 188 & 2.8 \\
\hline 441 & Present, but less than mass 443 (percent of 443 ) & 82.4 \\
\hline 442 & Greater than 40.0 percent of mass 188 & 82.6 \\
\hline 443 & $17.0-23.0$ percent of mass 442 & 19.3 \\
\hline
\end{tabular}

THIS TUNE APPLIES TO THE FOLLOWING SAMPLES, MS/MSD, BLANKS, AND STANDARDS:

\begin{tabular}{|c|c|c|c|c|c|}
\hline Client Sample ID & Lab Sample ID & Lab File ID & $\begin{array}{c}\text { Date } \\
\text { Analyzed }\end{array}$ & $\begin{array}{c}\text { Time } \\
\text { Analyzed }\end{array}$ & QC BatchID \\
\hline BEAD30489_MFC & $1209154-2 M S$ & S00442 & 9/18/2012 & $16: 20$ & EX120917-8-1 \\
\hline BEAD30489_MFC & $1209154-2 M S D$ & $\mathrm{~S} 00443$ & $9 / 18 / 2012$ & $16: 39$ & EX120917-8-1 \\
\hline BEAD30494_MFC & $1209154-7$ & 500444 & 9/18/2012 & 16.58 & EX120917-8-1 \\
\hline BEAD30498_MFC & $1209154-11$ & $S 00445$ & 9/18/2012 & $17: 17$ & EX120917-8-1 \\
\hline BEAD30493_MFC & $1209154-6$ & $S 00448$ & 9/18/2012 & $17: 36$ & EX120917-8-1 \\
\hline BEAD30497_MFC & $1209154-10$ & $S 00447$ & $9 / 18 / 2012$ & $17-56$ & EX120917-8-1 \\
\hline BEAD30493_MFC & 1209154-6RR1 & S00448 & 8/18/2012 & $18: 15$ & EX120917-8-1 \\
\hline
\end{tabular}

ALS Environmental - FC LaMs version: 6.611
Page 2 of 2

28 of 33 


\begin{tabular}{|c|c|c|c|c|c|c|c|c|c|c|c|c|}
\hline \multirow{3}{*}{ 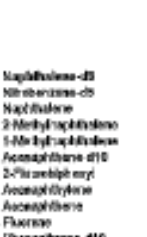 } & 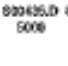 & & Stoting & 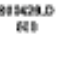 & ${ }_{200}^{2000}$ & 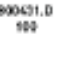 & $\begin{array}{lll}0460 \\
50\end{array}$ & Anewere & $\operatorname{sen}$ & \multicolumn{2}{|c|}{ 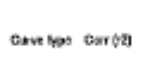 } & 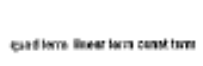 \\
\hline & 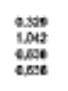 & 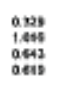 & 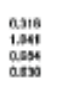 & 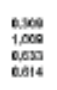 & 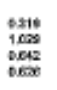 & 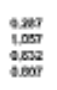 & 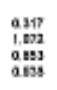 & 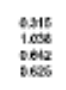 & 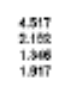 & 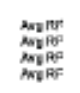 & $\begin{array}{l}m \\
m \\
w \\
m\end{array}$ & \\
\hline & 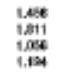 & 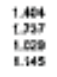 & 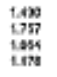 & $\begin{array}{l}1,360 \\
1,700 \\
1.90 \\
1.900\end{array}$ & 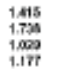 & 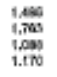 & 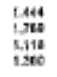 & 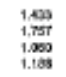 & $\begin{array}{l}2239 \\
1,39 \\
\text { sam } \\
4,39 m\end{array}$ & 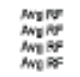 & 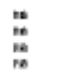 & \\
\hline 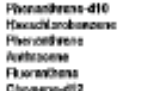 & 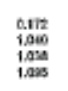 & 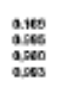 & 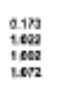 & 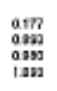 & 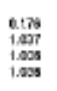 & 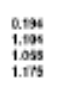 & 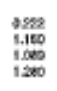 & 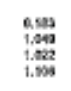 & 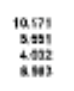 & 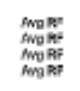 & 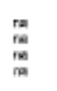 & \\
\hline 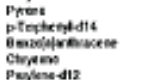 & 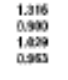 & 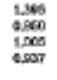 & 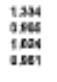 & 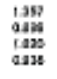 & 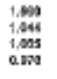 & 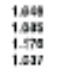 & 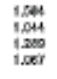 & 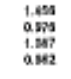 & 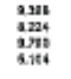 & 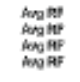 & 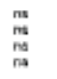 & \\
\hline 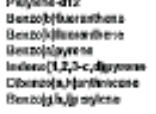 & 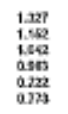 & 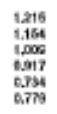 & 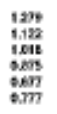 & 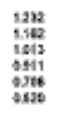 & 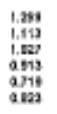 & 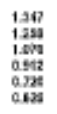 & 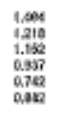 & 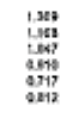 & 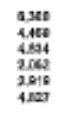 & 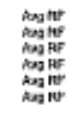 & $\begin{array}{l}m \\
m \\
m \\
m \\
n \\
m \\
m\end{array}$ & \\
\hline & & & & & & & & nhae: & 4.500 & & & \\
\hline
\end{tabular}

$$
q_{1}+1,12
$$

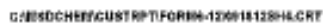




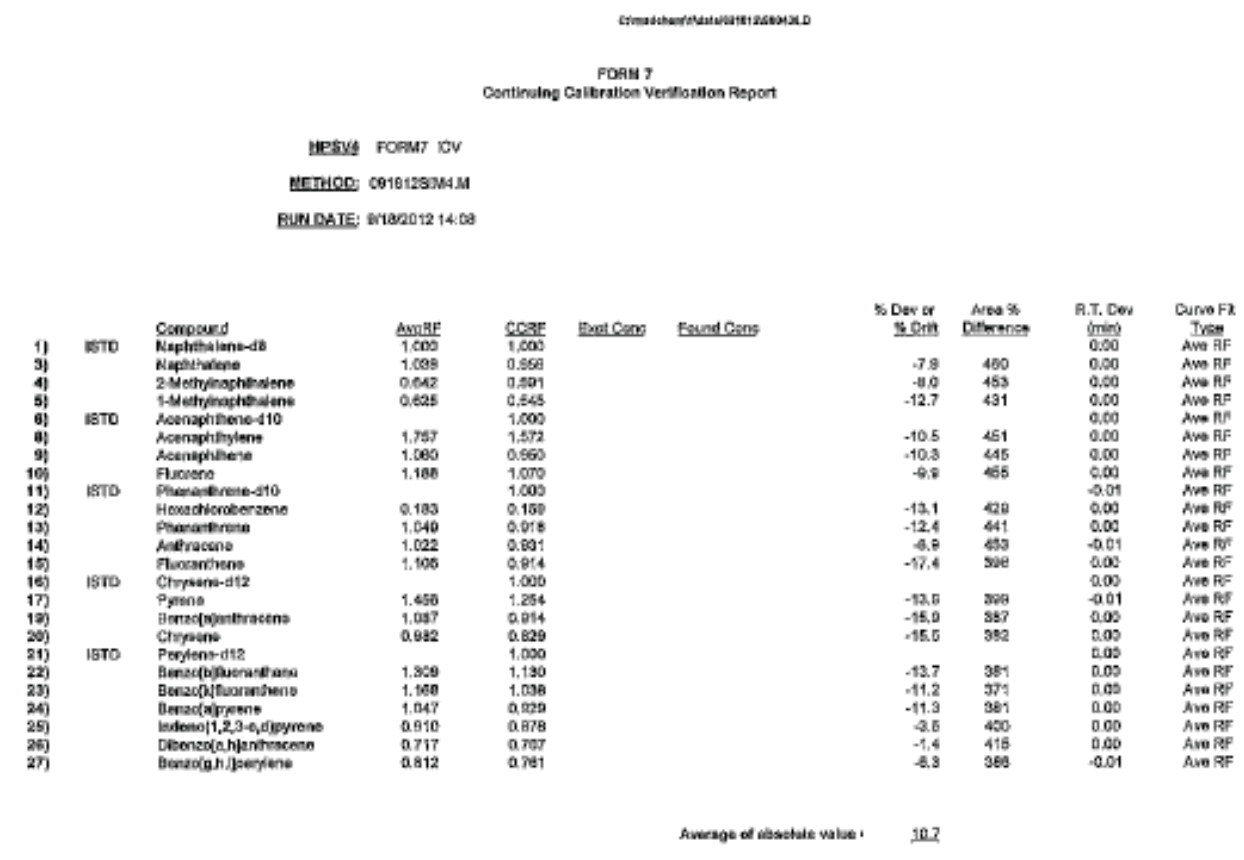

$\frac{9 \pi}{9-18}$

Hon:1

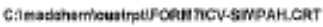

30 of 33

varizzen 
8B

Semi-Volatile Internal Standard Area Summary

Lab Name: ALS Environmental - FC

Date Analyzed: $\quad 9 / 18 / 2012$

Work Order Number: 1209154

Time Analyzed:

Client Name: Battelle Energy Alliance

Reported on: Wednesday, September 19, 2012

ClientProject ID: BEA030488 MFC Biodiesel Tank Remova ,TOS-A1175

Instrument ID: HPSV4

Lab File ID: $\quad$ S00429

\begin{tabular}{|c|c|c|c|c|c|c|c|c|c|c|c|c|}
\hline & \multicolumn{2}{|c|}{ IS1 } & \multicolumn{2}{|l|}{ IS2 } & \multicolumn{2}{|l|}{ IS3 } & \multicolumn{2}{|l|}{ IS4 } & \multicolumn{2}{|c|}{ IS5 } & \multicolumn{2}{|c|}{ IS6 } \\
\hline & Area & RT & Area & RT & Area & RT & Area & RT & Area & RT & Area & RT \\
\hline 12 Hour STD & & & 683711 & 5.48 & 320471 & 7 & 455222 & 8.29 & 366097 & 10.61 & 238070 & 12.28 \\
\hline Upper Limit & & & 1327422 & 5.96 & 640942 & 7.5 & 910444 & 8.79 & 732184 & 11.1 & 476140 & 12.8 \\
\hline Lower Limit & & & 331856 & 4.96 & 180236 & 6.5 & 227611 & 7.79 & 183049 & 10.1 & 118035 & 11.8 \\
\hline Lab Sample ID & & & & & & & & & & & & \\
\hline EX120217-8MB & & & 761285 & 5.48 & 360553 & 7.00 & 502621 & 8.28 & 374888 & 10.61 & 215323 & 12.25 \\
\hline EX120917-8LCS & & & 844780 & 5.48 & 409484 & 7.00 & 580786 & 8.29 & 451967 & 10.61 & 287731 & 12.28 \\
\hline EX120217-8LCSD & & & 875228 & 5.46 & 415221 & 7.00 & 602796 & 8.28 & 467762 & 10.61 & 270761 & 12.28 \\
\hline $1209154-3$ & & & 868120 & 5.48 & 421043 & 7.01 & 573427 & 8.30 & 408426 & 10.62 & 258498 & 12.28 \\
\hline $1209154-2$ & & & 845851 & 5.48 & 452767 & 7.01 & 561273 & 8.30 & 352897 & 10.61 & 215043 & 12.28 \\
\hline 1209154-2MS & & & 861130 & 5.47 & 505164 & 7.01 & 662884 & 8.30 & 502318 & 10.61 & 320076 & $\overline{12.28}$ \\
\hline 1209154-2MSD & & & 976219 & 5.48 & 504037 & 7.01 & 698484 & 8.30 & 585857 & 10.61 & 417970 & 12.28 \\
\hline $1209154-7$ & & & 930252 & 5.46 & 436304 & 7.00 & 624193 & 8.28 & 504627 & 10.61 & 367635 & 12.26 \\
\hline $1209154-11$ & & & 1017470 & 5.48 & 471365 & 7.00 & 620072 & 8.30 & 638213 & 10.61 & 448258 & 12.28 \\
\hline $1209154-6$ & & & 841908 & 5.48 & 440781 & 7.00 & 577514 & 8.29 & 597092 & 10.61 & 363068 & 12.28 \\
\hline $1209154-10$ & & & 829303 & 5.48 & 434087 & 7.00 & 550419 & 8.29 & 491494 & 10.61 & 323058 & 12.26 \\
\hline $1209154-6 \mathrm{RR} 1$ & & & 859889 & 5.48 & 455237 & 7.00 & 591849 & 8.30 & 688989 & \begin{tabular}{|l|}
10.61 \\
\end{tabular} & 452921 & 12.28 \\
\hline
\end{tabular}




\section{A}

\section{Supporting Raw Data}




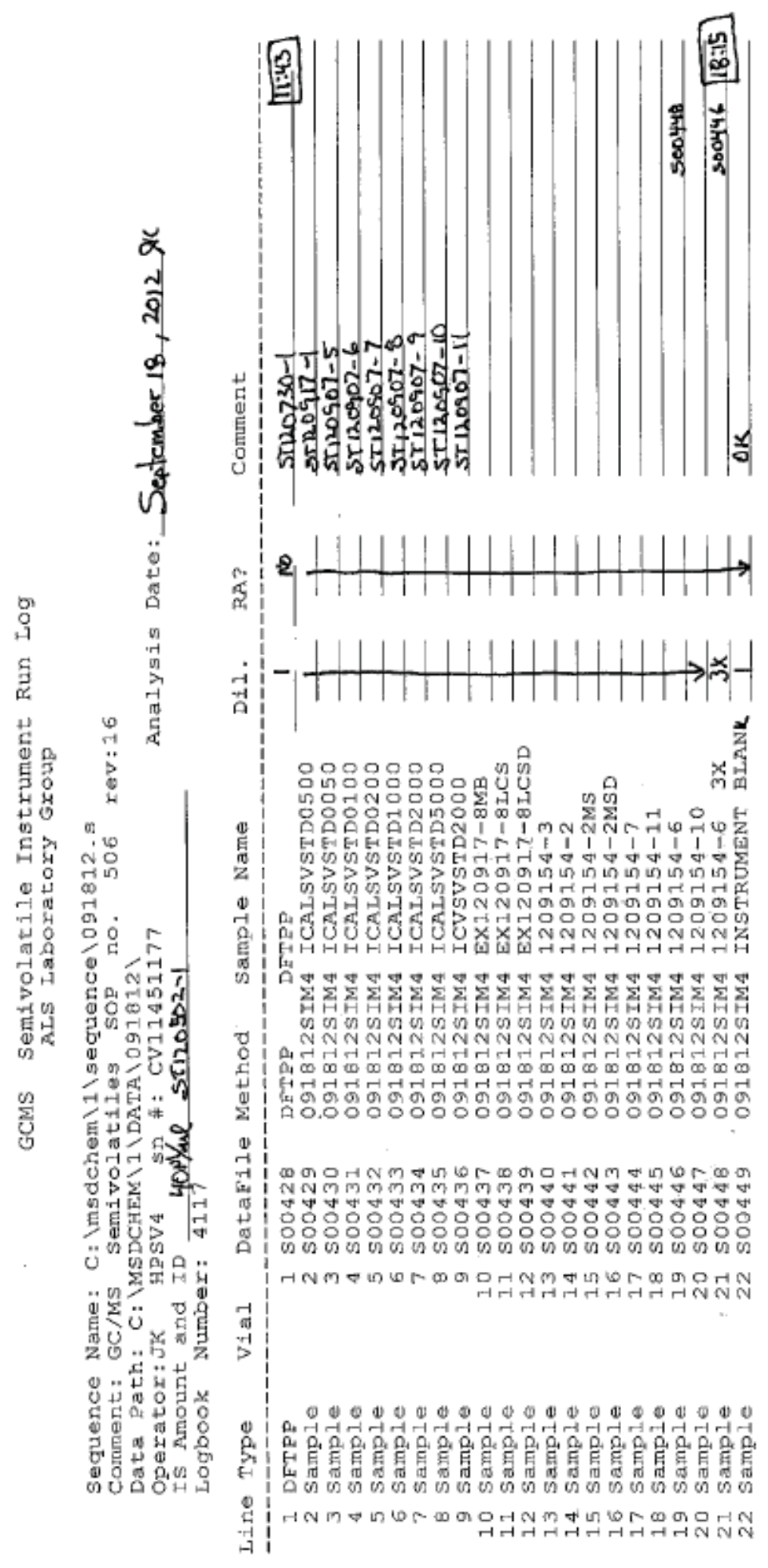

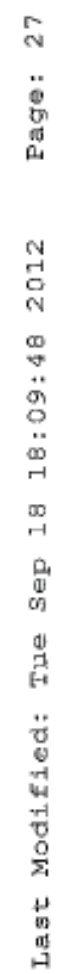

33 of 33 


\title{
GC/MS Volatiles
}

\author{
Case Narrative
}

\section{Battelle Energy Alliance \\ MFC Biodiesel Tank Removal , TOS-A1175 -- BEA030488}

Work Order Number: 1209154

1. This report consists of 6 soil samples. The samples were received cool and intact by ALS on $09 / 12 / 12$.

2. These samples were prepared according to SW-846, 3rd Edition procedures. Specifically, the soil samples were prepared using a heated purge based on Method 5035A.

3. The samples were analyzed using GC/MS with an RTX-624, RTX-VMS, or equivalent capillary column according to the current revision of SOP 525 based on SW-846 Method 8260 . All positive results were quantitated against the initial calibration standards using the internal standard technique. The identification of positive results was achieved by a comparison of the retention time and mass spectrum of the sample versus the daily calibration standard.

4. All initial calibration criteria were met.

5. All initial calibrations are verified by comparing a second source standard calibration verification (ICV) against the calibration curve. All criteria for initial calibration verification were met.

6. All criteria were met in daily (continuing) calibration verifications (CCV).

7. Methylene chloride, acetone and 2-butanone are common laboratory contaminants. In order to minimize the levels of these compounds detected in the $\mathrm{gc} / \mathrm{ms}$ analysis, ALS has designated its volatile laboratory as a restricted access area. In addition, the laboratory has been equipped with a dedicated, air intake and exhaust system that operates under positive pressure in order to minimize cross contamination of these compounds. Due to fluctuations in ambient laboratory conditions, reported sample values for common laboratory contaminants may be due to lab contamination even if the compound in question is not detected in the associated method blank.

All method blank criteria were met. 
8. All laboratory control sample and laboratory control sample duplicate recoveries and RPDs were within the acceptance criteria.

9. Sample 1209154-1 was designated as the quality control sample for this analysis. Similarity of matrix and therefore relevance of the $\mathrm{QC}$ results should not be automatically inferred for any sample other than the native sample selected for QC.

All matrix spike and matrlx spike duplicate recoveries and RPDs were within acceptance criteria with the following exceptions:

\begin{tabular}{|c|c|c|}
\hline Spiked Compound & QC Sample & Direction \\
\hline Ethylbenzene & MS & Low \\
\hline M+P.Xylene & MS & Low \\
\hline O-Xylene & MS & Low \\
\hline
\end{tabular}

The recoveries of these compounds in the laboratory control sample and laboratory control sample duplicate were within control limits, which suggest the outliers in the matrix spikes may have been due to matrix effects. No further action was taken.

10. The samples were analyzed within the established holding time.

11. All surrogate recoveries were within acceptance criteria.

12. All internal standard recoveries were within acceptance criteria.

13. Manual integrations are performed when needed to provide consistent and defensible data following the guidelines in the current revision of SOP 939.

The data contained in the following report have been reviewed and approved by the personnel listed below. In addition, ALS certifies that the analyses reported herein are true, complete and correct within the limits of the methods employed.

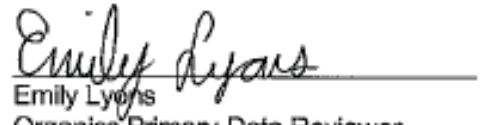

Organics Primary Data Reviewer

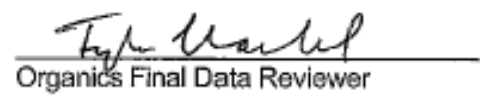

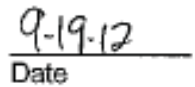

$9-19-12$ 


\title{
$A$
}

\author{
ALS \\ Data Qualifier Flags \\ Chromatography and Mass Spectrometry
}

U or ND: This flag indicates that the compound was analyzed for but not detected.

$\mathrm{J}: \quad$ This flag indicates an estimated value. This flag is used as follows : (1) when estimating a concentration for tentatively identified compounds (TICs) where a 1:1 response is assumed; (2) when the mass spectral and retention time data indicate the presence of a compound that meets the volatile and semivolatile GC/MS identification criteria, and the result is less than the reporting limit (RL) but greater than the method detection limit (MDL); (3) when the retention time data indicate the presence of a compound that meets the GC identification criteria, and the result is less than the RL but greater than the MDL; and (4) the reported value is estimated.

B: $\quad$ This flag is used when the analyte is detected in the associated method blank as well as in the sample. It indicates probable blank contamination and warns the data user. This flag shall be used for a tentatively identified compound (TIC) as well as for a positively identified target compound.

E: $\quad$ This flag identifies compounds whose concentration exceeds the upper level of the calibration range.

A: This flag indicates that a tentatively identified compound is a suspected aldol-condensation product.

$\mathrm{X}$ : This flag indicates that the analyte was diluted below an accurate quantitation level.

*: This flag indicates that a spike recovery is equal to or outside the control criteria used.

+: $\quad$ This flag indicates that the relative percent difference (RPD) equals or exceeds the control criteria. 


\section{A}

\section{Chain of Custody}




\section{ALS Environmental -- FC}

\section{Sample Number(s) Cross-Reference Table}

\begin{tabular}{|c|c|c|c|c|c|}
\hline \multicolumn{6}{|c|}{$\begin{array}{c}\text { OrderNum: } 1209154 \\
\text { Client Name: Battelle Energy Alliance } \\
\text { Client Project Name: MFC Biodiesel Tank Removal ,TOS-A1175 } \\
\text { Client Project Number: BEA030488 } \\
\text { Client PO Number: SOW-8500 }\end{array}$} \\
\hline $\begin{array}{c}\text { Client Sample } \\
\text { Number }\end{array}$ & $\begin{array}{c}\text { Lab Sample } \\
\text { Number }\end{array}$ & COC Number & Matrix & \begin{tabular}{|c|} 
Date \\
Collected
\end{tabular} & $\begin{array}{c}\text { Time } \\
\text { Collected }\end{array}$ \\
\hline BEA030488_MFC & $1209154-1$ & & SOIL & 10-Sep-12 & $14: 36$ \\
\hline BEA030489_MFC & $1209154-2$ & & SOIL & 10-Sep-12 & $14: 36$ \\
\hline BEA030490_MFC & $1209154-3$ & & SOIL & 10-Sep-12 & $14: 22$ \\
\hline BEA030491_MFC & $1209154-4$ & & SOIL & 10-Sep-12 & $14: 22$ \\
\hline BEA030492_MFC & $1209154-5$ & & SOIL & $10-$ Sep-12 & $13: 41$ \\
\hline BEA030493_MFC & $1209154-6$ & & SOIL & 10-Sep-12 & $13: 41$ \\
\hline BEA030494_MFC & $1209154-7$ & & SOIL & 10-Sep-12 & 13:55 \\
\hline BEA030495_MFC & $1209154-8$ & & SOIL & 10-Sep-12 & 13:55 \\
\hline BEA030496_MFC & $1209154-9$ & & SOIL & $10-$ Sep-12 & $14: 44$ \\
\hline BEA030497_MFC & $1209154-10$ & & SOIL & 10-Sep-12 & $14: 44$ \\
\hline BEA030498_MFC & $1209154-11$ & & SOIL & 10-Sep-12 & $14: 28$ \\
\hline BEA030499_MFC & $1209154-12$ & & SOIL & 10-Sep-12 & $14: 28$ \\
\hline
\end{tabular}




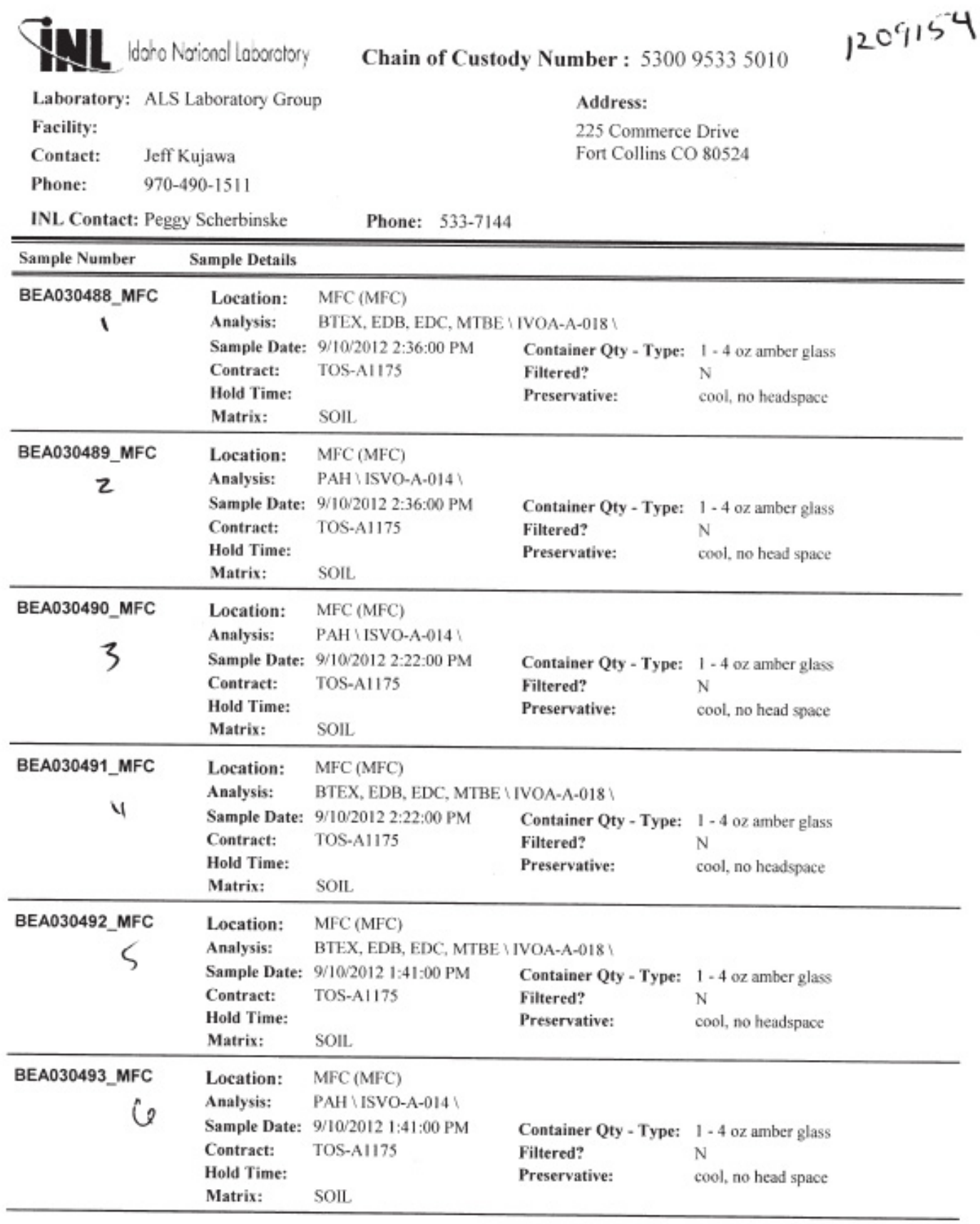




\begin{tabular}{|c|c|c|c|c|}
\hline \multirow[b]{2}{*}{$\begin{array}{c}\text { BEA030494_MFC } \\
-1\end{array}$} & nol Loborctory & \multicolumn{3}{|c|}{ Chain of Custody Number : 530095335010} \\
\hline & $\begin{array}{l}\text { Location: } \\
\text { Analysis: } \\
\text { Sample Date: } \\
\text { Contract: } \\
\text { Hold Time: } \\
\text { Matrix: }\end{array}$ & $\begin{array}{l}\text { MFC (MFC) } \\
\text { PAH \ISVO-A-014 } \\
9 / 10 / 20121: 55: 00 \mathrm{PM} \\
\text { TOS-A1175 } \\
\text { SOIL }\end{array}$ & $\begin{array}{l}\text { Container Qty - Type: } \\
\text { Filtered? } \\
\text { Preservative: }\end{array}$ & $\begin{array}{l}\text { I - } 4 \text { oz amber glass } \\
\mathrm{N} \\
\text { cool, no head space }\end{array}$ \\
\hline BEA030495_MFC & $\begin{array}{l}\text { Location: } \\
\text { Analysis: } \\
\text { Sample Date: } \\
\text { Contract: } \\
\text { Hold Time: } \\
\text { Matrix: }\end{array}$ & $\begin{array}{l}\text { MFC (MFC) } \\
\text { BTEX, EDB, EDC, MT1 } \\
\text { 9/10/2012 1:55:00 PM } \\
\text { TOS-A1175 } \\
\text { SOIL }\end{array}$ & $\begin{array}{l}\text { [VOA-A-018 } \\
\text { Container Qty - Type: } \\
\text { Filtered? } \\
\text { Preservative: }\end{array}$ & $\begin{array}{l}\text { I- } 4 \text { oz amber glass } \\
\mathrm{N} \\
\text { cool, no headspace }\end{array}$ \\
\hline $\begin{array}{c}\text { BEA030496_MFC } \\
9\end{array}$ & $\begin{array}{l}\text { Location: } \\
\text { Analysis: } \\
\text { Sample Date: } \\
\text { Contract: } \\
\text { Hold Time: } \\
\text { Matrix: }\end{array}$ & $\begin{array}{l}\text { MFC (MFC) } \\
\text { BTEX, EDB, EDC. MTI } \\
\text { 9/10:2012 2:44:00 PM } \\
\text { TOS-A1175 } \\
\text { SOIL. }\end{array}$ & $\begin{array}{l}\text { IVOA-A-018 } \\
\text { Container Qty - Type: } \\
\text { Filtered? } \\
\text { Preservative: }\end{array}$ & $\begin{array}{l}\text { 1-4 oz amber glass } \\
\mathrm{N} \\
\text { cool, no headspace }\end{array}$ \\
\hline $\begin{array}{c}\text { BEA030497_MFC } \\
, 0\end{array}$ & $\begin{array}{l}\text { Location: } \\
\text { Analysis: } \\
\text { Sample Date: } \\
\text { Contract: } \\
\text { Hold Time: } \\
\text { Matrix: }\end{array}$ & $\begin{array}{l}\text { MFC (MFC) } \\
\text { PAH } \backslash 1 S V O-A=014 \backslash \\
9 / 10 / 20122: 44: 00 \mathrm{PM} \\
\text { TOS-A1175 } \\
\text { SOIL. }\end{array}$ & $\begin{array}{l}\text { Container Qty - Type: } \\
\text { Filtered? } \\
\text { Preservative: }\end{array}$ & $\begin{array}{l}1-4 \mathrm{oz} \text { amber glass } \\
\mathrm{N} \\
\text { cool, no head space }\end{array}$ \\
\hline BEA030498_MFC & $\begin{array}{l}\text { Location: } \\
\text { Analysis: } \\
\text { Sample Date: } \\
\text { Contract: } \\
\text { Hold Time: } \\
\text { Matrix: }\end{array}$ & $\begin{array}{l}\text { MFC (MFC) } \\
\text { PAH } \backslash \text { ISVO-A-014 } \\
\text { 9/10/2012 2:28:00 PM } \\
\text { TOS-A1175 } \\
\text { SOIL }\end{array}$ & $\begin{array}{l}\text { Container Qty - Type: } \\
\text { Filtered? } \\
\text { Preservative: }\end{array}$ & $\begin{array}{l}\text { 1-4 oz amber glass } \\
\mathrm{N} \\
\text { cool, no head space }\end{array}$ \\
\hline BEA030499_MFC & $\begin{array}{l}\text { Location: } \\
\text { Analysis: } \\
\text { Sample Date: } \\
\text { Contract: } \\
\text { Hold Time: } \\
\text { Matrix: }\end{array}$ & $\begin{array}{l}\text { MFC (MFC) } \\
\text { BTEX, EDB, EDC, MTt } \\
\text { 9/10/2012 2:28:00 PM } \\
\text { TOS-A1175 } \\
\text { SOIL }\end{array}$ & $\begin{array}{l}\text { IVOA-A-018 } \\
\text { Container Qty - Type: } \\
\text { Filtered? } \\
\text { Preservative: }\end{array}$ & $\begin{array}{l}1-4 \text { oz amber glass } \\
\mathrm{N} \\
\text { cool, no headspace }\end{array}$ \\
\hline
\end{tabular}


L doho Notionol Loborciory Chain of Custody Number : 530095335010

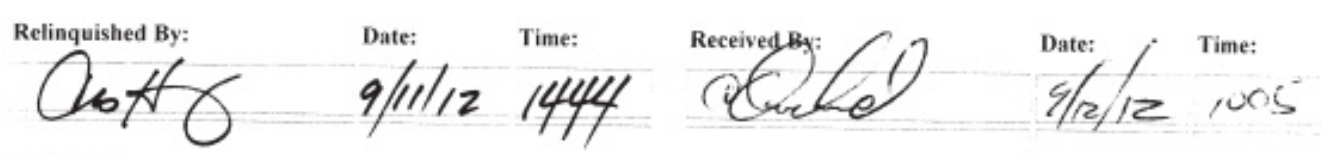

Comments:

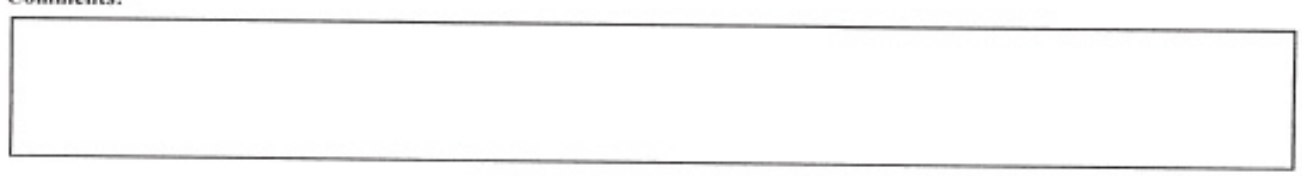

Monday, September 10, 2012

Pageaf'36

70 


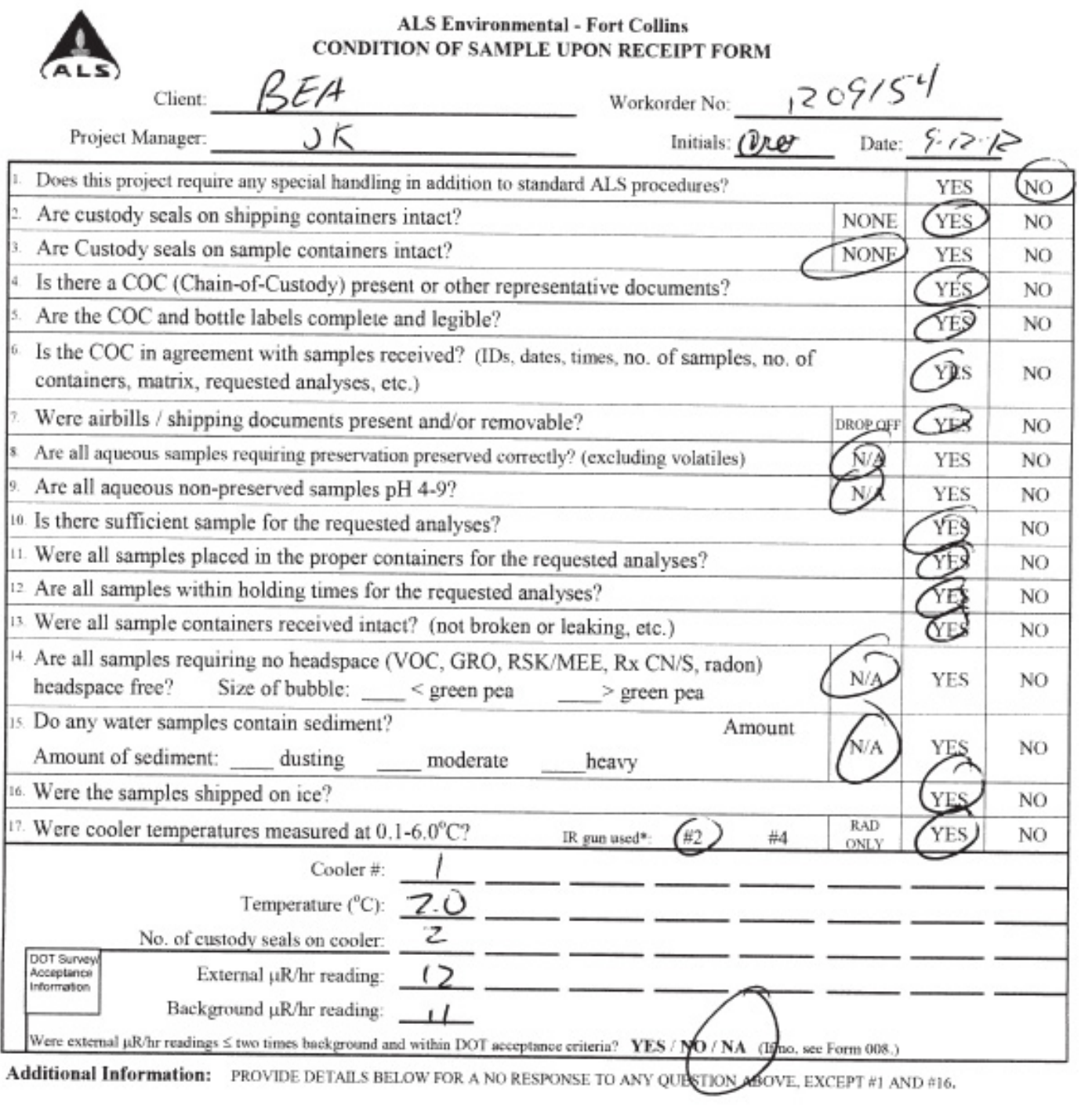

\footnotetext{
If applicnble. was the elient contacted? YES / NO (NA Contact:
Project Manager Signature / Date:
Form 20 Ir24.xls (0604/2012)
} 


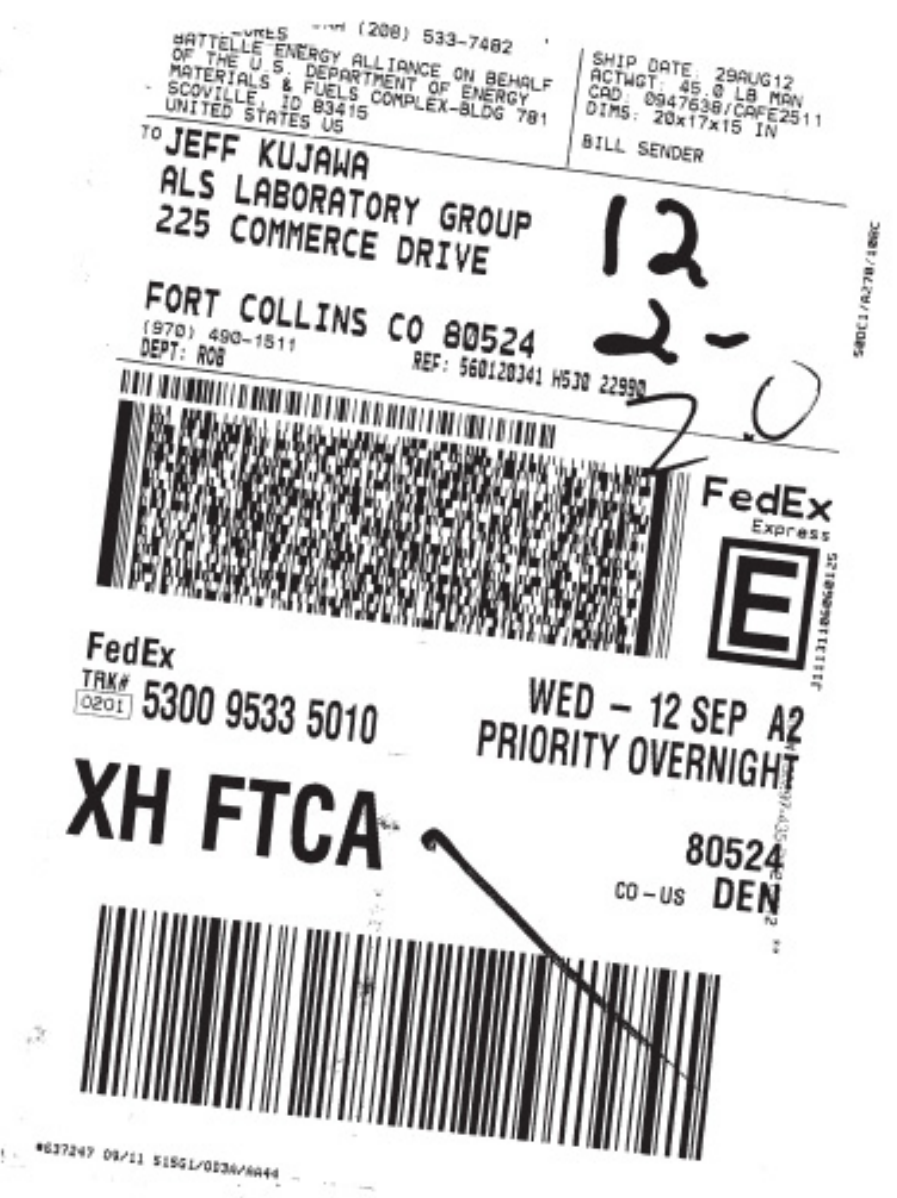

10 of 36 


\section{A}

\section{Analytical Results}




\section{GC/MS Volatiles}

Method SW8260C

Method Blank

Lab Name: ALS Environmental - FC

Work Order Number: 1209154

Client Name: Battele Energy Alliance

ClientProject ID: MFC Biodiesel Tank Removal ,TOS-A1175 BEA030488

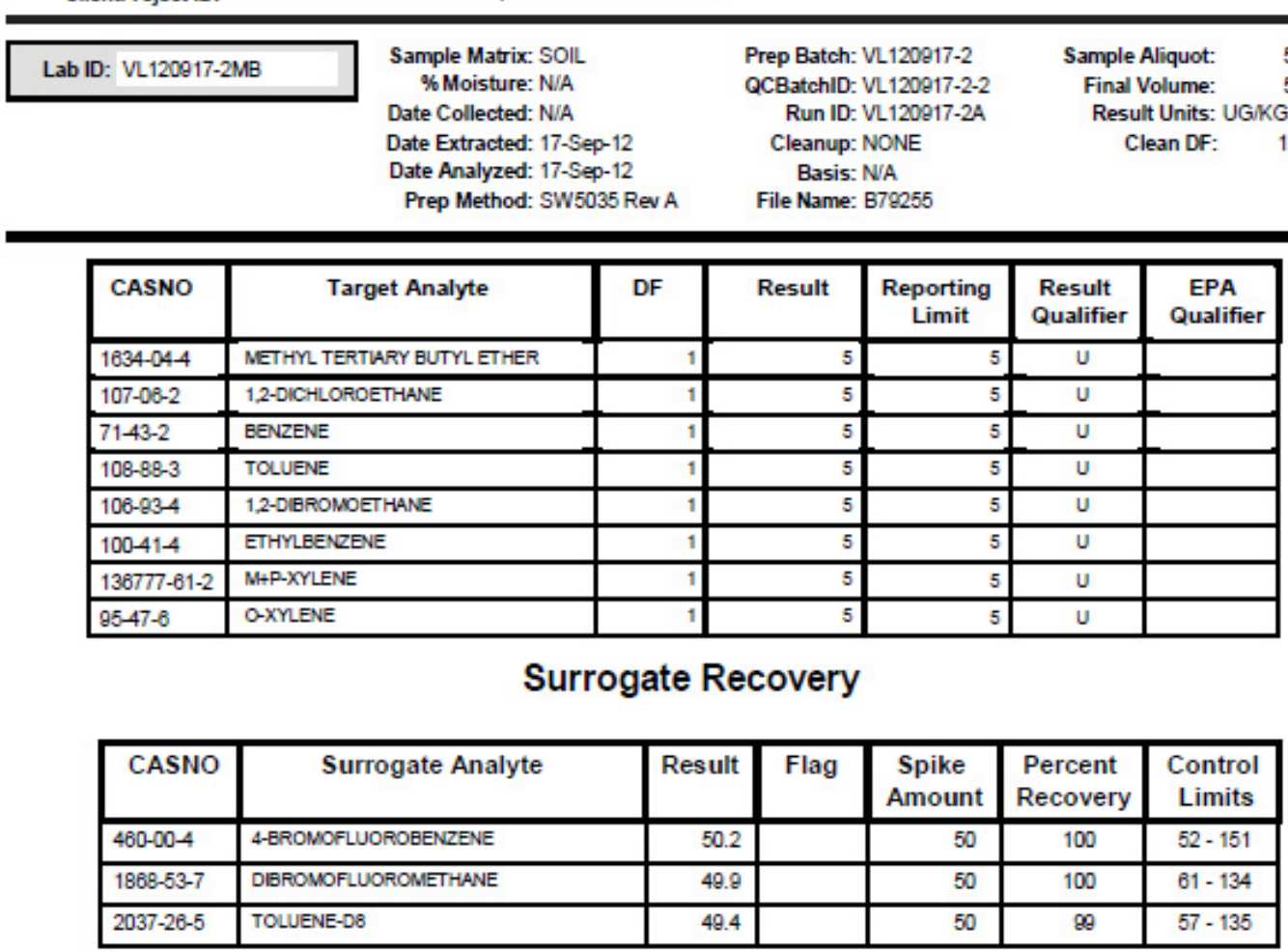

Data Package ID: VL1209154-1

Date Printed: Wecinesday, September 19, 2012

ALS Environmental -- FC

Page 1 of 1

LIMS Version: 6.611

12 of 36 


\title{
GC/MS Volatiles
}

\author{
Method SW8260 Revision C
}

Sample Results

Lab Name: ALS Environmental - FC

Work Order Number: 1209154

Client Name: Battele Energy Alliance

ClientProject ID: MFC Biodiesel Tank Remova ,TOS-A1175 BEA030488

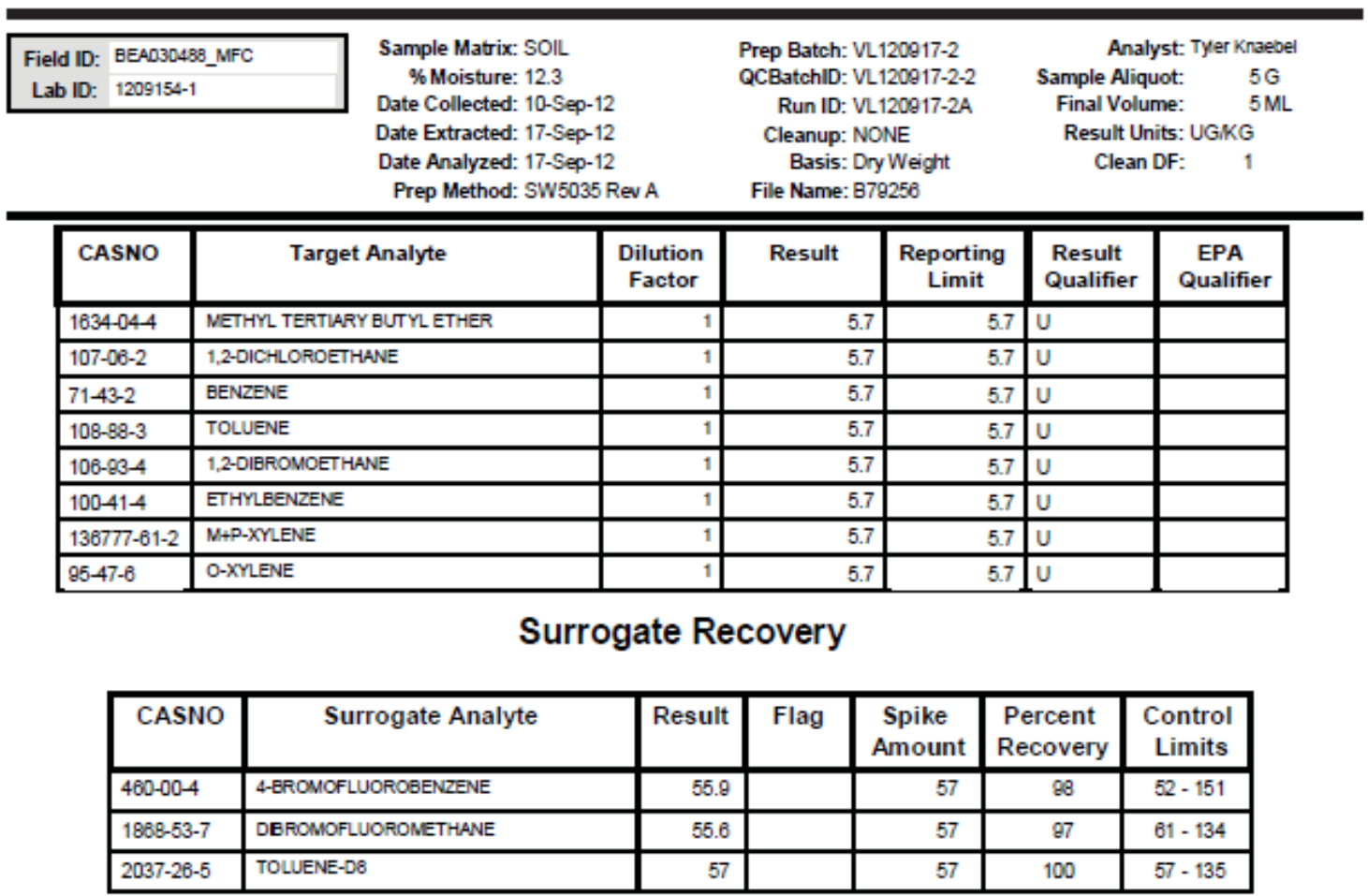

Data Package ID: VL1209154-1

$\begin{array}{lcc}\text { Date Printed: Wednesday, September 19, } 2012 & \text { ALS Environmental -- FC } \\ \text { LIMS Version: } 6.611 & \text { Page } 1 \text { of } 6\end{array}$

13 of 36 


\section{GC/MS Volatiles}

\section{Method SW8260 Revision C Sample Results}

Lab Name: ALS Environmental - FC

Work Order Number: 1209154

Client Name: Battele Energy Alliance

ClientProject ID: MFC Biodiesel Tank Removal ,TOS-A1175 BEA030488

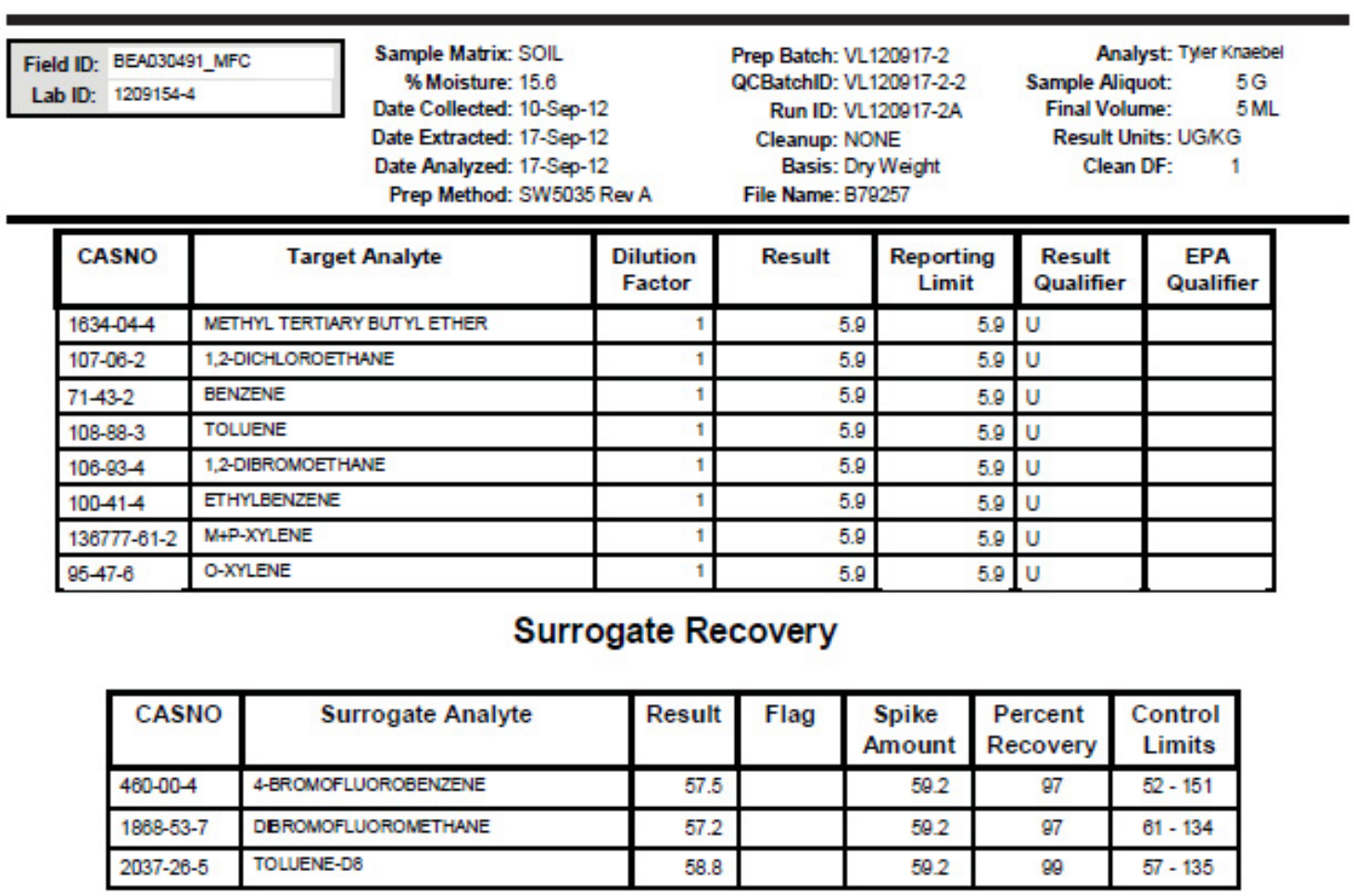

Data Package ID: VL1209154-1

\begin{tabular}{|c|c|}
\hline Date Printed: Wecinesday. September 19, 2012 & $\begin{array}{l}\text { ALS Environmental -- FC } \\
\text { LMS version: } 6.611\end{array}$ \\
\hline
\end{tabular}

14 of 36 


\section{GC/MS Volatiles}

\section{Method SW8260 Revision C Sample Results}

Lab Name: ALS Environmental - FC

Work Order Number: 1209154

Client Name: Battele Energy Alliance

ClientProject ID: MFC Biodiesel Tank Removal ,TOS-A1175 BEA030488

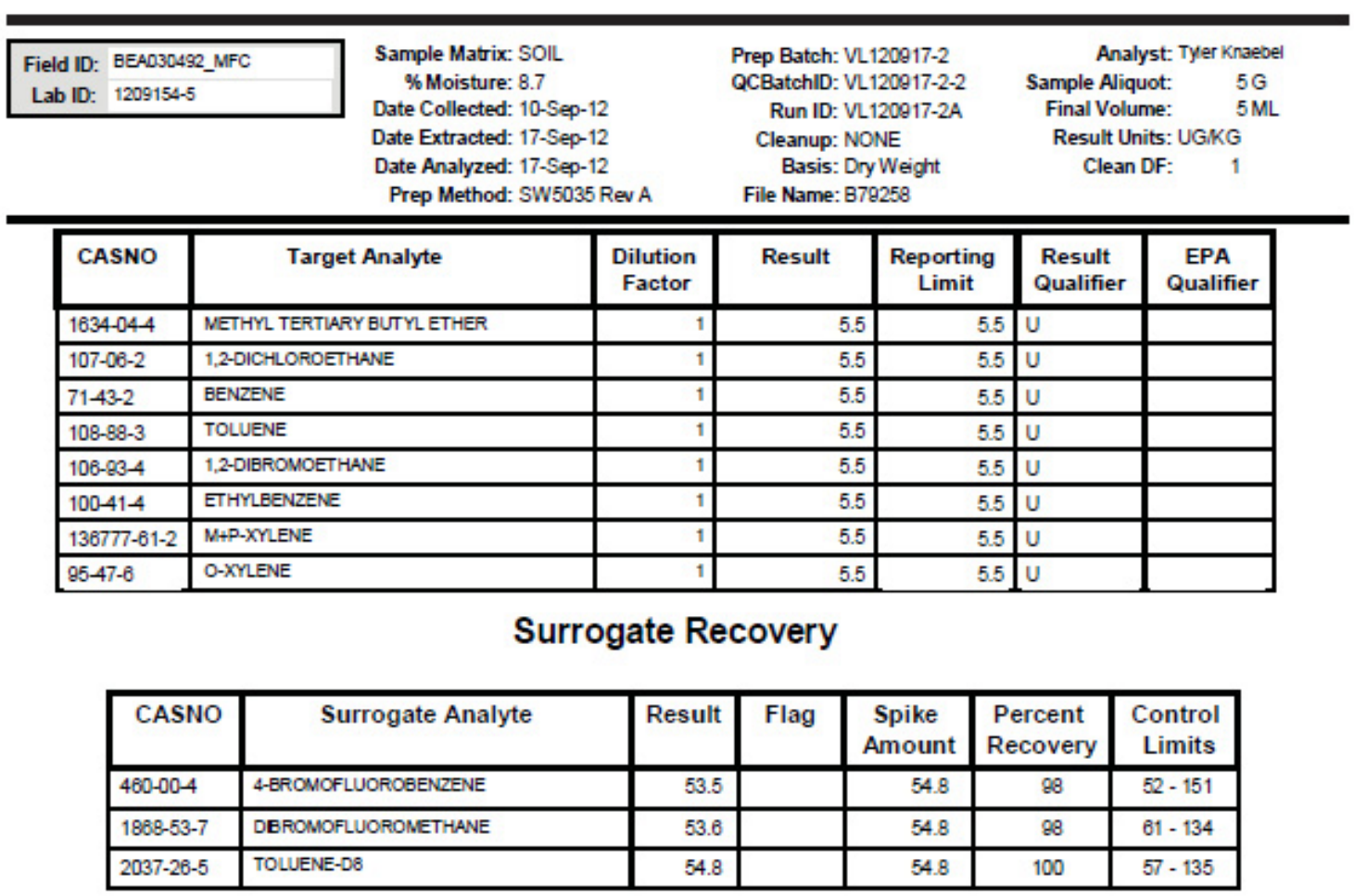

Data Package ID: VL1209154-1

$\begin{array}{ccc}\text { Date Printed: Wednesday, September 19, } 2012 & \text { ALS Environmental -- FC } \\ \text { LimS Version: } 6.611 & \text { Page } 3 \text { of } 6\end{array}$

15 of 36 


\section{GC/MS Volatiles}

\section{Method SW8260 Revision C Sample Results}

Lab Name: ALS Environmental - FC

Work Order Number: 1209154

Client Name: Battele Energy Alliance

ClientProject ID: MFC Biodiesel Tank Removal ,TOS-A1175 BEA030488

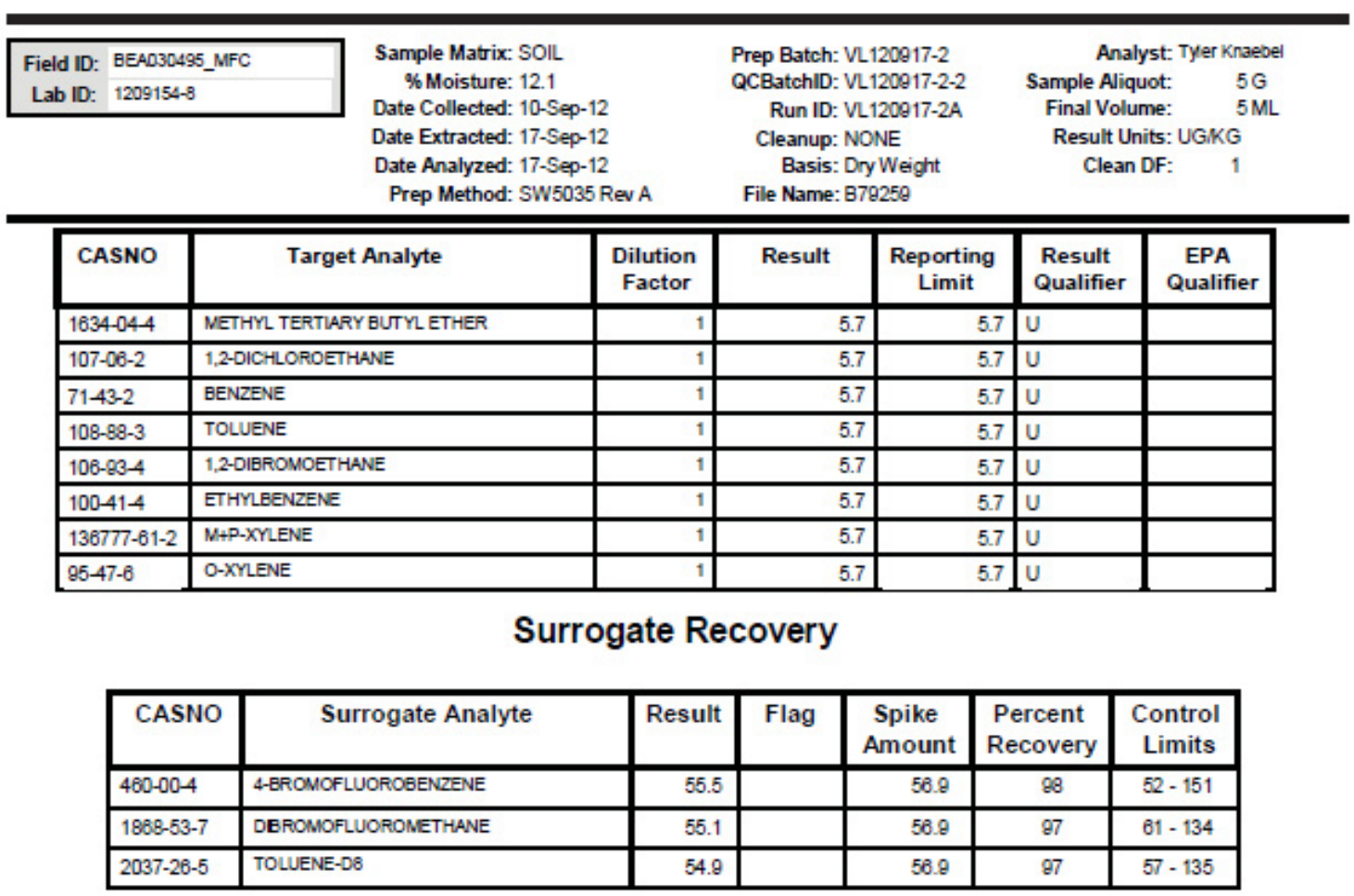

Data Package ID: VL1209154-1

$\begin{array}{lcc}\text { Date Printed: Wechesday, September 19, } 2012 & \text { ALS Environmental -- FC } \\ \text { LMS Version: } 6.611 & \text { Page } 4 \text { of } 6\end{array}$

16 of 36 


\section{जUiniv vuiailes}

Method SW8260 Revision C

\section{Sample Results}

Lab Name: ALS Environmental - FC

Work Order Number: 1209154

Client Name: Battele Energy Alliance

ClientProject ID: MFC Biodiesel Tank Remova ,TOS-A1175 BEA030488

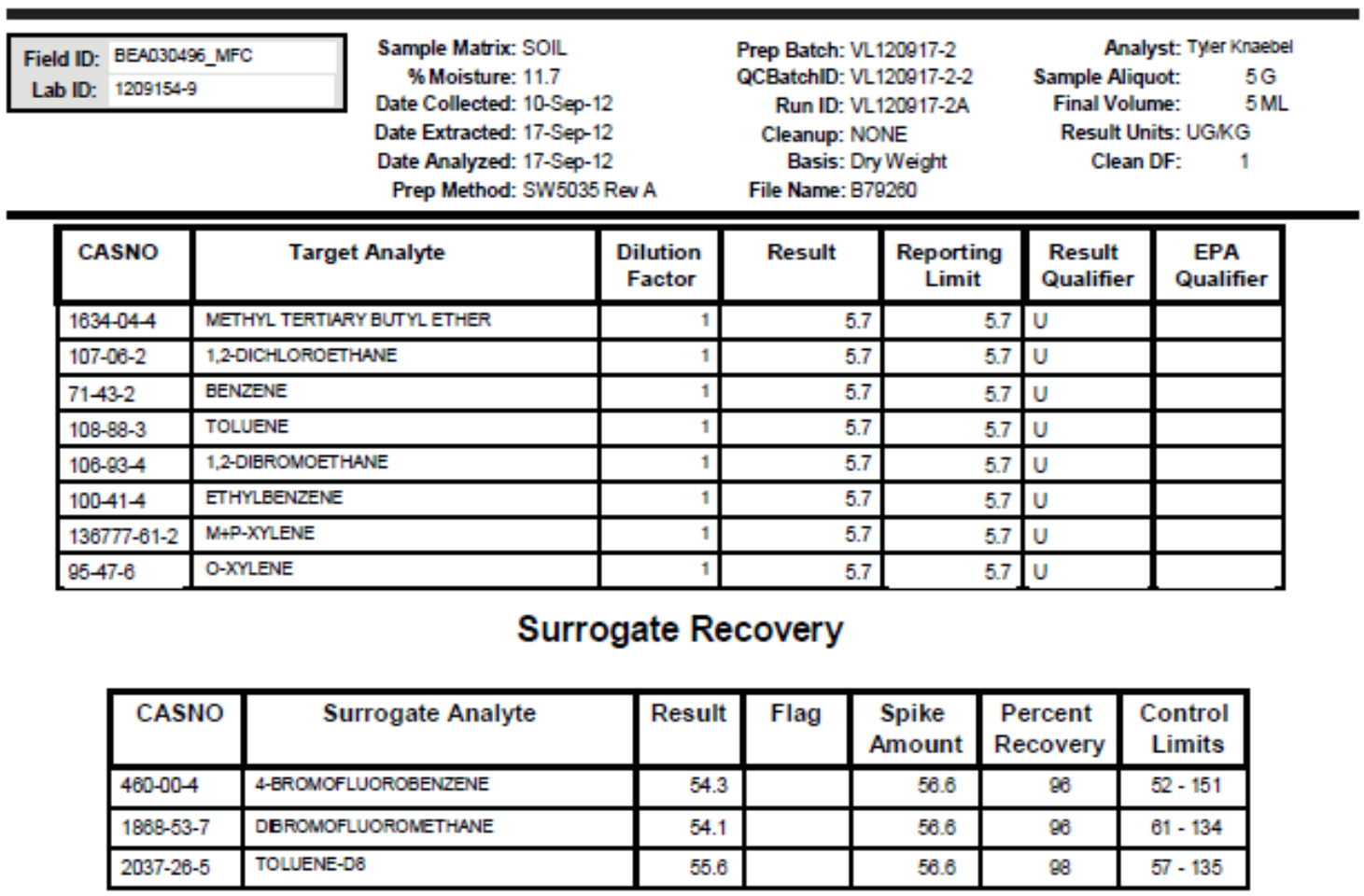

Data Package ID: VL1209154-1

$\begin{array}{lcc}\text { Date Printed: Wednesday, September 19, } 2012 & \text { ALS Environmental -- FC } \\ \text { LMS Version: } 6.611 & \text { Page } 5 \text { of } 6\end{array}$

17 of 36 


\section{GC/MS Volatiles}

Method SW8260 Revision C

Sample Results

Lab Name: ALS Environmental - FC

Work Order Number: 1209154

Client Name: Battele Energy Alliance

ClientProject ID: MFC Biodiesel Tank Remova ,TOS-A1175 BEA030488

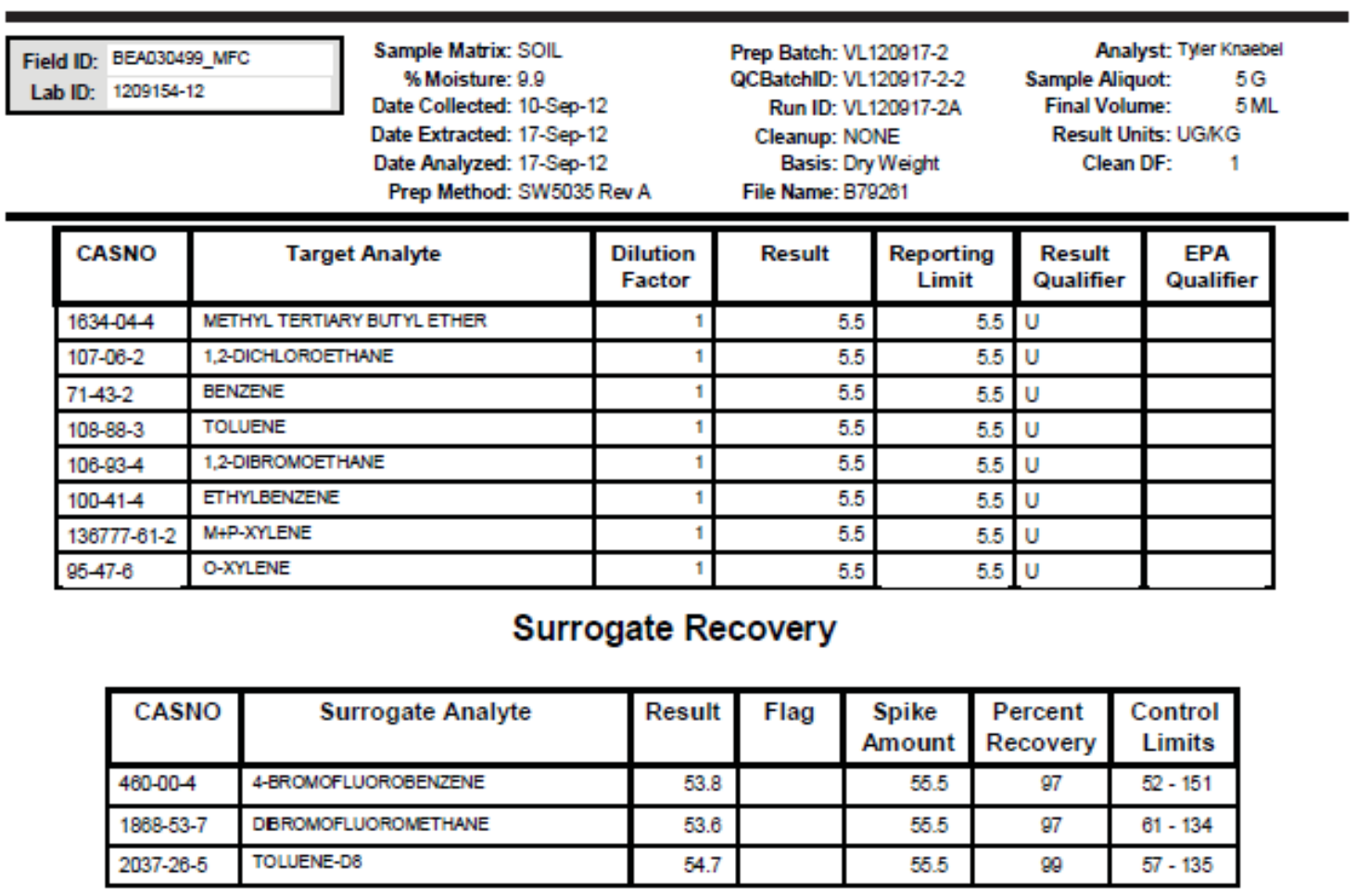

Data Package ID: VL1209154-1

$\begin{array}{lcc}\text { Date Printed: Wednesday, Septenber 19, } 2012 & \text { ALS Environmental -- FC } & \text { Page } 6 \text { of } 6\end{array}$

18 of 36 


\section{A A}

Supporting QA/QC Data

19 of 36 


\section{Surrogate Summary for GC/MS Volatiles}

Method SW8260C

Lab Name: ALS Environmental - FC

Work Order Number: 1209154

Client Name: Battelle Energy Alliance

ClientProject ID: MFC Biodiesel Tank Removal ,TOS-A1175 BEA030488

\begin{tabular}{|c|c|c|c|}
\hline \multirow{2}{*}{$\begin{array}{l}\text { PrepBatchID: VL120917-2 } \\
\text { QC Batch ID: VL120917-2-2 }\end{array}$} & Surrogate Compound & \multicolumn{2}{|c|}{ Control Limits } \\
\hline & Dibromofluoromethane & 61 & 134 \\
\hline Date Extracted: 9/17/2012 & Toluene-d8 & 57 & 135 \\
\hline & 4-Bromofluorobenzene & 52 & 151 \\
\hline & 1,2-dichloroethane-d4 & & \\
\hline
\end{tabular}

\begin{tabular}{|c|c|c|c|c|c|c|c|}
\hline Lab ID & Client Sample ID & $\begin{array}{c}\text { Date } \\
\text { Collected }\end{array}$ & $\begin{array}{c}\text { Date } \\
\text { Received }\end{array}$ & $\begin{array}{l}\text { DBFM \% } \\
\text { Recovery }\end{array}$ & $\begin{array}{l}\text { BZMEDB \% } \\
\text { Recovery }\end{array}$ & $\begin{array}{l}\text { BRAFBZ \% } \\
\text { R日covery }\end{array}$ & $\begin{array}{l}\text { 12DCED4 \% } \\
\text { Recovery }\end{array}$ \\
\hline VL120917-2LCS & $x 0000000$ & NA & $2000000 x$ & 102 & 97 & 100 & \\
\hline VL120917-2LCSD & $x 000000$ & NA & $x \times 10000 X$ & 100 & 98 & 101 & \\
\hline VL120917-2NB & $x 000000 x$ & NA & $1000000 x$ & 100 & 99 & 100 & \\
\hline $1209154-9$ & BEA030496_MFC & 9/10/2012 & $9 / 12 / 2012$ & 96 & 98 & 98 & \\
\hline $1209154-8$ & BEAD30496_MFC & $9 / 10 / 2012$ & 9/12/2012 & 97 & 97 & 88 & \\
\hline $1209154-5$ & BEA030492_NFC & 9/10/2012 & $9 / 12 / 2012$ & 98 & 100 & 98 & \\
\hline $1209154-4$ & BEA030491_MFC & $9 / 10 / 2012$ & $9 / 12 / 2012$ & 97 & 99 & 97 & \\
\hline $1209154-12$ & BEA030499_MFC & 9/10/2012 & $9 / 12 / 2012$ & 97 & 99 & 97 & \\
\hline 1209154-1MS & BEAD30488_MFC & $9 / 10 / 2012$ & 9/12/2012 & 97 & 99 & 89 & \\
\hline 1209154-1MSD & BEA030488_NFC & 9/10/2012 & 9/12/2012 & 99 & 99 & 98 & \\
\hline $1209154-1$ & BEA030488_MFC & $9 / 10 / 2012$ & 9/12/2012 & 97 & 100 & 88 & \\
\hline
\end{tabular}

\begin{tabular}{|c|c|c|}
\hline Date Printed: Wednesday, September 19, 2012 & ALS Environmental -- FC & Page 1 of 1 \\
\hline Shaded values exceed established control limits. & LMAS Veraion: 6.611 & 20 of 36 \\
\hline
\end{tabular}




\section{GC/MS Volatiles}

Method SW8260C

Laboratory Control Sample and Laboratory Control Sample Duplicate

Lab Name: ALS Environmental - FC

Work Order Number: 1209154

Client Name: Battele Energy Alliance

ClientProject ID: MFC Biodiesel Tank Removal ,TOS-A1175 BEA030488

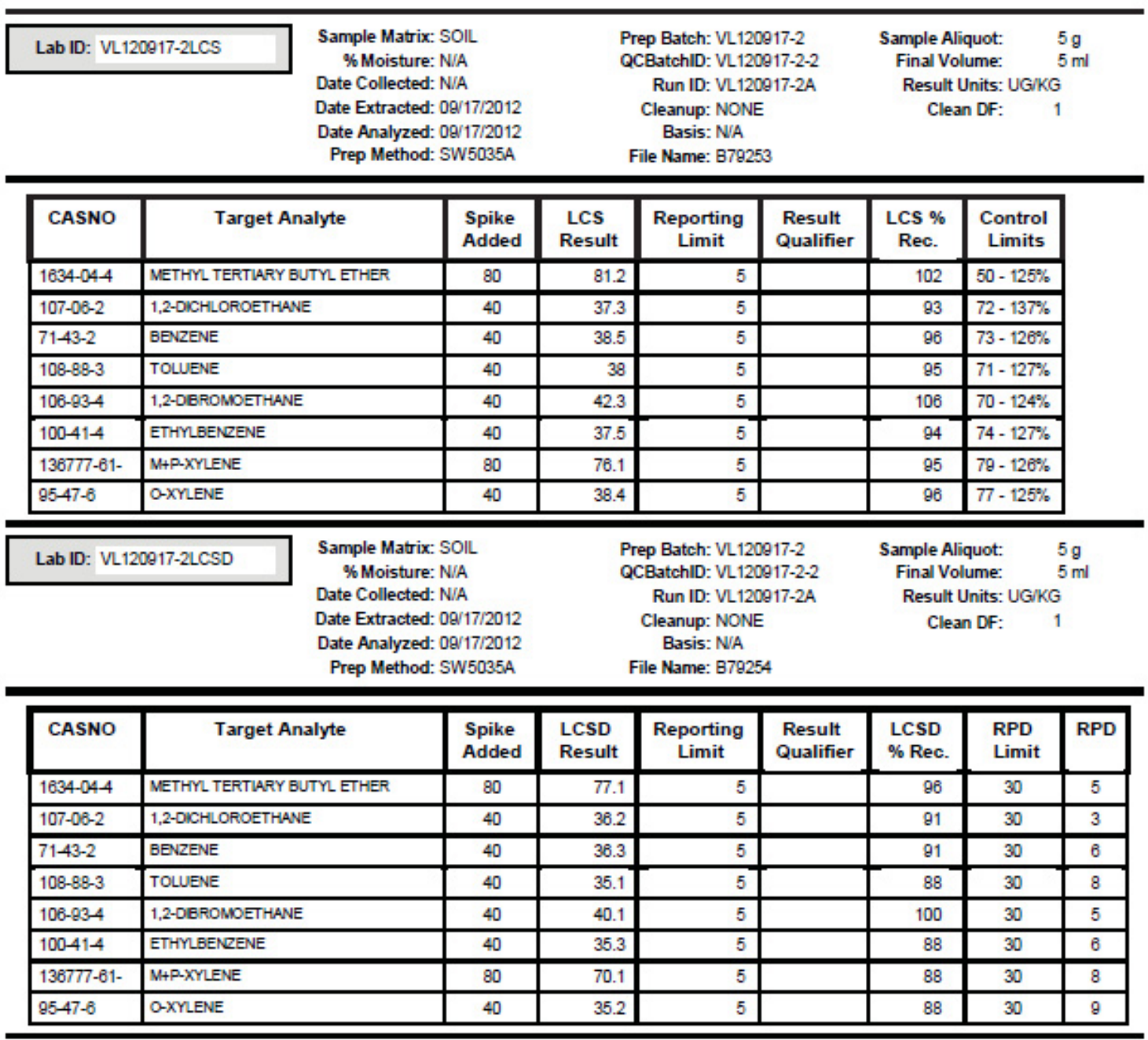

Data Package ID: VL1209154-1

\begin{tabular}{|c|c|}
\hline Date Printed: Wednesday, September 19, 2012 & $\begin{array}{c}\text { ALS Environmental - FC } \\
\text { uMs version: } 6.611\end{array}$ \\
\hline
\end{tabular}

21 of 36 


\section{GC/MS Volatiles}

Method SW8260C

Laboratory Control Sample and Laboratory Control Sample Duplicate

Lab Name: ALS Environmental - FC

Work Order Number: 1209154

Client Name: Battele Energy Alliance

ClientProject ID: MFC Biodiesel Tank Removal ,TOS-A1175 BEA030488

Surrogate Recovery LCS/LCSD

\begin{tabular}{|l|l|r|r|r|r|r|c|}
\hline CASNO & \multicolumn{1}{|c|}{ Target Analyte } & $\begin{array}{c}\text { Spike } \\
\text { Added }\end{array}$ & $\begin{array}{c}\text { LCS \% } \\
\text { Rec. }\end{array}$ & $\begin{array}{c}\text { LCS } \\
\text { Flag }\end{array}$ & $\begin{array}{c}\text { LCSD } \\
\% \text { Rec. }\end{array}$ & $\begin{array}{c}\text { LCSD } \\
\text { Flag }\end{array}$ & $\begin{array}{c}\text { Control } \\
\text { Limits }\end{array}$ \\
\hline $460-00-4$ & 4-EROMOFLUOROEENZENE & 50 & 100 & & 101 & & $52-151$ \\
\hline $1888-53-7$ & DigROMOFLUOROMETHANE & 50 & 102 & & 100 & & $61-134$ \\
\hline $2037-26-5$ & TOLUENE-D8 & 50 & 97 & & 98 & & $57-135$ \\
\hline
\end{tabular}

Data Package ID: VL1209154-1

Date Printed: Wednesday, September 19, $2012 \quad$ ALS Environmental - FC

220636 


\title{
GC/MS Volatiles
}

\author{
Method SW8260C
}

Matrix Spike And Matrix Spike Duplicate

Lab Name: ALS Environmental - FC

Work Order Number: 1209154

Client Name: Battelle Energy Alliance

ClientProject ID: MFC Biodiesel Tank Removal ,TOS-A1175 BEA030488

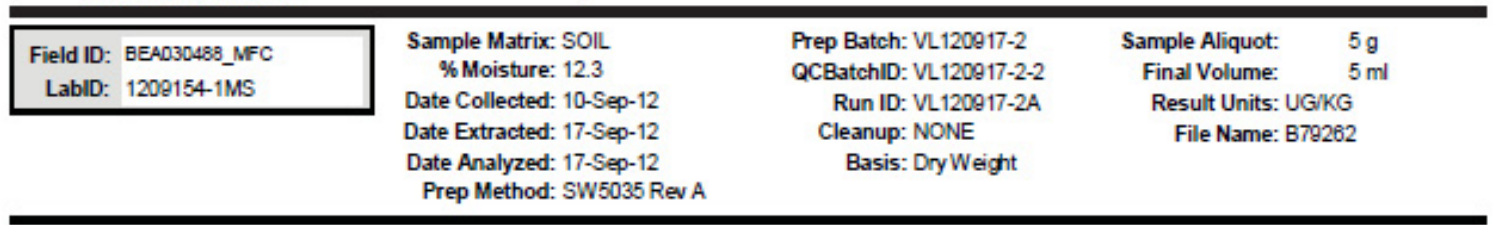

\begin{tabular}{|c|c|c|c|c|c|c|c|c|c|c|}
\hline CASNO & \multicolumn{2}{|c|}{ Target Analyte } & $\begin{array}{r}\text { Sample } \\
\text { Result }\end{array}$ & $\begin{array}{l}\text { Samp } \\
\text { Qual }\end{array}$ & $\begin{array}{c}\text { MS } \\
\text { Result }\end{array}$ & $\begin{array}{l}\text { MS } \\
\text { Qual }\end{array}$ & $\begin{array}{l}\text { Reporting } \\
\text { Limit }\end{array}$ & $\begin{array}{l}\text { Spike } \\
\text { Added }\end{array}$ & $\begin{array}{l}\text { MS \% } \\
\text { Rec. }\end{array}$ & $\begin{array}{l}\text { Control } \\
\text { Limits }\end{array}$ \\
\hline $1634-04-4$ & \multicolumn{2}{|c|}{ METHYL TERTLARY BUTYL ETHER } & 5.7 & u & 75.7 & & 5.7 & 91.2 & 83 & $50-125 \%$ \\
\hline $107-06-2$ & \multicolumn{2}{|l|}{ 1,2-DICHLOROETHANE } & 5.7 & u & 36.3 & & 5.7 & 45.6 & 80 & $72-137 \%$ \\
\hline $71-43-2$ & \multicolumn{2}{|l|}{ BENZENE } & 5.7 & u & 38.3 & & 5.7 & 45.6 & 84 & $73-126 \%$ \\
\hline $108-88-3$ & \multicolumn{2}{|l|}{ TOLUENE } & 5.7 & U & 37 & & 5.7 & 45.6 & 81 & $71-127 \%$ \\
\hline $106-93-4$ & \multicolumn{2}{|l|}{ 1,2-DEROMOETHANE } & 5.7 & u & 38.9 & & 5.7 & 45.6 & 85 & $70-124 \%$ \\
\hline $100-414$ & \multicolumn{2}{|l|}{ ETHYLBENZENE } & 5.7 & u & 33.4 & . & 5.7 & 45.6 & 73 & $74-127 \%$ \\
\hline $136777-61-$ & \multicolumn{2}{|l|}{ M+P-XYLENE } & 5.7 & u & 66.1 & . & 5.7 & 91.2 & 72 & $79-126 \%$ \\
\hline $85-47-6$ & \multicolumn{2}{|l|}{ OXYLENE } & 5.7 & U & 33.6 & 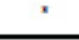 & 5.7 & 45.6 & 74 & $77-125 \%$ \\
\hline \multirow{3}{*}{\multicolumn{2}{|c|}{$\begin{array}{l}\text { Field ID: } \text { EEA030488_MFC } \\
\text { LabID: } 1209154-1 \text { MSD }\end{array}$}} & \multirow{3}{*}{\multicolumn{3}{|c|}{$\begin{array}{l}\text { Sample Matrix: SOIL } \\
\text { \% Moisture: } 12.3 \\
\text { Date Collected: } 10-\text { Sep-12 } \\
\text { Date Extracted: } 17-\text { Sep-12 } \\
\text { Date Analyzed: } 17-\text { Sep-12 } \\
\text { Prep Method: SW5035 Rev A }\end{array}$}} & \multicolumn{3}{|c|}{$\begin{array}{l}\text { Prep Batch: VL120917-2 } \\
\text { QCBatchID: VL120017-2-2 }\end{array}$} & \multicolumn{2}{|c|}{$\begin{array}{l}\text { Sample Aliquot: } \\
\text { Final Volume: }\end{array}$} & $\begin{array}{l}5 \mathrm{~g} \\
5 \mathrm{ml}\end{array}$ \\
\hline & & & & & Clear & up: NOI & & & ne: $B 7926$ & \\
\hline & & & & & & & & & & \\
\hline
\end{tabular}

\begin{tabular}{|l|l|r|r|r|r|r|c|c|}
\hline CASNO & \multicolumn{1}{|c|}{ Target Analyte } & $\begin{array}{c}\text { MSD } \\
\text { Result }\end{array}$ & $\begin{array}{c}\text { MSD } \\
\text { Qual }\end{array}$ & $\begin{array}{c}\text { Spike } \\
\text { Added }\end{array}$ & $\begin{array}{c}\text { MSD \% } \\
\text { Rec. }\end{array}$ & $\begin{array}{c}\text { Reporting } \\
\text { Limit }\end{array}$ & $\begin{array}{l}\text { RPD } \\
\text { Limit }\end{array}$ & RPD \\
\hline $1634-04-4$ & METHYL TERTIARY BUTYL ETHER & 89.2 & & 91.2 & 98 & 5.7 & 30 & 16 \\
\hline $107-06-2$ & 1,2-DICHLOROETHANE & 41.7 & & 45.6 & 91 & 5.7 & 30 & 14 \\
\hline $71-43-2$ & BENZENE & 43.2 & & 45.6 & 95 & 5.7 & 30 & 12 \\
\hline $108-88-3$ & TOLUENE & 43.4 & & 45.6 & 95 & 5.7 & 30 & 16 \\
\hline $106-93-4$ & 1.2-DEROMOETHANE & 45.6 & & 45.6 & 100 & 5.7 & 30 & 16 \\
\hline $100-41-4$ & ETHYLBENZENE & 40.2 & & 45.6 & 88 & 5.7 & 30 & 19 \\
\hline $136777-61-$ & M+P-XYLENE & 80.3 & & 91.2 & 88 & 5.7 & 30 & 19 \\
\hline $85-47-6$ & O-XYLENE & 40.9 & & 45.6 & 90 & 5.7 & 30 & 19 \\
\hline
\end{tabular}

Data Package ID: VL1209154-1

Date Printed: Wednesday, September 19, 2012 ALS Environmental -- FC

Page 1 of 2

LMs Version: 6.511 


\section{GC/MS Volatiles}

Method SW8260C

Matrix Spike And Matrix Spike Duplicate

Lab Name: ALS Environmental - FC

Work Order Number: 1209154

Client Name: Battelle Energy Alliance

ClientProject ID: MFC Biociesel Tank Removal ,TOS-A1175 BEA030488

\section{Surrogate Recovery MS/MSD}

\begin{tabular}{|l|l|c|c|c|c|c|c|}
\hline CASNO & \multicolumn{1}{|c|}{ Target Analyte } & $\begin{array}{c}\text { Spike } \\
\text { Added }\end{array}$ & $\begin{array}{c}\text { MS } \% \\
\text { Rec. }\end{array}$ & $\begin{array}{c}\text { MS } \\
\text { Flag }\end{array}$ & $\begin{array}{c}\text { MSD \% } \\
\text { Rec. }\end{array}$ & $\begin{array}{c}\text { MSD } \\
\text { Flag }\end{array}$ & $\begin{array}{c}\text { Control } \\
\text { Limits }\end{array}$ \\
\hline $460-00-4$ & 4-EROMOFLUOROBENZENE & 57 & $9 \theta$ & & 88 & & $52-151$ \\
\hline $1868-53-7$ & DIEROMOFLUOROMETHANE & 57 & 97 & & $9 \theta$ & & $61-134$ \\
\hline $2037-26-5$ & TOLUENE-D8 & 57 & $9 \theta$ & & $9 \theta$ & & $57-135$ \\
\hline
\end{tabular}




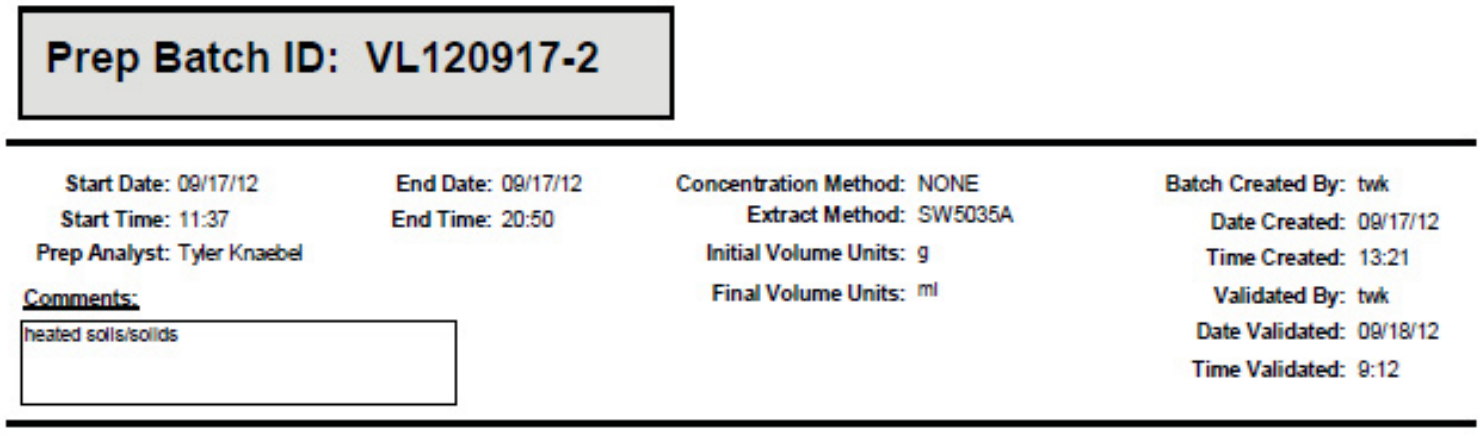

QC Batch ID: VL120917-2-2

\begin{tabular}{|c|c|c|c|c|c|c|c|c|c|}
\hline Lab ID & $\begin{array}{l}\text { QC } \\
\text { Type }\end{array}$ & Field ID & Matrix & $\begin{array}{c}\text { Date } \\
\text { Collected }\end{array}$ & $\begin{array}{l}\text { Initial } \\
\text { WtVol }\end{array}$ & $\begin{array}{l}\text { Final } \\
\text { Wt/Vol }\end{array}$ & $\begin{array}{l}\text { Cleanup } \\
\text { Method }\end{array}$ & $\begin{array}{c}\text { Cleanup } \\
\text { DF }\end{array}$ & $\begin{array}{l}\text { Order } \\
\text { Number }\end{array}$ \\
\hline VL120917-2 & $M B$ & $x \times 000 x$ & SOIL & $X X X C X X$ & 5 & 5 & NONE & 1 & 1209154 \\
\hline VL120917-2 & LCS & $x \times 000 x$ & SOIL & $X X X X X X$ & 5 & 5 & NONE & 1 & 1209154 \\
\hline VL12 $\overline{20917-2}$ & $\overline{L C S D}$ & $x \times 000 x$ & $\overline{\text { SOIL }}$ & $\overline{X X C D X X}$ & 5 & 5 & NONE & 1 & $\overline{12091} 54$ \\
\hline$1 2 0 \longdiv { 9 1 5 4 - 1 }$ & MS & BEAD30488_MFC & $\overline{\text { SOIL }}$ & $9 / 10 / 2012$ & 5 & 5 & NONE & 1 & $\overline{12091} 54$ \\
\hline$1 2 0 \longdiv { 9 1 5 4 - 1 }$ & MSD & BEAD30488_MFC & SOIL & $9 / 10 / 2012$ & 5 & 5 & NONE & 1 & $\overline{12091} 54$ \\
\hline$1 2 0 \longdiv { 9 1 5 4 - 1 }$ & SMP & BEAD30488_MFC & SOIL & $9 / 10 / 2012$ & 5 & 5 & NONE & 1 & $\overline{12091} 54$ \\
\hline$1 2 0 \longdiv { 9 1 5 4 - 1 2 }$ & SMP & BEAD30499_MFC & $\overline{\text { SOIL }}$ & $9 / 10 / 2012$ & 5 & 5 & NONE & 1 & $\overline{12091} 54$ \\
\hline$1 2 0 \longdiv { 9 1 5 4 - 4 }$ & SMP & BEAD30491_MFC & SOIL & $9 / 10 / 2012$ & 5 & 5 & NONE & 1 & $\overline{12091} 54$ \\
\hline $120 \overline{9154-5}$ & SMP & BEAD30492_MFC & $\overline{\text { SOIL }}$ & $9 / 10 / 2012$ & 5 & 5 & NONE & 1 & $\overline{12091} 54$ \\
\hline $120 \overline{154-8}$ & SMP & BEAD30495_MFC & $\overline{\text { SOIL }}$ & $9 / 10 / 2012$ & 5 & 5 & NONE & 1 & $\overline{1209154}$ \\
\hline $120 \overline{9154-9}$ & SMP & BEAD30496_MFC & SOIL & $9 / 10 / 2012$ & 5 & 5 & NONE & 1 & 1209154 \\
\hline
\end{tabular}

\section{QC. Ivpes}

\begin{tabular}{|l|l|}
\hline CAR & Carrer reference sample \\
\hline LCS & Laboratory Control Sample \\
\hline MB & Method Blank \\
\hline MSD & Laboratory Matrix Splke Dupllcate \\
\hline RVS & Reporting Level Veriflcation Standar \\
\hline SYS & Sample Yleld Splke \\
\hline
\end{tabular}

\begin{tabular}{|l|l|}
\hline DUP & Laboratory Duplicate \\
\hline LCSD & Laboratory Control Sample Duplicat \\
\hline MS & Laboratory Matrix Splke \\
\hline REP & Sample replicate \\
\hline SMP & Fleld Sample \\
\hline
\end{tabular}

srs Sample Yeld Splke 


\section{Volatile Organic GC/MS Tuning And Mass Calibration--Bromofluorobenzene (BFB)}

Lab Name: ALS Environmental - FC Work Order Number: 1209154

Client Name: Battelle Energy Alliance

ClientProject ID: BEA030488MFC Biodiesel Tank Removal ,TOS-A1175
BFB Injection Date: $\quad$ 7/16/2012 BFB Injection Time: $\quad 11: 31$

Instrument ID: $\quad$ HPV2

Reported on: Wednesday, September 19, 2012

Level: Low

Column: CAP

FilelD: $B 77985$

\begin{tabular}{|l|l|c|}
\hline mie & \multicolumn{1}{|l|}{ lon Abundance Criteria } & $\begin{array}{c}\text { \% Relative } \\
\text { Abundance }\end{array}$ \\
\hline 50 & $15.0-40.0$ percent of mass 95 & 22.5 \\
\hline 75 & $30.0-60.0$ percent of mass 95 & 51.5 \\
\hline 95 & Base peak, 100 percent of relative abundance & 100 \\
\hline 96 & $5.0-9.0$ percent of mass 85 & 6.8 \\
\hline 173 & Less than 20 percent of mass 174 & 0 \\
\hline 174 & Greater than 50.0 percent of mass 95 & 75.9 \\
\hline 175 & $5.0-9.0$ percent of mass 174 & 7.1 \\
\hline 176 & Greater than 95.0 percent $<101.0$ percent of mass 174 & 96.9 \\
\hline 177 & $5.0-9.0$ percent of mass 176 & 6.5 \\
\hline
\end{tabular}

THIS TUNE APPLIES TO THE FOLLOWING SAMPLES, MS/MSD, BLANKS, AND STANDARDS

\begin{tabular}{|c|c|c|c|c|c|}
\hline Client Sample ID & Lab Sample ID & Lab File ID & $\begin{array}{c}\text { Date } \\
\text { Analyzed }\end{array}$ & $\begin{array}{c}\text { Time } \\
\text { Analyzed }\end{array}$ & QC BatchID \\
\hline$x 000000 x$ & VOC_2ppb_ICALCSTD & B77998 & $7 / 16 / 2012$ & $12: 30$ & VL120716-2A \\
\hline$x 000000 X$ & VOC_4ppb_ICALCSTD & B77999 & $7 / 16 / 2012$ & 12.52 & VL120716-2A \\
\hline$x 000000 x$ & VOC_10ppb_ICALCSTD & B78000 & $7 / 16 / 2012$ & $13: 14$ & VL120716-2A \\
\hline$x \times 10000 x$ & VOC_20ppb_ICALCSTD & B78001 & $7 / 16 / 2012$ & $13: 35$ & VL120716-2A \\
\hline$x \times x 000 x$ & VOC_40ppb_ICALCSTD & B78002 & $7 / 16 / 2012$ & 13.57 & VL120716-2A \\
\hline$x 000000 X$ & VOC_60ppb_ICALCSTD & $\mathrm{B} 78003$ & $7 / 16 / 2012$ & $14: 19$ & VL120716-2A \\
\hline$x 000000 x$ & VOC_80ppb_ICALCSTD & B78004 & $7 / 16 / 2012$ & $14: 41$ & VL120716-2A \\
\hline 10000000 & VOC_120ppb_ICALCSTD & B78005 & $7 / 16 / 2012$ & $15: 03$ & VL120716-2A \\
\hline$x 000000 x$ & VOC_160ppb_ICALCSTD & B78006 & $7 / 16 / 2012$ & $15: 25$ & VL120716-2A \\
\hline$x 00000 x$ & VL120716-2ICVICV & B78008 & $7 / 16 / 2012$ & $16: 08$ & VL120716-2A \\
\hline$x 000000 x$ & VL120716-2LCS & B78009 & $7 / 16 / 2012$ & $16: 31$ & VL120716-2-1 \\
\hline$x 000000 x$ & VL120716-2LCSD & B78011 & $7 / 16 / 2012$ & $17: 27$ & VL120716-2-1 \\
\hline$x 000000 x$ & VL120716-2MB & B78012 & $7 / 16 / 2012$ & $17: 49$ & VL120716-2-1 \\
\hline$x 000000 x$ & $1207118-1$ & B78013 & $7 / 16 / 2012$ & $18: 10$ & VL120716-2-1 \\
\hline$x \times X X 00 X X$ & 1207118-1DUP & B78014 & $7 / 16 / 2012$ & $18: 32$ & VL120716-2-1 \\
\hline$x 0 \times 0000 X$ & 1207118-1MS & B78015 & $7 / 16 / 2012$ & 18.53 & VL120716-2-1 \\
\hline$x 000000 X$ & $1207118-2$ & B78016 & $7 / 16 / 2012$ & $19: 15$ & VL120716-2-1 \\
\hline
\end{tabular}

Data Package ID: VL1209154-1

Date Printed: Wednesday, September 19, $2012 \quad \begin{array}{r}\text { ALS Environmental - FC } \\ \text { Lus verion: 6.611 }\end{array}$

Page 1 of 2

26 of 36 


\section{$5 A$}

Volatile Organic GC/MS Tuning And Mass

Calibration--Bromofluorobenzene (BFB)

Lab Name: ALS Environmental - FC

BFB Injection Date: $\quad$ 8/17/2012

BFB Injection Time: $\quad$ 11:37

Instrument ID: HPV/2

Client Name: Battelle Energy Alliance

ClientProject ID: BEA030488MFC Biodiesel Tank Removal ,TOS-A1175

Reported on: Wednesday, September 19, 2012
Level: Low
Column: CAP
FilelD: $\mathbf{B} 79249$

\begin{tabular}{|l|l|c|}
\hline mie & \multicolumn{1}{|c|}{$\begin{array}{c}\text { lon Abundance Criteria } \\
\text { SW8260C }\end{array}$} & $\begin{array}{c}\text { \% Relative } \\
\text { Abundance }\end{array}$ \\
\hline 50 & $15.0-40.0$ percent of mass 95 & 21.6 \\
\hline 75 & $30.0-60.0$ percent of mass 95 & 49.3 \\
\hline 95 & Base peak, 100 percent of relative abundance & 100 \\
\hline 96 & $5.0-8.0$ percent of mass 95 & 6.7 \\
\hline 173 & Less than 2.0 percent of mass 174 & 0 \\
\hline 174 & Greater than 50.0 percent of mass 95 & 78.2 \\
\hline 175 & $5.0-9.0$ percent of mass 174 & 6.8 \\
\hline 176 & Greater than 95.0 percent $<101.0$ percent of mass 174 & 99 \\
\hline 177 & $5.0-9.0$ percent of mass 176 & 6.2 \\
\hline
\end{tabular}

THIS TUNE APPLIES TO THE FOLLOWING SAMPLES, MS/MSD, BLANKS, AND STANDARDS:

\begin{tabular}{|c|c|c|c|c|c|}
\hline Client Sample ID & Lab Sample ID & Lab File ID & $\begin{array}{c}\text { Date } \\
\text { Analyzed }\end{array}$ & $\begin{array}{c}\text { Time } \\
\text { Analyzed }\end{array}$ & QC BatchlD \\
\hline$x \times C O O C X X$ & VL120917-2RVS & B79251 & 8/17/2012 & $12: 15$ & VL120917-2-3 \\
\hline$x 000000 X$ & $\mathrm{CCV} 1 \mathrm{CCV}$ & B79252 & 9/17/2012 & $12: 39$ & VL120917-2A \\
\hline$x 000000 x$ & VL120917-2LCS & B79253 & $\mathrm{Q} / 17 / 2012$ & $13: 03$ & VL120917-2-1 \\
\hline$x 0 \times 000 X X$ & VL120917-2LCS & B79253 & 8/17/2012 & $13: 03$ & VL120917-2-2 \\
\hline$x 000000 x$ & VL120917-2LCSD & B79254 & $8 / 17 / 2012$ & $13: 27$ & VL120917-2-1 \\
\hline$x 000000 x$ & VL120917-2LCSD & B79254 & $8 / 17 / 2012$ & $13: 27$ & VL120917-2-2 \\
\hline $0000000 x$ & VL120917-2MB & $\mathrm{B} 79255$ & $9 / 17 / 2012$ & 13.51 & VL120917-2-1 \\
\hline$x 000000 x$ & VL120917-2MB & $\mathrm{B} 79255$ & $8 / 17 / 2012$ & $13-51$ & VL120917-2-2 \\
\hline BEAD30488_MFC & $1200154-1$ & $\mathrm{~B} 79256$ & $8 / 17 / 2012$ & $14: 15$ & VL120917-2-2 \\
\hline BEAD30491_MFC & $1209154-4$ & $\mathrm{~B} 79257$ & $8 / 17 / 2012$ & $14: 39$ & VL120917-2-2 \\
\hline BEAD30492_MFC & $1209154-5$ & $\mathrm{~B} 79258$ & $8 / 17 / 2012$ & $15: 01$ & VL120917-2-2 \\
\hline BEA030495_MFC & $1209154-8$ & B79250 & $8 / 17 / 2012$ & $15: 22$ & VL120917-2-2 \\
\hline BEAD30496_MFC & $1209154-9$ & B79260 & $8 / 17 / 2012$ & $15: 44$ & VL120917-2-2 \\
\hline BEAD30499_MFC & $1209154-12$ & B79261 & $9 / 17 / 2012$ & $16: 08$ & VL120917-2-2 \\
\hline BEA030488_MFC & $1209154-1 \mathrm{MS}$ & $\mathrm{B} 79262$ & $9 / 17 / 2012$ & $16: 29$ & VL120917-2-2 \\
\hline BEAD30488_MFC & 1200154-1MSD & $\mathrm{B} 79263$ & $9 / 17 / 2012$ & $16-51$ & VL120917-2-2 \\
\hline$x 0 X 000 x$ & $1209093-2$ & B79264 & 8/17/2012 & $17: 13$ & VL120917-2-1 \\
\hline
\end{tabular}

Data Package ID: VL1209154-1

\section{Date Printed: Wednesday, Septenber 19,2012}

Date Printed: Wednesday, September 19, 2012
ALS Environmental - FC

LIMS Verion: 6.611
Page 1 of 2

27 of 36 
Gaubration IU: UI IO i<0

Instrument ID: HPV/2

Calibration Date: 7/16/2012
MLU டIIVIUUIIICIILI -- I Initial Calibration Report

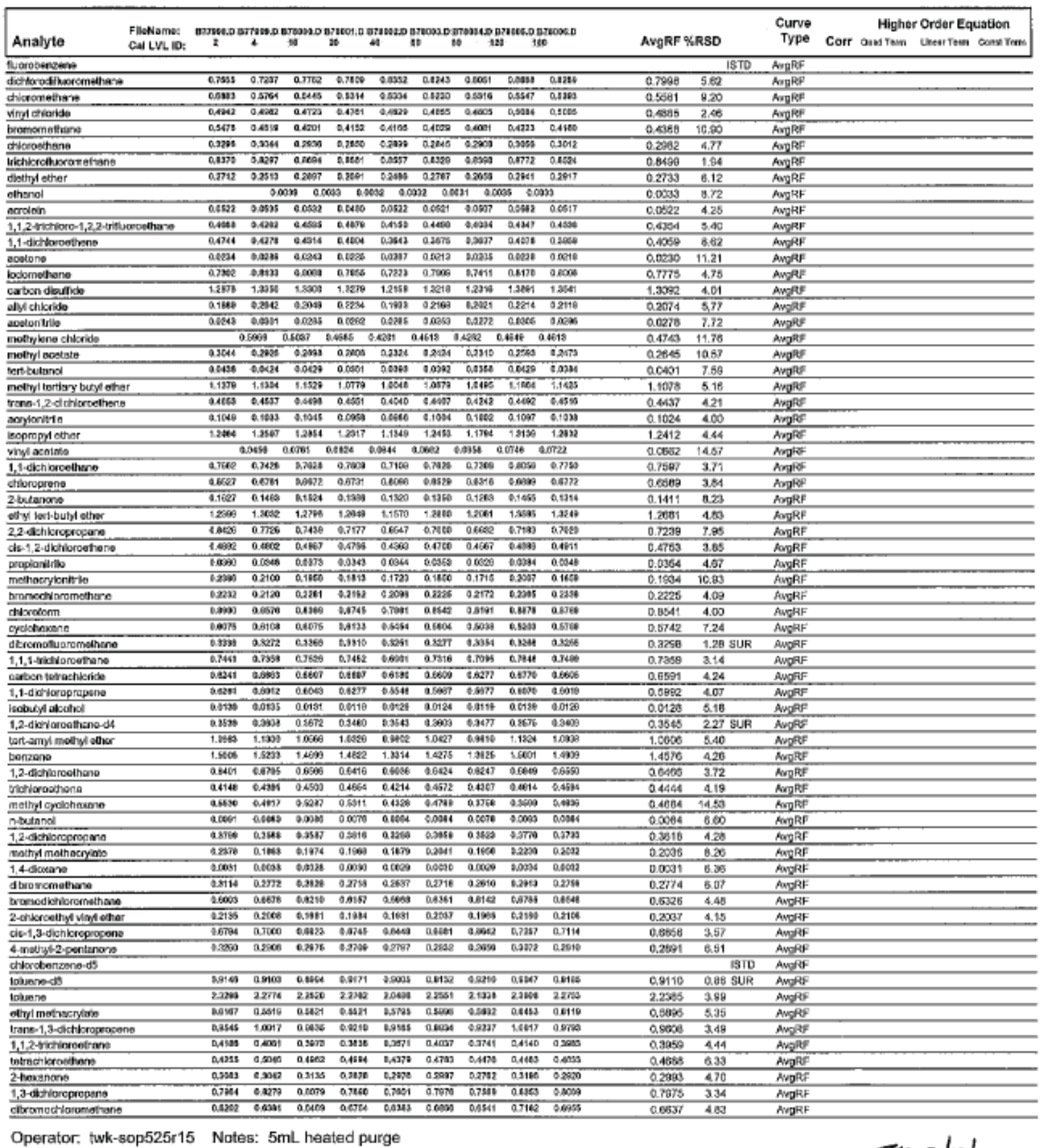

Operator. twk-sop525r15 Notes: $5 \mathrm{~mL}$ heated purge

Date Printed: Tueeday, July 17, 2012

ALS Environmental -- FC

Page 1 of 2

LMB8 Verrion: 6,800

28 of 36 


\begin{tabular}{|c|c|c|c|c|c|c|c|c|c|c|c|c|c|c|}
\hline \multicolumn{4}{|c|}{$\begin{array}{l}\text { Calibration ID: } 071612 \mathrm{~S} \\
\text { Instrument ID: HPV2 } \\
\text { Calibration Date: } 7 / 16 / 2012\end{array}$} & \multicolumn{8}{|c|}{ Initial Calibration Report } & \multirow[b]{2}{*}{$\begin{array}{c}\text { Curve } \\
\text { Type }\end{array}$} & \multirow{2}{*}{ Conr } & \multirow{2}{*}{$\begin{array}{l}\text { Higher Order Equation } \\
\text { Owe Tros Lhax Tom cerst Toue }\end{array}$} \\
\hline \multirow{2}{*}{ Analyte } & \multirow{2}{*}{\multicolumn{3}{|c|}{ 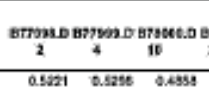 }} & \multirow{2}{*}{$\frac{0.5196}{20}$} & \multirow{2}{*}{ 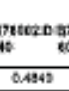 } & \multirow{2}{*}{ 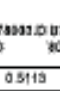 } & \multicolumn{3}{|c|}{ 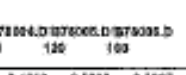 } & \multicolumn{2}{|c|}{ AvgRF \%RSD } & & & \\
\hline & & & & & & & 0.4913 & 0.593 & कडset & 0.5009 & 3.90 & AargF & & \\
\hline 1. chilerohockane & aster & 0.811 & 0.3678 & 0.Esio & 0.754 & 0.5070 & 0.8613 & 0.8247 & 0.0565 & 0.7581 & 10.84 & Aovgr & & \\
\hline chlombercesne & LAEE2 & 1.A685 & I.min & 1.4Tü & 1.5013 & IAS15 & $1,3 \mathrm{mat}$ & 1,274 & 1,485 & 1,4538 & 2.90 & Awpraf & & \\
\hline ellygternzone & 25063 & $2.685 y$ & 2.5522 & 26310 & 2.3594 & 26000 & 2.3532 & 24514 & 28416 & 2.5502 & 4.67 & AngRF & & \\
\hline 1,1,1,2-sotrachilar zolthenese & o.segr & 0.5062 & 0.5255 & 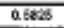 & 0.5365 & 0.5030 & 0.5627 & 0.5876 & OBESG & 0,6740 & 3,57 & AngRF & & \\
\hline entp-xylene & $0.0=20$ & 0.6050 & 0.2227 & 0.0000 & 0.4458 & $9.02 x$ & 0.361 & 0.8898 & a.9244 & 0,8950 & 4.02 & AM:AF & & \\
\hline axplare & $260 \mathrm{es}$ & 0.0656 & 0.02200 & acois & 0.5342 & 0.906 & 0,5202 & 0.858 & 9.5012 & 0.8855 & 3.75 & Amgaf & & \\
\hline stymen & $1.57 \pi$ & 1.5510 & 1.3921 & 1.5781 & 1,4412 & 1.5119 & 9,4202 & 1.5294 & 10174 & 1.5504 & $3 \mathrm{MH}$ & AWgRF & & \\
\hline reanotorm & 0.2684 & 0.2724 & 0.37 & 0.3201 & 0.323 & 0,005 & 8,2954 & 0.4330 & $0,1,20$ & 0.3944 & 5.01 & AnSPF & & \\
\hline sepmppleanpane & 22364 & 2.3002 & 2.3438 & $2.36 \mathrm{~m}$ & 2,1372 & 2.394 & $9, a m 7$ & thate & $22 x+50$ & 22419 & 7.50 & AW:PF & & \\
\hline 4tbramofiarabonzzana & करण & 0.4601 & 0.408 & 0.4138 & 0.422 & (.,20030 & 0.4105 & 0.4140 & 9.4.192 & 0.4009 & 1.21 SUR & ANopF & & \\
\hline 1.4-dkhibrobaravena-d6 & & & & & & & & & & & ISTO & AWOPF & & \\
\hline 1,1,2-2-totrachlorsethane & 10004 & 1.075 & 1.0440 & 1000 & 1.062 & 1.93n & 0.0811 & 2.0060 & 1.02000 & 1.0446 & 3.61 & ANgPF & & \\
\hline Hpropybanzano & 1.7421 & 6.1647 & 5.7964 & acees & 5.2449 & 1.9502 & 4.7311 & 4.5551 & $4 ., 552$ & 5.6205 & 1022 & AwgrF & & \\
\hline 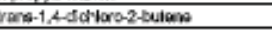 & 0.4002 & $0.8 c e 0$ & 0.354 & 0.5000 & 0.5250 & 6.3459 & 0.1122 & 0.2587 & 0.3125 & 0.3317 & 245 & AngPS & & \\
\hline 1,2,3-tichibrcpropenen & 0.5007 & $a .5850$ & 0.3560 & 0.555 & 0.0250 & 0.030 & 0.110 & 0.060 & 6330 & 0.3481 & 480 & ANgPF & & \\
\hline Gomobinearse & का & $1.8 E 15$ & 1.1006 & 12654 & 1.1785 & 1.2562 & 1,1536 & 12100 & 12349 & 1.2210 & $3 \pi$ & Angpe & & \\
\hline 1,3,5-5imedtrylberzene & 29261 & 40000 & 3.9880 & 80005 & sent & 2.3790 & $24 m$ & 1.1300 & 2.9152 & 37340 & 2.58 & Angpe & & \\
\hline 2-athrotokana & $\cos 16$ & 1.0000 & 1,0061 & 0.1212 & 1.0184 & 1.0148 & 0.0450 & 0.053 & 1.40282 & 1.0319 & 5,98 & Avgafe & & \\
\hline sedibretgibane & 10535 & 1.1002 & 1.9720 & 1,0583 & $1.04 \sqrt{2}$ & 1.1220 & L.,2858 & 0.9705 & 9.9493 & 1.0008 & 8.78 & Angrof & & \\
\hline lect-budyeanzane & 4.9221 & arol7 & $0.7 \pm 4$ & 0.7834 & nasper & 0.738 & $0.8 E 1$ & 0.034 & 0.0584 & 0,6979 & 1921 & Angfo & & \\
\hline 12.4-rimatirytanzane & 2.9433 & 2.070 & 2.221 & 3.9212 & 19417 & 3.3108 & 21207 & 2005 & $2 . \sin 7$ & 2 ec593 & 270 & Axgif: & & \\
\hline 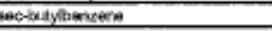 & 4.9238 & 4073 & $5,04 \pi 3$ & S, Sונז & 12004 & 4.7152 & 96342 & 1.001 & 4.3513 & 44992 & 1991 & Aorly & & \\
\hline p-thogragsilolument & 2.0344 & 4005 & 4.911 & 4.151 & 24005 & 2.0742 & 2.0052 & 27202 & 2.5125 & 9.8112 & 19.90 & Aorper & & \\
\hline 1.5-dectikrctennzen & 2.203 & 222003 & 2.1460 & 22825 & 2120 & 2.2112 & 1606 & 1921 & 24114 & 2.1939 & 6.60 & Aonfs: & & \\
\hline 1,4det kerdenenaw & 2.1294 & 22005 & 2,1258 & 2,1054 & 2,1140 & 2238 & 1.5011 & 1,208 & 2,0584 & 2.1004 & 5.70 & fouRF & & \\
\hline n-tublegbenzana & 3,969 & 4.0735 & S,OEA & A,0781 & 13342 & 3,4185 & 280772 & $2 \operatorname{san}$ & $243+1$ & 3.5678 & 15.43 & foogR & & \\
\hline 1,2-tidtilenchenzans & 2,0212 & sams & 1,509 & 2,094 & 1909 & 20104 & 1,7002 & 1.910 & 1.6740 & 1.0036 & 5.12 & AowRF & & \\
\hline hesusctibreothen & 0.5228 & anov7 & 0.0352 & 2,0194 & 0.000 & aet78 & 0,1006 & B.4487 & ateis & 0.5571 & 12.35 & $A D R F$ & & \\
\hline 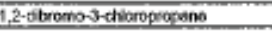 & Q.1050 & 0.251 & 0.2016 & D,th: & वसका & asec & 0.1000 & 0.2221 & as10 & 0.2001 & 6.61 & AusRF & & \\
\hline 1,2,4-andiveruberizens & 1230 & $13 \mathrm{in}$ & 1.3281 & 9,2967 & सक्षा & 1.3180 & 1000 & 6.0205 & 1.1617 & 7.2129 & 11.97 & Avelif & & \\
\hline hewacticrobutsdsne & 0.929 & 0.5015 & 0.679 & D.SWI! & 0.8972 & $0 \times 2070$ & $0,0,000$ & 8.3012 & aesco & 0.4006 & 17.45 & AughF & & \\
\hline 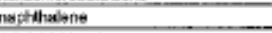 & 2430 & 2.5597 & 2.060 & 2.447 & $2 \operatorname{sos} 3$ & $2 E 21$ & 2.1729 & 2.285 & 2518 & 24006 & 5.50 & Anger & & \\
\hline 1,2,3tiktiverubentamis & 10022 & 1,1207 & 1.2076 & 9,4470 & 10042 & 1.1622 & 0.911 & काM7 & $19 \pi$ & 1.0756 & 11.88 & Anpla & & \\
\hline 2-athytraxanuel & & & & & & & & & & & & & & \\
\hline
\end{tabular}

Average RSD $=6.40$

Concentration Multipliers

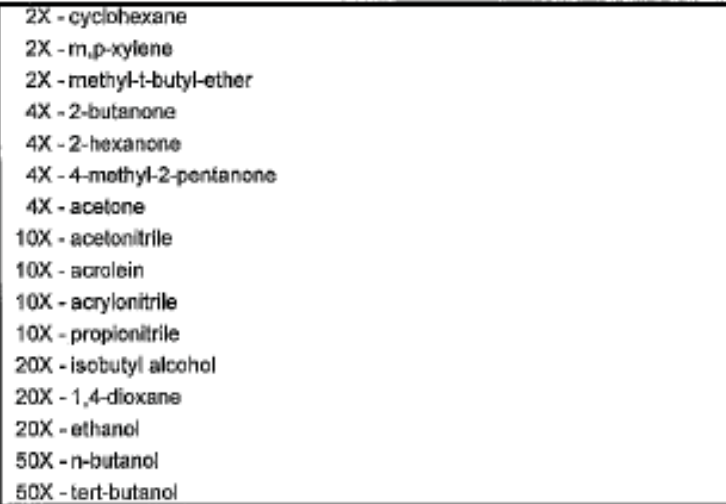

Operator: hwk-8op525r15 Notes: $5 \mathrm{~mL}$ heated purge

Date Printed:Tuesday, July 17, 2012
ALS Environmental - FC

L.MS Vursion: 6.009 $\operatorname{min/13}$

Page 2 of 2

29 of 36 


\section{ALS Environmental -- FC}

\section{Initial Calibration Verification}

\begin{tabular}{|cc|}
\hline Lab Sample ID: VL120716-2ICV & Calibration ID: $071612 \mathrm{~S}$ \\
\hline Analysis Date: $7 / 16 / 2012$ & Instrument ID: $\mathrm{HPV} 2$ \\
\hline File Name: B78008 & Calibration Date: $7 / 16 / 2012$ \\
\hline
\end{tabular}

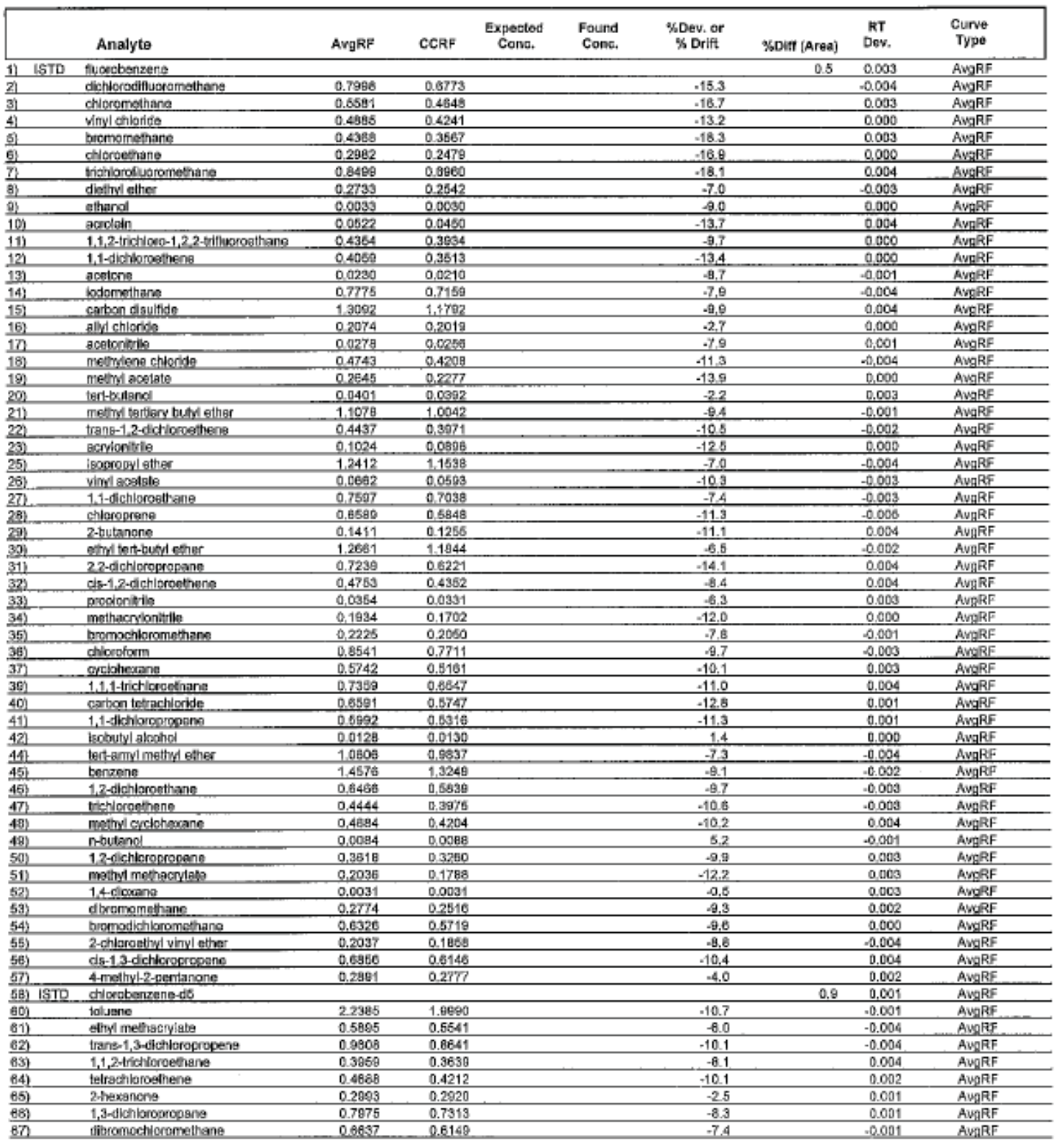

Operator: twik-sop52.5r-15

$\pi+1,7 / 2=$

Date Printed: Tuesday, July 17, 2012

ALS Environmental -- FC

LMAs versione 6,8009

Page 30 of 2 or 36 


\section{ALS Environmental -- FC}

\section{Initial Calibration Verification}

\begin{tabular}{|cc|}
\hline Lab Sample ID: VL120716-2ICV & Calibration ID: $071612 S$ \\
\hline Analysis Date: $7 / 16 / 2012$ & Instrument ID: HPV2 \\
File Name: $B 78008$ & Calibration Date: $7 / 16 / 2012$ \\
\hline
\end{tabular}

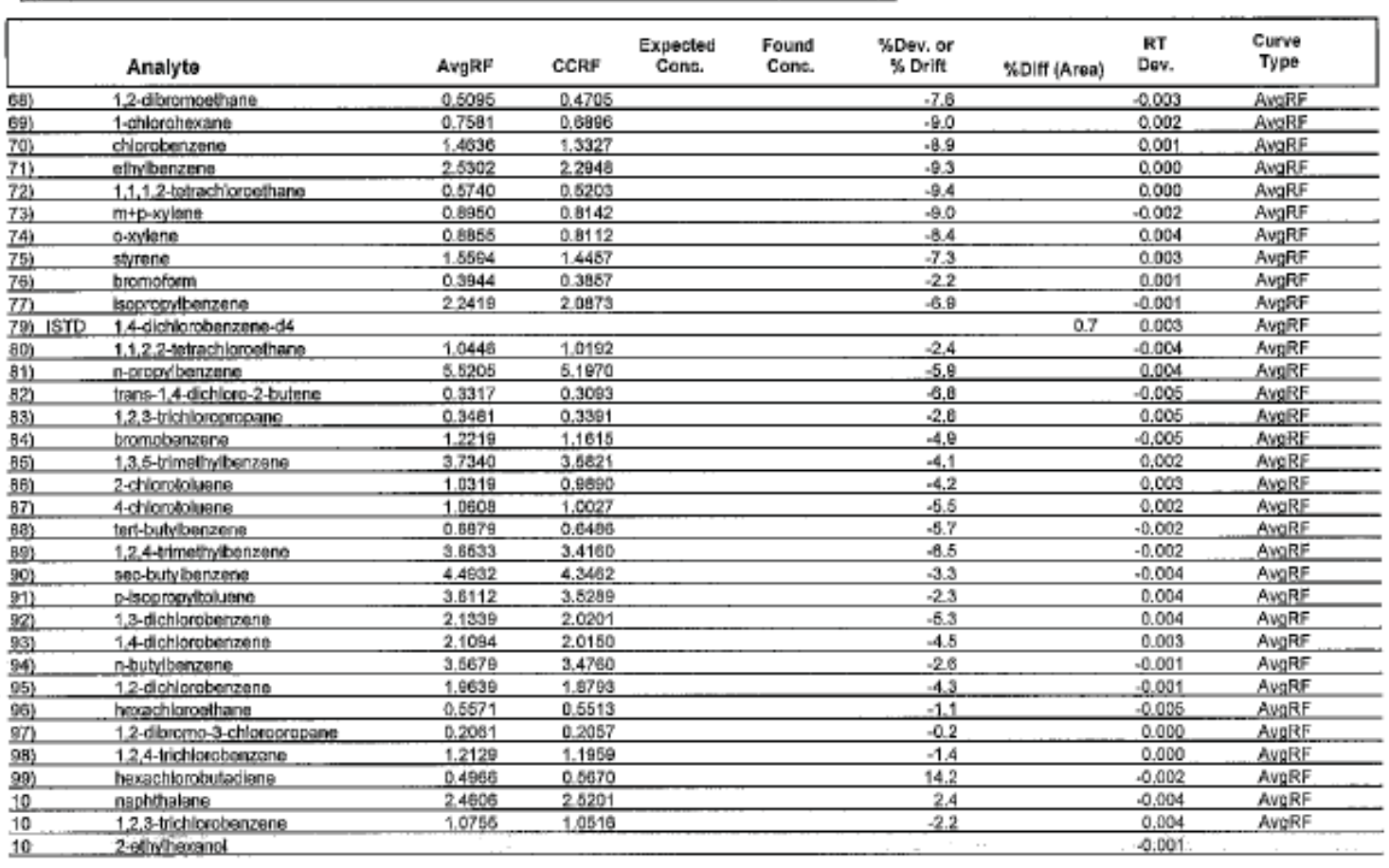




\section{ALS Environmental -- FC \\ Continuing Calibration Verification}

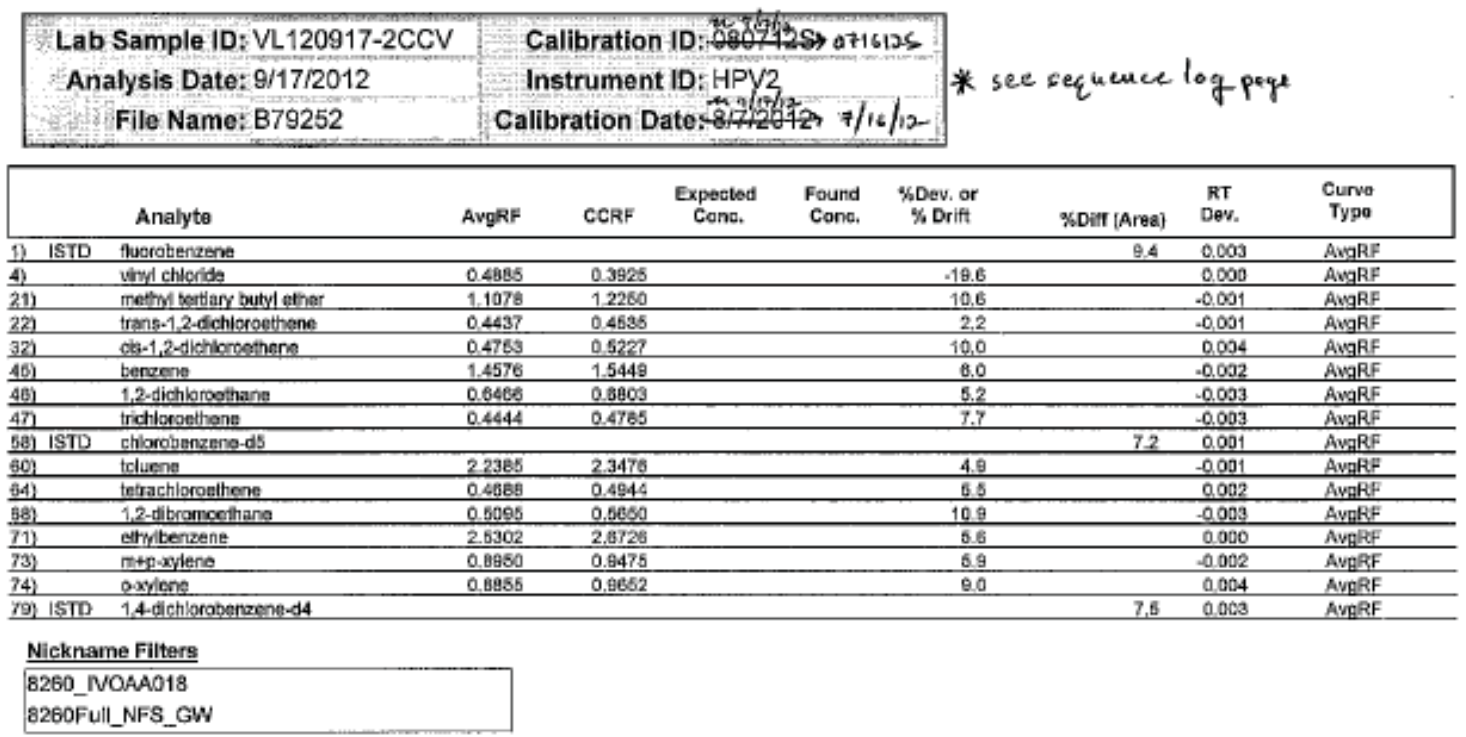




\section{$8 \mathrm{~A}$}

Volatile Internal Standard Area Summary

Lab Name: ALS Environmental - FC

Date Analyzed:

$9 / 17 / 2012$

Work Order Number: 1209154

Time Analyzed: $\quad 12: 38$

Client Name: Battelle Energy Alliance

ClientProject ID: BEA030488 MFC Biodiesel Tank Remova ,TOS-A1175

Reported on: Wednesday, September 19, 2012

Instrument ID: HPV2

Lab File ID: $\quad$ B79252

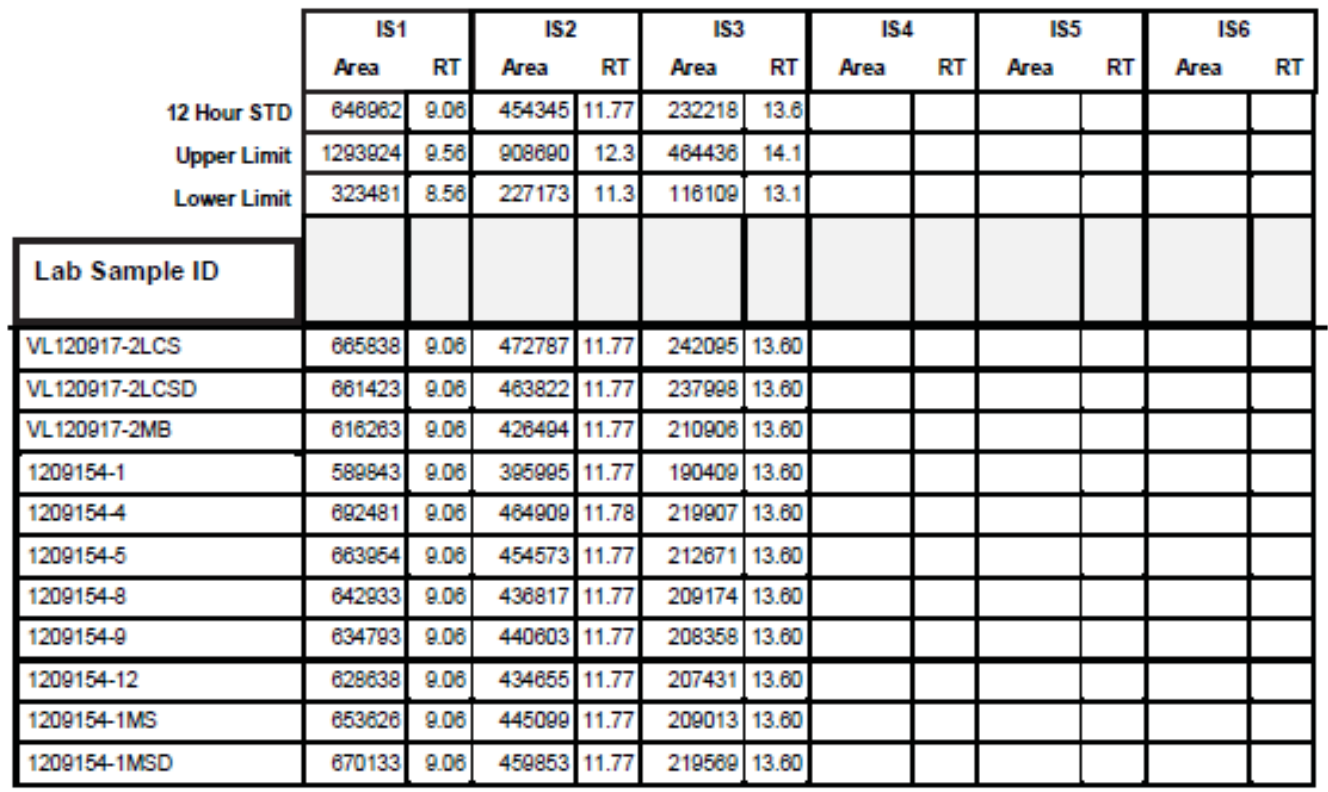

Shaded values exceed established area count limits.

LMS Veralon: 6.511

Upper Limit $=+100$ percent of internal standard area.

Lower Limit $=-50$ percent of internal standard area.

33 of 36 


\section{Ats}

\section{Supporting Raw Data}




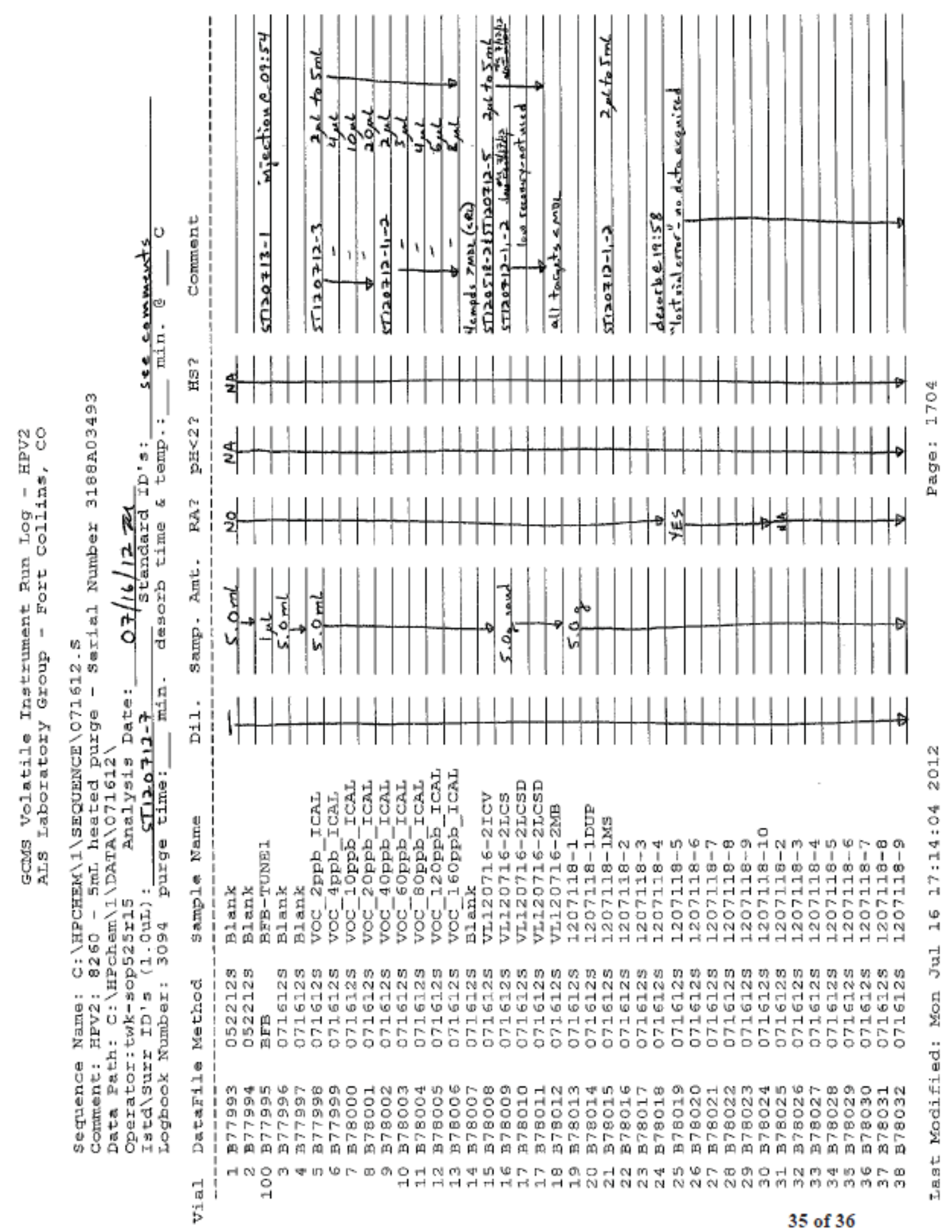



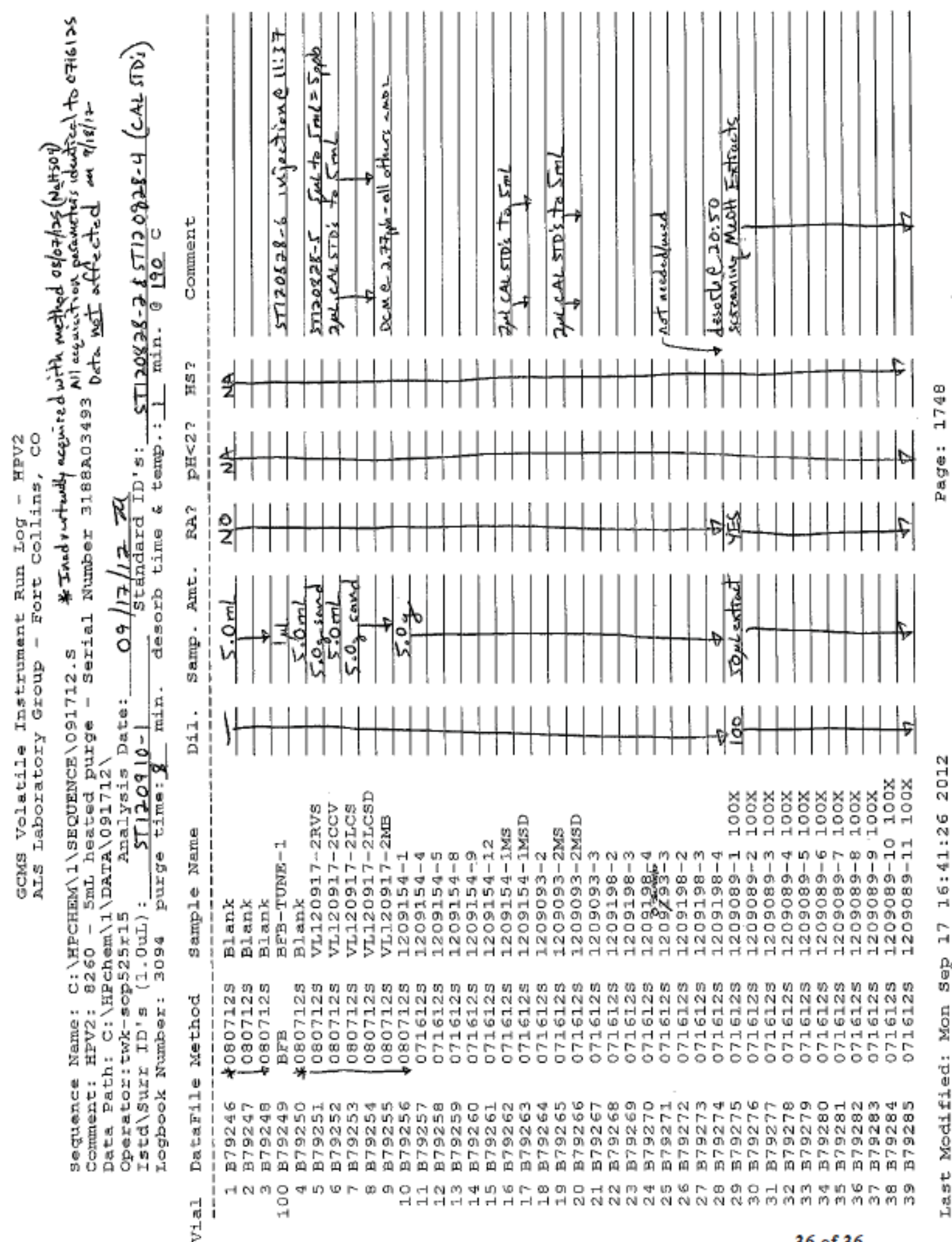

36 of 36 


\title{
Appendix E \\ DEQ Notification of Sample Results for MFC UST Closure (CCN 228521)
}

\author{
Idaho National Laboratory Mail - DEQ notification of sample results for ... hattps:/mail.google.com/mail/b/277/u/0/7ui=2\&ik=2le29e2ce5\&view=p...
}

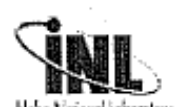

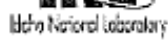

$\operatorname{CCN} 228521$

ServiceID, ENVIRONMENTAL CORRESPONDENCE <envaff@inl.gov>

DEQ notification of sample results for the MFC UST closure

1 message

Griffith, Bradley $\mathrm{K}$ <bradley.griffith@inl.gov>

Thu, Sep 20, 2012 at 4:29 PM

To: ENVIRONMENTAL CORRESPONDENCE ServicelD <envaff@inl.gov>, James F Graham <james.graham@inl.gov>,

Timothy L Carlson <timothy.carlson@inl.gov>, Timothy A Miller <timothy.miller@inl.gov>

On Thursday, Sept. 20th at approximately 9:00 AM I called Steve Heaton (Idaho DEQ) regarding sample results that were received at approximately 3:30 PM on Sept. 19th for the MFC Biodiesel UST closure. Jason Sturm (DOE) was also on the call. I left a message on Mr. Heaton's voicemall stating some of the sample results were above the residential use screening levels and asked for him to return my call. At approximately 10:00 AM Mr. Heaton returned my call and I gave him details about the sample results, went over options such as removing more soil and sampling again, or completing a site specific risk evaluation. Mr. Heaton thought removing additional soll and sampling may bring us to the desired screening levels. I also told Mr. Heaton that the phone call should be considered the required 24-hour notification. Mr. Heaton agreed and said he would log it when he returned to his office Monday, Sept. 24th.
\end{abstract}




\section{Appendix F \\ Sample Analytical Report \#2}

\section{Ats}

September 25, 2012

Ms. Peggy Scherbinske Battelle Energy Alliance

$2525 \mathrm{~N}$. Fremont Ave.

Idaho Falls, ID 83415-6194

$\begin{array}{lll}\text { Re: } & \text { ALS Workorder: } & \text { 12-09-339 } \\ \text { Project Name: } & \text { MFC Biodiesel Tank Removal, TOS-A1175 Rev2 } \\ \text { Project Number: } & \text { BEA031231 }\end{array}$

Dear Ms. Scherbinske:

Eighteen soll samples were received from Battelle Energy Alliance on September 21, 2012. The samples were scheduled for the following analyses:

$\begin{array}{ll}\text { GC/MS Volatiles } & \text { pages 1-40 } \\ \text { GC/MS Semivolatiles } & \text { pages 1-47 }\end{array}$

The results for these analyses are contained in the enclosed report.

Thank you for your confidence in ALS Environmental. Should you have any questions, please call.

Sincerely,

1013 in

ALS Environmental

Jeff Kujawa

Project Manager

JRKerl

Enclosure (s): Report

ADDRESS 225 Commerce Drtve, Fort Collins, Colorado, USA 80S24 i PHONE +1970 4901511 ) FAX +1 9704901522 ALS GrOUP USA, COnP, Part of the ALS Laboratory Group A Campbell Brothers Limilted Conapany 
ALS Is accredited by the following accreditation bodies for various testing scopes in accordance with requirements of each accreditation body. All testing is performed under the laboratary management system, which is maintained to meet these requirement and regulations. Please contact the laboratory or atcreditation body for the current scope testing parameters.

\begin{tabular}{|c|c|}
\hline Wastington & $\mathrm{C} 1280$ \\
\hline Utah & Co00078 \\
\hline Arlzona & AZ0742 \\
\hline Alaska & UST-086 \\
\hline Alaska & Co00078 \\
\hline Flarida & E87914. \\
\hline Missouti & 175 \\
\hline North Dakota & $\mathrm{R}-057$ \\
\hline New Jersey & $\mathrm{COOO3}$ \\
\hline Nevada & $00000782008 \mathrm{~A}$ \\
\hline Calffornia & $06251 \mathrm{CA}$ \\
\hline Kansas & $E-10381$ \\
\hline Maryland & 285 \\
\hline Pennsylvania & $68-03116$ \\
\hline Texas & T104704241-09-1 \\
\hline Colorado & C000078 \\
\hline Connecticut & $\mathrm{PH}-0232$ \\
\hline Idaho & $\mathrm{CO00078}$ \\
\hline Tennessee & 2976 \\
\hline Kentucky & 90137 \\
\hline L-A-B (DoD ELAP/ISO 17025) & L2257 \\
\hline
\end{tabular}




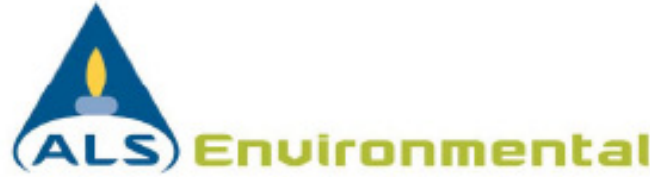

\section{GC/MS Semivolatiles SIMPAH}

\author{
Case Narrative
}

\section{Battelle Energy Alliance \\ MFC Biodiesel Tank Removal, TOS-A1175 Rev 2 -- BEA031231}

Work Order Number: 1209339

1. This report consists of 9 soil samples. The samples were received cool and intact by ALS on $09 / 21 / 12$.

2. These samples were prepared and analyzed according to SW-846, 3rd Edition procedures. Specifically, the soil samples were extracted using soxhlet procedures according to SW-846 Method $3540 \mathrm{C}$ utilizing the current revision of SOP 625 .

3. The extracts were analyzed using GC/MS with a DB-5MS capillary column according to the current revision of SOP 506 based on SW-846 Method 8270D. The samples were analyzed using selective ion monitoring (SIM), in order to achieve lower reporting limits. All positive results were quantitated against the initial calibration standards using the intemal standard technique. The identification of positive results was achieved by a comparison of the retention time and a limited number of major ions from the mass spectrum of the sample versus the daily calibration standard.

4. All initial calibration criteria were met. If average response factors were used in the initial calibration, \%RSD was $\leq 20 \%$. If linear or higher order regression calibrations were used in the initial calibration, the coefficient of determination $\left(r^{2}\right) \geq 0.99$.

5. All initial calibration standards are verified by comparing a second source standard initial calibration verification (ICV) against the calibration curve. All target compounds in the second source verification had a $\% \mathrm{D} \leq 30 \%$.

6. All method blank criteria were met.

7. All laboratory control sample and laboratory control sample duplicate recoveries and RPDs were within the acceptance criteria. 


\section{Ats}

8. Sample $1209339-2$ was designated as the quality control sample for this analysis. Similarity of matrix and therefore relevance of the $Q C$ results should not be automatically inferred for any sample other than the native sample selected for QC.

All matrix spike and matrix spike duplicate recoveries and RPDs were within acceptance criteria with the following exceptions:

\begin{tabular}{l|c|c} 
Spiked Compound & QC Sample & Directión \\
\hline Several Compounds & MS/MSD & Low
\end{tabular}

The recoveries of these compounds in the laboratory control sample and laboratory control sample duplicate were within control limits, which suggest the outliers in the matrix spikes may have been due to matrix effects, so no further action was taken.

9. The samples were extracted and analyzed within the established holding times.

10. All surrogate recoveries were within acceptance criteria.

11. All internal standard recoveries were within acceptance criteria with the following exceptions:

\begin{tabular}{|c|c|c|}
\hline Internal Standard & Sample & Direction \\
\hline Perylene- $D_{12}$ & $1209339-2,-2 M S,-2 M S D$ & Low \\
& $-6,-9,-12,-14 \&-18$ & \\
\hline
\end{tabular}

Re-analysis of the samples duplicated the original result. This suggests that the outiliers were due to matrix effects. No further action was taken.

12. Sample 1209339-16 was analyzed at dilution to bring target analytes in to calibration range. The reporting limits have been adjusted accordingly.

13. Manual integrations are performed when needed to provide consistent and defensible data following the guidelines in the current revision of SOP 939.

The data contained in the following report have been reviewed and approved by the personnel listed below. In addition, ALS certifies that the analyses reported herein are true, complete and correct within the limits of the methods employed.

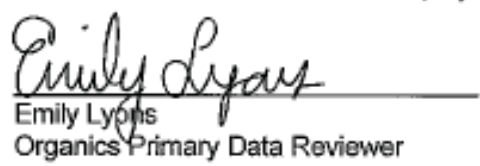

$\frac{\text { Ty h the hat }}{\text { Organics Final Data Reviewer }}$

$$
\frac{9-25-12}{\text { Date }}
$$


ALS

Data Qualifier Flags

Chromatography and Mass Spectrometry

U or ND: This flag indicates that the compound was analyzed for but not detected.

J: $\quad$ This flag indicates an estimated value. This flag is used as follows: (1) when estimating a concentration for tentatively identified compounds (TICs) where a 1:1 response is assumed; (2) when the mass spectral and retention time data indicate the presence of a compound that meets the volatile and semivolatile GC/MS identification criteria, and the result is less than the reporting limit (RL) but greater than the method detection limit (MDL); (3) when the retention time data indicate the presence of a compound that meets the GC identification criteria, and the result is less than the RL but greater than the MDL; and (4) the reported value is estimated.

B: $\quad$ This flag is used when the analyte is detected in the associated method blank as well as in the sample. It indicates probable blank contamination and warns the data user. This flag shall be used for a tentatively identified compound (TIC) as well as for a positively identified target compound.

E: This flag identifies compounds whose concentration exceeds the upper level of the calibration range.

A: This flag indicates that a tentatively identified compound is a suspected aldol-condensation product.

$\mathrm{X}$ : This flag indicates that the analyte was diluted below an accurate quantitation level.

*: This flag indicates that a spike recovery is equal to or outside the control criteria used.

+: $\quad$ This flag indicates that the relative percent difference (RPD) equals or exceeds the control criteria. 


\section{A}

\section{Chain of Custody}

4 of 47 


\section{ALS Environmental -- FC \\ Sample Number(s) Cross-Reference Table}

OrderNum: 1209339

Client Name: Battelle Energy Alliance

Client Project Name: MFC Biodiesel Tank Removal ,TOS-A1175 Rev2

Client Project Number: BEA031231

Client PO Number: SOW-8500

\begin{tabular}{|c|c|c|c|c|c|}
\hline $\begin{array}{c}\text { Client Sample } \\
\text { Number }\end{array}$ & $\begin{array}{c}\text { Lab Sample } \\
\text { Number }\end{array}$ & COC Number & Matrix & \begin{tabular}{|c|} 
Date \\
Collected
\end{tabular} & $\begin{array}{c}\text { Time } \\
\text { Collected }\end{array}$ \\
\hline BEA031231_MFC & $1209339-1$ & & SOIL & 20-Sep-12 & $14: 45$ \\
\hline BEA031232_MFC & $1209339-2$ & & SOIL & 20-Sep-12 & $14: 45$ \\
\hline BEA031233_MFC & $1209339-3$ & & SOIL & 20-Sep-12 & $14: 50$ \\
\hline BEA031234_MFC & $1209339-4$ & & SOIL & 20-Sep-12 & $14: 50$ \\
\hline BEA031235_MFC & $1209339-5$ & & SOIL & $20-$ Sep-12 & $14: 55$ \\
\hline BEA031236_MFC & $1209339-6$ & & SOIL & $20-$ Sep-12 & $14: 55$ \\
\hline BEA031237_MFC & $1209339-7$ & & SOIL & $20-$ Sep-12 & 15:00 \\
\hline BEA031238_MFC & $1209339-8$ & & SOIL & 20-Sep-12 & $15: 00$ \\
\hline BEA031239_MFC & $1209339-9$ & & SOIL & $20-$ Sep-12 & $15: 05$ \\
\hline BEA031240_MFC & $1209339-10$ & & SOIL & $20-$ Sep-12 & $15: 05$ \\
\hline BEA031247_MFC & $1209339-11$ & & SOIL & 20-Sep-12 & $15: 10$ \\
\hline BEA031248_MFC & $1209339-12$ & & SOIL & $20-$ Sep-12 & $15: 10$ \\
\hline BEA031249_MFC & $1209339-13$ & & SOIL & 20-Sep-12 & $14: 20$ \\
\hline BEA031250_MFC & $1209339-14$ & & SOIL & 20-Sep-12 & $14: 20$ \\
\hline BEA031251_MFC & $1209339-15$ & & SOIL & 20-Sep-12 & $14: 25$ \\
\hline BEA031252_MFC & $1209339-16$ & & SOIL & $20-\operatorname{Sep}-12$ & $14: 25$ \\
\hline BEA031253_MFC & $1209339-17$ & & SOIL & $20-$ Sep-12 & $14: 30$ \\
\hline BEA031254_MFC & $1209339-18$ & & SOIL & $20-$ Sep-12 & $14: 30$ \\
\hline
\end{tabular}

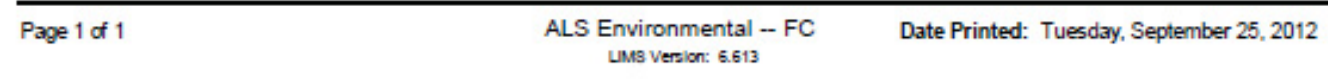




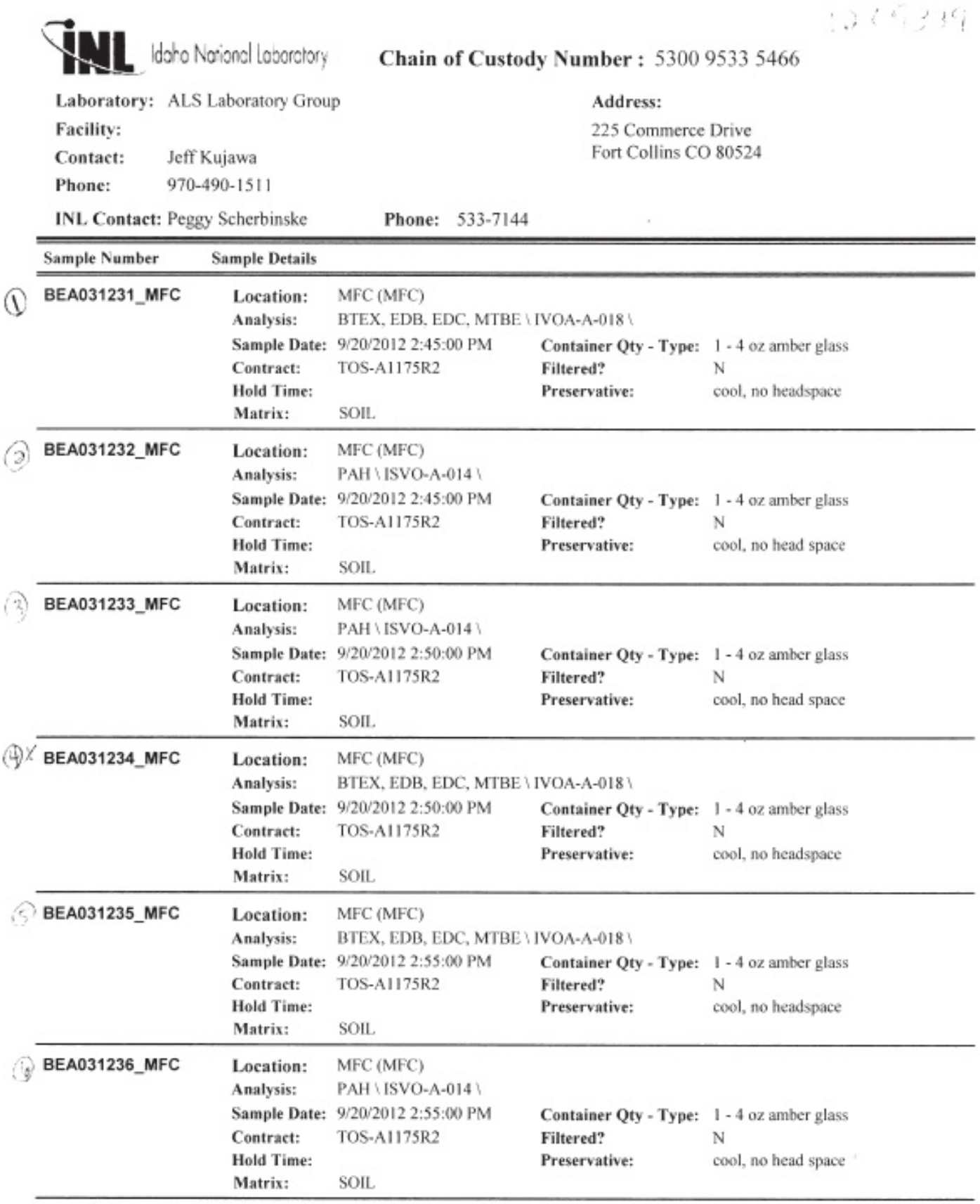




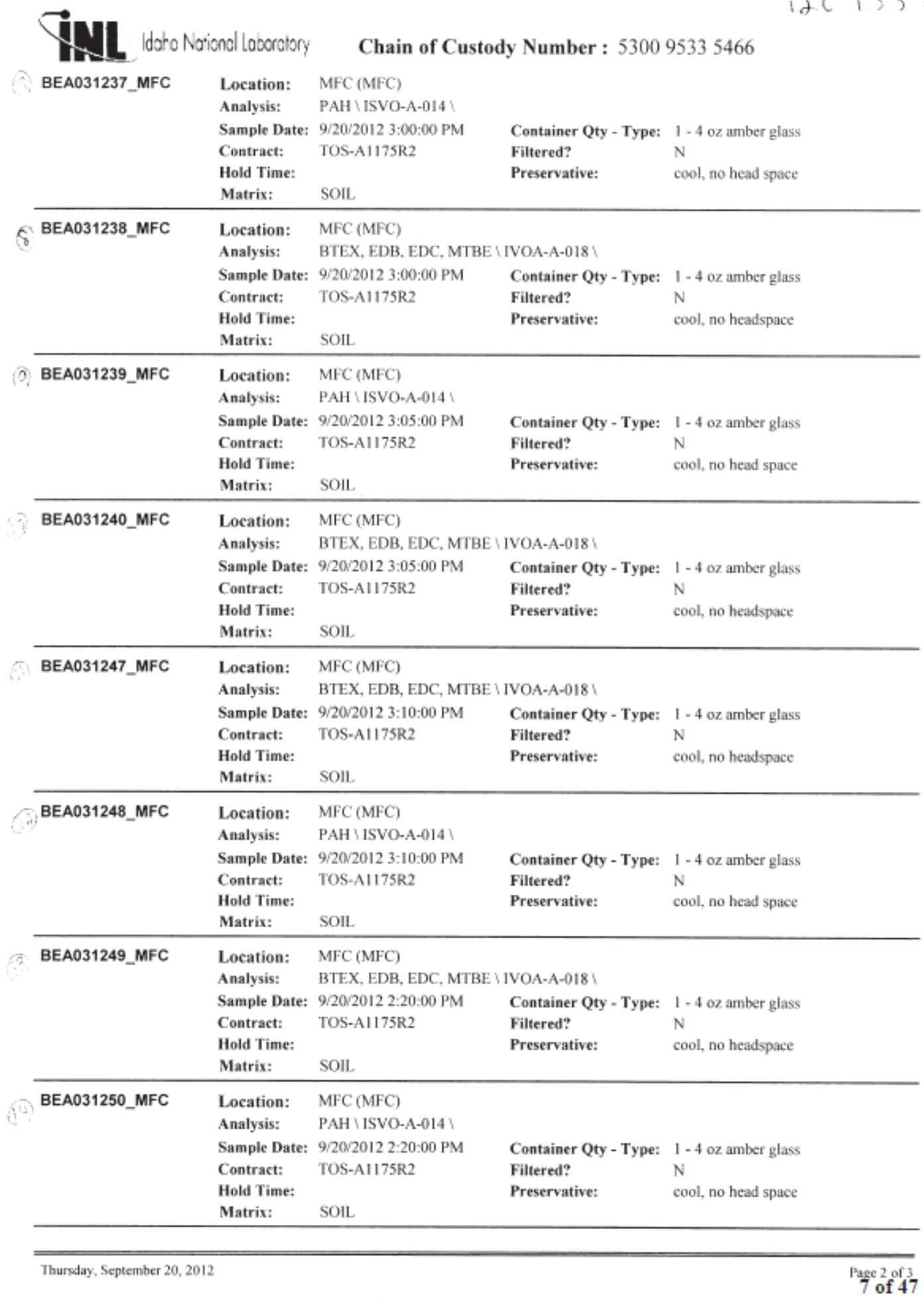




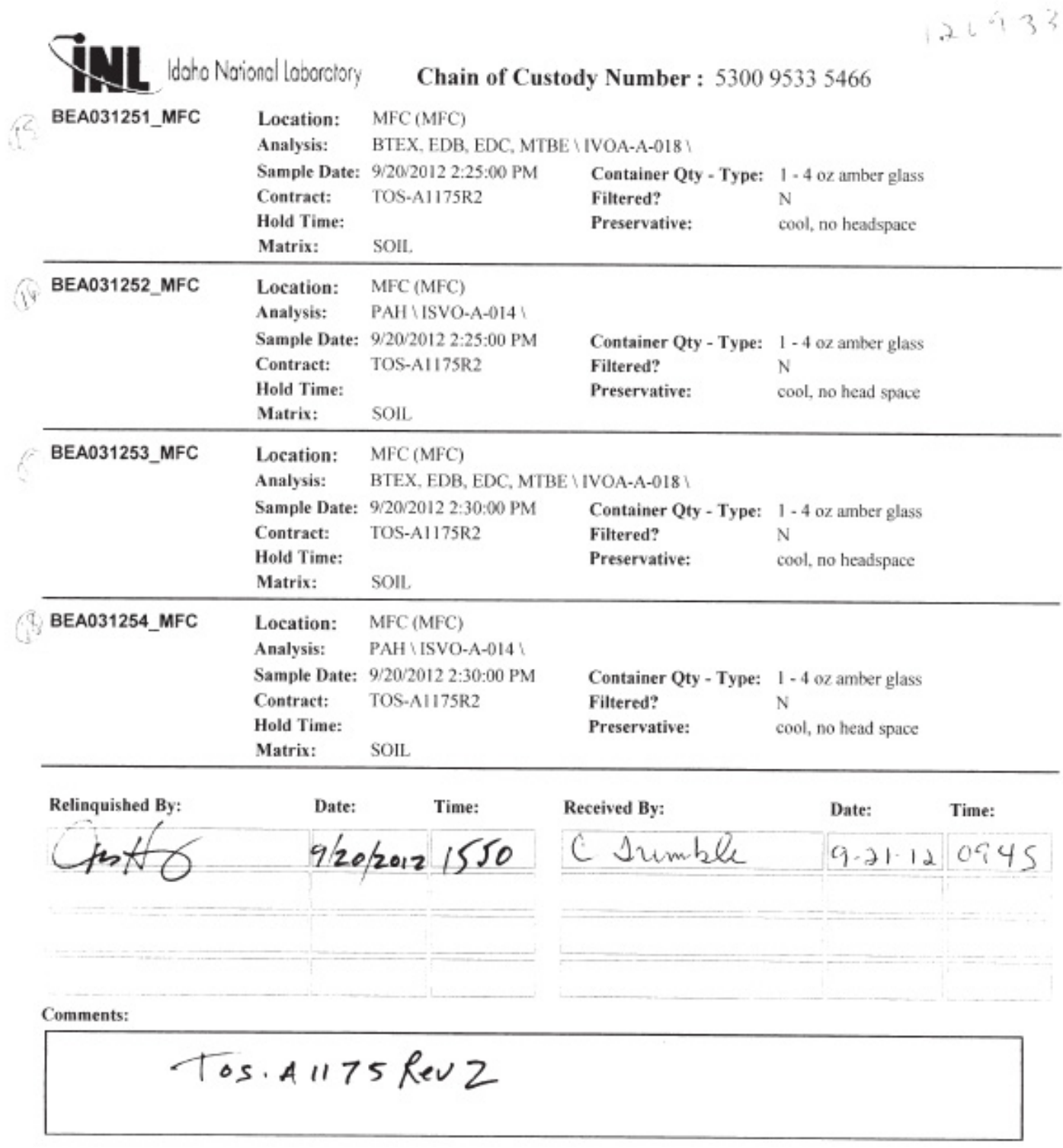




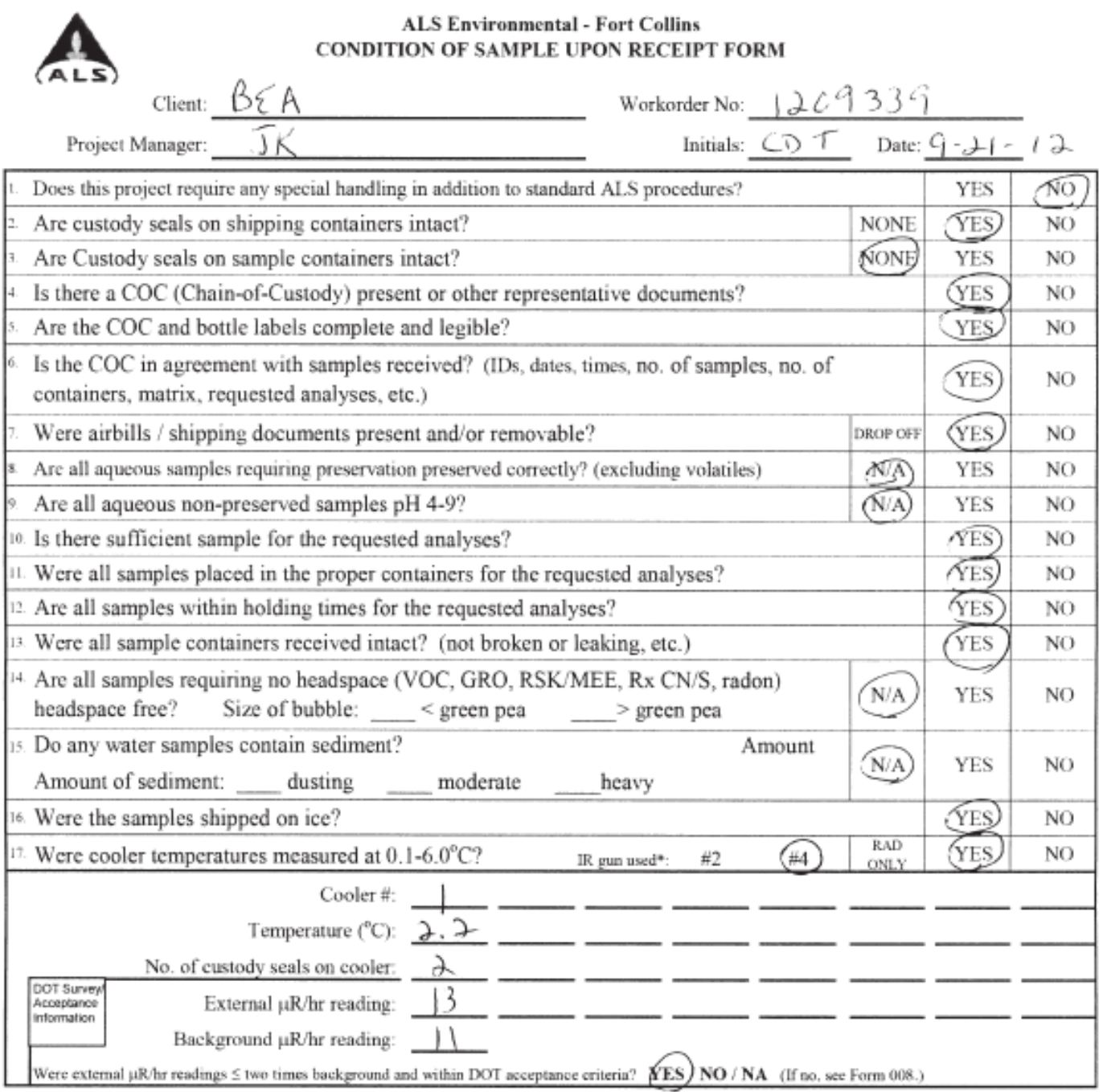

Additional Information: PROVIDE DETALLS BELOW FOR A NO RESPONSE TO ANY QUESTON ABOVE, EXCEPT \#I AND \#16.

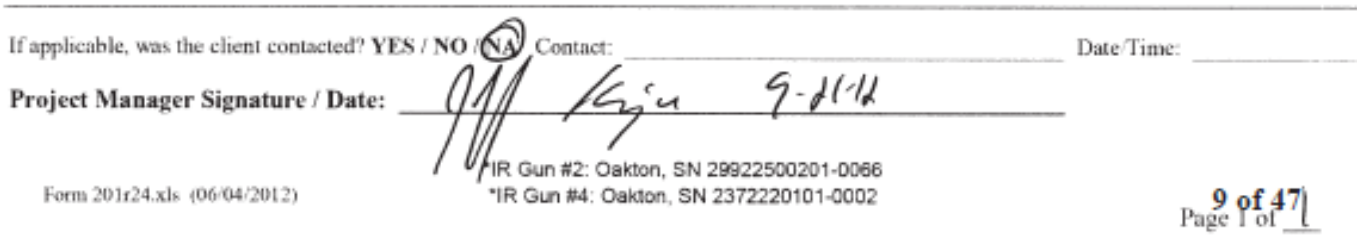




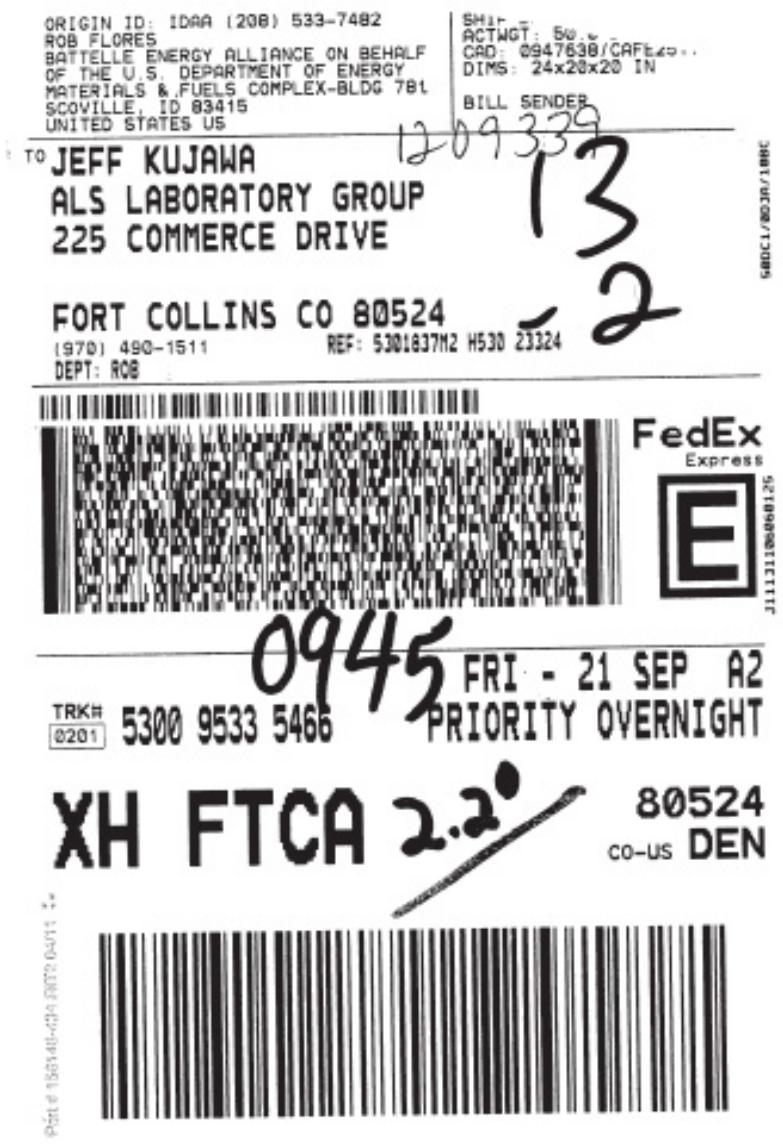

10 of 47 


\section{A}

\section{Analytical Results}




\title{
GC/MS Semi-volatiles
}

\author{
Method SW8270SIMPAHD \\ Method Blank
}

Lab Name: ALS Environmental - FC

Work Order Number: 1209339

Client Name: Battele Energy Alliance

ClientProject ID: MFC Biodiesel Tank Removal ,TOS-A1175 Rev2 BEA031231

\begin{tabular}{|c|c|c|c|c|c|c|}
\hline Lab ID: EX120921 & $\begin{array}{r}\text { Sample Ma } \\
\% \text { Moist } \\
\text { Date Collec } \\
\text { Date Extrac } \\
\text { Date Analyz } \\
\text { Prep Meth }\end{array}$ & $\begin{array}{l}12 \\
12 \\
\text { Rev C }\end{array}$ & $\begin{array}{r}\text { Prep Batch: } \\
\text { QCBatchID: } \\
\text { Run ID: } \\
\text { Cleanup: } \\
\text { Basis: } \\
\text { File Name: }\end{array}$ & $\begin{array}{l}\text { EX120921-6 } \\
\text { EX120921-6-1 } \\
\text { SV120922-1 } \\
\text { NONE } \\
\text { VA } \\
\text { N6033 }\end{array}$ & $\begin{array}{r}\text { Sample } \\
\text { Final } \\
\text { ResL } \\
0\end{array}$ & $\begin{array}{l}\text { iquot: } \\
\text { lume: } \\
\text { Units: UG/KC } \\
\text { an DF: }\end{array}$ \\
\hline CASNO & Target Analyte & DF & Result & $\begin{array}{c}\text { Reporting } \\
\text { Limit }\end{array}$ & $\begin{array}{l}\text { Result } \\
\text { Qualifier }\end{array}$ & $\begin{array}{c}\text { EPA } \\
\text { Qualifier }\end{array}$ \\
\hline $91-20-3$ & NAPHTHALENE & 1 & 3.3 & 3.3 & $u$ & \\
\hline $208-96-8$ & ACENAPHTHYLENE & 1 & 3.3 & 3.3 & U & \\
\hline $83-32-8$ & ACENAPHTHENE & 1 & 3.3 & 3.3 & $u$ & \\
\hline $86-73-7$ & FLUORENE & 1 & 3.3 & 3.3 & u & \\
\hline $85-01-8$ & PHENANTHRENE & 1 & 3.3 & 3.3 & $u$ & \\
\hline $120-12-7$ & ANTHRACENE & 1 & 3.3 & 3.3 & $u$ & \\
\hline $206-44-0$ & FLUORANTHENE & 1 & 3.3 & 3.3 & $u$ & \\
\hline $129-00-0$ & PYRENE & 1 & 3.3 & 3.3 & $u$ & \\
\hline $56-55-3$ & BENZO(A)ANTHRACENE & 1 & 3.3 & 3.3 & $u$ & \\
\hline $218-01-9$ & CHRYSENE & 1 & 3.3 & 3.3 & $u$ & \\
\hline $205-80-2$ & BENZO(B)FLUORANTHENE & 1 & 3.3 & 3.3 & $u$ & \\
\hline $207-08-9$ & BENZO(K)FLUORANTHENE & 1 & 3.3 & 3.3 & $u$ & \\
\hline $50-32-8$ & BENZO(A)PYRENE & 1 & 3.3 & 3.3 & $u$ & \\
\hline $193-39-5$ & INDENO(1,2,3-CD)PYRENE & 1 & 3.3 & 3.3 & $u$ & \\
\hline $53-70-3$ & DBBENZO(A,H)ANTHRACENE & 1 & 3.3 & 3.3 & $u$ & \\
\hline $191-24-2$ & BENZO(G,H,J)PERYLENE & 1 & 3.3 & 3.3 & $u$ & \\
\hline
\end{tabular}

Surrogate Recovery

\begin{tabular}{|l|l|r|r|r|r|c|}
\hline CASNO & \multicolumn{1}{|c|}{ Surrogate Analyte } & Result & Flag & $\begin{array}{c}\text { Spike } \\
\text { Amount }\end{array}$ & $\begin{array}{c}\text { Percent } \\
\text { Recovery }\end{array}$ & $\begin{array}{c}\text { Control } \\
\text { Limits }\end{array}$ \\
\hline $321-60-8$ & 2-FLUOROBIPHENYL & 48.4 & & 68.7 & 73 & $41-106$ \\
\hline $4165-60-0$ & NTRROBENZENE-DS & 53 & & 68.7 & 80 & $28-113$ \\
\hline $1718-51-0$ & TERPHENYL-D14 & 45 & & 68.7 & 68 & $25-147$ \\
\hline
\end{tabular}

Data Package ID: SV1209339-1

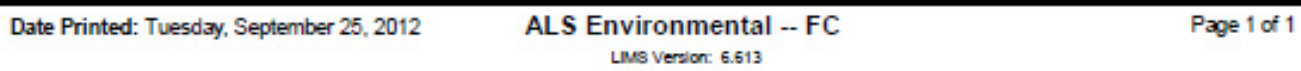

12 of 47 


\section{GUIIIO OCIIIIVUIatico}

\section{Method SW8270SIMPAH Revision D}

Sample Results

Lab Name: ALS Environmental - FC

Work Order Number: 1209339

Client Name: Battele Energy Alliance

ClientProject ID: MFC Biodiesel Tank Removal ,TOS-A1175 ReV2 BEA031231

\begin{tabular}{|c|c|c|c|c|c|c|}
\hline \multirow[t]{3}{*}{$\begin{aligned} & \text { Field ID: } \text { BEAD31. } \\
& \text { Lab ID: } 1209339 \\
&\end{aligned}$} & $\begin{array}{l}\text { Sample } \\
\% \text { Mo } \\
\text { Date Col }\end{array}$ & \multirow{3}{*}{$\begin{array}{l}\text { Sample Matrix: SOIL } \\
\quad \% \text { Moisture: } 9.2 \\
\text { Date Collected: } 20-\text { Sep-12 } \\
\text { Date Extracted: } 21-\text { Sep-12 } \\
\text { Date Analyzed: } 22-\text { Sep-12 } \\
\text { Prep Method: SW3540 Rev C }\end{array}$} & \multirow{3}{*}{\multicolumn{2}{|c|}{$\begin{array}{l}\text { Prep Batch: EX120921-6 } \\
\text { QCBatchID: EX120921-6-1 } \\
\text { Run ID: SV120922-1 } \\
\text { Cleanup: NONE } \\
\text { Basis: DryWeight } \\
\text { File Name: N6049 }\end{array}$}} & \multicolumn{2}{|c|}{$\begin{array}{cr}\text { Sample Aliquot: } & 30.31 \mathrm{G} \\
\text { Final Volume: } & 1 \mathrm{ML}\end{array}$} \\
\hline & Date Ext & & & & \multicolumn{2}{|c|}{ Result Units: UGIKG } \\
\hline & Date Anz & & & & & $\mathrm{F}: \quad 1$ \\
\hline CASNO & Target Analyte & $\begin{array}{c}\text { Dilution } \\
\text { Factor }\end{array}$ & Result & $\begin{array}{l}\text { Reporting } \\
\text { Limit }\end{array}$ & $\begin{array}{l}\text { Result } \\
\text { Qualifier }\end{array}$ & $\begin{array}{c}\text { EPA } \\
\text { Qualifier }\end{array}$ \\
\hline $91-20-3$ & NAPHTHALENE & 1 & 3.6 & 3.6 & $u$ & \\
\hline $208-86-8$ & ACENAPHTHYLENE & 1 & 3.6 & 3.6 & $u$ & \\
\hline $83-32-9$ & ACENAPHTHENE & 1 & 3.6 & 3.6 & $u$ & \\
\hline $86-73-7$ & FLUORENE & 1 & 3.6 & 3.6 & $u$ & \\
\hline $85-01-8$ & PHENANTHRENE & 1 & 9.6 & 3.6 & & \\
\hline $120-12-7$ & ANTHRACENE & 1 & 3.2 & 3.6 & $\mathrm{~J}$ & \\
\hline $206-44-0$ & FLUORANTHENE & 1 & 68 & 3.6 & & \\
\hline $129-00-0$ & PYRENE & 1 & 61 & 3.6 & & \\
\hline $56-55-3$ & BENZO(A)ANTHRACENE & 1 & 44 & 3.6 & & \\
\hline $218-01-9$ & CHRYSENE & 1 & 42 & 3.6 & & \\
\hline $205-89-2$ & BENZO;B]FLUORANTHENE & 1 & 99 & 3.6 & & \\
\hline $207-08-9$ & BENZO|K|FLUORANTHENE & 1 & 40 & 3.6 & & \\
\hline $50-32-8$ & BENZOYA]PYRENE & 1 & 60 & 3.6 & & \\
\hline $193-39-5$ & INDENO $(1,2,3-C D)$ PYRENE & 1 & 25 & 3.6 & & \\
\hline $53-70-3$ & DIBENZO(A,H)ANTHRACENE & 1 & 9.3 & 3.6 & & \\
\hline $191-24-2$ & BENZO,G,H,DPERYLENE & 1 & 30 & 3.6 & & \\
\hline
\end{tabular}

\section{Surrogate Recovery}

\begin{tabular}{|l|l|r|r|r|r|r|}
\hline CASNO & \multicolumn{1}{|c|}{ Surrogate Analyte } & Result & Flag & $\begin{array}{c}\text { Spike } \\
\text { Amount }\end{array}$ & $\begin{array}{c}\text { Percent } \\
\text { Recovery }\end{array}$ & $\begin{array}{c}\text { Control } \\
\text { Limits }\end{array}$ \\
\hline $321-60-8$ & 2-FLUOROBIPHENYL & 55.1 & & 72.6 & 76 & $41-106$ \\
\hline $4165-60-0$ & NIT ROEENZENE-D5 & 60.3 & & 72.6 & 83 & $28-113$ \\
\hline $1718-51-0$ & TERPHENYL-D14 & 68.7 & & 72.6 & 85 & $25-147$ \\
\hline
\end{tabular}

Data Package ID: SV1209339-1

$\begin{array}{cc}\text { Date Printed: Tuesday, September 25, } 2012 & \text { ALS Environmental -- FC } \\ \text { LMS version: } 6.613 & \text { Page } 1 \text { of } 10\end{array}$

13 of 47 


\section{GC/MS Semi-volatiles}

\section{Method SW8270SIMPAH Revision D \\ Sample Results}

Lab Name: ALS Environmental - FC

Work Order Number: 1208339

Client Name: Battele Energy Alliance

ClientProject ID: MFC Biodiesel Tank Removal ,TOS-A1175 ReV2 BEA031231

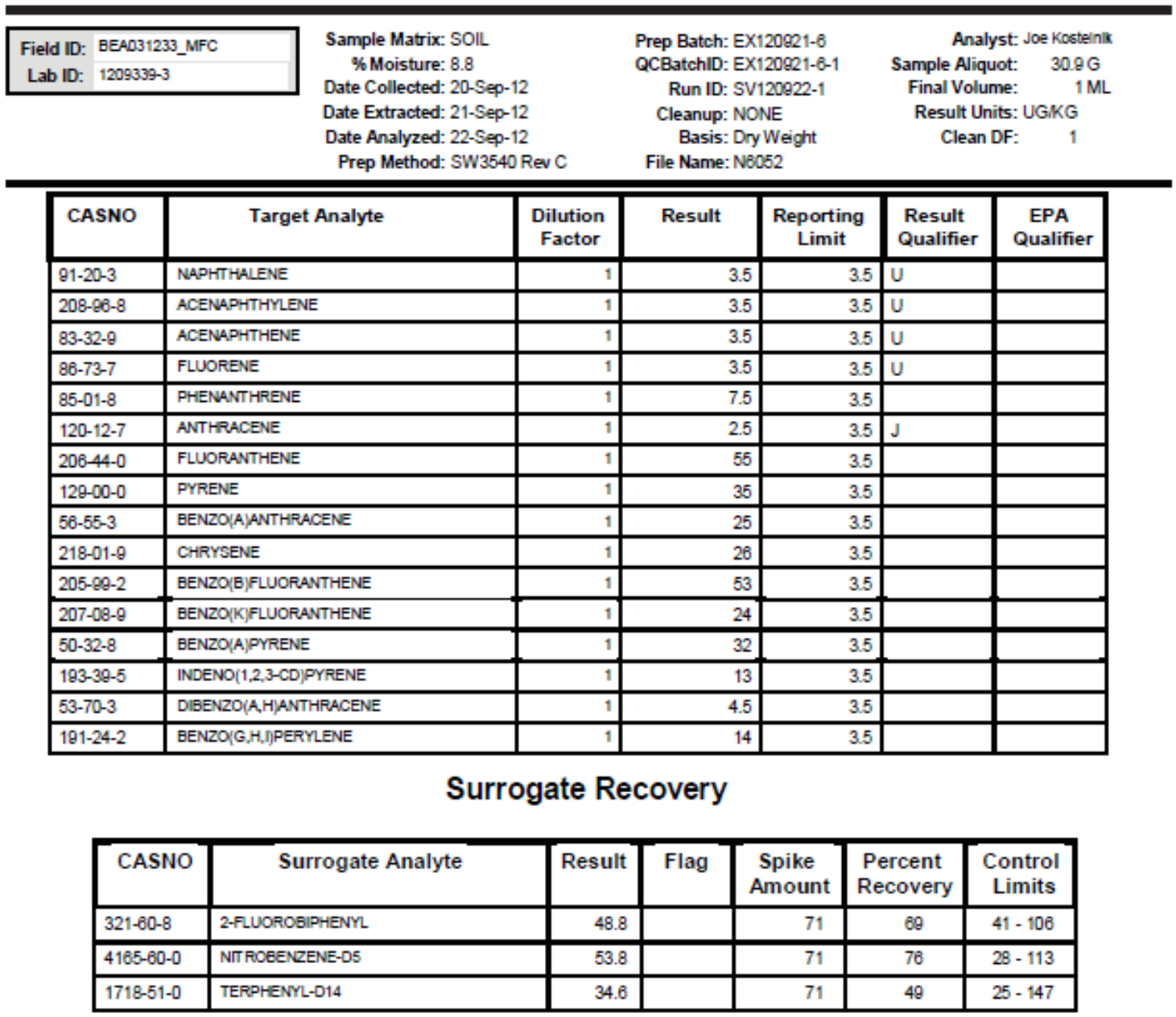

Data Package ID: SV1209339-1

$\begin{array}{cc}\text { Date Printed: Tuesday, September 25, } 2012 & \text { ALS Environmental -- FC } \\ \text { LIMS Version: } 6.613 & \text { Page } 2 \text { of } 10\end{array}$

14 of 47 


\section{GC/MS Semi-volatiles}

\section{Method SW8270SIMPAH Revision D \\ Sample Results}

Lab Name: ALS Environmental - FC

Work Order Number: 1208339

Client Name: Battele Energy Alliance

ClientProject ID: MFC Biodiesel Tank Remova ,TOS-A1175 Rev2 BEA031231

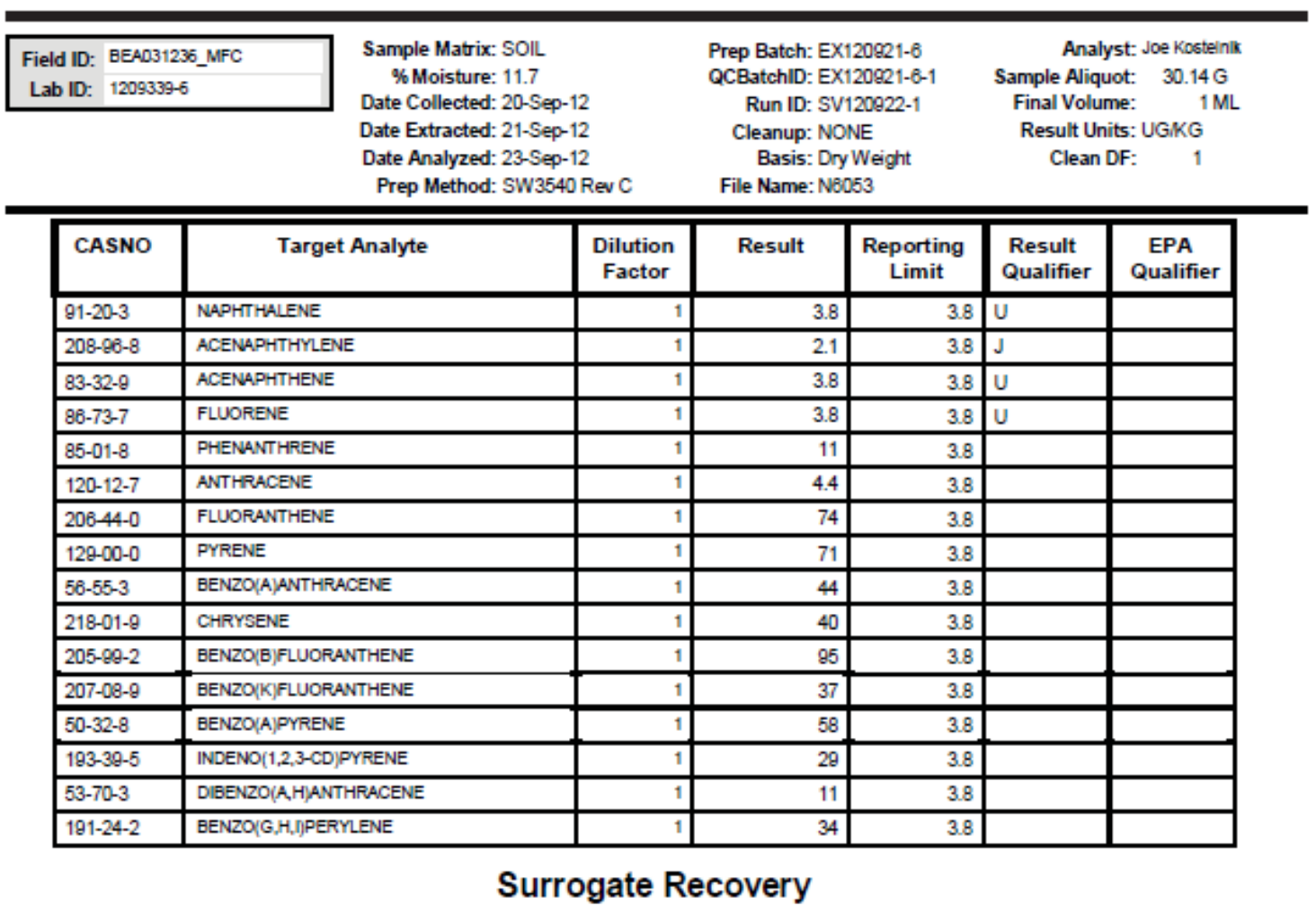

\begin{tabular}{|l|l|r|r|r|r|r|}
\hline CASNO & \multicolumn{1}{|c|}{ Surrogate Analyte } & Result & Flag & $\begin{array}{c}\text { Spike } \\
\text { Amount }\end{array}$ & $\begin{array}{c}\text { Percent } \\
\text { Recovery }\end{array}$ & $\begin{array}{c}\text { Control } \\
\text { Limits }\end{array}$ \\
\hline $321-60-8$ & 2-FLUOROBIPHENYL & 52.6 & & 75.1 & 70 & $41-106$ \\
\hline $4165-60-0$ & NIT ROEENZENE-D5 & 59.6 & & 75.1 & 79 & $28-113$ \\
\hline $1718-51-0$ & TERPHENYL-D14 & 80.5 & & 75.1 & 107 & $25-147$ \\
\hline
\end{tabular}

Data Package ID: SV1209339-1

Date Printed: Tuesday, September 25, 2012

ALS Environmental -- FC

LMS Version: 6.613
Page 3 of 10

15 of 47 


\title{
GC/MS Semi-volatiles
}

\author{
Method SW8270SIMPAH Revision D
}

Sample Results

Lab Name: ALS Environmental - FC

Work Order Number: 1209339

Client Name: Battele Energy Alliance

ClientProject ID: MFC Biodiesel Tank Removal,TOS-A1175 Rev2 BEA031231

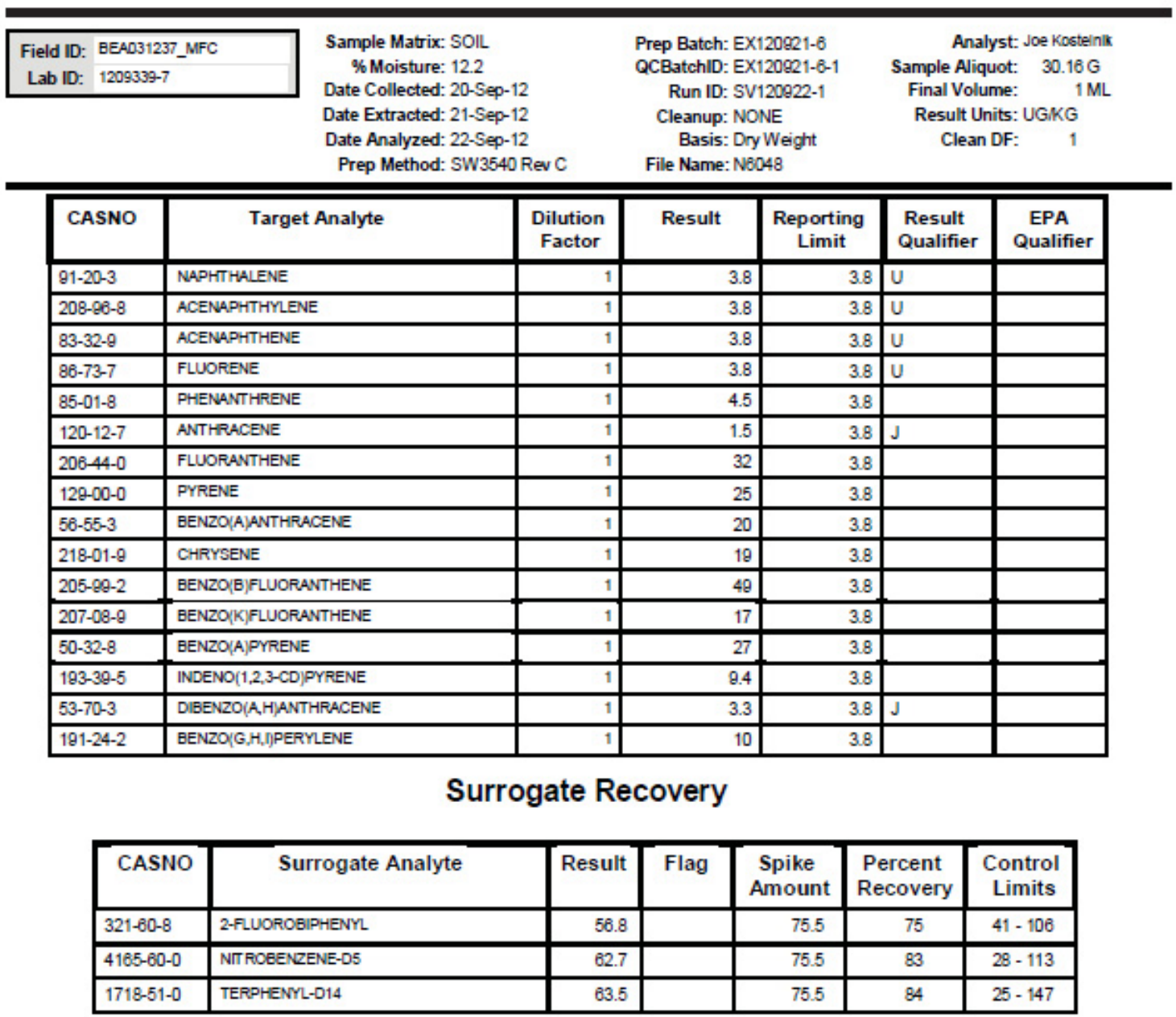

Data Package ID: SV1209339-1

Date Printed: Tuesday, September 25, 2012
ALS Environmental -. FC

LIMS Version: 6.613
Page 4 of 10

16 of 47 


\section{GC/MS Semi-volatiles}

\section{Method SW8270SIMPAH Revision D \\ Sample Results}

Lab Name: ALS Environmental - FC

Work Order Number: 1208339

Client Name: Battele Energy Alliance

ClientProject ID: MFC Biodiesel Tank Removal ,TOS-A1175 ReV2 BEA031231

\begin{tabular}{|c|c|c|c|}
\hline \multirow{5}{*}{$\begin{aligned} \text { Field ID: } & \text { BEAD31239_MFC } \\
\text { Lab ID: } & 1209339-9\end{aligned}$} & Sample Matrix: SOIL & Prep Batch: EX120921-6 & Analyst: Joe Kostein' \\
\hline & $\begin{array}{l}\text { \% Moisture: } 11.9 \\
\text { Date Collected: } 20-\text { Sep-12 }\end{array}$ & $\begin{array}{l}\text { QCBatchID: EX120921-6-1 } \\
\text { Run ID: SV120922-1 }\end{array}$ & $\begin{array}{l}\text { Sample Aliquot: } \\
\text { Final Volume: }\end{array}$ \\
\hline & Date Extracted: $21-$ Sep-12 & Cleanup: NONE & Result Units: UGIKG \\
\hline & Date Analyzed: 23-Sep-12 & Basis: Dry Weight & Clean DF: \\
\hline & Prep Method: SW3540 Rev C & File Name: N6054 & \\
\hline
\end{tabular}

\begin{tabular}{|c|c|c|c|c|c|c|}
\hline CASNO & Target Analyte & $\begin{array}{c}\text { Dilution } \\
\text { Factor }\end{array}$ & Result & $\begin{array}{c}\text { Reporting } \\
\text { Limit }\end{array}$ & $\begin{array}{l}\text { Result } \\
\text { Qualifier }\end{array}$ & $\begin{array}{c}\text { EPA } \\
\text { Qualifier }\end{array}$ \\
\hline $91-20-3$ & NAPHTHALENE & 1 & 3.8 & 3.8 & $u$ & \\
\hline $208-86-8$ & ACENAPHTHYLENE & 1 & 3.8 & 3.8 & $U$ & \\
\hline $83-32-9$ & ACENAPHTHENE & 1 & 3.8 & 3.8 & U & \\
\hline $86-73-7$ & FLUORENE & 1 & 3.8 & 3.8 & $u$ & \\
\hline $85-01-8$ & PHENANTHRENE & 1 & 5.5 & 3.8 & & \\
\hline $120-12-7$ & ANTHRACENE & 1 & 1.8 & 3.8 & $\mathrm{~J}$ & \\
\hline $206-44-0$ & FLUORANTHENE & 1 & 45 & 3.8 & & \\
\hline $129-00-0$ & PYRENE & 1 & 56 & 3.8 & & \\
\hline $56-55-3$ & BENZOYA]ANTHRACENE & 1 & 35 & 3.8 & & \\
\hline $218-01-9$ & CHRYSENE & 1 & 36 & 3.8 & & \\
\hline $205-8 \theta-2$ & BENZO(B)FLUORANTHENE & 1 & 72 & 3.8 & & \\
\hline $207-08-9$ & BENZO|K|FLUORANTHENE & 1 & 31 & 3.8 & & \\
\hline $50-32-8$ & BENZO[A]PYRENE & 1 & 41 & 3.8 & & \\
\hline $193-39-5$ & INDENO(1,2,3-CD)PYRENE & 1 & 18 & 3.8 & & \\
\hline $53-70-3$ & DIBENZO(A,H)ANTHRACENE & 1 & 6.4 & 3.8 & & \\
\hline $191-24-2$ & BENZO,G,H,J)PERYLENE & 1 & 23 & 3.8 & & \\
\hline
\end{tabular}

Surrogate Recovery

\begin{tabular}{|l|l|r|r|r|r|r|}
\hline CASNO & \multicolumn{1}{|c|}{ Surrogate Analyte } & Result & Flag & $\begin{array}{c}\text { Spike } \\
\text { Amount }\end{array}$ & $\begin{array}{c}\text { Percent } \\
\text { Recovery }\end{array}$ & $\begin{array}{c}\text { Control } \\
\text { Limits }\end{array}$ \\
\hline $321-60-8$ & 2-FLUOROBIPHENYL & 49.1 & & 75.2 & 65 & $41-106$ \\
\hline $4185-60-0$ & NTR ROEENZENE-D5 & 49.9 & & 75.2 & 68 & $28-113$ \\
\hline $1718-51-0$ & TERPHENYL-D14 & 80.6 & & 75.2 & 107 & $25-147$ \\
\hline
\end{tabular}

Data Package ID: SV1209339-1

\begin{tabular}{|c|c|}
\hline Date Printed: Tuesday, September 25, 2012 & $\begin{array}{c}\text { ALS Environmental -- FC } \\
\text { LMS Version: } 6.613\end{array}$ \\
\hline
\end{tabular}

17 of 47 


\section{GC/MS Semi-volatiles}

\section{Method SW8270SIMPAH Revision D \\ Sample Results}

Lab Name: ALS Environmental - FC

Work Order Number: 1208339

Client Name: Battele Energy Alliance

ClientProject ID: MFC Biodiesel Tank Remova ,TOS-A1175 Rev2 BEA031231

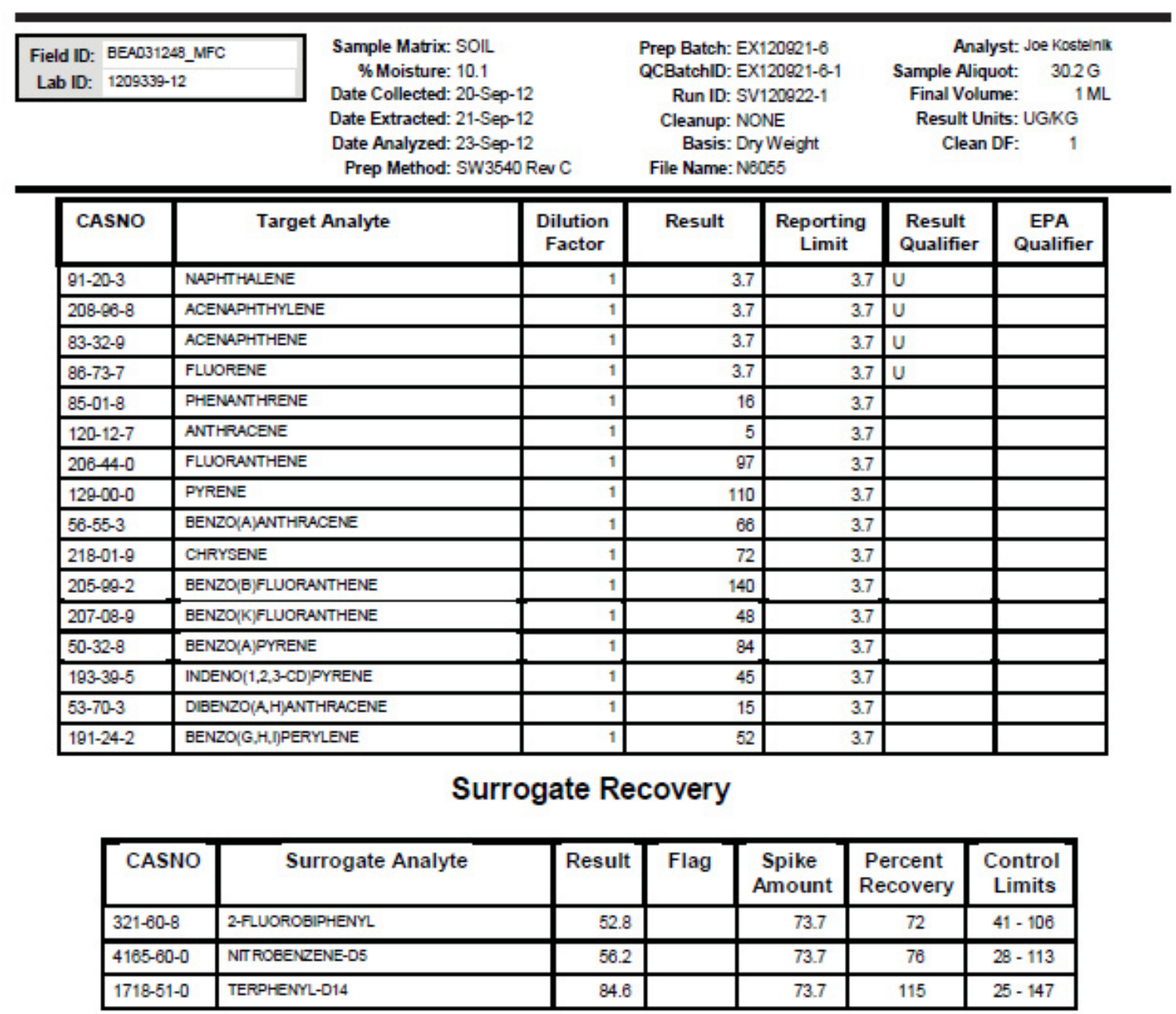

Data Package ID: SV1209339-1

$\begin{array}{ccc}\text { Date Printed: Tuesday, September 25, 2012 } & \text { ALS Environmental -- FC } \\ \text { LMS Version: } 6.613 & \text { Page } 6 \text { of } 10\end{array}$

18 of 47 


\section{GC/MS Semi-volatiles}

\section{Method SW8270SIMPAH Revision D \\ Sample Results}

Lab Name: ALS Environmental - FC

Work Order Number: 1208339

Client Name: Battele Energy Alliance

ClientProject ID: MFC Biodiesel Tank Removal ,TOS-A1175 Rev2 BEA031231

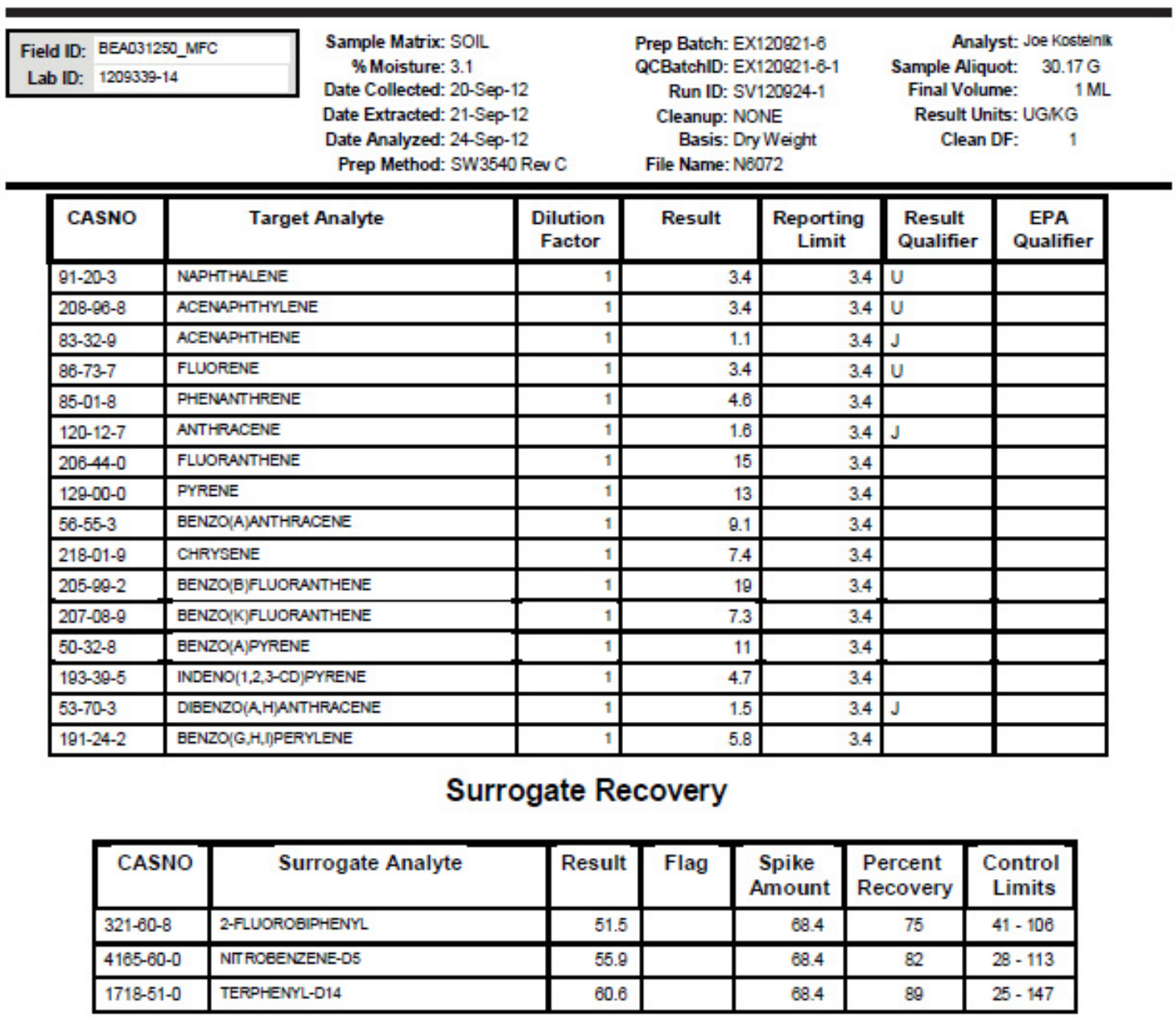

Data Package ID: SV1209339-1

$\begin{array}{lc}\text { Date Printed: Tuesday, September 25, } 2012 & \text { ALS Environmental -- FC } \\ \text { LMS Version: } 6.613 & \text { Page } 7 \text { of } 10\end{array}$

19 of 47 


\section{GC/MS Semi-volatiles}

\section{Method SW8270SIMPAH Revision D \\ Sample Results}

Lab Name: ALS Environmental - FC

Work Order Number: 1208339

Client Name: Battele Energy Alliance

ClientProject ID: MFC Biodiesel Tank Removal ,TOS-A1175 ReV2 BEA031231

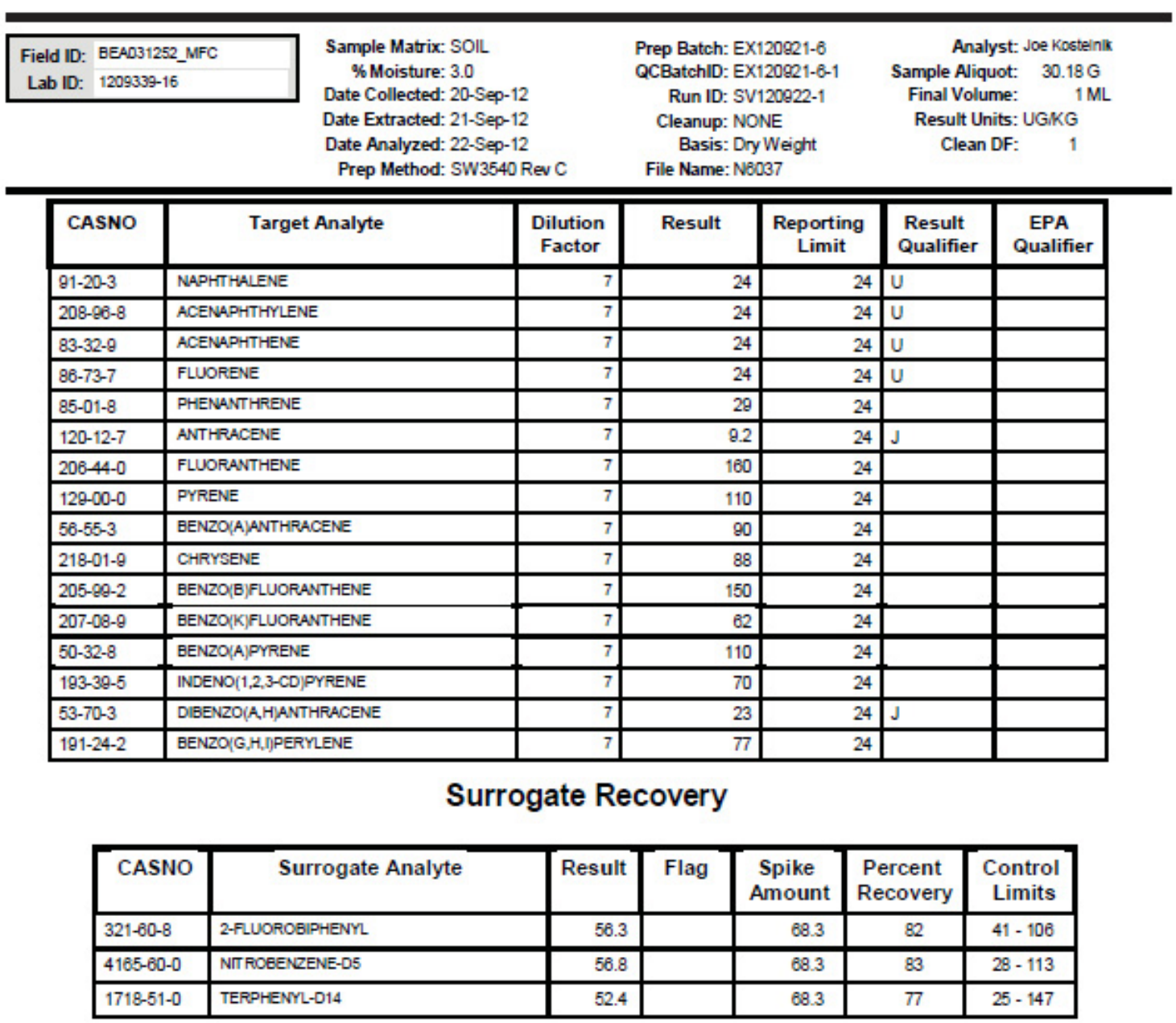

Data Package ID: SV1209339-1

Date Printed: Tuesday, September 25, $2012 \quad$ ALS Environmental -- FC
LMS Version: 6.613

20 of 47 


\section{GC/MS Semi-volatiles}

\section{Method SW8270SIMPAH Revision D \\ Sample Results}

Lab Name: ALS Environmental - FC

Work Order Number: 1209339

Client Name: Battele Energy Alliance

ClientProject ID: MFC Biodiesel Tank Removal ,TOS-A1175 ReV2 BEA031231

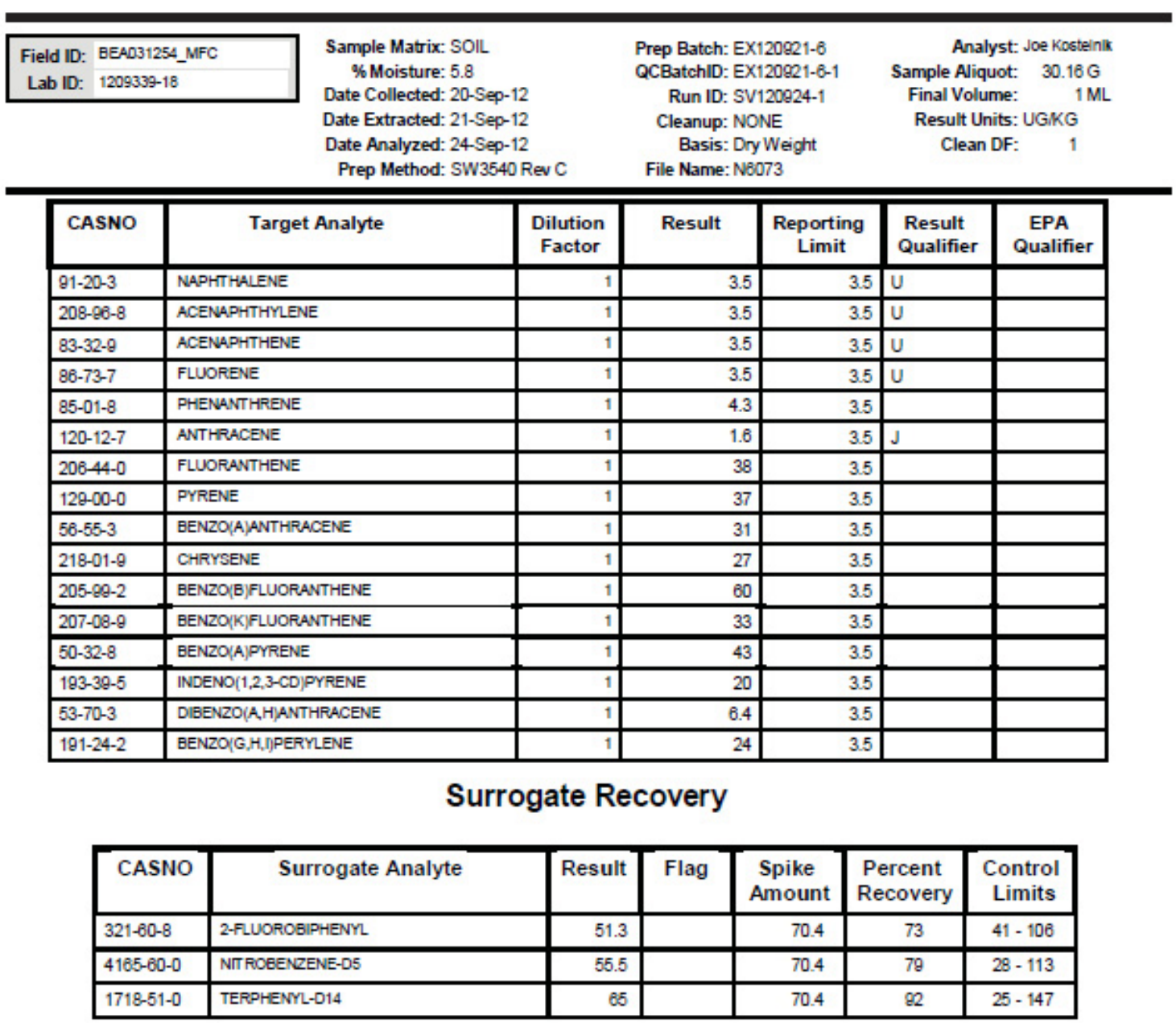

Data Package ID: SV1209339-1

Date Printed: Tuesday, September 25, 2012 ALS Environmental -- FC
LMS Version: 6.613

21 of 47 


\section{GC/MS Semi-volatiles}

\section{Method SW8270SIMPAH Revision D \\ Sample Results}

Lab Name: ALS Environmental - FC

Work Order Number: 1209339

Client Name: Battele Energy Alliance

ClientProject ID: MFC Biodiesel Tank Remova ,TOS-A1175 Rev2 BEA031231

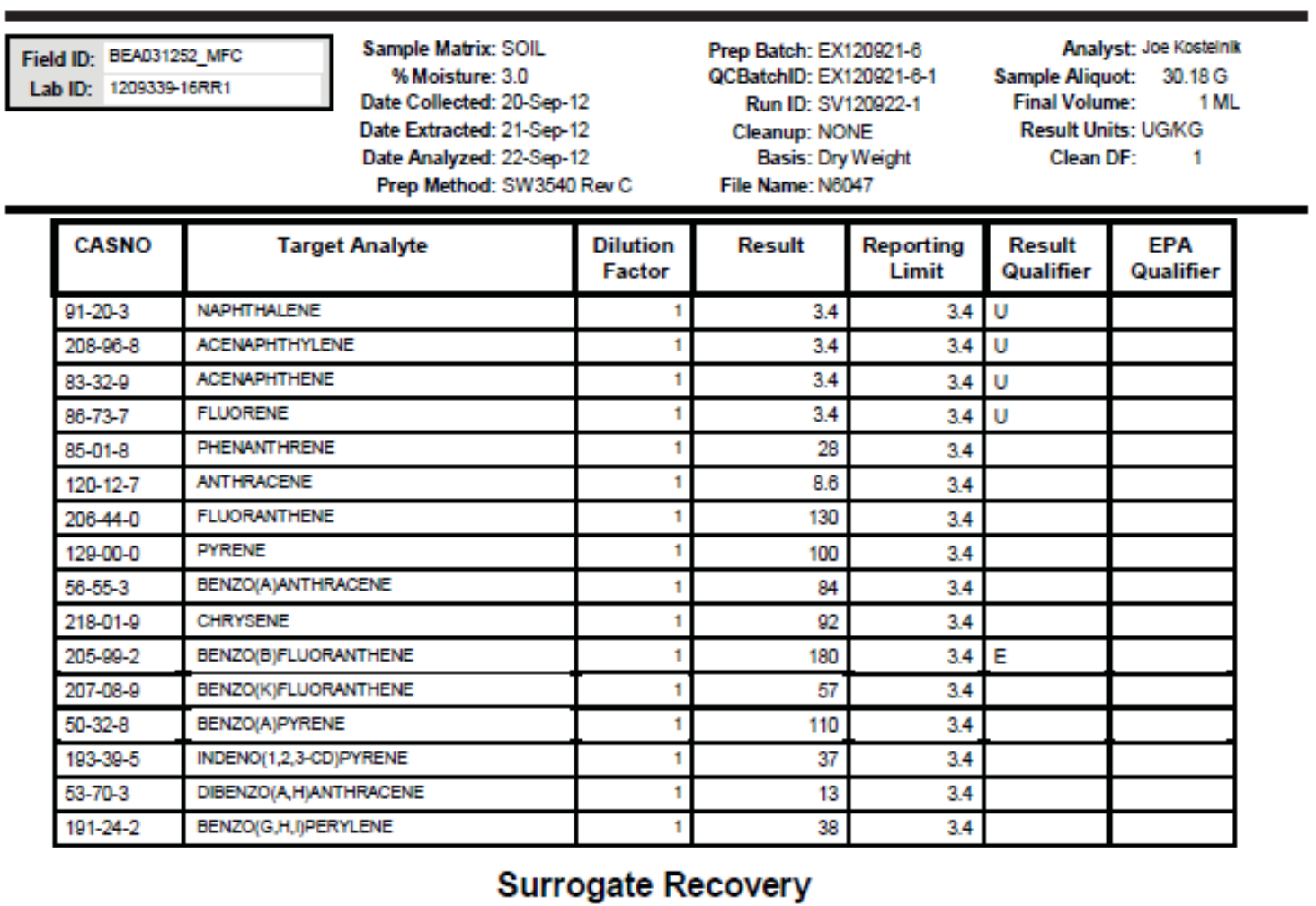

\begin{tabular}{|l|l|r|r|r|r|c|}
\hline CASNO & \multicolumn{1}{|c|}{ Surrogate Analyte } & Result & Flag & $\begin{array}{c}\text { Spike } \\
\text { Amount }\end{array}$ & $\begin{array}{c}\text { Percent } \\
\text { Recovery }\end{array}$ & $\begin{array}{c}\text { Control } \\
\text { Limits }\end{array}$ \\
\hline $321-60-8$ & 2-FLUOROBIPHENYL & 48.9 & & 68.3 & 72 & $41-106$ \\
\hline $4185-60-0$ & NTROEENZENE-D5 & 52.8 & & 68.3 & 77 & $28-113$ \\
\hline $1718-51-0$ & TERPHENYL-D14 & 54.6 & & 68.3 & 80 & $25-147$ \\
\hline
\end{tabular}

Data Package ID: SV1209339-1

Date Printed: Tuesday, September 25, 2012

ALS Environmental -- FC

LMS Version: 6.613

Page 10 of 10

22 of 47 


\section{$A$}

\section{Supporting QA/QC Data}




\title{
Surrogate Summary for GC/MS Semi-volatiles
}

\author{
Method SW8270SIMPAHD
}

Lab Name: ALS Environmental - FC

Work Order Number: 1209339

Client Name: Battelle Energy Alliance

ClientProject ID: MFC Biodiesel Tank Remova ,TOS-A1175 Rev2 BEA031231

\begin{tabular}{|c|c|c|c|}
\hline \multirow{2}{*}{$\begin{array}{l}\text { PrepBatchID: EX120921-6 } \\
\text { QC Batch ID: EX120921-6-1 }\end{array}$} & Surrogate Compound & \multicolumn{2}{|c|}{ Control Limits } \\
\hline & 2,4,6-Tribromophenol & & \\
\hline \multirow[t]{5}{*}{ Date Extracted: 9/21/2012 } & 2-Fluorobiphenyl & 41 & 106 \\
\hline & 2-Fluorophend & & \\
\hline & Nitrobenzene-d5 & 28 & 113 \\
\hline & Phenol-d5 & & \\
\hline & Terphenyi-d14 & 25 & 147 \\
\hline
\end{tabular}

\begin{tabular}{|c|c|c|c|c|c|c|c|c|c|}
\hline Lab ID & Client Sample ID & $\begin{array}{c}\text { Date } \\
\text { Collected }\end{array}$ & $\begin{array}{c}\text { Date } \\
\text { Received }\end{array}$ & $\begin{array}{l}246 T B \% \\
\text { Recovery }\end{array}$ & $\begin{array}{l}\text { 2FBP \% } \\
\text { Recovery }\end{array}$ & $\begin{array}{c}\text { 2FP \% } \\
\text { Recovery }\end{array}$ & $\begin{array}{c}\text { ND5\% } \% \\
\text { Recovery }\end{array}$ & $\begin{array}{c}\text { PD5 \% } \\
\text { Recovery }\end{array}$ & $\begin{array}{l}\text { TD14\% } \% \\
\text { Recovery }\end{array}$ \\
\hline $1209339-6$ & BEA031236_MFC & $9 / 20 / 2012$ & $9 / 21 / 2012$ & & 70 & & 79 & & 107 \\
\hline $1209339-9$ & BEA031239_MFC & $9 / 20 / 2012$ & $9 / 21 / 2012$ & & 65 & & 66 & & 107 \\
\hline $1209339-12$ & BEA031248_MFC & $9 / 20 / 2012$ & $9 / 21 / 2012$ & & 72 & & 76 & & 115 \\
\hline EX120921-6MB & $x 000 X X 00 X$ & NA & $X 000000$ & & 73 & & 80 & & 68 \\
\hline$\overline{E X 120921-6 L C S}$ & 00000000 & NA & $\mathrm{X000000X}$ & & 78 & & 85 & & 75 \\
\hline EX120921-6LCSD & 10000000 & NA & $x 000000$ & & 72 & & 80 & & 74 \\
\hline $1209339-16$ & BEA031252_MFC & $9 / 20 / 2012$ & $9 / 21 / 2012$ & & 82 & & 83 & & 77 \\
\hline 1209339-16RR1 & BEA031252_MFC & $9 / 20 / 2012$ & $9 / 21 / 2012$ & & 72 & & 77 & & 80 \\
\hline $1209339-7$ & BEA031237_MFC & $9 / 20 / 2012$ & $9 / 21 / 2012$ & & 75 & & 83 & & 84 \\
\hline $1209339-2$ & BEA031232_MFC & $9 / 20 / 2012$ & $9 / 21 / 2012$ & & 78 & & 83 & & 25 \\
\hline 1209339-2MS & BEA031232_MFC & $9 / 20 / 2012$ & $9 / 21 / 2012$ & & 68 & & 71 & & 80 \\
\hline 1209339-2MSD & BEA031232_MFC & $9 / 20 / 2012$ & $9 / 21 / 2012$ & & 71 & & 76 & & 94 \\
\hline $1209339-3$ & BEA031233_MFC & $9 / 20 / 2012$ & $9 / 21 / 2012$ & & 69 & & 76 & & 49 \\
\hline
\end{tabular}

Data Package ID: SV1209339-1

\begin{tabular}{ccc}
\hline Date Printed: Tuesday, September 25,2012 & ALS Environmental - FC \\
Lams veraon: 6.613 & Page 1 of 1 & $\mathbf{2 4}$ of $\mathbf{4 7}$
\end{tabular}




\section{Surrogate Summary for GC/MS Semi-volatiles}

Method SW8270SIMPAHD

Lab Name: ALS Environmental - FC

Work Order Number: 1209339

Client Name: Battelle Energy Alliance

ClientProject ID: MFC Biodiesel Tank Remova ,TOS-A1175 Rev2 BEA031231

\begin{tabular}{|c|c|c|c|}
\hline \multirow{2}{*}{$\begin{array}{l}\text { PrepBatchID: EX120921-6 } \\
\text { QC Batch ID: EX120921-6-1 }\end{array}$} & Surrogate Compound & \multicolumn{2}{|c|}{ Control Limits } \\
\hline & 2,4,6-Tribromophenol & & \\
\hline \multirow{5}{*}{ Date Extracted: $9 / 21 / 2012$} & 2-Fluorobiphenyl & 41 & 106 \\
\hline & 2-Fluorophend & & \\
\hline & Nitrobenzene-d5 & 28 & 113 \\
\hline & Phenol-d5 & & \\
\hline & Terphenyi-d14 & 25 & 147 \\
\hline
\end{tabular}

\begin{tabular}{|c|c|c|c|c|c|c|c|c|c|}
\hline Lab ID & Client Sample ID & $\begin{array}{c}\text { Date } \\
\text { Collected }\end{array}$ & $\begin{array}{c}\text { Date } \\
\text { Received }\end{array}$ & $\begin{array}{l}245 T B \% \\
\text { Recovery }\end{array}$ & $\begin{array}{c}2 F B P \% \\
\text { Recovery }\end{array}$ & $\begin{array}{c}2 F P \% \\
\text { Recovery }\end{array}$ & $\begin{array}{c}\text { ND5 \% } \\
\text { Recovery }\end{array}$ & $\begin{array}{c}\text { PD5 \% } \\
\text { Rөcovery }\end{array}$ & $\begin{array}{l}\text { TD14\% } \\
\text { Recovery }\end{array}$ \\
\hline $1209339-14$ & BEA031250_MFC & $9 / 2 \mathrm{D} / 2012$ & $9 / 21 / 2012$ & & 75 & & 82 & & 89 \\
\hline $1209339-18$ & BEA031254_MFC & $9 / 20 / 2012$ & $9 / 21 / 2012$ & & 73 & & 79 & & 92 \\
\hline
\end{tabular}

Date Printed: Tuesday, September 25, 2012 Shaded values exceed established control limits.
ALS Environmental -- FC LMS Verion: 6.613
Page 1 of 1

25 of 47 


\title{
GC/MS Semi-volatiles
}

\author{
Method SW8270SIMPAHD
}

Laboratory Control Sample and Laboratory Control Sample Duplicate

Lab Name: ALS Environmental - FC

Work Order Number: 1208339

Client Name: Battele Energy Alliance

ClientProject ID: MFC Biodiesel Tank Removal ,TOS-A1175 ReV2 BEA031231

\begin{tabular}{|c|c|c|c|c|}
\hline \multirow[t]{4}{*}{ Lab ID: EX120921-6LCS } & $\begin{array}{c}\text { Sample Matrix: SOIL } \\
\text { \% Moisture: N/A }\end{array}$ & $\begin{array}{l}\text { Prep Batch: EX120921-6 } \\
\text { QCBatchID: EX120921-6-1 }\end{array}$ & $\begin{array}{l}\text { Sample Aliquot: } \\
\text { Final Volume: }\end{array}$ & $\begin{array}{l}30 \mathrm{~g} \\
1 \mathrm{ml}\end{array}$ \\
\hline & Date Collected: N/A & Run ID: SV120922-1 & \multicolumn{2}{|c|}{ Result Units: UG/KG } \\
\hline & Date Analyzed: 09/22/2012 & Basis: N/A & & \\
\hline & Prep Method: SW3540C & File Name: N6034 & & \\
\hline
\end{tabular}

\begin{tabular}{|c|c|c|c|c|c|c|c|}
\hline CASNO & Target Analyte & $\begin{array}{l}\text { Spike } \\
\text { Added }\end{array}$ & $\begin{array}{l}\text { LCS } \\
\text { Result }\end{array}$ & $\begin{array}{l}\text { Reporting } \\
\text { Limit }\end{array}$ & $\begin{array}{l}\text { Result } \\
\text { Qualifier }\end{array}$ & $\begin{array}{l}\text { LCS \% } \\
\text { Rec. }\end{array}$ & $\begin{array}{c}\text { Control } \\
\text { Limits }\end{array}$ \\
\hline $91-20-3$ & NAPHTHALENE & 66.7 & 55.5 & 3.33 & & 83 & $40-107 \%$ \\
\hline $208-86-8$ & ACENAPHTHYLENE & 68.7 & 55.8 & 3.33 & & 84 & $44-107 \%$ \\
\hline $83-32-8$ & ACENAPHTHENE & 68.7 & 56 & 3.33 & & 84 & $46-108 \%$ \\
\hline $86-73-7$ & FLUORENE & 68.7 & 55.8 & 3.33 & & 84 & $49-108 \%$ \\
\hline $85-01-8$ & PHENANTHRENE & 68.7 & 55.4 & 3.33 & & 83 & $50-110 \%$ \\
\hline $120-12-7$ & ANTHRACENE & 68.7 & 57 & 3.33 & & 86 & $53-107 \%$ \\
\hline $206-44-0$ & FLUORANTHENE & 68.7 & 57.8 & 3.33 & & 87 & $54-114 \%$ \\
\hline $129-00-0$ & PYRENE & 66.7 & 44.3 & 3.33 & & 66 & $46-123 \%$ \\
\hline $56-55-3$ & BENZO(A)ANTHRACENE & 68.7 & 55 & 3.33 & & 82 & $52-111 \%$ \\
\hline $218-01-9$ & CHRYSENE & 66.7 & 55.3 & 3.33 & & 83 & $53-112 \%$ \\
\hline $205-80-2$ & BENZO(B)FLUORANTHENE & 66.7 & 60.2 & 3.33 & & 90 & $45-114 \%$ \\
\hline $207-08-9$ & BENZO(K)FLUORANTHENE & 68.7 & 53.4 & 3.33 & & 80 & $45-123 \%$ \\
\hline $50-32-8$ & BENZO(A)PYRENE & 68.7 & 59.7 & 3.33 & & 90 & $50-111 \%$ \\
\hline $193-39-5$ & INDENO $(1,2,3$-CD)PYRENE & 68.7 & 64.4 & 3.33 & & 97 & $38-121 \%$ \\
\hline $53-70-3$ & DIBENZO(A,H)ANTHRACENE & 66.7 & 71.4 & 3.33 & & 107 & $41-125 \%$ \\
\hline $191-24-2$ & BENZO(G,H,I)PERYLENE & 68.7 & 64.4 & 3.33 & & 97 & $38-126 \%$ \\
\hline
\end{tabular}

Data Package ID: SV1209339-1

Date Printed: Tuesday, September 25, 2012

ALS Environmental _- FC

uMs veraion: 6.613
Page 1 of 2

26 of 47 


\section{GC/MS Semi-volatiles}

\section{Method SW8270SIMPAHD}

Laboratory Control Sample and Laboratory Control Sample Duplicate

Lab Name: ALS Environmental - FC

Work Order Number: 1209339

Client Name: Battele Energy Alliance

ClientProject ID: MFC Biodiesel Tank Remova ,TOS-A1175 Rev2 BEA031231

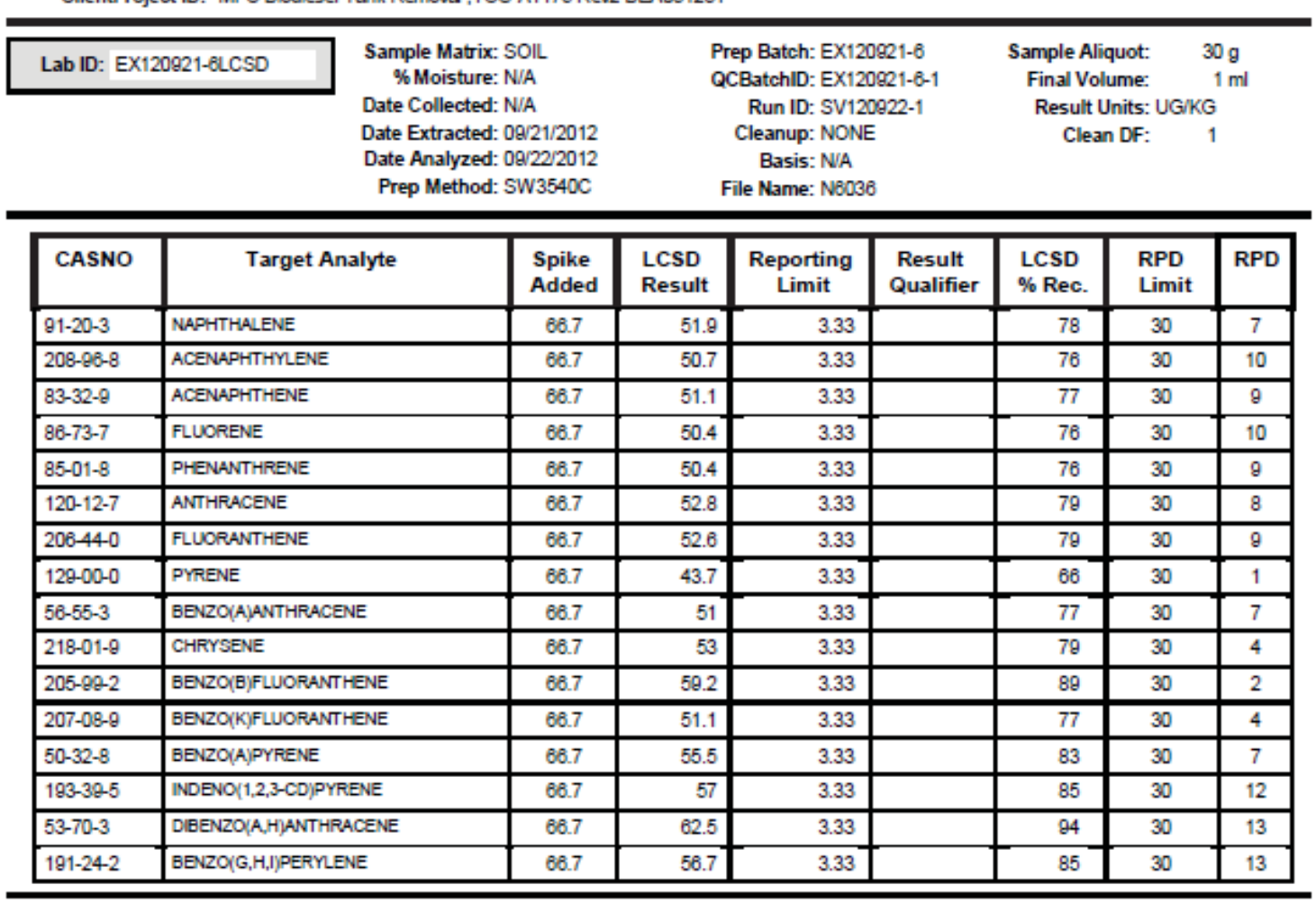

Surrogate Recovery LCS/LCSD

\begin{tabular}{|l|l|r|r|r|r|r|c|}
\hline CASNO & \multicolumn{1}{|c|}{ Target Analyte } & $\begin{array}{c}\text { Spike } \\
\text { Added }\end{array}$ & $\begin{array}{c}\text { LCS } \% \\
\text { Rec. }\end{array}$ & $\begin{array}{c}\text { LCS } \\
\text { Flag }\end{array}$ & $\begin{array}{c}\text { LCSD } \\
\% \text { Rec. }\end{array}$ & $\begin{array}{c}\text { LCSD } \\
\text { Flag }\end{array}$ & $\begin{array}{c}\text { Control } \\
\text { Limits }\end{array}$ \\
\hline $321-60-8$ & 2-FLUOROBIPHENYL & 66.7 & 78 & & 72 & & $41-106$ \\
\hline $4165-60-0$ & NTRROBENZENE-D5 & 66.7 & 85 & & 80 & & $28-113$ \\
\hline $1718-51-0$ & TERPHENYL-D14 & 66.7 & 75 & & 74 & & $25-147$ \\
\hline
\end{tabular}

Data Package ID: SV1209339-1

Date Printed: Tuesday, September 25, 2012

ALS Environmental - FC

Page 2 of 2

ums version: 6.613 


\section{GC/MS Semi-volatiles}

Method SW8270SIMPAHD

Matrix Spike And Matrix Spike Duplicate

Lab Name: ALS Environmental - FC

Work Order Number: 1209339

Client Name: Battelle Energy Alliance

ClientProject ID: MFC Biociesel Tank Removal ,TOS-A1175 Rev2 BEA03123

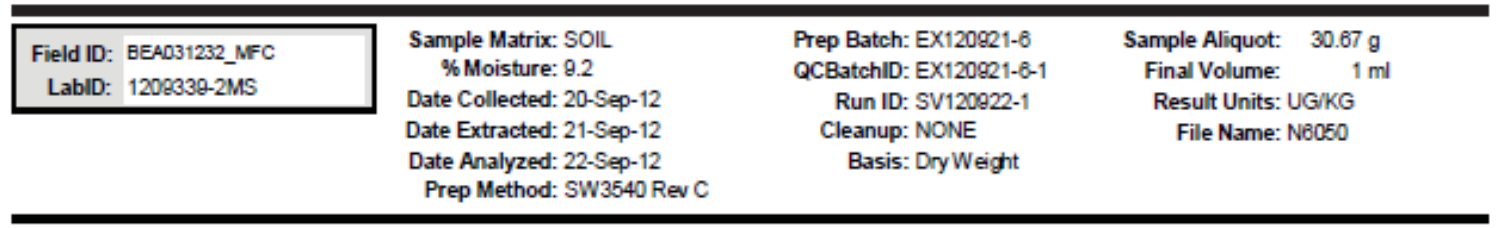

\begin{tabular}{|c|c|c|c|c|c|c|c|c|c|}
\hline CASNO & Target Analyte & $\begin{array}{l}\text { Sample } \\
\text { Result }\end{array}$ & $\begin{array}{r}\text { Samp } \\
\text { Qual }\end{array}$ & $\begin{array}{c}\text { MS } \\
\text { Result }\end{array}$ & $\begin{array}{l}\text { MS } \\
\text { Qual }\end{array}$ & $\begin{array}{l}\text { Reporting } \\
\text { Limit }\end{array}$ & $\begin{array}{l}\text { Spike } \\
\text { Added }\end{array}$ & $\begin{array}{c}\text { MS \% } \\
\text { Rec. }\end{array}$ & $\begin{array}{c}\text { Control } \\
\text { Limits }\end{array}$ \\
\hline $91-20-3$ & NAPHTHALENE & 3.6 & $u$ & 48.8 & & 3.59 & 71.8 & 68 & $40-107 \%$ \\
\hline $208-96-8$ & ACENAPHTHYLENE & 3.6 & $\bar{U}$ & 49.1 & & 3.59 & 71.8 & 68 & $44-107 \%$ \\
\hline $83-32-8$ & ACENAPHTHENE & 3.6 & u & 51.4 & & 3.59 & 71.8 & 72 & $46-108 \%$ \\
\hline $86-73-7$ & FLUORENE & 3.6 & $\bar{U}$ & 52.1 & & 3.59 & 71.8 & 73 & $49-108 \%$ \\
\hline $85-01-8$ & PHENANTHRENE & 9.6 & & 53 & & 3.59 & 71.8 & 60 & $50-110 \%$ \\
\hline $120-12-7$ & ANTHRACENE & 3.2 & $\mathrm{~J}$ & 52.4 & & 3.59 & 71.8 & 69 & $53-107 \%$ \\
\hline $206-44-0$ & FLUORANTHENE & 88 & & 78.5 & $\therefore$ & 3.59 & 71.8 & 17 & $54-114 \%$ \\
\hline $128-00-0$ & PYRENE & 61 & & 73.4 & $\mathrm{x}$ & 3.59 & 71.8 & 18 & $46-123 \%$ \\
\hline $56-55-3$ & BENZO(A)ANTHRACENE & 44 & & 60 & $\bar{x}$ & 3.59 & 71.8 & 34 & $52-111 \%$ \\
\hline $218-01-\theta$ & CHRYSENE & 42 & & 73 & $\mathrm{x}$ & 3.59 & 71.8 & 43 & $53-112 \%$ \\
\hline $205-9 \theta-2$ & BENZO(B)FLUORANTHENE & $9 \theta$ & & 114 & $\pi$ & 3.59 & 71.8 & 21 & $45-114 \%$ \\
\hline $207-08-\theta$ & BENZO(K)FLUORANTHENE & 40 & & 81.5 & & 3.59 & 71.8 & 58 & $45-123 \%$ \\
\hline $50-32-8$ & BENZO(A)PYFENE & 60 & & 81.3 & 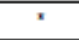 & 3.59 & 71.8 & 30 & $50-111 \%$ \\
\hline $193-39-5$ & INDENO(1,2,3-CD)PYRENE & 25 & & 51.6 & $\therefore$ & 3.59 & 71.8 & 37 & $38-121 \%$ \\
\hline $53-70-3$ & DIEENZO(A,H)ANTHRACENE & 9.3 & & 50.8 & & 3.59 & 71.8 & 58 & $41-125 \%$ \\
\hline $191-24-2$ & BENZO(G,H,I)PERYLENE & 30 & & 50.2 & 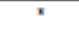 & 3.59 & 71.8 & 28 & $38-126 \%$ \\
\hline
\end{tabular}

Data Package ID: SV1209339-1

$\begin{array}{lcl}\text { Date Printed: Tuesday, September 25, } 2012 & \begin{array}{c}\text { ALS Environmental -- FC } \\ \text { LMs version: 6.513 }\end{array} & \text { Page } 1 \text { of } 2\end{array}$




\section{GC/MS Semi-volatiles}

Method SW8270SIMPAHD

Matrix Spike And Matrix Spike Duplicate

Lab Name: ALS Environmental - FC

Work Order Number: 1209339

Client Name: Battelle Energy Alliance

ClientProject ID: MFC Biociesel Tank Removal ,TOS-A1175 Rev2 BEA03123

\begin{tabular}{|c|c|c|c|}
\hline $\begin{array}{r}\text { Field ID: } \text { 日EA031232_MFC } \\
\text { LabID: } 1208339-2 \text { MSD } \\
\end{array}$ & $\begin{array}{l}\text { Sample Matrix: SOIL } \\
\quad \text { \%oisture: } 9.2 \\
\text { Date Collected: } 20 \text {-Sep-12 }\end{array}$ & $\begin{array}{l}\text { Prep Batch: EX120921-6 } \\
\text { QCBatchID: EX120921-6-1 } \\
\text { Run ID: SV120922-1 }\end{array}$ & $\begin{array}{cc}\text { Sample Aliquot: } & 30.14 \mathrm{~g} \\
\text { Final Volume: } & 1 \mathrm{ml} \\
\text { Result Units: UG/KG }\end{array}$ \\
\hline & Date Extracted: 21-Sep-12 & Cleanup: NONE & File Name: N6051 \\
\hline & $\begin{array}{l}\text { Date Analyzed: } 22-\text { Sep-12 } \\
\text { Prep Method: SW3540 Rev C }\end{array}$ & Basis: DryWeight & \\
\hline
\end{tabular}

\begin{tabular}{|c|c|c|c|c|c|c|c|c|}
\hline CASNO & Target Analyte & $\begin{array}{l}\text { MSD } \\
\text { Result }\end{array}$ & $\begin{array}{l}\text { MSD } \\
\text { Qual }\end{array}$ & $\begin{array}{l}\text { Spike } \\
\text { Added }\end{array}$ & $\begin{array}{c}\text { MSD \% } \\
\text { Rec. }\end{array}$ & $\begin{array}{l}\text { Reporting } \\
\text { Limit }\end{array}$ & $\begin{array}{l}\text { RPD } \\
\text { Limit }\end{array}$ & RPD \\
\hline $91-20-3$ & NAPHTHALENE & 53.3 & & 73 & 73 & 3.65 & 30 & 9 \\
\hline $208-96-8$ & ACENAPHTHYLENE & 56.7 & & 73 & 76 & 3.65 & 30 & 13 \\
\hline $83-32-\theta$ & ACENAPHTHENE & 58.1 & & 73 & 79 & 3.65 & 30 & 12 \\
\hline $86-73-7$ & FLUORENE & 57.5 & & 73 & 79 & 3.65 & 30 & 10 \\
\hline $85-01-8$ & PHENANTHRENE & 60.1 & & 73 & 69 & 3.65 & 30 & 13 \\
\hline $120-12-7$ & ANTHRACENE & 50.2 & & 73 & 77 & 3.65 & 30 & 12 \\
\hline $206-44-0$ & FLUORANTHENE & 89.8 & . & 73 & 32 & 3.65 & 30 & 14 \\
\hline $129-00-0$ & PYRENE & 89 & 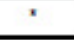 & 73 & 39 & 3.65 & 30 & 19 \\
\hline $56-55-3$ & BENZO(A)ANTHRACENE & 79.8 & 5 & 73 & 49 & 3.65 & 30 & 14 \\
\hline $218-01-8$ & CHRYSENE & 78.7 & 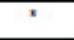 & 73 & 50 & 3.65 & 30 & 7 \\
\hline $205-99-2$ & BENZO(B)FLUORANTHENE & 126 & 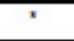 & 73 & 38 & 3.65 & 30 & 11 \\
\hline $207-08-8$ & BENZO(K)FLUORANTHENE & 104 & & 73 & 88 & 3.65 & 30 & 24 \\
\hline $50-32-8$ & BENZO(A)PYRENE & 82.7 & 5 & 73 & 45 & 3.65 & 30 & 13 \\
\hline $193-32-5$ & INDENO $(1,2,3-C D)$ PYRENE & 56.4 & & 73 & 43 & 3.65 & 30 & 9 \\
\hline $53-70-3$ & DIEENZO(A,H)ANTHRACENE & 56.7 & & 73 & 64 & 3.65 & 30 & $\theta$ \\
\hline $191-24-2$ & BENZO(G,H,I)PERYLENE & 53.3 & $\bar{s}$ & 73 & 31 & 3.65 & 30 & 6 \\
\hline
\end{tabular}

Surrogate Recovery MS/MSD

\begin{tabular}{|l|l|r|r|r|r|r|c|}
\hline CASNO & \multicolumn{1}{|c|}{ Target Analyte } & $\begin{array}{c}\text { Spike } \\
\text { Added }\end{array}$ & $\begin{array}{c}\text { MS \% } \\
\text { Rec. }\end{array}$ & $\begin{array}{c}\text { MS } \\
\text { Flag }\end{array}$ & $\begin{array}{c}\text { MSD \% } \\
\text { Rec. }\end{array}$ & $\begin{array}{c}\text { MSD } \\
\text { Flag }\end{array}$ & $\begin{array}{c}\text { Control } \\
\text { Limits }\end{array}$ \\
\hline $321-60-8$ & 2-FLUOROEIPHENYL & 71.8 & 68 & & 71 & & $41-106$ \\
\hline $4165-60-0$ & NITROEENLENE-D5 & 71.8 & 71 & & 76 & & $28-113$ \\
\hline $1718-51-0$ & TERPHENYL-D14 & 71.8 & 80 & & 94 & & $25-147$ \\
\hline
\end{tabular}

Data Package ID: SV1209339-1

\begin{tabular}{lcc}
\hline Date Printed: Tuesday, September 25, 2012 & ALS Environmental -- FC \\
Lms version: 6.513 & Page 2 of 2
\end{tabular}




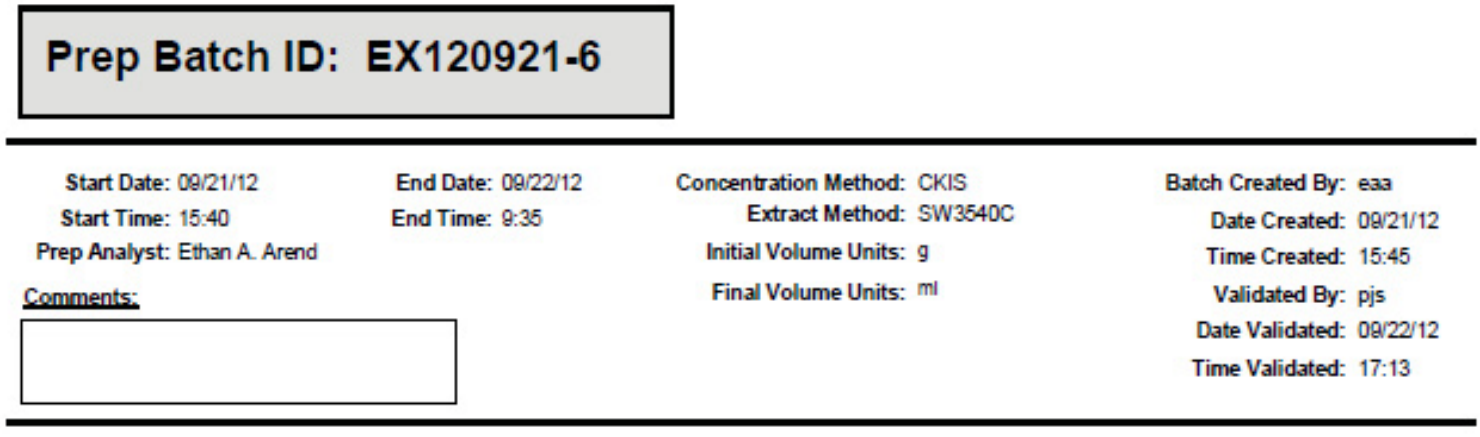

QC Batch ID: EX120921-6-1

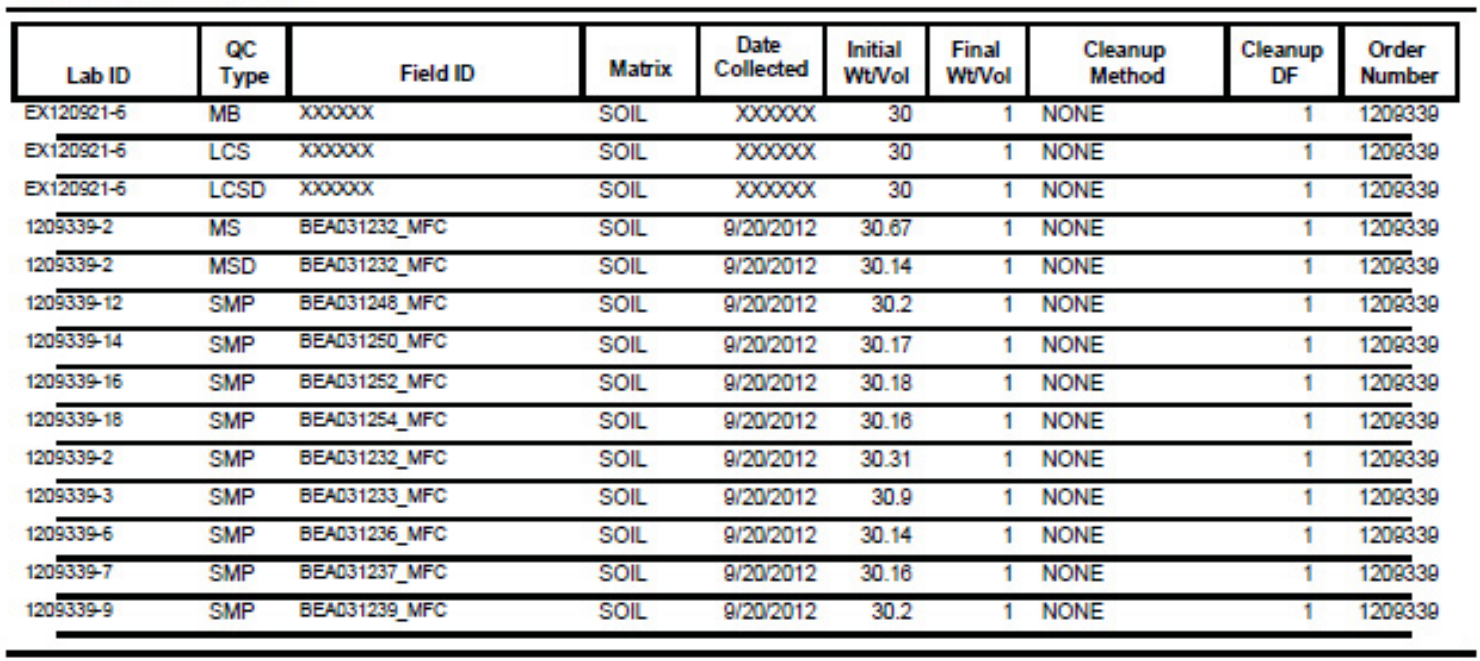

In generating this benchsheet, prep analyst states that al aspects of sample preparation as set forth in the appropriate SOP's (inciuding KudemaDanish temperatures, proper flow settings on the $\mathrm{N}$-evap, and final volumes) were properiy adhered to (unless otherwise noted herein).

\section{QC.Trpes}

\begin{tabular}{|l|l|}
\hline CAR & Carrler reference sample \\
\hline LCS & Laboratory Control Sample \\
\hline MB & Method Blank \\
\hline MSD & Laboratory Matrix Splke Dupllcate \\
\hline RVS & Reporting Level Veriflcation Standar \\
\hline SYS & Sample Yeld Splke \\
\hline
\end{tabular}

\begin{tabular}{|l|l|}
\hline DUP & Laboratory Duplicate \\
\hline LCSD & Laboratory Control Sample Duplicat \\
\hline MS & Laboratory Matrix Splke \\
\hline REP & Sample replicate \\
\hline SMP & Fleld Sample \\
\hline
\end{tabular}




\section{B}

Semi-Volatile Organic GC/MS Tuning And Mass

Calibration--Decafluorotriphenylphosphine (DFTPP)

Lab Name: ALS Environmental - FC Work Order Number: 1202339

Client Name: Battelle Energy Alliance

ClientProject ID: BEA031231MFC Biodiesel Tank Removal ,TOS-A1175 Rev2
DFTPP Injection Date: $\quad 8 / 20 / 2012$

DFTPP Injection Time: $\quad 9: 27$

Instrument ID: HPSV1

Reported on: Tuesday. September 25, 2012

FilelD: $N 6000$

\begin{tabular}{|l|l|r|}
\hline mie & $\begin{array}{c}\text { lon Abundance Criteria } \\
\text { SW8270SIMPAHD }\end{array}$ & $\begin{array}{c}\text { \% Relative } \\
\text { Abundance }\end{array}$ \\
\hline 51 & $30.0-60.0$ percent of mass 188 & 51.7 \\
\hline 68 & Less than 2.0 percent of mass 69 & 0 \\
\hline 69 & Mass 69 relative abundance of mass 198 & 52.9 \\
\hline 70 & Less than 2.0 percent of mass 69 & 0 \\
\hline 127 & $40.0-60.0$ percent of mass 198 & 58.8 \\
\hline 197 & Less than 1.0 percent of mass 198 & 0 \\
\hline 198 & Base peak, 100 percent of relative abundance & 100 \\
\hline 199 & $5.0-9.0$ percent of mass 198 & 6.9 \\
\hline 275 & $10.0-30.0$ percent of mass 198 & 28.6 \\
\hline 365 & Greater than 1.00 percent of mass 188 & 3.4 \\
\hline 441 & Present, but less than mass 443 (percent of 443 ) & 73.1 \\
\hline 442 & Greater than 40.0 percent of mass 188 & 79.8 \\
\hline 443 & $17.0-23.0$ percent of mass 442 & 19.3 \\
\hline
\end{tabular}

THIS TUNE APPLIES TO THE FOLLOWING SAMPLES, MS/MSD, BLANKS, AND STANDARDS:

\begin{tabular}{|c|c|c|c|c|c|}
\hline Client Sample ID & Lab Sample ID & Lab File ID & $\begin{array}{c}\text { Date } \\
\text { Analyzed }\end{array}$ & $\begin{array}{c}\text { Time } \\
\text { Analyzed }\end{array}$ & QC BatchID \\
\hline $2000000 x$ & ICALSVSTD0500CSTD & N6001 & $9 / 20 / 2012$ & $10-02$ & SV120e20-1 \\
\hline $2000000 x$ & ICALSVSTD0050CSTD & N6002 & $9 / 20 / 2012$ & $10: 23$ & SV120820-1 \\
\hline $2000000 x$ & ICALSVSTD0100CSTD & N6003 & $9 / 20 / 2012$ & $10: 44$ & SV120920-1 \\
\hline $2000000 x$ & ICALSVSTD0200CSTD & N6004 & $9 / 20 / 2012$ & $11: 04$ & SV120920-1 \\
\hline$x 000000 x$ & ICALSVSTD1000CSTD & N6005 & $9 / 20 / 2012$ & $11: 25$ & SV120920-1 \\
\hline $2000000 x$ & ICALSVSTD2000CSTD & N6006 & $9 / 20 / 2012$ & $11: 45$ & SV120820-1 \\
\hline $1000000 X$ & ICALSVSTD5000CSTD & N6007 & $9 / 20 / 2012$ & $12-06$ & SV120920-1 \\
\hline $2000000 x$ & ICVSVSTD2000ICV & N6009 & $9 / 20 / 2012$ & $13: 11$ & SV120920-1 \\
\hline $1000000 x$ & $1209035-14$ & N6011 & $9 / 20 / 2012$ & $14: 14$ & EX120915-2-1 \\
\hline$x 000000 x$ & $1209035-17$ & N6013 & $9 / 20 / 2012$ & 14.55 & EX120915-2-1 \\
\hline $1000000 X$ & $1209035-17 \mathrm{MS}$ & N6014 & $9 / 20 / 2012$ & $15: 18$ & EX120915-2-1 \\
\hline $1000000 x$ & $1209035-17 \mathrm{MSD}$ & N6015 & $9 / 20 / 2012$ & $15: 43$ & EX120915-2-1 \\
\hline $2000000 x$ & $1209035-1$ & N6016 & $9 / 20 / 2012$ & $16: 08$ & EX120915-2-1 \\
\hline
\end{tabular}

Data Package ID: SV1209339-1

Date Printed: Tuesday, September 25, 2012

ALS Environmental - FC LaMs veraion: 6.513
Page 1 of 2

31 of 47 


\section{5в}

Semi-Volatile Organic GC/MS Tuning And Mass

Calibration--Decafluorotriphenylphosphine (DFTPP)

Lab Name: ALS Environmental - FC

DFTPP Injection Date: $\quad$ 8/20/2012

Work Order Number: 1208339

Client Name: Battelle Energy Alliance

DFTPP Injection Time: $\quad 9: 27$

Instrument ID: HPSV1

ClientProject ID: BEA031231MFC Biodiesel Tank Removal ,TOS-A1175 Rev2

Reported on: Tuesday. September 25, 2012

FilelD: $N 6000$

\begin{tabular}{|l|l|r|}
\hline mie & $\begin{array}{c}\text { lon Abundance Criteria } \\
\text { SW8270SIMPAHD }\end{array}$ & $\begin{array}{c}\text { \% Relative } \\
\text { Abundance }\end{array}$ \\
\hline 51 & $30.0-60.0$ percent of mass 198 & 51.7 \\
\hline 68 & Less than 2.0 percent of mass 69 & 0 \\
\hline 69 & Mass 69 relative abundance of mass 198 & 52.8 \\
\hline 70 & Less than 2.0 percent of mass 69 & 0 \\
\hline 127 & $40.0-60.0$ percent of mass 198 & 58.8 \\
\hline 197 & Less than 1.0 percent of mass 198 & 0 \\
\hline 198 & Base peak, 100 percent of relative abundance & 100 \\
\hline 199 & $5.0-9.0$ percent of mass 198 & 6.8 \\
\hline 275 & $10.0-30.0$ percent of mass 198 & 28.6 \\
\hline 365 & Greater than 1.00 percent of mass 188 & 3.4 \\
\hline 441 & Present, but less than mass 443 (percent of 443 ) & 73.1 \\
\hline 442 & Greater than 40.0 percent of mass 188 & 79.8 \\
\hline 443 & $17.0-23.0$ percent of mass 442 & 19.3 \\
\hline
\end{tabular}

THIS TUNE APPLIES TO THE FOLLOWING SAMPLES, MS/MSD, BLANKS, AND STANDARDS:

\begin{tabular}{|c|c|c|c|c|c|}
\hline Client Sample ID & Lab Sample ID & Lab File ID & $\begin{array}{c}\text { Date } \\
\text { Analyzed }\end{array}$ & $\begin{array}{c}\text { Time } \\
\text { Analyzed }\end{array}$ & QC BatchID \\
\hline $2000000 x$ & $1209035-12$ & N6017 & $9 / 20 / 2012$ & $16: 32$ & EX120915-2-1 \\
\hline 10000000 & $1209035-6$ & N6018 & $9 / 20 / 2012$ & 16.55 & EX120915-2-1 \\
\hline $1000000 X$ & 1209035-14RR1 & N6019 & $8 / 20 / 2012$ & $17: 17$ & EX120915-2-1 \\
\hline $2000000 x$ & $1209035-16$ & $\mathrm{~N} 6020$ & $9 / 20 / 2012$ & $17: 47$ & EX120915-2-1 \\
\hline$x 000000 x$ & $1209035-8$ & $\mathrm{~N} 6021$ & $9 / 20 / 2012$ & $18: 07$ & EX120915-2-1 \\
\hline 10000000 & $1209035-4$ & N6022 & $9 / 20 / 2012$ & $18: 28$ & EX120915-2-1 \\
\hline $2000000 x$ & $1209035-3$ & $\mathrm{~N} 6023$ & $9 / 20 / 2012$ & $18: 48$ & EX120915-2-1 \\
\hline 10000000 & $1209035-9$ & N6024 & $9 / 20 / 2012$ & $19: 00$ & EX120915-2-1 \\
\hline $2000000 x$ & $1209035-11$ & N6025 & $9 / 20 / 2012$ & $19: 30$ & EX120915-2-1 \\
\hline 10000000 & $1209035-15$ & N6028 & $8 / 20 / 2012$ & 19.51 & EX120915-2-1 \\
\hline $2000000 x$ & $1209035-2$ & N6027 & $9 / 20 / 2012$ & $20: 11$ & EX120915-2-1 \\
\hline $1000000 X$ & $1209035-10$ & N6028 & $8 / 20 / 2012$ & $20: 32$ & EX120915-2-1 \\
\hline $1000000 x$ & $1209035-13$ & N6029 & $9 / 20 / 2012$ & 20.53 & EX120915-2-1 \\
\hline
\end{tabular}

Data Package ID: SV1209339-1

Date Printed: Tuesday, September 25, 2012

ALS Environmental - FC

Page 2 of 2

Las veraon: 6.513

32 of 47 


\section{B}

Semi-Volatile Organic GC/MS Tuning And Mass

Calibration--Decafluorotriphenylphosphine (DFTPP)

Lab Name: ALS Environmental - FC Work Order Number: 1202339

Client Name: Battele Energy Alliance

ClientProject ID: BEA031231MFC Biodiesel Tank Removal ,TOS-A1175 Rev2
DFTPP Injection Date: DFTPP Injection Time: Instrument ID:

Fileld: $N 6031$

\begin{tabular}{|c|l|c|}
\hline mie & $\begin{array}{c}\text { lon Abundance Criteria } \\
\text { SW8270SIMPAHD }\end{array}$ & $\begin{array}{c}\text { \%Relative } \\
\text { Abundance }\end{array}$ \\
\hline 51 & $30.0-60.0$ percent of mass 198 & 50.1 \\
\hline 68 & Less than 2.0 percent of mass 69 & 0 \\
\hline 69 & Mass 69 relative abundance of mass 198 & 50.2 \\
\hline 70 & Less than 2.0 percent of mass 69 & 0 \\
\hline 127 & $40.0-60.0$ percent of mass 198 & 51.8 \\
\hline 197 & Less than 1.0 percent of mass 198 & 0 \\
\hline 198 & Base peak 100 percent of relative abundance & 100 \\
\hline 199 & $5.0-9.0$ percent of mass 198 & 6.6 \\
\hline 275 & $10.0-30.0$ percent of mass 198 & 28.4 \\
\hline 365 & Greater than 1.00 percent of mass 188 & 3.2 \\
\hline 441 & Present, but less than mass 443 (percent of 443 ) & 33.6 \\
\hline 442 & Greater than 40.0 percent of mass 188 & 68.5 \\
\hline 443 & $17.0-23.0$ percent of mass 442 & 19.3 \\
\hline
\end{tabular}

THIS TUNE APPLIES TO THE FOLLOWING SAMPLES, MS/MSD, BLANKS, AND STANDARDS:

\begin{tabular}{|c|c|c|c|c|c|}
\hline Client Sample ID & Lab Sample ID & Lab File ID & $\begin{array}{c}\text { Date } \\
\text { Analyzed }\end{array}$ & $\begin{array}{c}\text { Time } \\
\text { Analyzed }\end{array}$ & QC BatchID \\
\hline $1000000 X$ & $\mathrm{CCV} 1 \mathrm{CCV}$ & N6032 & $9 / 22 / 2012$ & $17: 09$ & SV120922-1 \\
\hline$x 000000 x$ & EX120921-6MB & N6033 & $8 / 22 / 2012$ & $17: 30$ & EX120e21-6-1 \\
\hline $0000000 x$ & EX120021-6LCS & N6034 & $9 / 22 / 2012$ & $17: 50$ & EX120e21-6-1 \\
\hline$x 000000 x$ & EX120921-6LCSD & N6038 & $9 / 22 / 2012$ & $18: 31$ & EX120e21-6-1 \\
\hline BEAD31252_MFC & $1208339-16$ & N6037 & $9 / 22 / 2012$ & 18.51 & EX120e21-6-1 \\
\hline BEAD31252_MFC & 1208339-16RR1 & N6047 & $9 / 22 / 2012$ & $22: 15$ & EX120e21-6-1 \\
\hline BEAD31237_MFC & $1209339-7$ & N6048 & $9 / 22 / 2012$ & $22: 35$ & EX120e21-6-1 \\
\hline BEAD31232_MFC & $1209339-2$ & N6049 & $9 / 22 / 2012$ & $22-56$ & EX120e21-6-1 \\
\hline BEAD31232_MFC & $1208339-2 M S$ & N6050 & $9 / 22 / 2012$ & $23: 16$ & EX120e21-6-1 \\
\hline BEAD31232_MFC & $1208339-2 M S D$ & N6051 & $9 / 22 / 2012$ & $23: 36$ & EX120e21-6-1 \\
\hline BEAD31233_MFC & $1209339-3$ & N6052 & $9 / 22 / 2012$ & 23.57 & EX120e21-6-1 \\
\hline BEAD31236_MFC & $1208330-6$ & N6053 & $9 / 23 / 2012$ & $0: 17$ & EX120e21-6-1 \\
\hline BEAD31239_MFC & $1209339-9$ & N6054 & $9 / 23 / 2012$ & $0: 38$ & EX120e21-6-1 \\
\hline
\end{tabular}

Data Package ID: SV1209339-1

Date Printed: Tuesday, September 25, 2012

ALS Environmental - FC

$$
\text { LaMs verzion: } 6.613
$$

Page 1 of 2

33 of 47 


\section{B}

Semi-Volatile Organic GC/MS Tuning And Mass

Calibration--Decafluorotriphenylphosphine (DFTPP)

Lab Name: ALS Environmental - FC

Work Order Number: 1208339

Client Name: Battelle Energy Alliance

ClientProject ID: BEA031231MFC Biodiesel Tank Remova ,TOS-A1175 Rev2
DFTPP Injection Date: $\quad$ 8/22/2012

DFTPP Injection Time: $\quad$ 16:55

Instrument ID: HPSV1

Reported on: Tuesday. September 25, 2012

\begin{tabular}{|l|l|r|}
\hline mie & $\begin{array}{c}\text { lon Abundance Criteria } \\
\text { SW8270SIMPAHD }\end{array}$ & $\begin{array}{c}\text { \% Relative } \\
\text { Abundance }\end{array}$ \\
\hline 51 & $30.0-60.0$ percent of mass 198 & 50.1 \\
\hline 68 & Less than 2.0 percent of mass 69 & 0 \\
\hline 69 & Mass 69 relative abundance of mass 198 & 50.2 \\
\hline 70 & Less than 2.0 percent of mass 69 & 0 \\
\hline 127 & $40.0-60.0$ percent of mass 198 & 51.8 \\
\hline 197 & Less than 1.0 percent of mass 198 & 0 \\
\hline 198 & Base peak, 100 percent of relative abundance & 100 \\
\hline 199 & $5.0-9.0$ percent of mass 198 & 6.6 \\
\hline 275 & $10.0-30.0$ percent of mass 198 & 26.4 \\
\hline 365 & Greater than 1.00 percent of mass 188 & 3.2 \\
\hline 441 & Present, but less than mass 443 (percent of 443 ) & 33.6 \\
\hline 442 & Greater than 40.0 percent of mass 188 & 68.5 \\
\hline 443 & $17.0-23.0$ percent of mass 442 & 19.3 \\
\hline
\end{tabular}

THIS TUNE APPLIES TO THE FOLLOWING SAMPLES, MS/MSD, BLANKS, AND STANDARDS:

\begin{tabular}{|l|c|c|c|c|c|}
\hline \multicolumn{1}{|c|}{ Client Sample ID } & Lab Sample ID & Lab File ID & $\begin{array}{c}\text { Date } \\
\text { Analyzed }\end{array}$ & $\begin{array}{c}\text { Time } \\
\text { Analyzed }\end{array}$ & QC BatchID \\
\hline BEA031248_MFC & $1208339-12$ & N6056 & $9 / 23 / 2012$ & $0: 58$ & EX120821-6-1 \\
\hline
\end{tabular}

Date Printed: Tuesday, September 25, $2012 \quad$ ALS Environmental - FC
LMB veraion: 6.513
Page 2 of 2

34 of 47 


\section{$5 B$}

Semi-Volatile Organic GC/MS Tuning And Mass

Calibration--Decafluorotriphenylphosphine (DFTPP)

Lab Name: ALS Environmental - FC

Work Order Number: 1202339

Client Name: Battelle Energy Alliance

ClientProject ID: BEA031231MFC Biodiesel Tank Removal ,TOS-A1175 Rev2 $\begin{array}{rr}\text { DFTPP Injection Date: } \quad \text { 8/24/2012 } \\ \text { DFTPP Injection Time: } & 11: 06 \\ \text { Instrument ID: } & \text { HPSV1 }\end{array}$

Reported on: Tuesday. September 25, 2012

Fileld: $N 6059$

\begin{tabular}{|c|l|c|}
\hline mee & $\begin{array}{c}\text { lon Abundance Criteria } \\
\text { SW8270SIMPAHD }\end{array}$ & $\begin{array}{c}\text { \% Relative } \\
\text { Abundance }\end{array}$ \\
\hline 51 & $30.0-60.0$ percent of mass 198 & 53.8 \\
\hline 68 & Less than 2.0 percent of mass 69 & 0 \\
\hline 69 & Mass 69 relative abundance of mass 198 & 52.8 \\
\hline 70 & Less than 2.0 percent of mass 69 & 0 \\
\hline 127 & $40.0-60.0$ percent of mass 198 & 54.4 \\
\hline 197 & Less than 1.0 percent of mass 198 & 0 \\
\hline 198 & Base peak 100 percent of relative abundance & 100 \\
\hline 199 & $5.0-9.0$ percent of mass 198 & 6.7 \\
\hline 275 & $10.0-30.0$ percent of mass 198 & 28.3 \\
\hline 365 & Greater than 1.00 percent of mass 188 & 2.7 \\
\hline 441 & Present, but less than mass 443 (percent of 443 ) & 43 \\
\hline 442 & Greater than 40.0 percent of mass 188 & 64 \\
\hline 443 & $17.0-23.0$ percent of mass 442 & 19.6 \\
\hline
\end{tabular}

THIS TUNE APPLIES TO THE FOLLOWING SAMPLES, MS/MSD, BLANKS, AND STANDARDS:

\begin{tabular}{|c|c|c|c|c|c|}
\hline Client Sample ID & Lab Sample ID & Lab File ID & $\begin{array}{c}\text { Date } \\
\text { Analyzed }\end{array}$ & $\begin{array}{c}\text { Time } \\
\text { Analyzed }\end{array}$ & QC BatchID \\
\hline$x 000000 x$ & ICALSVSTD0500CSTD & N6060 & $8 / 24 / 2012$ & $11: 19$ & SV120924-1 \\
\hline $2000000 X$ & ICALSVSTD0050CSTD & N6081 & $9 / 24 / 2012$ & $12: 15$ & SV120924-1 \\
\hline$x 000000 x$ & ICALSVSTD0100CSTD & N6062 & $9 / 24 / 2012$ & $12: 35$ & SV120924-1 \\
\hline$x 000000 x$ & ICALSVSTD0200CSTD & $\mathrm{N} 6063$ & $9 / 24 / 2012$ & $12-55$ & SV120924-1 \\
\hline$x 000000 x$ & ICALSVSTD1000CSTD & N6084 & $9 / 24 / 2012$ & $13: 16$ & SV120924-1 \\
\hline $1000000 x$ & ICALSVSTD2000CSTD & N6085 & $8 / 24 / 2012$ & $13: 36$ & SV120924-1 \\
\hline $2000000 x$ & ICALSVSTD5000CSTD & N6068 & $9 / 24 / 2012$ & 13.56 & SV120924-1 \\
\hline $1000000 X$ & ICVSVSTD2000ICV & N6087 & $9 / 24 / 2012$ & $14: 17$ & SV120924-1 \\
\hline $2000000 x$ & EX120920-4MB & N6069 & $9 / 24 / 2012$ & 14.57 & EX120e20-4-1 \\
\hline $1000000 X$ & EX120920-4LCS & N6070 & $8 / 24 / 2012$ & $15: 18$ & EX120e20-4-1 \\
\hline $2000000 x$ & EX120920-4LCSD & N6071 & $9 / 24 / 2012$ & $15: 38$ & EX120e20-4-1 \\
\hline BEAD31250_MFC & $1209339-14$ & N6072 & $9 / 24 / 2012$ & $15: 58$ & EX120021-6-1 \\
\hline BEAD31254_MFC & $1208339-18$ & N6073 & $9 / 24 / 2012$ & $16: 21$ & EX120e21-6-1 \\
\hline
\end{tabular}

Data Package ID: SV1209339-1

Data Package

Date Printed: Tuesday, September 25, 2012

ALS Environmental - FC

LMS veraion: 6.613
Page 1 of 2

35 of 47 


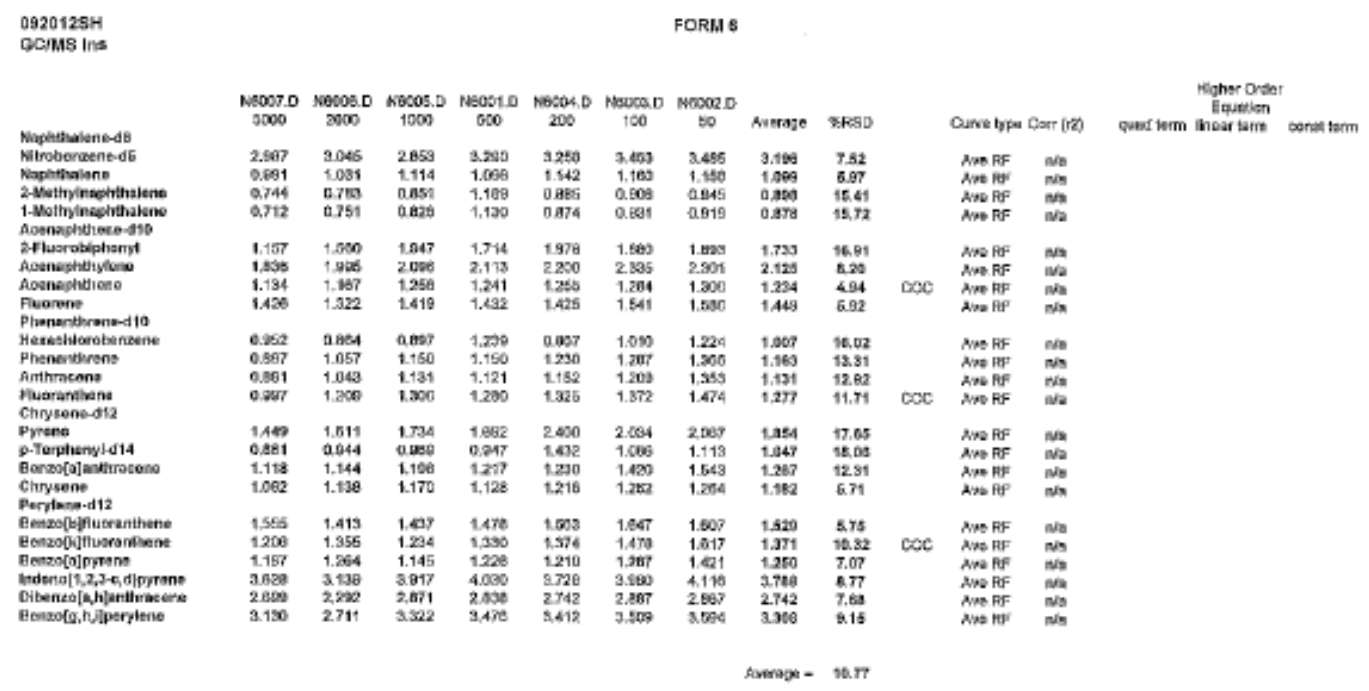

$g u$ $9-20-12$ 


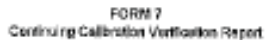

\begin{tabular}{|c|c|c|}
\hline & & 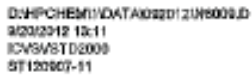 \\
\hline \multirow{2}{*}{\multicolumn{2}{|c|}{$\begin{array}{l}\text { Yetsod } \\
\text { Tile } \\
\text { Led Upet }\end{array}$}} & gasotzen \\
\hline & & 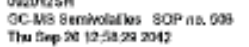 \\
\hline & & Corrpourd \\
\hline & STD & 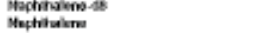 \\
\hline & & 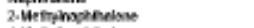 \\
\hline & & 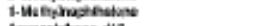 \\
\hline & 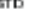 & Acomplitanedid \\
\hline & $x$ & Nomaptitime \\
\hline$n$ & & Fluseren \\
\hline ?] & GTD & 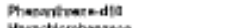 \\
\hline 2 & & Hewacticrobsaztio \\
\hline 13) & & Fiverantives: \\
\hline 10 & & Artrocense \\
\hline 5] & 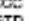 & Flariatione \\
\hline 5) & & Carysem-412 \\
\hline is & & Preis \\
\hline 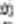 & & 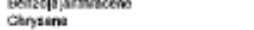 \\
\hline 10 & STD & Perienest2 \\
\hline ख] & & 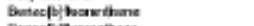 \\
\hline $2 x$ & & mascisjomarteno \\
\hline 26) & 20 & 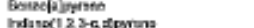 \\
\hline 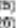 & & 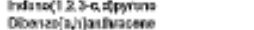 \\
\hline & & 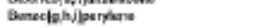 \\
\hline
\end{tabular}

\begin{tabular}{|c|c|c|c|c|c|c|c|}
\hline \multirow{3}{*}{$\frac{\operatorname{tog} R F}{1000}$} & \multirow[b]{3}{*}{$\frac{\mathrm{cces}}{20 m}$} & \multirow[b]{3}{*}{ Eutcons } & \multirow{3}{*}{ Paunderero } & \multirow{3}{*}{$\begin{array}{l}\text { \$Dovar } \\
\text { Sonen }\end{array}$} & \multirow{3}{*}{ 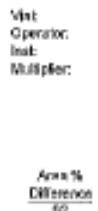 } & \multicolumn{2}{|c|}{ 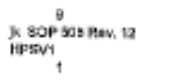 } \\
\hline & & & & & & R.T.Dor & Cune fil \\
\hline & & & & & & 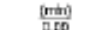 & $\frac{\operatorname{TMP}}{A 10}$ \\
\hline 1 098 & 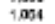 & & & -12 & 175 & 0,0 & Ane ne \\
\hline 0.893 & 0.794 & & & 127 & 10 & 00 & Ale $18=$ \\
\hline $\mathrm{d} / \mathrm{s} / \mathrm{s}$ & a.mi & & & -17.6 & $1 \leq 0$ & 0.0 & Ane $\mathrm{R}^{\mathrm{N}}$ \\
\hline 1000 & $100 \%$ & & & & का & 000 & $A=R=$ \\
\hline 2.125 & 197 & & & -7.2 & ni & $=10$ & due $p F$ \\
\hline 1234 & 1.191 & & & 35 & 192 & 0.0 & Ane Re \\
\hline 1.662 & $1 \mathrm{has}$ & & & 130 & 234 & a.15 & $\mu \in P=$ \\
\hline $1 m 9$ & 1901 & & & & ${ }_{607}$ & 000 & Aus iv \\
\hline 1007 & 0.977 & & & A. 1 & 201 & 0.00 & ANe PE: \\
\hline 9,163 & $1,9 \times 0$ & & & -79 & 200 & 0.00 & Wue $P$ Fe \\
\hline 5.131 & 1932 & & & -14 & 205 & tico & A.n Pr \\
\hline 1277 & 1.198 & & & \&1 & as & $a c 0$ & Nover \\
\hline 1000 & 2.090 & & & & 114 & 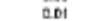 & ANe PE \\
\hline 1054 & 1at? & & & -1.9 & 270 & ant & ANo Fe \\
\hline 1297 & 1.118 & & & 11.0 & 212 & at & Awe RV \\
\hline 1,182 & 20.92 & & & 1.4 & 211 & 2.01 & whe Ror \\
\hline 1000 & 1,930 & & & & 84 & 0.01 & A.n VUt \\
\hline 1,520 & 1.37 & & & 0.5 & isd & 0.01 & Ano par \\
\hline 1371 & 1240 & & & 16 & se & 0.00 & wherer \\
\hline $125 n$ & 1221 & & & .23 & 170 & 0.01 & ANo R R \\
\hline 2.7os & 2,35 & & & 4.7 & 90 & 0.01 & $\operatorname{Mw} \mathrm{R}_{\mathrm{N}} \mathrm{F}$ \\
\hline 2.742 & 2.908 & & & 73 & 178 & 0.02 & 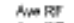 \\
\hline צמנג & & & & a.a & 170 & 0.01 & Awe RfF \\
\hline
\end{tabular}




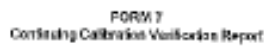

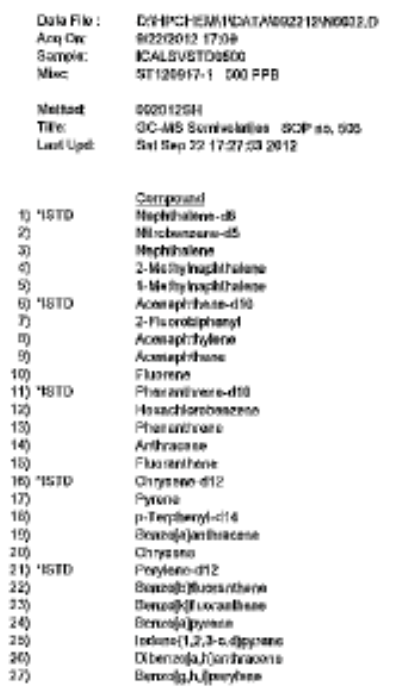

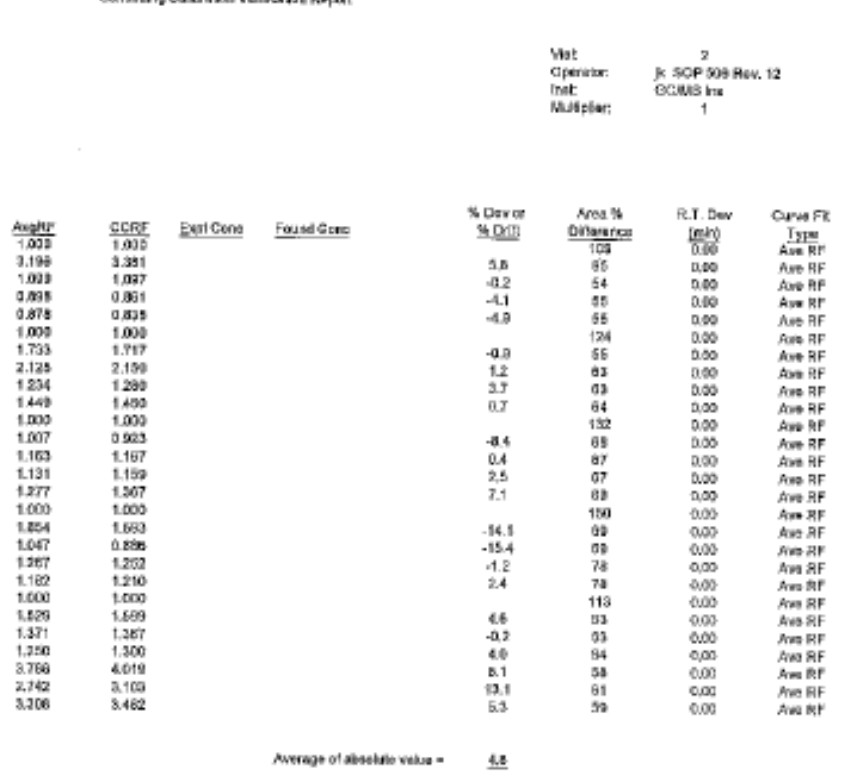

$\frac{84}{9-24-14}$

38 of 47 


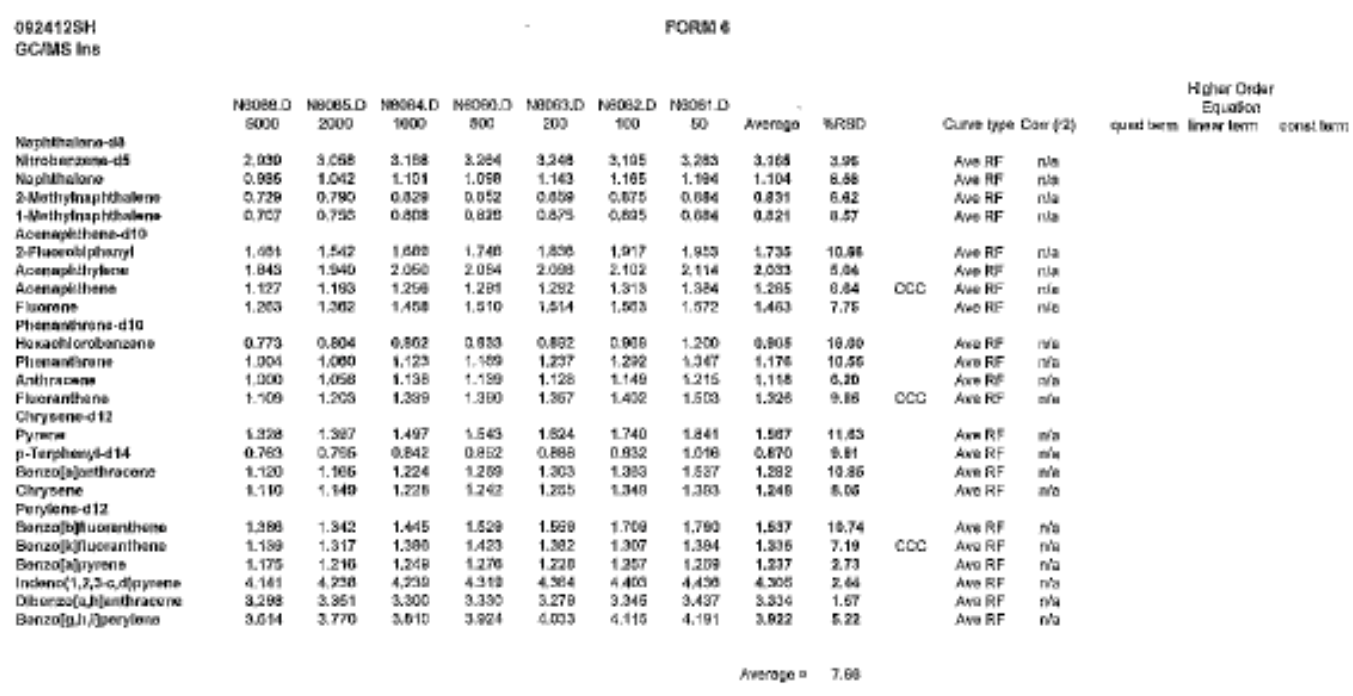




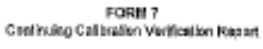

\begin{tabular}{|c|c|}
\hline 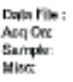 & 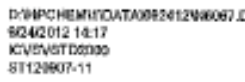 \\
\hline 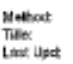 & 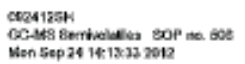 \\
\hline 1810 & 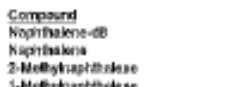 \\
\hline 1950 & $\begin{array}{l}\text { T-HeEshiaptededese } \\
\text { Monnopithnoed do }\end{array}$ \\
\hline$\infty$, & 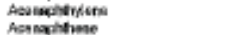 \\
\hline & $\begin{array}{l}\text { Fisereme } \\
\text { Phenvenengerdse }\end{array}$ \\
\hline & 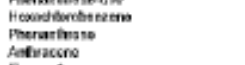 \\
\hline $\operatorname{coc}$ & nexarthese \\
\hline ISTD & 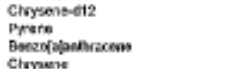 \\
\hline 18TD & 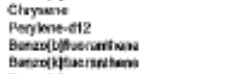 \\
\hline $\operatorname{coc}$ & 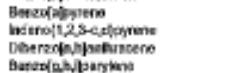 \\
\hline
\end{tabular}

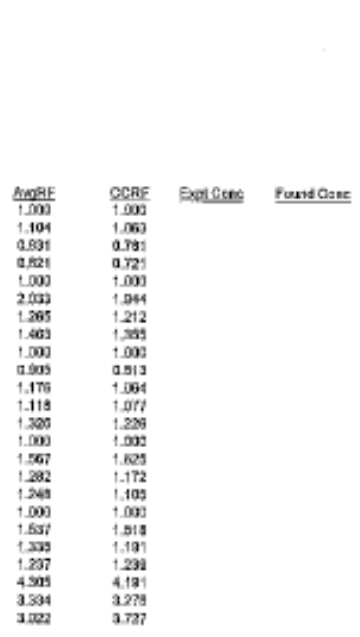

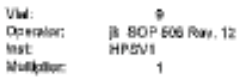

Averege ef atadista val ie -

\begin{tabular}{|c|c|c|c|}
\hline$\leqslant$ Daver & Areo $\mathrm{s}$ & R.T. Cas & curvon: \\
\hline & onverseces & $1 \tan x$ & Thet \\
\hline IY. & wo & 800 & $\sin 28 x$ \\
\hline \multirow{3}{*}{-12.2} & 160 & 0.00 & $\operatorname{lu}_{2} 8 F$ \\
\hline & 160 & 6.60 & $\sin 8$ \\
\hline & 102 & 0.00 & Aw $P F$ \\
\hline AS & 100 & 80 & Sine ist \\
\hline \multirow{3}{*}{$-3,4$} & 19 & 0.90 & $\operatorname{lng} P F$ \\
\hline & 160 & ave & the pro \\
\hline & 102 & 0.00 & Aus $R F$ \\
\hline-16.2 & 10 & 290 & Net RF \\
\hline$=2.5$ & 184 & a.st & Awe $\mathrm{RP}$ \\
\hline-27 & 194 & .0. & $\tan R F$ \\
\hline \multirow[t]{2}{*}{-7.8} & 187 & at: 10 & hose or \\
\hline & 100 & 0.00 & Aos RF \\
\hline 37 & 210 & 60 & Aase int \\
\hline 86 & 180 & Dor & $A \rightarrow R F$ \\
\hline \multirow[t]{2}{*}{ - $11, a$} & 100 & a.se & Axtef \\
\hline & $\%$ & 0.91 & Aop RF \\
\hline-1.8 & 200 & D.PI & AaP RF \\
\hline-16.8 & $1 \mathrm{~m}$ & a.p1 & Aou $\mathrm{AF}$ \\
\hline 0.2 & 186 & 0.01 & $\mathrm{~A}_{n \rightarrow \mathrm{RF}}$ \\
\hline-25 & 190 & 0.60 & $\operatorname{sos} \mathrm{AF}$ \\
\hline-1.7 & 190 & 0,00 & $A \cap R F$ \\
\hline-50 & 190 & a.to & boos RF \\
\hline
\end{tabular}

$\int_{2-25}^{94}$

40 of 47 
8B

Semi-Volatile Internal Standard Area Summary

Lab Name: ALS Environmental - FC

Date Analyzed: $\quad 9 / 22 / 2012$

Work Order Number: 1209339

Time Analyzed:

17:08

Client Name: Battelle Energy Alliance

ClientProject ID: BEA031231 MFC Biodiesel Tank Remova ,TOS-A1175 ReV2

Reported on: Tuesday. September 25, 2012

Instrument ID: HPSV1

Lab File ID: $\quad$ N8032

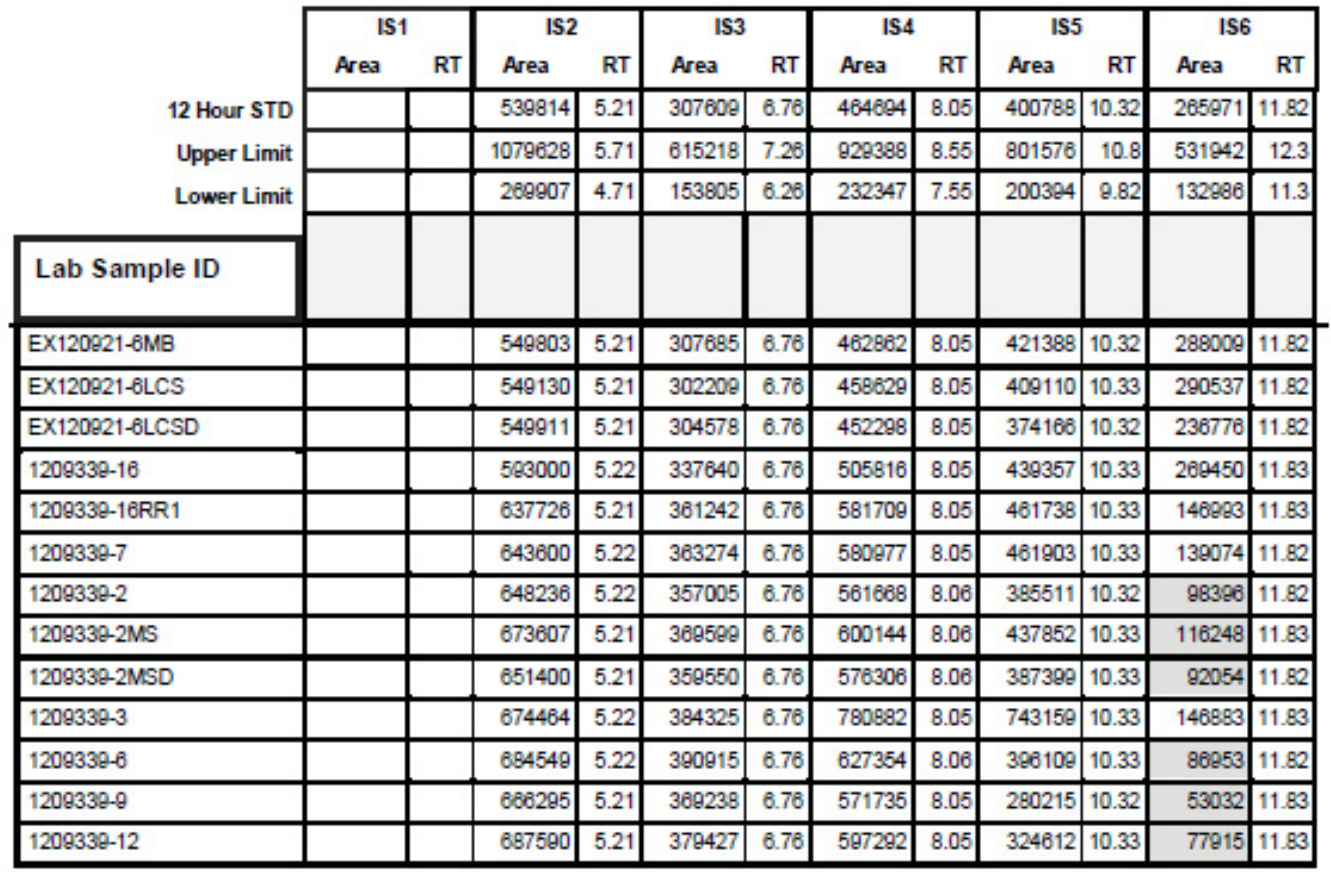




\section{B}

Semi-Volatile Internal Standard Area Summary

Lab Name: ALS Environmental - FC

Date Analyzed:

$9 / 24 / 2012$

Work Order Number: 1208338

Time Analyzed:

11:18

Client Name: Battelle Energy Alliance

ClientProject ID: BEA031231 MFC Biodiesel Tank Removal ,TOS-A1175 ReV2

Reported on: Tuesday. September 25, 2012

Instrument ID: HPSV1

Lab File ID: $\quad$ N6060

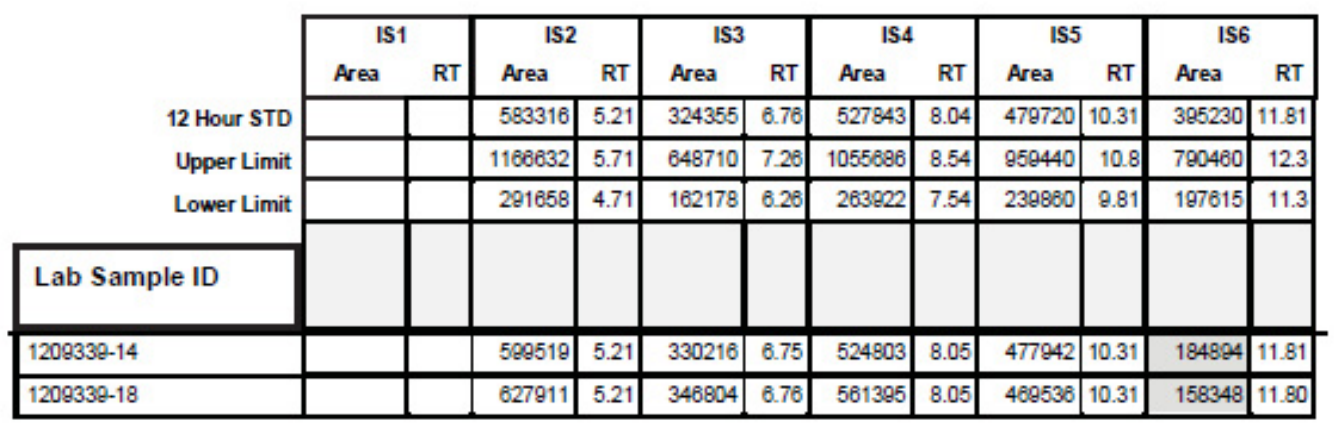

Shaded values exceed established area count limits.

LMS Verzion: 6.513

Upper Limit $=+100$ percent of internal standard area.

Lower Limit $=-50$ percent of internal standard area.

42 of 47 


\section{A}

\section{Supporting Raw Data}

43 of 47 

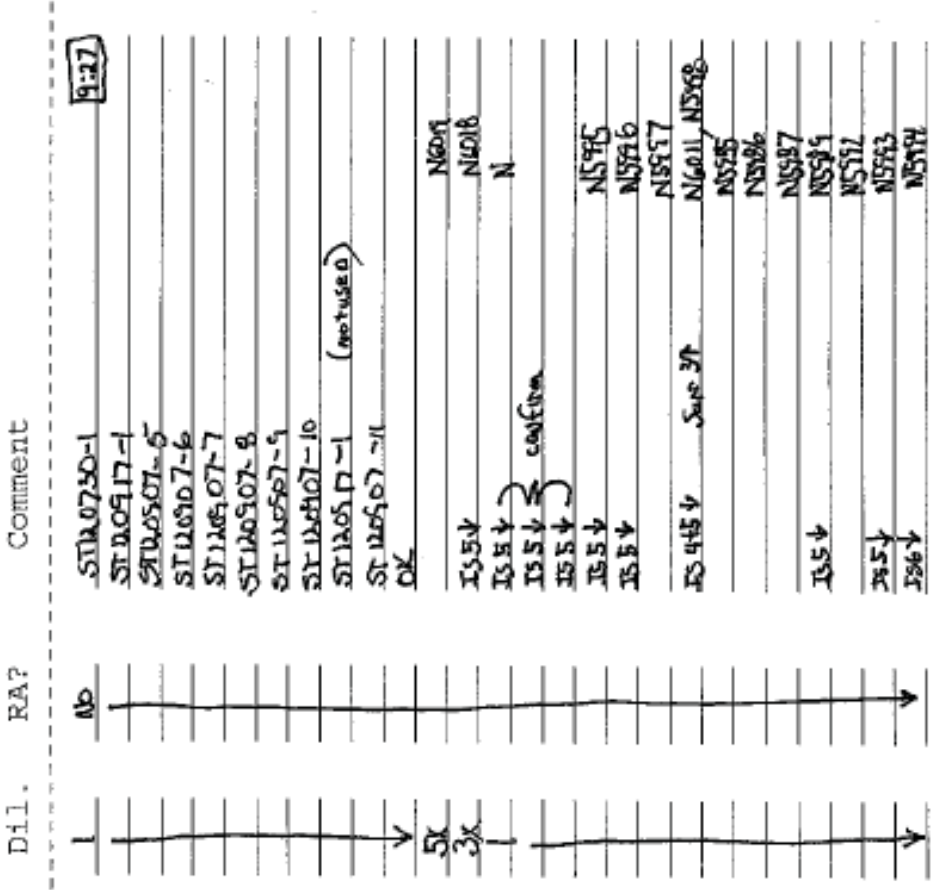

U

品o

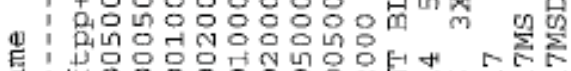

"

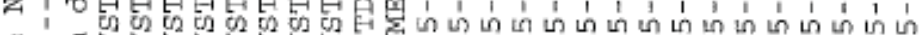

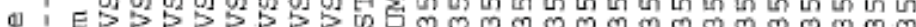
, 1 on 0 on Q 1 ,

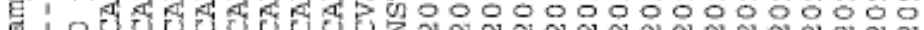
का

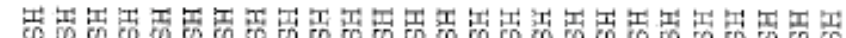
व ( म

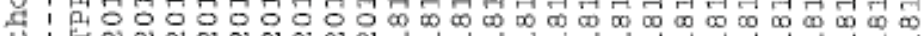
a ₹ 100000000000000000000000000 (4) !

i

म 出

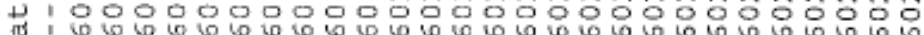

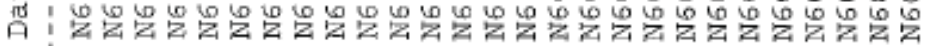
1 ल ro , ल m मी 


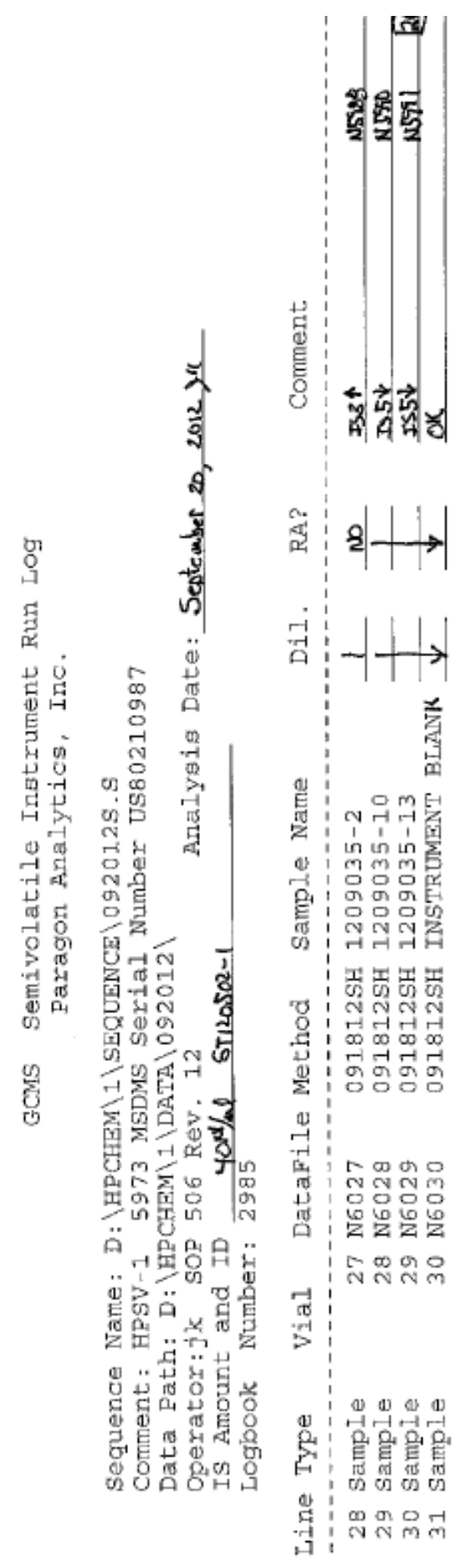

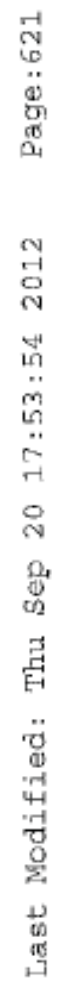

45 of 47 


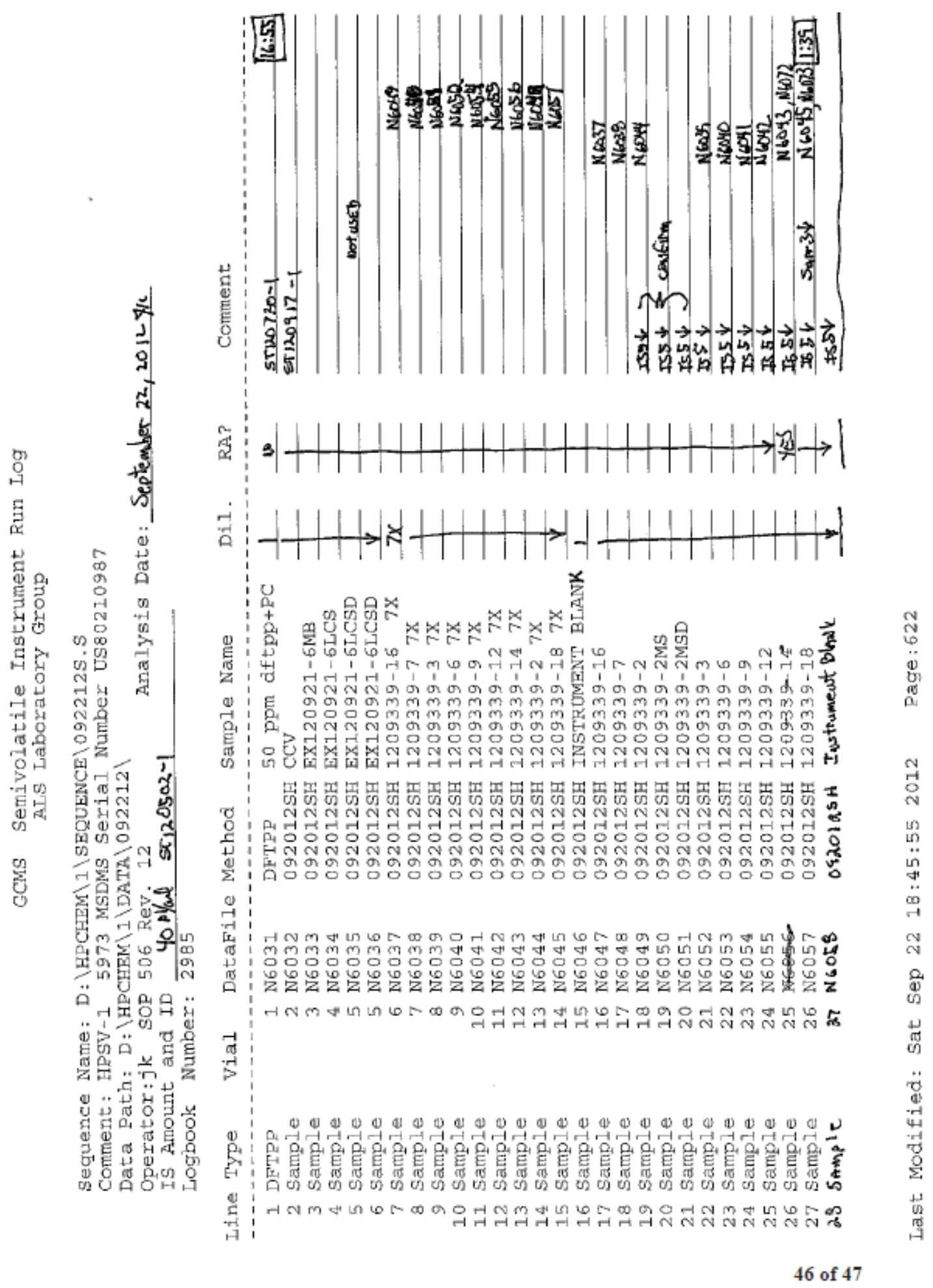



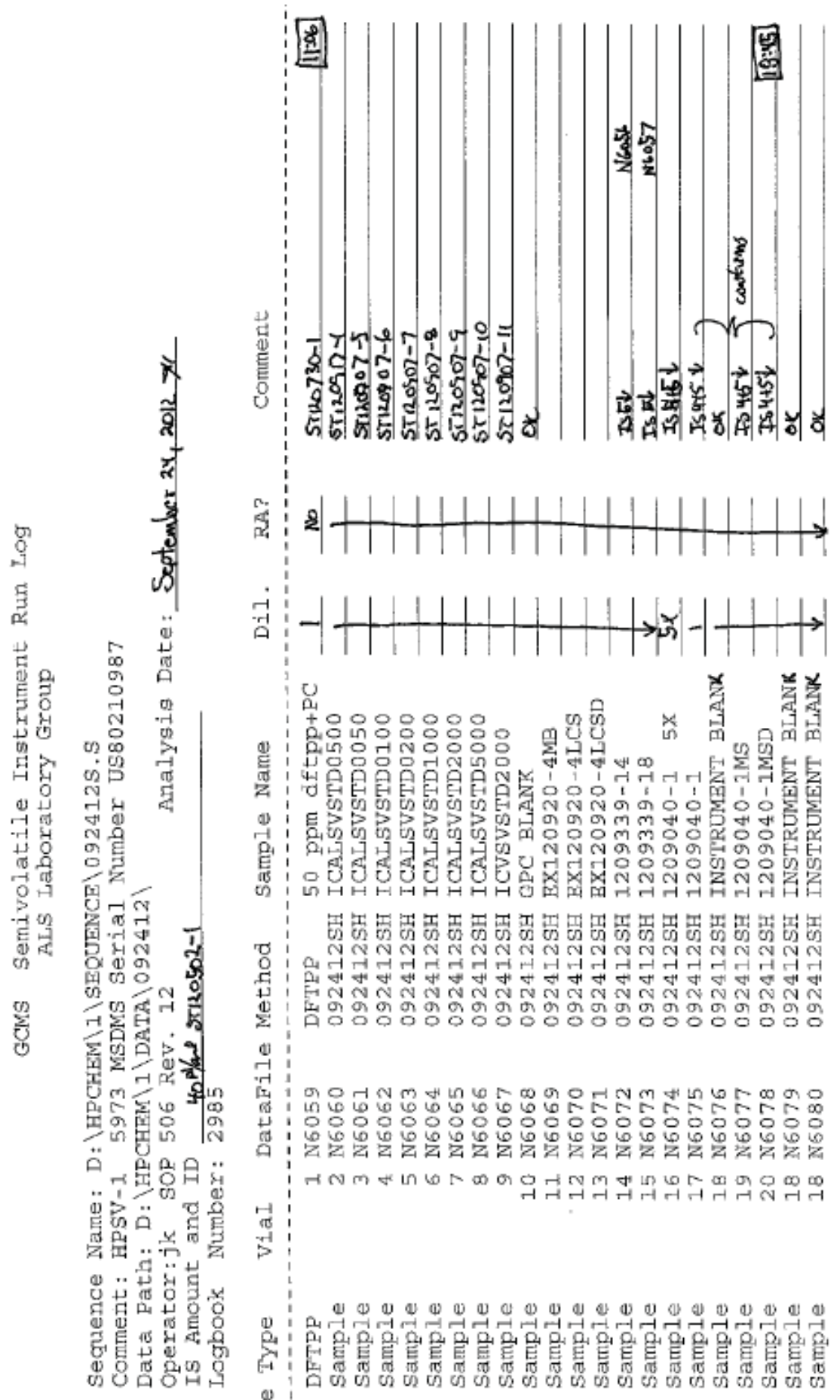

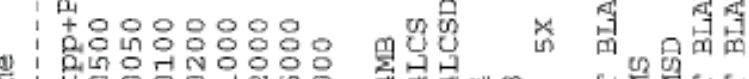

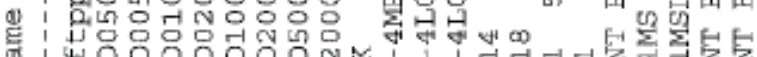

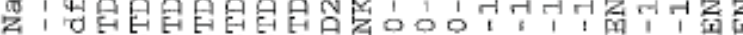

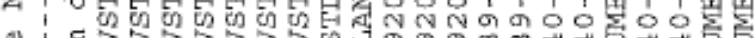

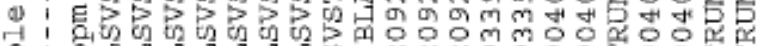
م 1 \%

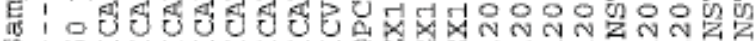

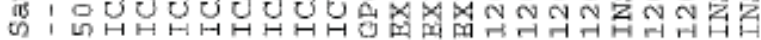

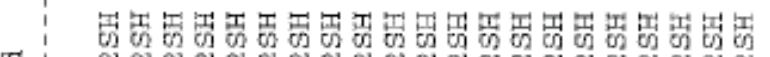
ग

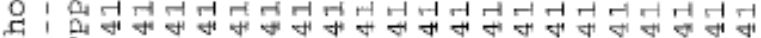

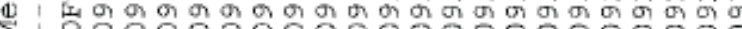
i:

II 90 - $N$ m th

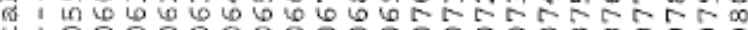

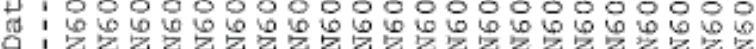

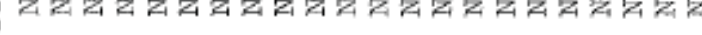
नल

(⿻)

3

(1)

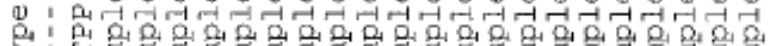

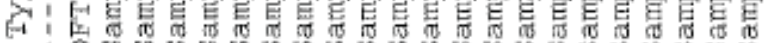

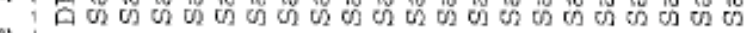

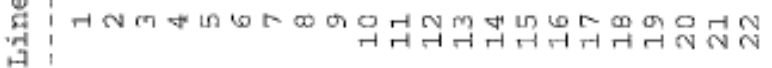




\section{Appendix G \\ Notification of Underground Storage Tank Soil Samples \#2 (CCN 228585)}

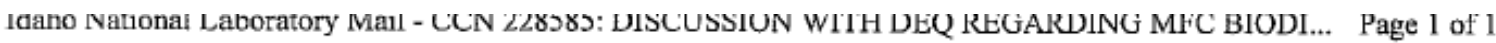

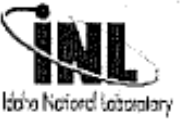

Nisson, Kerry L <kerry.nisson@inl.gov>

\section{CCN 228585: DISCUSSION WITH DEQ REGARDING MFC BIODIESEL TANK SAMPLES AND COMPLETION OF CORRECTIVE ACTIONS FOR OUTSTANDING NOTICE OF VIOLATIONS \\ 1 message}

Nisson, Kerry L <kerry,nisson@inl.gov>

Thu, Sep 27, 2012 at 7:56 AM

To: BEA CORRESPONDENCE CONTROL ServiceID <beacc@inl.gov>, ENVIRONMENTAL CORRESPONDENCE ServicelD <envaff@inl.gov>. Timothy L Carlson <Timothy.Carison@inl.gov>, James F Graham < James.Graham@inl.gov>, Timothy A

Miller <Timothy.Miller@inl.gov>, Bradley K Griffith <bradley.griffith@inl.gov>, "Jason R. Sturm" <sturmjr@id.doe.gov>, Scott L Lyman <Scott.Lyman@inl.gov>, Mark R Cole <mark.cole@inl.gov>, Reva A Nickelson <reva,nickelson@inl.gov>, Carlo D Melbihess <carlo.melbihess@inl.gov>

On September 26, 2012 at 15:10, a call was placed to Steve Heaton (DEQ) by Jason Sturm, Bradley Griffith, and I. The purpose of the call was to discuss analysis received for the second set of samples taken from the removal of the Materials and Fuels Complex (MFC) biodiesel tank and the completion of corrective actions for outstanding Notice of Violations (NOV's) associated with INL underground storage tanks.

Steve was informed that the second set of samples identified levels of Benzo(a)pyrene in nine of the ten samples higher than the screening level in IDAPA 58.01.24.800, table 2 - Residential Use Screening Levels.

Steve stated that we should use the commercial screening levels or perform a risk evaluation. Brad and I were unaware that there are commercial screening levels. Steve could not readily locate the commercial screening levels and stated that he would try to find them and would contact us.

Regarding the INL NOV's, Steve stated that he was aware that the INL had completed the corrective actions by the commitment date and would issue a letter of completion once the closure report was completed for the MFC biodiesel tank. Steve is aware that the corrective action (cathodic protection) is complete for the MFC biodiesel tank and the closure report will not be completed and sent to DEQ by the corrective action commitment date.

Kerry L. Nisson

Nuclear Operations Environmental Support - UST TPOC

Office (208) 533-7102

Cell (208) 569-4721

email: kerry.nisson@inl.gov

Mail Stop 6134 


\section{Appendix $\mathrm{H}$ \\ Discussion and Verbal Approval Regarding the Use of EPA Region 9 Industrial Screening Levels (CCN 228607)}

Idaho National Laboratory Mail - CCN 228607: DISCUSSION WITH DEQ REGARDING THE USE O... Page 1 of 1

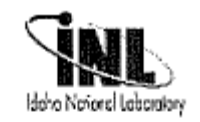

Nisson, Kerry L <kerry.nisson@inl.gov>

CCN 228607: DISCUSSION WITH DEQ REGARDING THE USE OF EPA REGION 9 INDUSTRIAL SCREENING LEVEL GUIDANCE.

1 message

Nisson, Kerry L <kerry.nisson@inl.gov> Thu, Sep 27, 2012 at 1:53 P
To: BEA CORRESPONDENCE CONTROL ServicelD <beacc@inl.gov>, ENVIRONMENTAL CORRESPONDENCE ServicelD

Thu, Sep 27, 2012 at 1:53 PM <envaff@inl.gov>, Timothy 1 Carlson <Timothy.Carlson@inl.gov>, James F Graham <James,Graham@inl.gov>, Timothy A Miller <Timothy.Miller@inl.gov>, Bradley K Griffith <bradley.griffith@inl.gov>, "Jason R. Sturm" <sturmjr@id.doe.gov>, Scott L Lyman <Scott.Lyman@inl,gov>, Mark R Cole <mark.cole@inl.gov>, Reva A Nickelson <reva.nickelson@inl.gov>, Carlo D Melbihess <carlo.melbihess@inl.gov>, Lynne M Coe-Leavitt <Lynne.Coe-Leavitt@inl.gov>, Tim A Solle<Tim.Solle@inl.gov>

On September 26, 2012 at 11:00, a call was placed to Steve Heaton (DEQ) by Jason Sturm, Gary McManus, and I. The purpose of the call was to discuss the use of industrial screening levels found in the Environmental Protection Agency (EPA) guidelines for Region 9.

Background: After talking with Steve Heaton, DEQ yesterday, there was a search for guidance for commercial screening levels in the Idaho Risk Evaluation Manual for Petroleum Releases as suggested by Steve. No guidance could be found in the manual. An internet search revealed EPA guidelines for industrial screening levels for Region 9, but could not find any guidance for Region 10. Gary McManus utilized a download spreadsheet provided in the EPA website and processed the sampling analysis Region 9 using ingestion levels (most conservative). The samples levels were lower that the industrial screening levels.

The call was placed to Steve Heaton (DEQ) to explain the actions taken above. Steve wanted to know if we had found any guidance for commercial screening levels in the Idaho Risk Evaluation Manual for Petroleum Releases. I told him that we were unable to identify any guidance in the manual. Steve responded that he was acceptable to using the EPA Region 9 guidance and gave verbal authorization to continue. Steve requested that the EPA Region 9 information be included in the tank closure plan. I communicated to Steve that I was prepared to e-mail him the sampling analysis and the spreadsheet that we utilized to review. Steve replied that he would, so the information was sent. Steve responded that he had received the e-mail. I requested that he contact us if he encountered any issues and that we would be proceeding with the construction project.

Kerry L. Nisson

Nuclear Operations Environmental Support - UST TPOC

Office (208) 533-7102

Cell (208) 569-4721

email: kerry.nisson@inl.gov

Mail Stop 6134 


\section{Appendix I \\ Spreadsheet Comparison of EPA Region 9 Industrial Screening Levels vs. IDAPA Residential Screening Levels}

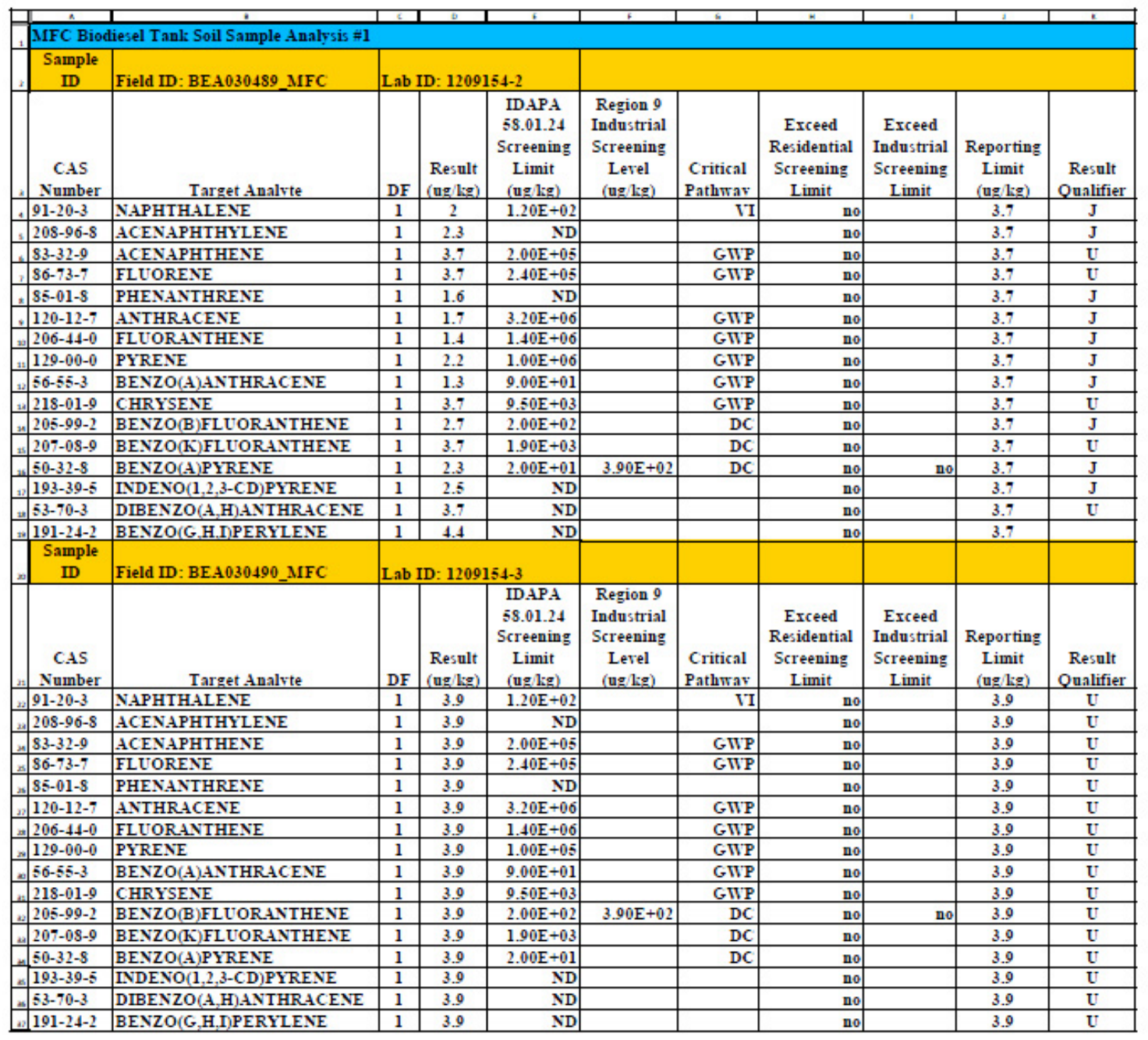




\begin{tabular}{|c|c|c|c|c|c|c|c|c|c|c|c|}
\hline & $n$ & $\therefore$ & 1 & b & 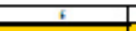 & $F$ & $\overline{6}$ & 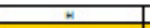 & 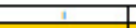 & I & $\bar{E}$ \\
\hline$=$ & $\begin{array}{c}\text { Sample } \\
\text { ID }\end{array}$ & Field ID: BEA030493_MFC & \multicolumn{3}{|c|}{ Lab ID: $1209154-6$} & & & & & & \\
\hline$\infty$ & $\begin{array}{c}\text { CAS } \\
\text { number }\end{array}$ & Target Analvte & DF & $\begin{array}{c}\text { Result } \\
(\mathrm{ug} / \mathrm{kg})\end{array}$ & $\begin{array}{c}\text { IDAPA } \\
58.01 .24 \\
\text { Screening } \\
\text { Limit } \\
(\mathrm{ug} / \mathrm{kg})\end{array}$ & $\begin{array}{c}\text { Region } 9 \\
\text { Industrial } \\
\text { Screening } \\
\text { Level } \\
\text { (ug/kg) }\end{array}$ & $\begin{array}{l}\text { Critical } \\
\text { Pathwav }\end{array}$ & $\begin{array}{c}\text { Exceed } \\
\text { Residential } \\
\text { Screening } \\
\text { Limit }\end{array}$ & $\begin{array}{c}\text { Exceed } \\
\text { Industrial } \\
\text { Screening } \\
\text { Limit } \\
\end{array}$ & $\begin{array}{c}\text { Reporting } \\
\text { Limit } \\
\text { (ug/ } / \mathrm{kg})\end{array}$ & $\begin{array}{c}\text { Result } \\
\text { Qualifier }\end{array}$ \\
\hline+ & $91-20-3$ & NAPHTH_ALENE & 1 & 3.6 & $1.20 \mathrm{E}+02$ & & VI & no & & 3.6 & $\mathrm{U}$ \\
\hline 4 & $208-96-8$ & ACENAPHTHYLENE & 1 & 3.6 & $\mathrm{ND}$ & & & no & & 3.6 & $\mathrm{U}$ \\
\hline 42. & $83-32-9$ & ACENAPHTHENE & 1 & 3.6 & $2.00 \mathrm{E}+05$ & & GWP & no & & 3.6 & $\mathrm{U}$ \\
\hline 4 & $86-73-7$ & FLUORENE & 1 & 1.3 & $2.40 \mathrm{E}+05$ & & GWP & no & & 3.6 & $\mathrm{~J}$ \\
\hline+4 & $85-01-8$ & PHENANTHRENE & 1 & 31 & $\mathrm{ND}$ & & & no & & 3.6 & \\
\hline s. & $120-12-7$ & ANTHRACENE & 1 & 10 & $3.20 \mathrm{E}+06$ & & GWP & no & & 3.6 & \\
\hline 4 & $206-44-0$ & FLUORANTHENE & 1 & 280 & $1.40 \mathrm{E}+06$ & & GWP & no & & 3.6 & E \\
\hline 4 & 129-00-0 & PYRENE & 1 & 170 & $1.00 \mathrm{E}+05$ & & GWP & no & & 3.6 & \\
\hline 4 & $56-55-3$ & BENZO $(A) A N T H R A C E N E$ & 1 & 180 & $9.00 \mathrm{E}+01$ & $3.90 \mathrm{E}+03$ & GWP & YES & No & 3.6 & $\mathbf{E}$ \\
\hline 4 & $218-01-9$ & CHRYSENE & 1 & 190 & $9.50 \mathrm{E}+03$ & & GTP & no & & 3.6 & $\mathbf{E}$ \\
\hline 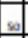 & $205-99-2$ & BENZO(B)FLUORANTHENE & 1 & 330 & $2.00 \mathrm{E}+02$ & $3.90 \mathrm{E}+03$ & $\mathrm{DC}$ & YES & No & 3.6 & E \\
\hline$s$ & $207-08-9$ & BENZO(K)FLUORANTHENE & 1 & 130 & $1.90 \mathrm{E}+03$ & & $\mathrm{DC}$ & no & & 3.6 & \\
\hline $\mathrm{sis}$ & $50-32-8$ & BENZO(A)PYRENE & 1 & 240 & $2.00 \mathrm{E}+01$ & $3.90 \mathrm{E}+02$ & $\mathrm{DC}$ & YES & $\mathrm{NO}$ & 3.6 & $\mathbf{E}$ \\
\hline $\mathrm{s}$ & $193-39-5$ & INDENO(1,2,3-CD)PYRENE & 1 & 120 & $\mathrm{ND}$ & & & no & & 3.6 & \\
\hline si & $53-70-3$ & DIBENZO $(A, H)$ ANTHRACENE & 1 & 35 & ND & & & no & & 3.6 & \\
\hline st & $191-24-2$ & BENZO(G,H.I)PERYLENE & 1 & 110 & ND & & & not & & 3.6 & \\
\hline 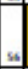 & $\begin{array}{c}\text { Sample } \\
\text { ID }\end{array}$ & Field ID: BEA030494_MFC & Lab I & D: 12091 & $54-7$ & & & & & & \\
\hline 5 & $\begin{array}{c}\text { CAS } \\
\text { Number }\end{array}$ & Iarget Analvte & DF & $\begin{array}{r}\text { Result } \\
(\mathrm{ug} / \mathrm{kg})\end{array}$ & $\begin{array}{c}\text { IDAPA } \\
58.01 .24 \\
\text { Screening } \\
\text { Limit } \\
\text { (ug/kg) }\end{array}$ & $\begin{array}{c}\text { Region } 9 \\
\text { Industrial } \\
\text { Screening } \\
\text { Level } \\
\text { (ug/kg) }\end{array}$ & $\begin{array}{c}\text { Critical } \\
\text { Pathway }\end{array}$ & $\begin{array}{c}\text { Exceed } \\
\text { Residential } \\
\text { Screening } \\
\text { Limit } \\
\end{array}$ & $\begin{array}{c}\text { Exceed } \\
\text { Industrial } \\
\text { Screening } \\
\text { Limit } \\
\end{array}$ & $\begin{array}{c}\text { Reporting } \\
\text { Limit } \\
\text { (ug/kg) } \\
\end{array}$ & $\begin{array}{c}\text { Result } \\
\text { Qualifier }\end{array}$ \\
\hline st & $91-20-3$ & NAPHTHALENE & 1 & 3.7 & $1.20 \mathrm{E}+02$ & & VI & no & & 3.7 & $\mathrm{U}$ \\
\hline 5 & $208-96-8$ & ACENAPHTHYLENE & 1 & 3.7 & $\mathrm{ND}$ & & & no & & 3.7 & $\mathrm{U}$ \\
\hline 然 & $83-32-9$ & ACENAPHTHENE & 1 & 3.7 & $2.00 \mathrm{E}+05$ & & GIVP & no & & 3.7 & $\mathrm{U}$ \\
\hline 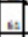 & $86-73-7$ & FLUORENE & 1 & 3.7 & $2.40 \mathrm{E}+05$ & & GIVP & no & & 3.7 & $\mathrm{U}$ \\
\hline 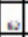 & $85-01-8$ & PHENANTHRENE & 1 & 3.7 & $\mathrm{ND}$ & & & no & & 3.7 & $\mathrm{~J}$ \\
\hline$\Delta$ & $120-12-7$ & ANTHRACENE & 1 & 3.7 & $3.20 \mathrm{E}+06$ & & GIVP & no & & 3.7 & $\mathrm{U}$ \\
\hline का & $206-44-0$ & FLUORANTHENE & 1 & 21 & $1.40 \mathrm{E}+06$ & & GIVP & no & & 3.7 & \\
\hline$s$ & $129-00-0$ & PYRENE & 1 & 16 & $1.00 \mathrm{E}+05$ & & GWP & no & & 3.7 & \\
\hline 4 & $56-55-3$ & BENZO(A)ANTHRACENE & 1 & 15 & $9.00 \mathrm{E}+01$ & & GWP & no & & 3.7 & \\
\hline 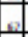 & $218-01-9$ & CHRYSENE & 1 & 18 & $9.50 \mathrm{E}+03$ & & GIVP & no & & 3.7 & \\
\hline 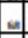 & $205-99-2$ & BENZO(B)FLUORANTHENE & 1 & 24 & $2.00 \mathrm{E}+02$ & & $\mathrm{DC}$ & no & & 3.7 & \\
\hline$=$ & $207-08-9$ & BENZO(K)FLUORANTHENE & 1 & 12 & $1.90 \mathrm{E}+03$ & & $\mathrm{DC}$ & no & & 3.7 & \\
\hline$a$ & $50-32-8$ & BENZO(A)PYRENE & 1 & 19 & $2.00 \mathrm{E}+01$ & $3.90 \mathrm{E}+02$ & $\mathrm{DC}$ & no & no & 3.7 & \\
\hline 3 & $193-39-5$ & INDENO(1,2,3-CD)PYRENE & 1 & 14 & $\mathrm{ND}$ & & & no & & 3.7 & \\
\hline 2 & $53-70-3$ & DIBENZO $(A, H)$ ANTHRACENE & 1 & 4.1 & $\mathrm{ND}$ & & & no & & 3.7 & \\
\hline 2 & $191-24-2$ & BENZO(G,H,I)PERYLENE & 1 & 14 & $\mathrm{ND}$ & & & no & & 3.7 & \\
\hline
\end{tabular}




\begin{tabular}{|c|c|c|c|c|c|c|c|c|c|c|c|}
\hline & $n$ & $\therefore$ & L & b & 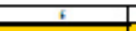 & $F$ & $\bar{G}$ & n & 1 & I & $\bar{E}$ \\
\hline - & $\begin{array}{c}\text { Sample } \\
\text { ID }\end{array}$ & Field ID: BEA 030497_MFC & \multicolumn{3}{|c|}{ Lab ID: $1209154-10$} & & & & & & \\
\hline $\mathrm{x}$ & $\begin{array}{c}\text { CAS } \\
\text { number }\end{array}$ & Target Analvte & $\mathrm{DF}$ & $\begin{array}{c}\text { Result } \\
(\mathrm{ug} / \mathrm{kg})\end{array}$ & $\begin{array}{c}\text { IDAPA } \\
58.01 .24 \\
\text { Screening } \\
\text { Limit } \\
(\mathrm{ug} / \mathrm{kg})\end{array}$ & $\begin{array}{c}\text { Region } 9 \\
\text { Industrial } \\
\text { Screening } \\
\text { Level } \\
\text { (ug/kg) }\end{array}$ & $\begin{array}{l}\text { Critical } \\
\text { Pathwav }\end{array}$ & $\begin{array}{c}\text { Exceed } \\
\text { Residential } \\
\text { Screening } \\
\text { Limit }\end{array}$ & $\begin{array}{c}\text { Exceed } \\
\text { Industrial } \\
\text { Screening } \\
\text { Limit }\end{array}$ & $\begin{array}{c}\text { Reporting } \\
\text { Limit } \\
\text { (ug/ } / \mathrm{kg})\end{array}$ & $\begin{array}{c}\text { Result } \\
\text { Qualifier }\end{array}$ \\
\hline 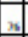 & $91-20-3$ & NAPHTH_ALENE & 1 & 3.7 & $1.20 \mathrm{E}+02$ & & VI & no & & 3.7 & $\mathrm{U}$ \\
\hline 亦 & $208-96-8$ & ACENAPHTHYLENE & 1 & 3.7 & $\mathrm{ND}$ & & & no & & 3.7 & $\mathrm{U}$ \\
\hline 至 & $83-32-9$ & ACENAPHTHENE & 1 & 3.7 & $2.00 \mathrm{E}+05$ & & GWP & no & & 3.7 & $\mathrm{U}$ \\
\hline$n$ & $86-73-7$ & FLUORENE & 1 & 1.7 & $2.40 \mathrm{E}+05$ & & GWP & no & & 3.7 & $\mathrm{~J}$ \\
\hline 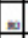 & $85-01-8$ & PHENANTHRENE & 1 & 10 & $\mathrm{ND}$ & & & no & & 3.7 & \\
\hline 至 & $120-12-7$ & ANTHRACENE & 1 & 3.1 & $3.20 \mathrm{E}+06$ & & GWP & no & & 3.7 & $\mathrm{~J}$ \\
\hline 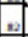 & $206-44-0$ & FLUORANTHENE & 1 & 40 & $1.40 \mathrm{E}+06$ & & GWP & no & & 3.7 & \\
\hline 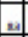 & 129-00-0 & PYRENE & 1 & 30 & $1.00 \mathrm{E}+05$ & & GWP & no & & 3.7 & \\
\hline st & $56-55-3$ & BENZO(A)ANTHRACENE & 1 & 30 & $9.00 \mathrm{E}+01$ & & GWP & no & & 3.7 & \\
\hline 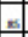 & $218-01-9$ & CHRYSENE & 1 & 35 & $9.50 \mathrm{E}+03$ & & GIVP & no & & 3.7 & \\
\hline 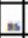 & $205-99-2$ & BENZO(B)FLUORANTHENE & 1 & 56 & $2.00 \mathrm{E}+02$ & & $\mathrm{DC}$ & no & & 3.7 & \\
\hline 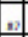 & $207-08-9$ & BENZO(K)FLUORANTHENE & 1 & 22 & $1.90 \mathrm{E}+03$ & & $\mathrm{DC}$ & no & & 3.7 & \\
\hline$=$ & $50-32-8$ & BENZO(A)PYRENE & 1 & 40 & $2.00 \mathrm{E}+01$ & $3.90 \mathrm{E}+02$ & $\mathrm{DC}$ & YES & No & 3.7 & \\
\hline- & $193-39-5$ & INDENO(1,2,3-CD)PYRENE & 1 & 22 & $\mathrm{ND}$ & & & no & & 3.7 & \\
\hline$\infty$ & $53-70-3$ & DIBENZO $(A, H)$ ANTHRACENE & 1 & 6.4 & ND & & & no & & 3.7 & \\
\hline+ & $191-24-2$ & BENZO(G,H.I)PERYLENE & 1 & 21 & ND & & & no & & 3.7 & \\
\hline. & $\begin{array}{c}\text { Sample } \\
\text { ID }\end{array}$ & Field ID: BEA03049s_MFC & Lab I & D: 12091 & 54-11 & & & & & & \\
\hline+ & $\begin{array}{c}\text { CAS } \\
\text { Number }\end{array}$ & Iarget Analvte & DF & $\begin{array}{r}\text { Result } \\
(\mathrm{ug} / \mathrm{kg})\end{array}$ & $\begin{array}{c}\text { IDAPA } \\
58.01 .24 \\
\text { Screening } \\
\text { Limit } \\
(\mathrm{ug} / \mathrm{lg}) \\
\end{array}$ & $\begin{array}{c}\text { Region } 9 \\
\text { Industrial } \\
\text { Screening } \\
\text { Level } \\
\text { (ug/kg) }\end{array}$ & $\begin{array}{c}\text { Critical } \\
\text { Pathway }\end{array}$ & $\begin{array}{c}\text { Exceed } \\
\text { Residential } \\
\text { Screening } \\
\text { Limit } \\
\end{array}$ & $\begin{array}{c}\text { Exceed } \\
\text { Industrial } \\
\text { Screening } \\
\text { Limit } \\
\end{array}$ & $\begin{array}{c}\text { Reporting } \\
\text { Limit } \\
\text { (ug/kg) } \\
\end{array}$ & $\begin{array}{c}\text { Result } \\
\text { Qualifier }\end{array}$ \\
\hline$m$ & $91-20-3$ & NAPHTHALENE & 1 & 3.7 & $1.20 \mathrm{E}+02$ & & VI & no & & 3.7 & $\mathrm{U}$ \\
\hline$s$ & $208-96-8$ & ACENAPHTHYLENE & 1 & 3.7 & $\mathrm{ND}$ & & & no & & 3.7 & $\mathrm{U}$ \\
\hline 然 & $83-32-9$ & ACENAPHTHENE & 1 & 3.7 & $2.00 \mathrm{E}+05$ & & GIVP & no & & 3.7 & $\mathrm{U}$ \\
\hline. & $86-73-7$ & FLUORENE & 1 & 3.7 & $2.40 \mathrm{E}+05$ & & GIVP & no & & 3.7 & $\mathrm{U}$ \\
\hline$=$ & $85-01-8$ & PHENANTHRENE & 1 & 17 & $\mathrm{ND}$ & & & no & & 3.7 & \\
\hline 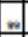 & $120-12-7$ & ANTHRACENE & 1 & 6.1 & $3.20 \mathrm{E}+06$ & & GIVP & no & & 3.7 & \\
\hline 果 & $206-44-0$ & FLUORANTHENE & 1 & 140 & $1.40 \mathrm{E}+06$ & & GTVP & no & & 3.7 & \\
\hline 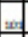 & $129-00-0$ & PYRENE & 1 & 85 & $1.00 \mathrm{E}+05$ & & GWP & no & & 3.7 & \\
\hline 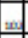 & $56-55-3$ & BENZO(A)ANTHRACENE & 1 & 94 & $9.00 \mathrm{E}+01$ & $3.90 \mathrm{E}+03$ & GWP & YES & No & 3.7 & \\
\hline 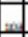 & $218-01-9$ & CHRYSENE & 1 & 96 & $9.50 \mathrm{E}+03$ & & GWP & no & & 3.7 & \\
\hline 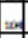 & $205-99-2$ & BENZO(B)FLUORANTHENE & 1 & 150 & $2.00 \mathrm{E}+02$ & & $\mathrm{DC}$ & no & & 3.7 & \\
\hline 再 & $207-08-9$ & BENZO(K)FLUORANTHENE & 1 & 57 & $1.90 \mathrm{E}+03$ & & $\mathrm{DC}$ & no & & 3.7 & \\
\hline 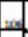 & $50-32-8$ & BENZO(A)PYRENE & 1 & 110 & $2.00 \mathrm{E}+01$ & $3.90 \mathrm{E}+02$ & $\mathrm{DC}$ & YES & $\mathrm{NO}$ & 3.7 & \\
\hline 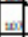 & $193-39-5$ & INDENO(1,2,3-CD)PYRENE & 1 & 66 & $\mathrm{ND}$ & & & no & & 3.7 & \\
\hline$\Rightarrow$ & $53-70-3$ & DIBENZO $(A, H)$ ANTHRACENE & 1 & 19 & $\mathrm{ND}$ & & & no & & 3.7 & \\
\hline 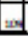 & $191-24-2$ & BENZO(G,H,I)PERYLENE & 1 & 63 & $\mathrm{ND}$ & & & no & & 3.7 & \\
\hline
\end{tabular}




\begin{tabular}{|c|c|c|c|c|c|c|c|c|c|c|c|}
\hline I & 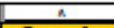 & 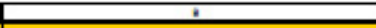 & 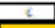 & b & 6 & I & G & 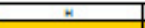 & $i$ & $i$ & E \\
\hline$=$ & $\begin{array}{l}\text { Sample } \\
\text { ID }\end{array}$ & Field ID: BEA030493_MFC & Lab & [D: 1209$]$ & 54-6RR1 & & & & & & \\
\hline s] & $\begin{array}{c}\text { CAS } \\
\text { Number }\end{array}$ & Target Analyte & DF & $\begin{array}{l}\text { Result } \\
(\mathrm{ug} / \mathrm{lg})\end{array}$ & $\begin{array}{c}\text { IDAPA } \\
58.01 .24 \\
\text { Screening } \\
\text { Limit } \\
(\mathrm{ug} / \mathrm{kg})\end{array}$ & $\begin{array}{c}\text { Region 9 } \\
\text { Industrial } \\
\text { Screening } \\
\text { Level } \\
(\mathrm{ug} / \mathrm{kg}) \\
\end{array}$ & $\begin{array}{c}\text { Critical } \\
\text { Pathway }\end{array}$ & $\begin{array}{c}\text { Exceed } \\
\text { Residential } \\
\text { Screening } \\
\text { Limit } \\
\end{array}$ & $\begin{array}{c}\text { Exceed } \\
\text { Industrial } \\
\text { Screening } \\
\text { Limit } \\
\end{array}$ & $\begin{array}{c}\text { Reporting } \\
\text { Limit } \\
(\mathrm{ug} / \mathrm{kg}) \\
\end{array}$ & $\begin{array}{c}\text { Result } \\
\text { Qualifier }\end{array}$ \\
\hline 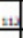 & 91-20-3 & NAPHITHAIENE. & 3 & 11 & $1.20 \mathrm{E}+02$ & & II & no & & 11 & II \\
\hline H. & $208-96-8$ & ACENAPHTHYLENE & 3 & 11 & $\mathrm{ND}$ & & & no & & 11 & U \\
\hline 4 & $83-32-9$ & ACENAPHTHENE & $\underline{3}$ & 11 & $2.00 \mathrm{E}+05$ & & GIVP & no & & 11 & U \\
\hline ts & $86-73-7$ & FLUORENE & 3 & 11 & $2.40 \mathrm{E}+05$ & & GIVP & no & & 11 & $\mathrm{U}$ \\
\hline 31 & $85-01-8$ & PHENANTHRFNE & $\underline{3}$ & $\underline{29}$ & ND & & & no & & 11 & \\
\hline if & $120-12-7$ & ANTHRACENE & $\underline{3}$ & 9.2 & $3.20 \mathrm{E}+06$ & & GTV & no & & 11 & $\mathrm{~J}$ \\
\hline & $206-44-0$ & FLUORANTHENE & $\underline{3}$ & $\underline{220}$ & $1.40 \mathrm{E}+06$ & & GTVP & no & & 11 & \\
\hline 프 & $129-00-0$ & PYRENE & 3 & 120 & $1.00 \mathrm{E}+05$ & & GIVP & no & & 11 & \\
\hline 년 & $56-55-3$ & BENZO(A)ANTHRACENE & 3 & 130 & $9.00 \mathrm{E}+01$ & $3.90 \mathrm{E}+03$ & GIVP & YES & No & 11 & \\
\hline tie & $218-01-9$ & CHRYSENE & $\underline{3}$ & $\underline{130}$ & $9.50 \mathrm{E}+03$ & & GTP & no & & 11 & \\
\hline 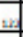 & $205-99-2$ & BENZO(B)ELUORANTHENE & 3 & 210 & $2.00 \mathrm{E}+02$ & $3.00 \mathrm{E}+0.3$ & $\mathrm{DC}$ & YES & No & 11. & \\
\hline is & $207-08-9$ & BENZO(K)FLUORANTHENE & $\underline{3}$ & 74 & $1.90 \mathrm{E}+03$ & & $\mathrm{DC}$ & no & & 11 & \\
\hline 政 & $50-32-8$ & BENZO(A)PYRENE & $\underline{3}$ & $\underline{150}$ & $2.00 \mathrm{E}+01$ & $3.90 \mathrm{E}+02$ & $\underline{\mathrm{DC}}$ & YES & $\mathrm{NO}$ & 11 & \\
\hline 角 & $193-39-5$ & INDENO(1,2,3-CD)PYRENE & 3 & 94 & ND & & & no & & 11 & \\
\hline 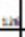 & $53-70-3$ & DIBENZO $(A, H) A N T H R A C E N E$ & $\underline{3}$ & $\underline{26}$ & ND & & & no & & 11 & \\
\hline 过 & $191-24-2$ & BENZO(G.H.I)PERYLENE & 3 & 89 & ND & & & no & & 11 & \\
\hline
\end{tabular}




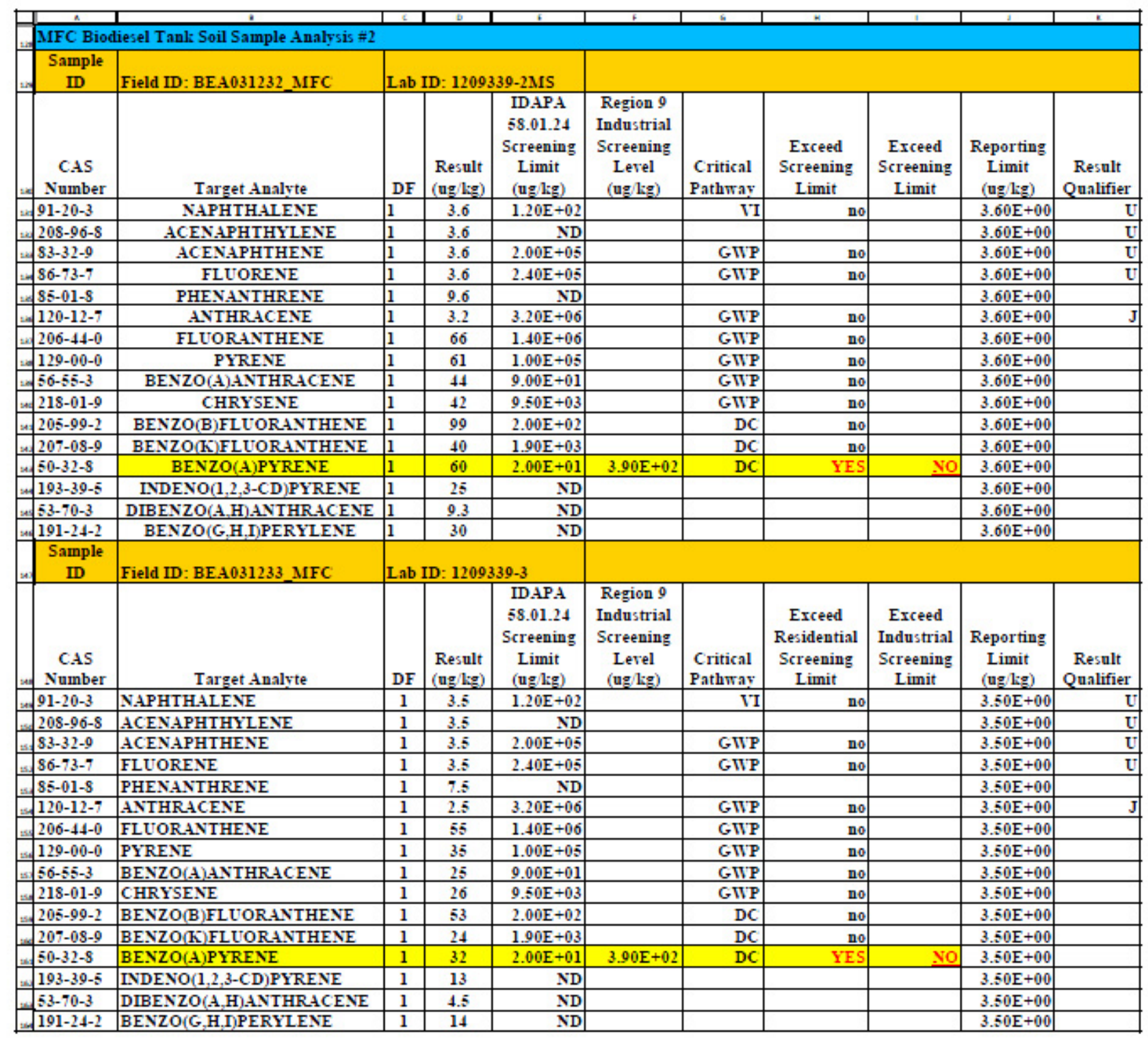




\begin{tabular}{|c|c|c|c|c|c|c|c|c|c|c|c|}
\hline \multirow{2}{*}{ - } & \multirow{2}{*}{\begin{tabular}{|c} 
Sample \\
ID
\end{tabular}} & i & \multicolumn{2}{|c|}{\begin{tabular}{|l|l|l|l|} 
& 0 \\
\end{tabular}} & \begin{tabular}{|l|l}
6 \\
\end{tabular} & & $\begin{array}{ll} \\
\end{array}$ & $\begin{array}{ll}\mathrm{n} \\
\mathrm{n}\end{array}$ & \multicolumn{2}{|r|}{ 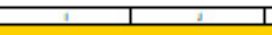 } & \multirow{2}{*}{ s } \\
\hline & & Field ID: BEA031236_MFC & \multicolumn{3}{|c|}{ Lab ID: $1209339-6$} & & & & & & \\
\hline$=$ & $\begin{array}{c}\text { CAS } \\
\text { Number }\end{array}$ & Target Analvte & DF & $\begin{array}{c}\text { Result } \\
(\mathrm{ug} / \mathrm{kg})\end{array}$ & $\begin{array}{c}\text { IDAPA } \\
58.01 .24 \\
\text { Screening } \\
\text { Limit } \\
\text { (ug/kg) }\end{array}$ & $\begin{array}{c}\text { Region 9 } \\
\text { Industrial } \\
\text { Screening } \\
\text { Level } \\
\text { (ug/lgg) }\end{array}$ & $\begin{array}{l}\text { Critical } \\
\text { Pathway }\end{array}$ & $\begin{array}{c}\text { Exceed } \\
\text { Residential } \\
\text { Screening } \\
\text { Limit }\end{array}$ & $\begin{array}{c}\text { Exceed } \\
\text { Industrial } \\
\text { Screening } \\
\text { Limit }\end{array}$ & $\begin{array}{c}\text { Reporting } \\
\text { Limit } \\
\text { (ug/lg) }\end{array}$ & $\begin{array}{c}\text { Result } \\
\text { Qualifier }\end{array}$ \\
\hline$\Leftrightarrow$ & $91-20-3$ & NAPHTHALENE & 1 & 3.8 & $1.20 \mathrm{E}+02$ & & $\mathrm{VI}$ & no & & $3.80 \mathrm{E}+00$ & $\mathrm{U}$ \\
\hline 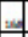 & $208-96-8$ & ACENAPHTHYLENE & 1 & 2.1 & $\mathrm{ND}$ & & & & & $3.80 \mathrm{E}+00$ & $\mathrm{~J}$ \\
\hline 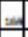 & $83-32-9$ & ACENAPHTHENE & 1 & 3.8 & $2.00 \mathrm{E}+05$ & & GWP & no & & $3.80 \mathrm{E}+00$ & $\mathrm{U}$ \\
\hline St & $86-73-7$ & FLUORENE & 1 & 3.8 & $2.40 \mathrm{E}+0 \mathrm{5}$ & & GWP & no & & $3.80 \mathrm{E}+00$ & $\mathrm{U}$ \\
\hline 政 & $85-01-8$ & PHENANTHRENE & 1 & 11 & $\mathrm{ND}$ & & & & & $3.80 \mathrm{E}+00$ & \\
\hline ats & $120-12-7$ & ANTHRACENE & 1 & 4.4 & $3.20 \mathrm{E}+06$ & & GWP & no & & $3.80 \mathrm{E}+00$ & \\
\hline tat & $206-44-0$ & FLUORANTHENE & 1 & 74 & $1.40 \mathrm{E}+06$ & & GWP & no & & $3.80 \mathrm{E}+00$ & \\
\hline ste & $129-00-0$ & PYRENE & 1 & 71 & $1.00 \mathrm{E}+05$ & & GWP & no & & $3.80 \mathrm{E}+00$ & \\
\hline at & $56-55-3$ & BENZO(A)ANTHRACENE & 1 & 44 & $9.00 \mathrm{E}+01$ & & GWP & no & & $3.80 \mathrm{E}+00$ & \\
\hline $\sin$ & $218-01-9$ & CHRYSENE & 1 & 40 & $9.50 \mathrm{E}+03$ & & GWP & no & & $3.80 \mathrm{E}+00$ & \\
\hline st & $205-99-2$ & BENZO(B)FLUOR_ANTHENE & 1 & 95 & $2.00 \mathrm{E}+02$ & & $\mathrm{DC}$ & no & & $3.80 \mathrm{E}+00$ & \\
\hline stat & $207-08-9$ & BENZO(K)FLUORANTHENE & 1 & 37 & $1.90 \mathrm{E}+03$ & & $\mathrm{DC}$ & no & & $3.80 \mathrm{E}+00$ & \\
\hline s & $50-32-8$ & BENZO(A)PYRENE & 1 & 58 & $2.00 \mathrm{E}+01$ & $3.90 \mathrm{E}+02$ & $\mathrm{DC}$ & YES & no & $3.80 \mathrm{E}+00$ & \\
\hline$\Rightarrow$ & $193-39-5$ & INDENO(1,2,3-CD)PYRENE & 1 & 29 & $\mathrm{ND}$ & & & & & $3.80 \mathrm{E}+00$ & \\
\hline 弯 & $53-70-3$ & DIBENZO $(\lambda, H), A N T H R A C E N E$ & 1 & 11 & ND & & & & & $3.80 \mathrm{E}+00$ & \\
\hline 皮 & $191-24-2$ & BENZO(G,H.I)PERYLENE & 1 & 34 & ND & & & & & $3.80 \mathrm{E}+00$ & \\
\hline-2 & $\begin{array}{c}\text { Sample } \\
\text { ID }\end{array}$ & Field ID: BEA031237_MFC & Lab I & [D: 12093 & $39-7$ & & & & & & \\
\hline 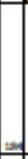 & $\begin{array}{c}\text { CAS } \\
\text { Number }\end{array}$ & Target Analvte & DF & $\begin{array}{c}\text { Result } \\
(\mathrm{ug} / \mathrm{kg})\end{array}$ & $\begin{array}{c}\text { IDAPA } \\
58.01 .24 \\
\text { Screening } \\
\text { Limit } \\
\text { (ug/kg) } \\
\end{array}$ & $\begin{array}{c}\text { Region } 9 \\
\text { Industrial } \\
\text { Screening } \\
\text { Level } \\
\text { (ug/kg) } \\
\end{array}$ & $\begin{array}{c}\text { Critical } \\
\text { Pathway } \\
\end{array}$ & $\begin{array}{c}\text { Exceed } \\
\text { Residential } \\
\text { Screening } \\
\text { Limit } \\
\end{array}$ & $\begin{array}{c}\text { Exceed } \\
\text { Industrial } \\
\text { Screening } \\
\text { Limit } \\
\end{array}$ & $\begin{array}{c}\text { Reporting } \\
\text { Limit } \\
\text { (ug/lkg) }\end{array}$ & $\begin{array}{c}\text { Result } \\
\text { Qualifier } \\
\end{array}$ \\
\hline 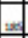 & $91-20-3$ & NAPHTHALENE & 1 & 3.8 & $1.20 \mathrm{E}+02$ & & $\mathrm{VI}$ & no & & $3.80 \mathrm{E}+00$ & $\mathrm{U}$ \\
\hline 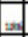 & $208-96-8$ & ACENAPHTHYLENE & 1 & 3.8 & $\mathrm{ND}$ & & & & & $3.80 \mathrm{E}+00$ & $\mathrm{U}$ \\
\hline st & $83-32-9$ & ACENAPHTHENE & 1 & 3.8 & $2.00 \mathrm{E}+05$ & & GWP & no & & $3.80 \mathrm{E}+00$ & $\mathrm{U}$ \\
\hline$\Rightarrow$ & $86-73-7$ & FLUORENE & 1 & 3.8 & $2.40 \mathrm{E}+05$ & & GWP & no & & $3.80 \mathrm{E}+00$ & $\mathrm{U}$ \\
\hline s & $85-01-8$ & PHENANTHRENE & 1 & 4.5 & $\mathrm{ND}$ & & & & & $3.80 \mathrm{E}+00$ & \\
\hline set & $120-12-7$ & ANTHRACENE & 1 & 1.5 & $3.20 \mathrm{E}+06$ & & GWP & no & & $3.80 \mathrm{E}+00$ & $\vec{J}$ \\
\hline sist & $206-44-0$ & FLUORANTHENE & 1 & 32 & $1.40 \mathrm{E}+06$ & & GTP & no & & $3.80 \mathrm{E}+00$ & \\
\hline 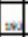 & $129-00-0$ & PYRENE & 1 & 25 & $1.00 \mathrm{E}+05$ & & GWP & no & & $3.80 \mathrm{E}+00$ & \\
\hline sis & $56-55-3$ & BENZO(A)ANTHRACENE & 1 & 20 & $9.00 \mathrm{E}+01$ & & GWP & no & & $3.80 \mathrm{E}+00$ & \\
\hline t & $218-01-9$ & CHRYSENE & 1 & 19 & $9.50 \mathrm{E}+03$ & & GWP & no & & $3.80 \mathrm{E}+00$ & \\
\hline 业 & $205-99-2$ & BENZO(B)FLUORANTHENE & 1 & 49 & $2.00 \mathrm{E}+02$ & & $\mathrm{DC}$ & no & & $3.80 \mathrm{E}+00$ & \\
\hline $\sin$ & $207-08-9$ & BENZO(K)FLUORANTHENE & 1 & 17 & $1.90 \mathrm{E}+03$ & & $\mathrm{DC}$ & no & & $3.80 \mathrm{E}+00$ & \\
\hline st & $50-32-8$ & BENZO(A)PYRENE & 1 & 27 & $2.00 \mathrm{E}+01$ & $3.90 \mathrm{E}+02$ & $\mathrm{DC}$ & YES & No & $3.80 \mathrm{E}+00$ & \\
\hline sef & $193-39-5$ & INDENO(1,2,3-CD)PYRENE & 1 & 9.4 & ND & & & & & $3.80 \mathrm{E}+00$ & \\
\hline st & $53-70-3$ & DIBENZO $(A, H), A N T H R A C E N E$ & 1 & 3.3 & $\mathrm{ND}$ & & & & & $3.80 \mathrm{E}+00$ & $\vec{J}$ \\
\hline 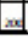 & $191-24-2$ & BENZO(G,H,I)PERYLENE & 1 & 10 & $\mathrm{ND}$ & & & & & $3.80 \mathrm{E}+00$ & \\
\hline
\end{tabular}




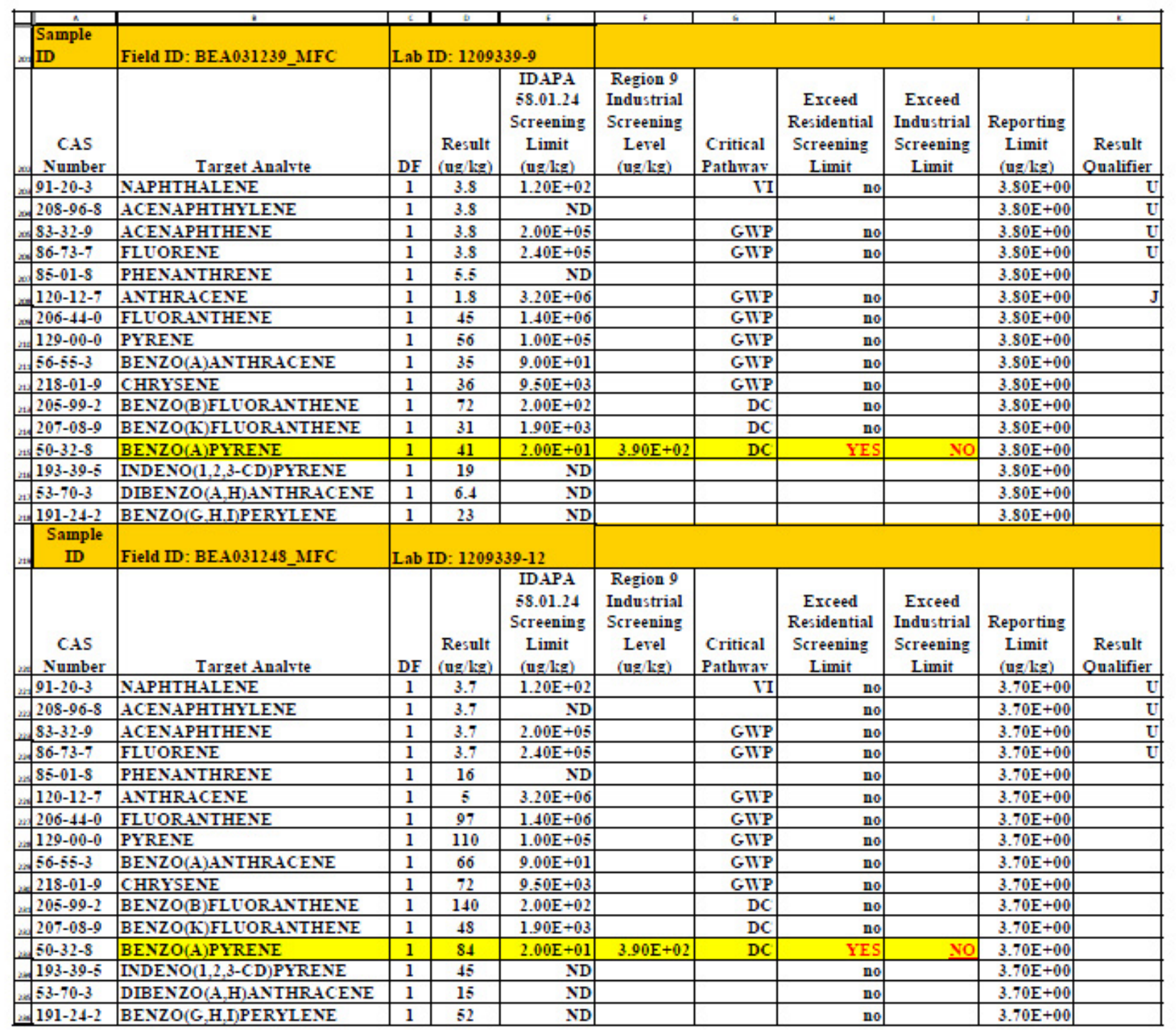




\begin{tabular}{|c|c|c|c|c|c|c|c|c|c|c|c|}
\hline \multirow{2}{*}{7} & \multirow{2}{*}{$\begin{array}{c}\text { Sample } \\
\text { ID }\end{array}$} & & \multicolumn{2}{|c|}{\begin{tabular}{|l|l|l|} 
& 0 \\
\end{tabular}} & \begin{tabular}{|l|} 
\\
\end{tabular} & \multicolumn{2}{|r|}{$\underline{5}$} & s & \multicolumn{2}{|r|}{1} & \multirow{2}{*}{ i } \\
\hline & & Field ID: BEA031250_MFC & \multicolumn{3}{|c|}{ Lab ID: $1209339-14$} & & & & & & \\
\hline ef & $\begin{array}{c}\text { CAS } \\
\text { Number }\end{array}$ & Target Analvte & DF & $\begin{array}{c}\text { Result } \\
(\mathrm{ug} / \mathrm{kg})\end{array}$ & $\begin{array}{c}\text { IDAPA } \\
58.01 .24 \\
\text { Screening } \\
\text { Limit } \\
\text { (ug/lgg) }\end{array}$ & $\begin{array}{c}\text { Region 9 } \\
\text { Industrial } \\
\text { Screening } \\
\text { Level } \\
\text { (ug/kg) }\end{array}$ & $\begin{array}{c}\text { Critical } \\
\text { Pathwav }\end{array}$ & $\begin{array}{c}\text { Exceed } \\
\text { Residential } \\
\text { Screening } \\
\text { Limit } \\
\end{array}$ & $\begin{array}{c}\text { Exceed } \\
\text { Industrial } \\
\text { Screening } \\
\text { Limit } \\
\end{array}$ & $\begin{array}{c}\text { Reporting } \\
\text { Limit } \\
(\mathrm{ug} / \mathrm{kg}) \\
\end{array}$ & $\begin{array}{c}\text { Result } \\
\text { Qualifier } \\
\end{array}$ \\
\hline 2 & $91-20-3$ & NAPHTHALENE & 1 & 3.4 & $1.20 \mathrm{E}+02$ & & $\mathrm{VI}$ & no & & $3.40 \mathrm{E}+00$ & $\mathrm{U}$ \\
\hline 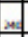 & $208-96-8$ & ACENAPHTHYLENE & 1 & 3.4 & $\mathbf{N D}$ & & & no & & $3.40 \mathrm{E}+00$ & $\mathrm{U}$ \\
\hline 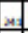 & $83-32-9$ & ACENAPHTHENE & 1 & 1.1 & $2.00 \mathrm{E}+0 \mathrm{~s}$ & & GIVP & no & & $3.40 \mathrm{E}+00$ & $\mathrm{~J}$ \\
\hline at & $86-73-7$ & FLUORENE & 1 & 3.4 & $2.40 \mathrm{E}+05$ & & GTP & no & & $3.40 \mathrm{E}+00$ & $\mathrm{U}$ \\
\hline 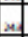 & $85-01-8$ & PHENANTHRENE & 1 & 4.6 & $\mathrm{ND}$ & & & no & & $3.40 \mathrm{E}+00$ & \\
\hline 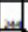 & $120-12-7$ & ANTHRACENE & 1 & 1.6 & $3.20 \mathrm{E}+06$ & & GWP & no & & $3.40 \mathrm{E}+00$ & $\mathrm{~J}$ \\
\hline 些 & $206-44-0$ & FLUORANTHENE & 1 & 15 & $1.40 \mathrm{E}+06$ & & GTP & no & & $3.40 \mathrm{E}+00$ & \\
\hline ant & $129-00-0$ & PYRENE & 1 & 13 & $1.00 \mathrm{E}+05$ & & GTV & no & & $3.40 \mathrm{E}+00$ & \\
\hline 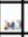 & $56-55-3$ & BENZO $(A) A N T H R A C E N E$ & 1 & 9.1 & $9.00 \mathrm{E}+01$ & & GWP & no & & $3.40 \mathrm{E}+00$ & \\
\hline 然 & $218-01-9$ & CHRYSENE & 1 & 7.4 & $9.50 \mathrm{E}+03$ & & GIVP & no & & $3.40 \mathrm{E}+00$ & \\
\hline ats & $205-99-2$ & BENZO(B)FLUORANTHENE & 1 & 19 & $2.00 \mathrm{E}+02$ & & $\mathrm{DC}$ & no & & $3.40 \mathrm{E}+00$ & \\
\hline 画 & $207-08-9$ & BENZO(K)FLUORANTHENE & 1 & 7.3 & $1.90 \mathrm{E}+03$ & & $\mathrm{DC}$ & no & & $3.40 \mathrm{E}+00$ & \\
\hline st & $50-32-8$ & BENZO $(A)$ PYRENE & 1 & 11 & $2.00 \mathrm{E}+01$ & $3.90 \mathrm{E}+02$ & DC & no & no & $3.40 \mathrm{E}+00$ & \\
\hline$\Delta$ & $193-39-5$ & INDENO(1,2,3-CD)PYRENE & 1 & 4.7 & $\mathbf{N D}$ & & & no & & $3.40 \mathrm{E}+00$ & \\
\hline st & $53-70-3$ & DIBENZO $(\lambda, H) A N T H R A C E N E$ & 1 & 1.5 & $\mathrm{ND}$ & & & no & & $3.40 \mathrm{E}+00$ & $\mathrm{~J}$ \\
\hline 至 & $191-24-2$ & BENZO(G.H.I)PERYLENE & 1 & 5.8 & $\mathrm{ND}$ & & & no & & $3.40 \mathrm{E}+00$ & \\
\hline$\Delta$ & $\begin{array}{c}\text { Sample } \\
\text { ID }\end{array}$ & Field ID: BEA031252_MFC & Lab I & [D: 12093 & $39-16$ & & & & & & \\
\hline sof & $\begin{array}{c}\text { CAS } \\
\text { Number }\end{array}$ & Iarget Analvte & DF & $\begin{array}{c}\text { Result } \\
(\mathrm{ug} / \mathrm{lg})\end{array}$ & \begin{tabular}{c|} 
IDAPA \\
58.01 .24 \\
Screening \\
Limit \\
(ug/lg) \\
\end{tabular} & $\begin{array}{c}\text { Region } 9 \\
\text { Industrial } \\
\text { Screening } \\
\text { Level } \\
\text { (ug/kg) }\end{array}$ & $\begin{array}{l}\text { Critical } \\
\text { Pathway }\end{array}$ & $\begin{array}{c}\text { Exceed } \\
\text { Residential } \\
\text { Screening } \\
\text { Limit } \\
\end{array}$ & $\begin{array}{c}\text { Exceed } \\
\text { Industrial } \\
\text { Screening } \\
\text { Limit } \\
\end{array}$ & $\begin{array}{c}\text { Reporting } \\
\text { Limit } \\
\text { (ug/kg) } \\
\end{array}$ & $\begin{array}{c}\text { Result } \\
\text { Qualifier } \\
\end{array}$ \\
\hline 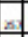 & $91-20-3$ & NAPHTHALENE & 7 & 24 & $1.20 \mathrm{E}+02$ & & $\mathrm{VI}$ & no & & $2.40 \mathrm{E}+01$ & $\mathrm{U}$ \\
\hline 植 & $208-96-8$ & ACENAPHTHYLENE & 7 & 24 & ND & & & no & & $2.40 \mathrm{E}+01$ & $\mathrm{U}$ \\
\hline st & $83-32-9$ & ACENAPHTHENE & 7 & 24 & $2.00 \mathrm{E}+05$ & & GWP & no & & $2.40 \mathrm{E}+01$ & $\mathrm{U}$ \\
\hline$a$ & $86-73-7$ & FLUORENE & 7 & 24 & $2.40 \mathrm{E}+05$ & & GIVP & no & & $2.40 \mathrm{E}+01$ & $\mathrm{U}$ \\
\hline$\Delta$ & $85-01-8$ & PHENANTHRENE & 7 & 29 & $\mathrm{ND}$ & & & no & & $2.40 \mathrm{E}+01$ & \\
\hline$a$ & $120-12-7$ & ANTHRACENE & 7 & 9.2 & $3.20 \mathrm{E}+06$ & & GIVP & no & & $2.40 \mathrm{E}+01$ & $\mathrm{~J}$ \\
\hline$g$ & $206-44-0$ & FLUORANTHENE & 7 & 160 & $1.40 \mathrm{E}+06$ & & GIVP & no & & $2.40 \mathrm{E}+01$ & \\
\hline$\Rightarrow$ & $129-00-0$ & PYRENE & 7 & 110 & $1.00 \mathrm{E}+05$ & & GTP & no & & $2.40 \mathrm{E}+01$ & \\
\hline 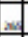 & $56-55-3$ & BENZO $(A)$ ANTHRACENE & 7 & 90 & $9.00 \mathrm{E}+01$ & & GTP & no & & $2.40 \mathrm{E}+01$ & \\
\hline 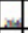 & $218-01-9$ & CHRYSENE & 7 & 88 & $9.50 \mathrm{E}+03$ & & GIVP & no & & $2.40 \mathrm{E}+01$ & \\
\hline 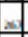 & $205-99-2$ & BENZO(B)FLUOR_ANTHENE & 7 & 150 & $2.00 \mathrm{E}+02$ & & $\mathrm{DC}$ & no & & $2.40 \mathrm{E}+01$ & \\
\hline$\Rightarrow$ & $207-08-9$ & BENZO(K)FLUORANTHENE & 7 & 62 & $1.90 \mathrm{E}+03$ & & DC & no & & $2.40 \mathrm{E}+01$ & \\
\hline s & $50-32-8$ & BENZO(A)PYRENE & 7 & 110 & $2.00 \mathrm{E}+01$ & $3.90 \mathrm{E}+02$ & $\mathrm{DC}$ & YES & No & $2.40 \mathrm{E}+01$ & \\
\hline 2 & $193-39-5$ & INDENO(1,2,3-CD)PYRENE & 7 & 70 & ND & & & no & & $2.40 \mathrm{E}+01$ & \\
\hline 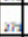 & $53-70-3$ & DIBENZO $(A, H) A N T H R A C E N E$ & 7 & 23 & $\mathrm{ND}$ & & & no & & $2.40 \mathrm{E}+01$ & $\mathrm{~J}$ \\
\hline at & $191-24-2$ & BENZO(G,H,I)PERYLENE & 7 & 77 & ND & & & no & & $2.40 \mathrm{E}+01$ & \\
\hline
\end{tabular}




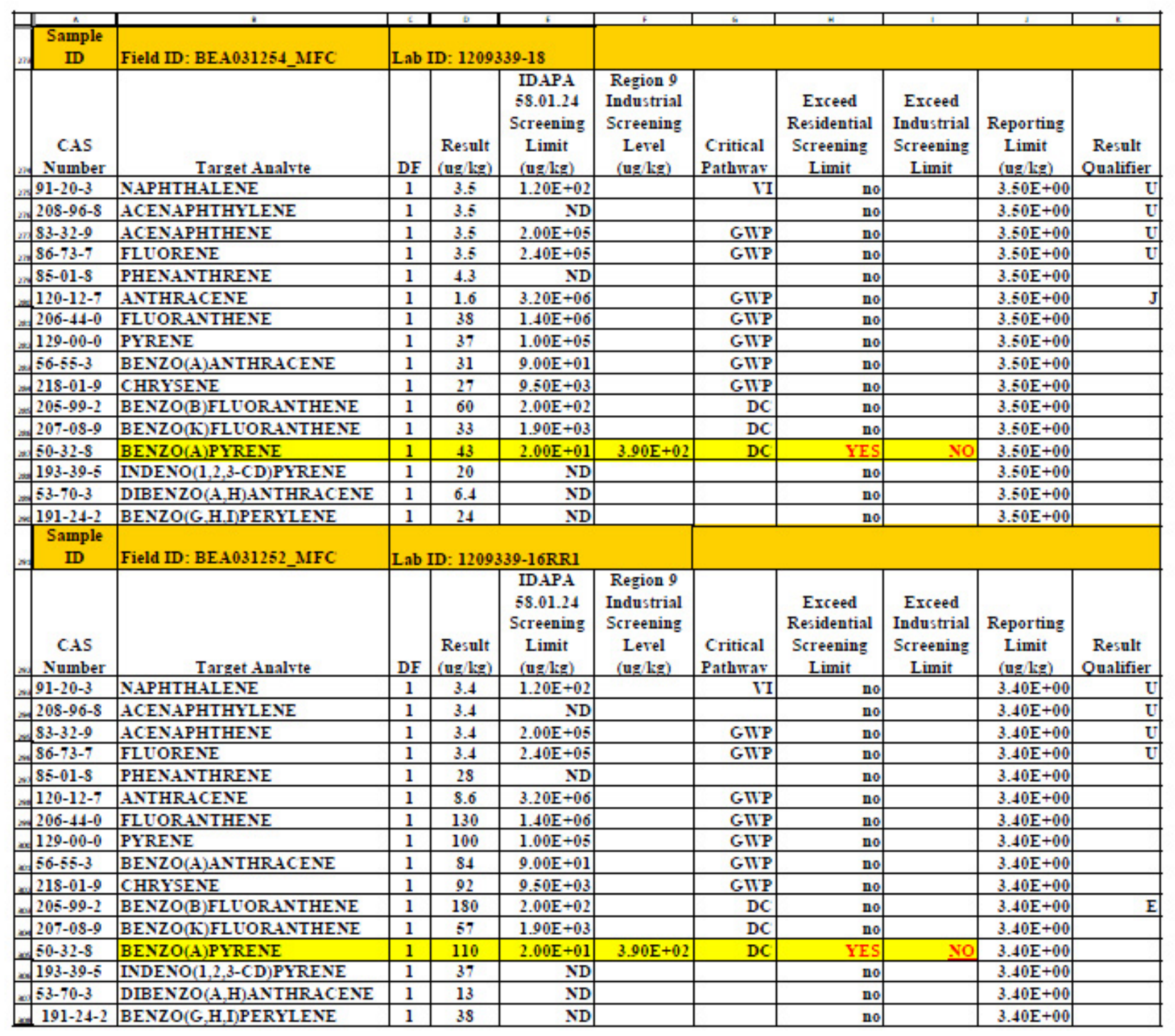




\section{Appendix J \\ E-mail to DEQ with Soil Analytical Analysis \#1 and \#2 and Spreadsheet Comparison of EPA Region 9 Industrial Screening Levels vs. IDAPA Residential Screening Levels}

MFC Biodiesel Tank Samples and Screening Levels - kerry.nisson(@)inl.gov - Idaho National Laboratory ... Page 1 or 1

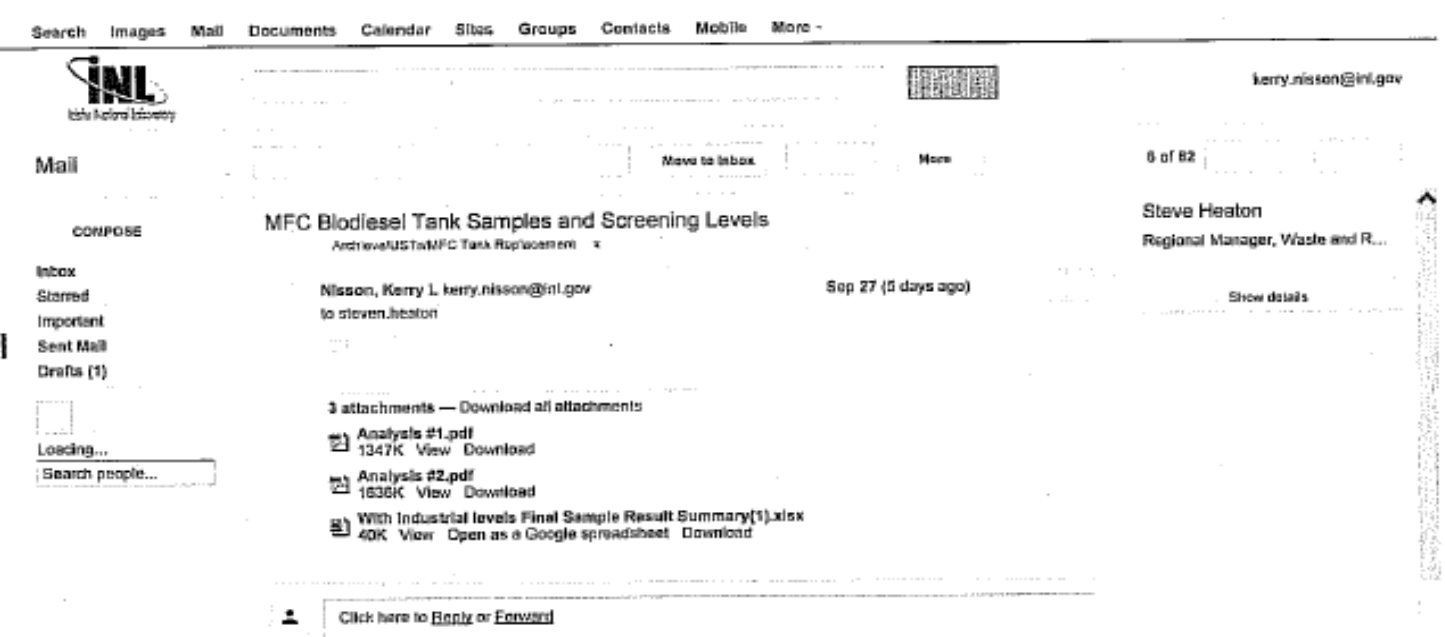

